Merci d'utiliser le titre suivant lorsque vous citez ce document :

Jones, D. (2010-08-01), « Analyse de la composition du soutien aux producteurs : Nouveaux outils et méthodes », Éditions OCDE, Paris.

http://dx.doi.org/10.1787/5km91ndn3tkc-fr

\title{
Analyse de la composition du soutien aux producteurs
}

\author{
NOUVEAUX OUTILS ET MÉTHODES
}

Darryl Jones

La version originale de ce document a été publiée comme suit :

Jones, D. (2010-08-01), "Analysing the Composition of Producer Support: New Tools and Methods", OECD Food, Agriculture and Fisheries Papers, No. 32, OECD Publishing, Paris.

http://dx.doi.org/10.1787/5km91ndxhsnx-en 


\title{
ANALYSE DE LA COMPOSITION DU SOUTIEN AUX PRODUCTEURS :
} NOUVEAUX OUTILS ET MÉTHODES

\author{
Darryl Jones*
}

* Consultant 


\section{Résumé}

Ce rapport examine la capacité d'analyse du système de classification des mesures de politique agricole entrant dans l'Estimation du Soutien aux Producteurs (ESP) de l'OCDE en termes de suivi de l'évolution des politiques. Ce système de catégories, sous-catégories et étiquettes est fondé sur la forme sous laquelle les transferts sont accordés aux agriculteurs. L'analyse des données correspondant aux catégories, souscatégories et étiquettes de la classification des mesures dans l'ESP permet un examen critique des forces et des faiblesses de ce système. Il permet d'obtenir une image utile de certaines des différences importantes dans la mise en œuvre des politiques de soutien à l'agriculture au cours du temps et entre pays, mais il est bien évidemment limité par les catégories et labels prédéterminés. Cependant, la base de données contient aussi une abondance d'informations sur chaque mesure gouvernementale, sur lesquelles une analyse plus détaillée peut s'appuyer.

Mots clés : politique agricole, soutien à l'agriculture, soutien aux producteurs, ESP, composition du soutien, suivi et évaluation des politiques. 


\section{Table des matières}

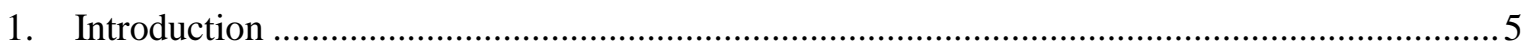

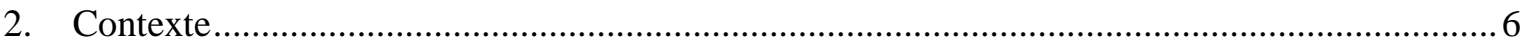

3. Possibilités analytiques offertes par l'ESP — aspects théoriques ............................................ 10

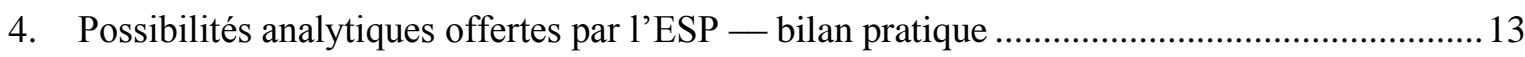

5. Le potentiel analytique de l'ESP — Autres considérations pratiques ......................................17

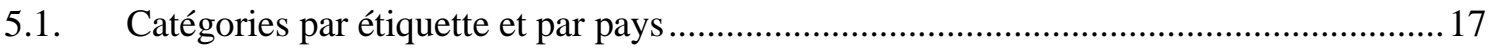

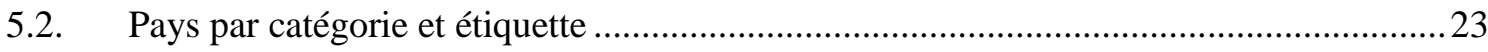

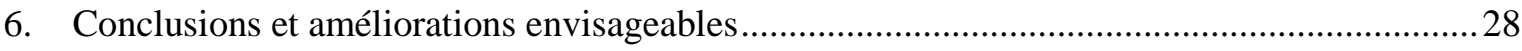

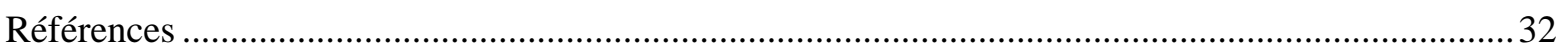

Annexe 1. Intitulés et définitions des catégories et étiquettes des mesures de l'ESP.........................33

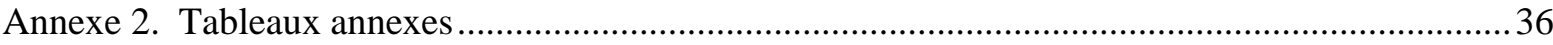





\title{
Analyse de la composition du soutien aux producteurs : nouveaux outils et méthodes
}

\begin{abstract}
À la suite d'une période de consultation entre le Secrétariat de l'OCDE, les pays membres et des experts, un système révisé de classification des catégories, souscatégories et étiquettes relatives à l'estimation du soutien aux producteurs (ESP) a été mis en place en 2007. Le besoin s'était fait sentir de revisiter le système de classification en raison de l'évolution permanente de la politique agricole des pays de l'OCDE. Ce qui change, ce n'est pas la méthode de calcul du niveau du soutien aux producteurs, mais le mode d'utilisation de la composition de ce soutien — c'est-à-dire la forme sous laquelle les transferts sont accordés aux agriculteurs - pour suivre et évaluer l'évolution de l'action publique. En effet, les changements apportés à la composition du soutien aux producteurs sont progressivement devenus un élément essentiel de l'évaluation de la réforme des politiques agricoles.
\end{abstract}

Grâce à l'augmentation du nombre des catégories permettant de classer les mesures gouvernementales et à l'introduction six étiquettes pour qualifier chacune de ces mesures, le système révisé de classification des mesures de l'ESP a renforcé l'intérêt des indicateurs de soutien établis par l'OCDE pour suivre et évaluer la composition du soutien. Ce faisant, la complexité de la base de données s'en est trouvé grandement accrue.

L'objectif de ce rapport est d'étudier les possibilités analytiques offertes par le système révisé de catégories, sous-catégories et étiquettes pour suivre l'évolution de l'action publique, examinant de manière critique les forces et les faiblesses du système de classification en vigueur.

\section{Introduction}

L'objet de ce rapport est l'examen des potentialités analytiques de l'actuel système de classification des mesures entrant dans l'ESP, qui a été introduit en 2007 pour suivre et évaluer l'évolution des politiques agricoles. Deux questions importantes se posent: les étiquettes les plus utiles sont-elles présentées de manière adéquate dans les rapports annuels (Politiques agricoles des pays de l'OCDE : Suivi et évaluation ou Panorama) ? De nouveaux perfectionnements sont-ils susceptibles d'améliorer la puissance analytique de la base de données?

Ce rapport explique tout d'abord l'évolution du système de classification des mesures de l'ESP et définit la terminologie retenue pour l'analyse. Elle aborde ensuite, d'un point de vue théorique, ce que les différentes catégories et étiquettes peuvent révéler au sujet des mesures gouvernementales, notamment par rapport à d'importants éléments de la réforme des politiques convenus par les ministres de l'Agriculture des pays de l'OCDE, en l'occurrence le découplage, le ciblage et l'adaptation. Sont ensuite brièvement relatées trois actions qui ont été entreprises dans l'optique d'utiliser le système de classification révisé pour suivre et évaluer l'évolution de l'action publique 
depuis son introduction : l'édition 2009 du rapport annuel Politiques agricoles des pays de l'OCDE : Suivi et évaluation (OCDE, 2009a), les mesures liées aux risques (OCDE, 2009 b) et les paiements agroenvironnementaux (chapitre 2 de OCDE, 2009a). La section suivante est consacrée à l'analyse des informations contenues dans la base de données des ESP et ESC, par pays, catégorie et étiquette. Cette analyse vise à montrer dans quelle mesure les informations contenues dans la base de données des ESP et des ESC mises à la disposition du public peuvent être utilisées par tout un chacun. La dernière section porte quant à elle sur l'utilité effective du système de classification comme outil analytique. L'annexe 1 donne les intitulés et les définitions des catégories et étiquettes, tandis que l'annexe 2 contient des tableaux détaillés sur la composition de l'ESP.

\section{Contexte ${ }^{1}$}

Depuis que l'OCDE a commencé à suivre et évaluer le soutien accordé au secteur agricole, au milieu des années 80 , elle a toujours fait appel à un système de classification des mesures dans différentes catégories de soutien afin de pouvoir évaluer l'évolution de l'action publique par rapport aux critères définis par les ministres des pays de l'OCDE pour la réforme des politiques. Il y avait à l'origine cinq catégories de mesures de l'ESP, la classification étant faite en fonction du type de soutien : Soutien des prix du marché (MPS), Paiements directs, Réduction du coût des intrants, Services d'intérêt général et Autres. À cette époque, la vaste majorité du soutien accordé aux producteurs relevait de mesures de soutien des prix du marché (par exemple : contingents d'importation, droits de douane élevés et subventions à l'exportation) et de paiements compensatoires liés directement à la production de produits particuliers. Depuis lors, les mesures de soutien à l'agriculture mises en place dans la zone de l'OCDE se sont multipliées et ont gagné en complexité.

Ces évolutions traduisent, entre autres, le mouvement de «découplage » de l'octroi d'un soutien en fonction de la production de tel ou tel produit et de son « recouplage » en fonction d'autres critères. Les mesures mises en œuvre prévoient de plus en plus des transferts, non pas sur la base de la production, mais sur d'autres éléments, comme la superficie cultivée, le revenu agricole total ou des critères non liés à des produits de base. Nombre d'entre elles accordent désormais un soutien en fonction d'une combinaison de «prix et production courants et antérieurs », souvent assortie de contraintes sur les conditions d'éligibilité aux paiements. Il ressort en outre de l'analyse effectuée par l'OCDE sur les effets des mesures sur la production, les échanges, le revenu et l'environnement, que ce n'est pas seulement le niveau du soutien, mais le mode de mise en œuvre de la mesure en question et des conditions auxquelles est subordonnée le versement du soutien, qui déterminent l'impact des mesures sur ces facteurs, et que ces effets peuvent varier considérablement d'une mesure à l'autre. Ces évolutions ont conduit à porter une attention plus grande à la composition du soutien dans le cadre du processus de suivi et d'évaluation de la réforme des politiques agricoles.

Deux modifications majeures ont été apportées en 1999 au système de classification. En premier lieu, les mesures apportant collectivement un soutien aux producteurs par le biais de « services d'intérêt général » à l'ensemble du secteur agricole ont été séparées de l'ESP et prises en compte dans un indicateur propre, à savoir l'estimation du soutien aux

1. On trouvera dans le "Manuel de calcul de l'ESP" (OCDE, 2008a) une explication plus complète du système de classification des mesures de l'ESP ainsi que son historique. 
services d'intérêt général (ESSG). En conséquence, l'ESP comprend seulement la valeur des transferts découlant des mesures octroyant un soutien aux producteurs à titre individuel. En second lieu, la classification des mesures au sein de l'ESP a été répartie entre sept catégories reflétant leurs critères de mise en œuvre, c'est-à-dire les conditions au titre desquelles les transferts associés sont versés aux agriculteurs, ou les conditions d'éligibilité à ce paiement: MPS, Paiements au titre de la production, Paiements au titre des superficies ou du nombre d'animaux, Paiements au titre des droits antérieurs, Paiements au titre de l'utilisation d'intrants, Paiements reposant sur les contraintes sur les intrants et Paiements divers.

Ces nouveautés représentaient certes une amélioration significative des possibilités d'analyse de la composition du soutien, mais l'évolution permanente de la conception de mesures agricoles souvent complexes rendait de plus en plus difficile la classification des nouvelles mesures dans les catégories existantes. C'est la raison pour laquelle, en 2007, un système de classification révisé a été adopté, lequel se caractérisait par deux grands changements : la révision des catégories et l'introduction d'étiquettes.

On a tout d'abord procédé à la révision et à l'extension des catégories, même si celles-ci continuaient de reposer sur des critères de mise en œuvre. On compte désormais 14 catégories de mesures de l'ESP au niveau détaillé (ou sous-catégories), qui se regroupent en sept catégories principales. Ces catégories figurent dans le tableau 2.1 sous forme de lignes, leur définition complète étant donnée dans l'annexe 1.

Il convient de noter que l'on peut se référer à ces catégories soit sous forme alphanumérique (A2, C, F3), soit par un système d'abréviation (mnémonique) tel qu'utilisé dans la base de données des ESP et des ESC, par exemple la catégorie «Paiements au titre de la production » peut être désignée soit par la catégorie A2, soit par l'abréviation PO. Toutes les données enregistrées depuis 1986 sont désormais classées selon ce système.

Les critères de classification d'une mesure dans telle ou telle catégorie sont exprimés par une séquence standard de questions (arbre de décision). Pour que la classification soit exacte et cohérente, ces questions sont posées pour chaque mesure. Même si une mesure peut dépendre de plusieurs de ces critères, on la classe d'après le premier critère applicable. On trouvera dans les documents « Définitions et sources » consultables sur le site web pour chaque pays, des informations complètes sur le mode de classification de chacune des mesures en vigueur dans les différents pays.

L'autre modification majeure apportée au système de classification a été l'introduction d'étiquettes. Lorsqu'une mesure est classée dans une catégorie particulière, elle est également qualifiée par plusieurs étiquettes apportant des détails supplémentaires sur les critères de mise en ouvre. Les six groupes d'étiquettes apparaissent sous la forme de colonnes dans le tableau 2.1, leur définition complète étant donnée dans l'annexe 1. Trois de ces groupes offrent un choix entre deux étiquettes (par exemple : les mesures se voient attribuer une étiquette soit Taux de paiement «fixe», soit Taux de paiement «variable»), deux groupes offrent trois options, et un groupe offre quatre options. Les options offertes par chaque groupe d'étiquettes s'excluent mutuellement, de sorte qu'il n'est possible d'attribuer à une mesure qu'une seule des options disponibles pour chaque étiquette. 
Tableau 2.1. Catégories et étiquettes définies pour les mesures de l'ESP

\begin{tabular}{|c|c|c|c|c|c|c|c|}
\hline \multirow{3}{*}{$\begin{array}{c}\text { CATÉGORIES, } \\
\text { principales }\end{array}$} & \multirow{3}{*}{$\begin{array}{l}\text { CATÉGORIES } \\
\text { Détaillées ou } \\
\text { sous-catégories }\end{array}$} & \multicolumn{6}{|c|}{ ÉTIQUETTES } \\
\hline & & $\begin{array}{c}\text { Limitation } \\
\text { de la } \\
\text { production } \\
\text { courante } \\
\text { de produits } \\
\text { de base et } \\
\text { des } \\
\text { paiements }\end{array}$ & $\begin{array}{c}\text { Taux de } \\
\text { paiement }\end{array}$ & $\begin{array}{l}\text { Contraintes sur } \\
\text { les intrants }\end{array}$ & Éligibilité fondée sur 2 & $\begin{array}{l}\text { Éligibilité } \\
\text { fondée sur } 2\end{array}$ & $\begin{array}{l}\text { Exceptions } \\
\text { concernant } \\
\text { les produits }\end{array}$ \\
\hline & & $\begin{array}{l}\text { Limitation/ } \\
\text { Pas de } \\
\text { limitation } \\
\end{array}$ & $\begin{array}{c}\text { Fixes/ } \\
\text { Variables }\end{array}$ & $\begin{array}{c}\text { Avec } \\
\text { (obligatoires/Avec } \\
\text { (volontaires)Sans }\end{array}$ & $\begin{array}{l}\text { Produit(s) de base : Un } \\
\text { seul /un groupe / tous }\end{array}$ & $\begin{array}{l}\text { Superficies/nom } \\
\text { bre d'animaux/ } \\
\text { recettes/revenus }\end{array}$ & Avec/sans \\
\hline $\begin{array}{l}\text { A. Soutien au titre de la } \\
\text { production de produits } \\
\text { de base }(\mathrm{CO})\end{array}$ & $\begin{array}{l}\text { A1. Soutien des prix du marché } \\
\text { (MPS) } \\
\text { A2. Paiements au titre de la } \\
\text { production (PO) }\end{array}$ & $\begin{array}{l}\mathrm{Y} / \mathrm{N} \\
\mathrm{Y} / \mathrm{N}\end{array}$ & $\begin{array}{l}F / V \\
F / V\end{array}$ & $\begin{array}{c}\text { n.a. } \\
\text { M/V/No }\end{array}$ & $\begin{array}{l}\mathrm{SC} / \mathrm{GC} / \mathrm{AC} \\
\mathrm{SC} / \mathrm{GC} / \mathrm{AC}\end{array}$ & $\begin{array}{l}\text { n.a. } \\
\text { n.a. }\end{array}$ & $\begin{array}{l}\text { n.a. } \\
\text { n.a. }\end{array}$ \\
\hline $\begin{array}{l}\text { B. Paiements au titre de } \\
\text { l'utilisation d'intrants } \\
\text { (PI) }\end{array}$ & $\begin{array}{l}\text { B1. Utilisation d'intrants variables } \\
\text { (PIV) } \\
\text { B2. Formation de capital fixe (PIF) } \\
\text { B3. Services utilisés sur } \\
\text { l'exploitation (PIS) }\end{array}$ & $\begin{array}{l}\mathrm{Y} / \mathrm{N} \\
\mathrm{Y} / \mathrm{N} \\
\mathrm{Y} / \mathrm{N}\end{array}$ & $\begin{array}{l}F / V \\
F / V \\
F / V\end{array}$ & $\begin{array}{l}\mathrm{M} / \mathrm{V} / \mathrm{No} \\
\mathrm{M} / \mathrm{V} / \mathrm{No} \\
\mathrm{M} / \mathrm{V} / \mathrm{No}\end{array}$ & $\begin{array}{l}\mathrm{SC} / \mathrm{GC} / \mathrm{AC} \\
\mathrm{SC} / \mathrm{GC} / \mathrm{AC} \\
\mathrm{SC} / \mathrm{GC} / \mathrm{AC}\end{array}$ & $\begin{array}{l}\text { n.a. } \\
\text { n.a. } \\
\text { n.a. }\end{array}$ & $\begin{array}{l}\text { n.a. } \\
\text { n.a. } \\
\text { n.a. }\end{array}$ \\
\hline $\begin{array}{l}\text { C. Paiements au titre } \\
\text { des } \mathrm{S} / \mathrm{Na} / \mathrm{Rec} / \mathrm{Rev} \\
\text { courants, production } \\
\text { requise (PC) }\end{array}$ & $\begin{array}{l}\text { C1. Au titre des recettes/revenus } \\
\text { courants } \\
\text { C2. Au titre des superficies } \\
\text { /nombres d'animaux courants }\end{array}$ & $\begin{array}{l}\mathrm{Y} / \mathrm{N} \\
\mathrm{Y} / \mathrm{N}\end{array}$ & $\begin{array}{l}F / V \\
F / V\end{array}$ & $\begin{array}{l}\mathrm{M} / \mathrm{V} / \mathrm{No} \\
\mathrm{M} / \mathrm{V} / \mathrm{No}\end{array}$ & $\begin{array}{l}\mathrm{SC} / \mathrm{GC} / \mathrm{AC} \\
\mathrm{SC} / \mathrm{GC} / \mathrm{AC}\end{array}$ & $\begin{array}{l}\mathrm{R} / \mathrm{I} \\
\mathrm{A} / \mathrm{An}\end{array}$ & $\begin{array}{l}\text { n.a. } \\
\text { n.a. }\end{array}$ \\
\hline $\begin{array}{l}\text { D. Paiements au titre } \\
\text { des } \mathrm{S} / \mathrm{Na} / \mathrm{Rec} / \mathrm{Rev} \text { non } \\
\text { courants, production } \\
\text { requise (PHR) } \\
\end{array}$ & $\begin{array}{l}\text { D. Paiements au titre des } \\
\mathrm{S} / \mathrm{Na} / \mathrm{Rec} / \mathrm{Rev} \text { non courants, } \\
\text { production requise }\end{array}$ & $\mathrm{Y} / \mathrm{N}$ & $\mathrm{F} / \mathrm{V}$ & $\mathrm{M} / \mathrm{V} / \mathrm{No}$ & $\mathrm{SC} / \mathrm{GC} / \mathrm{AC}$ & $\mathrm{A} / \mathrm{An} / \mathrm{R} / \mathrm{I}$ & n.a. \\
\hline $\begin{array}{l}\text { E. Paiements au titre } \\
\text { des S/Na/Rec/Rev non } \\
\text { courants, production } \\
\text { facultative (PHNR) }\end{array}$ & $\begin{array}{l}\text { E1. Taux variables } \\
\text { E2. Taux fixes }\end{array}$ & $\begin{array}{l}\mathrm{Y} / \mathrm{N} \\
\mathrm{Y} / \mathrm{N}\end{array}$ & $\mathrm{V}$ & $\begin{array}{l}\mathrm{M} / \mathrm{V} / \mathrm{No} \\
\mathrm{M} / \mathrm{V} / \mathrm{No}\end{array}$ & n.a. & $\begin{array}{l}\mathrm{A} / \mathrm{An} / \mathrm{R} / \mathrm{I} \\
\mathrm{A} / \mathrm{An} / \mathrm{R} / \mathrm{I}\end{array}$ & $\mathrm{Y} / \mathrm{N}$ \\
\hline
\end{tabular}




\begin{tabular}{|c|c|c|c|c|c|c|c|}
\hline \multirow{3}{*}{$\begin{array}{l}\text { CATÉGORIES, } \\
\text { principales }\end{array}$} & \multirow{3}{*}{$\begin{array}{c}\text { CATÉGORIES } \\
\text { Détaillées ou sous-catégories }\end{array}$} & \multicolumn{6}{|c|}{ ÉTIQUETTES } \\
\hline & & $\begin{array}{l}\text { Limitation } \\
\text { de la } \\
\text { production } \\
\text { courante } \\
\text { de produits } \\
\text { de base et } \\
\text { des } \\
\text { paiements } \\
\end{array}$ & $\begin{array}{l}\text { Taux de } \\
\text { paiement }\end{array}$ & $\begin{array}{l}\text { Contraintes sur } \\
\text { les intrants }\end{array}$ & $\begin{array}{l}\text { Éligibilité fondée } \\
\text { sur } 2\end{array}$ & $\begin{array}{c}\text { Éligibilité } \\
\text { fondée sur } 2\end{array}$ & $\begin{array}{l}\text { Exceptions } \\
\text { concernant } \\
\text { les produits }\end{array}$ \\
\hline & & $\begin{array}{l}\text { Limitation/ } \\
\text { Pas de } \\
\text { limitation }\end{array}$ & $\begin{array}{l}\text { Fixes/ } \\
\text { Variables }\end{array}$ & $\begin{array}{c}\text { Avec } \\
\text { (obligatoires/Avec } \\
\text { (volontaires)/Sans }\end{array}$ & $\begin{array}{l}\text { Produit(s) de base : } \\
\text { Un seul /un groupe / tous }\end{array}$ & $\begin{array}{l}\text { Superficies/nom } \\
\text { bre d'animaux/ } \\
\text { recettes/revenus }\end{array}$ & Avec/sans \\
\hline $\begin{array}{l}\text { F. Paiements selon des } \\
\text { critères non liés à des } \\
\text { produits de base (PN) }\end{array}$ & $\begin{array}{l}\text { F1. Retrait de ressources à long } \\
\text { terme (PNLT) } \\
\text { F2. Production de produits } \\
\text { particuliers autres que des produits } \\
\text { de base (PNSO) } \\
\text { F3. Autres critères non liés à des } \\
\text { produits de base (PNOP) }\end{array}$ & $\begin{array}{l}\mathrm{Y} / \mathrm{N} \\
\mathrm{Y} / \mathrm{N} \\
\mathrm{Y} / \mathrm{N}\end{array}$ & $\begin{array}{l}\text { n.a. } \\
\text { n.a. }\end{array}$ & $\begin{array}{l}\text { V } \\
\text { n.a. }\end{array}$ & $\begin{array}{l}\text { n.a. } \\
\text { n.a. } \\
\text { n.a. }\end{array}$ & $\begin{array}{l}\text { n.a. } \\
\text { n.a. } \\
\text { n.a. }\end{array}$ & $\begin{array}{l}\text { n.a. } \\
\text { n.a. }\end{array}$ \\
\hline $\begin{array}{l}\text { G. Paiements divers } \\
\text { (PM) }\end{array}$ & G. Paiements divers (PM) & n.a. & n.a. & n.a. & n.a. & n.a. & n.a. \\
\hline
\end{tabular}

Y/N : Oui ou non; F/V : fixes ou variables; M/V/No : contraintes obligatoires/contraintes volontaires/sans contraintes; A/An/R/I : superficie cultivée/nombre d'animaux/recettes/revenu ; "n.a. » : étiquettes non applicables aux mesures d'une catégorie donnée ; «SC » et « Avec (volontaires) » : ces étiquettes s'appliquent par définition aux mesures d'une catégorie donnée.

1. Les définitions des catégories et étiquettes figurent dans l'annexe 1.

2. Les abréviations utilisées pour les catégories (entre parenthèses) sont celles qui sont employées dans la base de données des ESP et des ESC. 
Les étiquettes offrent ainsi la possibilité d'analyser la composition du soutien aux producteurs selon différents points de vue, de procéder au regroupement de transferts mettant spécifiquement en exergue un critère de mise en œuvre utilisé dans les mesures adoptées. Néanmoins, toutes les étiquettes ne peuvent être appliquées à l'ensemble des catégories de mesures de l'ESP. Ainsi, comme on le voit dans le tableau 2.1, on ne peut accoler aux mesures relevant des catégories $\mathrm{E}, \mathrm{F}$ et $\mathrm{G}$ l'étiquette indiquant si un paiement est versé reposant sur un seul produit, d'un groupe de produits ou de tous les produits en général. Dès lors que l'on fait appel à des étiquettes pour analyser la composition du soutien, il faut donc être attentif à l'intérêt que présentent les données pour l'ESP.

On peut, le cas échéant, se servir des étiquettes comme sous-catégorie de telle ou telle catégorie dans le but de rendre compte des priorités ou des centres d'intérêts prévalant à un moment donné. Cette opération peut se faire de manière «formelle» lors de la construction des fichiers par pays ou de manière informelle lors de la présentation des résultats. À titre illustratif, deux exemples peuvent être donnés. Tout d'abord, on utilise actuellement l'étiquette indiquant si le taux de paiement est variable ou fixe pour créer des sous-catégories «formelles» pour la catégorie E. On a par ailleurs recours à l'étiquette relative aux contraintes sur les intrants pour la présentation de sous-catégories des catégories B1, B2 et B3 dans l'édition 2009 du rapport sur les Politiques agricoles des pays de l'OCDE. Rien n'empêche d'appliquer des sous-catégories analogues pour ces deux étiquettes ou toute autre étiquette à d'autres catégories de mesures de l'ESP auxquelles ces étiquettes sont applicables.

\section{Possibilités analytiques offertes par l'ESP — aspects théoriques}

Du point de vue analytique, le système de classification en catégories permet de suivre l'évolution de l'action publique en déterminant :

- l'élément au titre duquel le transfert est accordé : production (catégorie A), intrants (catégorie B), superficies/nombre d'animaux/recettes/revenus (catégories C, D et E), critères non liés à des produits de base (catégorie F) ;

- si le soutien est accordé au titre d'un élément courant (catégories A, B, C, F) ou non courant (historique ou fixe) (catégories D et E) ;

- si la production est requise (catégories $\mathrm{C}$ et $\mathrm{D}$ ) ou facultative (catégorie $\mathrm{E}$ ).

Deux des catégories (tableau 2.1) étendent les possibilités analytiques des catégories en apportant des informations supplémentaires directement liées aux critères de mise en œuvre (c'est-à-dire les conditions régissant l'octroi des transferts aux producteurs) pris en compte dans ces catégories.

- L'étiquette qualifiant l'éligibilité aux paiements au titre des $\mathrm{S} / \mathrm{Na} / \mathrm{Rec} / \mathrm{Rev}$ apporte de nouvelles informations sur l'élément au titre duquel le transfert est opéré en détaillant les quatre éléments de soutien pour chaque mesure figurant dans les catégories $\mathrm{C}, \mathrm{D}$ et E. Compte tenu de la classification en sous-catégories, cette étiquette prévoit sept options pour répertorier les transferts.

- L'étiquette qualifiant l'éligibilité aux paiements fondée sur la subordination à des produits de base apporte des informations complémentaires sur le type de production de produits de base requise dans les catégories $\mathrm{A}, \mathrm{B}, \mathrm{C}$ et $\mathrm{D}$, et indique si l'octroi du paiement repose sur la production d'un seul produit (SC), d'un groupe de produits (GC) ou de tous les produits $(\mathrm{AC})$. Dès lors que la mesure considérée requiert la production 
d'un seul produit ou d'un groupe de produits, les produits sont mentionnés de manière explicite.

Une des étiquettes apporte des informations qui viennent compléter les autres critères de mise en œuvre utilisés pour définir les catégories.

- L'étiquette indiquant si le taux de paiement prévu par une mesure est fixe ou variable précise les informations données par les catégories quant au versement du soutien sur une base courante ou non courante. Si ce taux (soutien par unité de production, en fonction des intrants, des superficies, du nombre d'animaux, etc.) est explicitement lié aux variations des prix courants perçus ou payés par les agriculteurs, ou à la production/rendement courant atteint, ou encore au revenu courant obtenu, alors la mesure en question se voit accoler l'étiquette « variable », c'est-à-dire qu'elle est liée aux conditions courantes du marché. Dans le cas contraire, elle se voit accoler l'étiquette "fixe» en ce sens que le taux de paiement est déterminé par d'autres facteurs, ce qui ne signifie toutefois pas que le taux prévu par une mesure étiquetée «fixe» a changé au fil des ans. Il peut en effet évoluer ${ }^{2}$, mais il ne varie pas directement d'une année sur l'autre en fonction de les conditions courantes de l'exploitation. Cette étiquette s'applique aux mesures classées dans les catégories A à E.

Les trois autres étiquettes apportent des informations supplémentaires sur les critères de mise en œuvre qui diffèrent de celles utilisées pour les catégories. Du point de vue analytique, ces étiquettes permettent de déterminer :

- Si le soutien impose une limitation soit sur la production de produits de base bénéficiant du transfert (par le biais de quotas de production ou d'exigences de mise hors production de terres, par exemple), soit sur la valeur des transferts dont les agriculteurs peuvent bénéficier dans le cadre de la mesure considérée (par exemple, par plafonnement des superficies ou du nombre d'animaux servant de base pour la détermination du paiement). Cette étiquette s'applique aux mesures classées dans les catégories A à F.

- Si le soutien impose des contraintes sur l'utilisation d'intrants, des pratiques de production particulières ou des divers critères environnementaux ou sociétaux (par exemple, en lien avec le bien-être des animaux). Lorsque ces contraintes, pratiques ou critères sont considérées comme une exigence fondamentale des bonnes pratiques agricoles ou renvoient à une réglementation, les mesures en question se voient accoler l'étiquette «avec (contraintes) obligatoires ». En revanche, lorsque ces contraintes, pratiques ou critères vont au-delà d'une exigence de base ou de réglementations à caractère général (par exemple: implantation de bandes tampon, méthodes de production extensives ou agriculture biologique), elles se voient accoler l'étiquette « avec (contraintes) volontaires ». Cette dernière signifie que les agriculteurs sont libres de choisir de participer au programme pour bénéficier du transfert, mais qu'une fois engagés dans ce programme, ils sont tenus de respecter les conditions définies. En d'autres termes, cette étiquette indique que la mesure visée impose aux agriculteurs de respecter des contraintes, pratiques ou critères plus rigoureux que l'exigence de base et que, pour bénéficier du paiement, ils acceptent une contractualisation à titre volontaire. Cette étiquette s'applique aux mesures classées dans les catégories A2 à E.

2. Rien n'empêche non plus une mesure prévoyant le versement de paiements sur une base historique/fixe d'être ultérieurement fondée sur une autre base. 
- Si le soutien interdit la production de certains produits de base comme critère d'éligibilité au paiement. Une mesure à laquelle est accolée l'étiquette «avec exceptions » indique que l'agriculteur ne sera éligible à un paiement que s'il ne produit pas certains produits, ce qui limite ses choix de production. Cette étiquette indique que les conditions définies par la mesure en question créent une contrainte sur certaines décisions de production. Elle s'applique uniquement aux mesures classées dans la catégorie E.

Après avoir passé en revue les critères de mise en œuvre répertoriés dans le système de classification des mesures de l'ESP, on peut les comparer aux caractéristiques de la réforme des politiques agricoles définies par les ministres de l'Agriculture des pays de l'OCDE en $1998^{3}$ et renforcées par l'analyse menée par l'OCDE, qui a donné lieu récemment à une synthèse dans OCDE (2008b). Cette comparaison permet de mieux cerner l'intérêt que présente le système de classification, d'un point de vue analytique, pour suivre l'évolution de l'action publique. Elle aide à déterminer si l'évolution des caractéristiques recensées grâce au système de classification infléchit l'action publique dans le sens de la réforme. Si l'on veut tirer pleinement parti du potentiel analytique offert par le système de classification, il nous faut disposer d'une référence.

Le concept général sur lequel repose la réforme des politiques agricoles implique une allocation des ressources davantage fondée sur les signaux donnés par le marché. Trois caractéristiques - le découplage, le ciblage et l'adaptation — sont pertinentes. " Après avoir, partiellement ou même totalement, découplé le soutien agricole de la production, il est en effet possible d'améliorer encore l'efficacité, l'efficience économique et l'équité en apportant un soutien sous une forme ciblée qui vise directement l'objectif recherché et en adaptant le montant des dépenses au problème à résoudre.»(OCDE, 2008b). Le tableau 3.1 définit ces caractéristiques et illustre de quelle manière il est possible de les classer au sein des catégories et étiquettes.

De façon générale, le système actuel de classification est beaucoup plus performant pour déterminer le degré de découplage que celui des deux autres caractéristiques, en l'occurrence le ciblage et l'adaptation. Néanmoins, ces trois caractéristiques du système ne permettent de prendre en compte qu'une partie de ces mesures. Qui plus est, l'adaptation représente au mieux un lien faible dans la mesure où il concerne également les mesures avec limitation de la production. Il n'en demeure pas moins que ce système est souple et qu'il serait possible d'introduire de nouvelles étiquettes définies en fonction des critères de mise en œuvre afin de refléter la dimension ciblage ou adaptation des mesures gouvernementales.

\footnotetext{
3. Le texte complet du communiqué ministériel est consultable à l'adresse www.oecd.org/agr/ministerial.
} 
Tableau 3.1. Le système de classification des mesures de l'ESP et la réforme des politiques agricoles Exemples illustratifs

\begin{tabular}{|c|c|c|}
\hline \multirow{2}{*}{$\begin{array}{l}\text { Caractéristiques } \\
\text { associées à la réforme } \\
\text { agricole }\end{array}$} & \multicolumn{2}{|c|}{ Éléments permettant la classification dans le système } \\
\hline & Catégories & Étiquettes \\
\hline \multirow[b]{2}{*}{$\begin{array}{l}\text { Découplage - réduit } \\
\text { l'impact des politiques } \\
\text { agricoles sur la } \\
\text { production et les } \\
\text { échanges }{ }^{1}\end{array}$} & \multicolumn{2}{|c|}{ Les progrès réalisés se traduisent par une baisse de la proportion du soutien... } \\
\hline & $\begin{array}{l}\text { - au titre de la production et } \\
\text { d'intrants variables, sans } \\
\text { contraintes sur les intrants } \\
\text { - } \quad \begin{array}{l}\text { au titre des conditions } \\
\text { courantes plutôt que des } \\
\text { conditions non courantes }\end{array} \\
\text { exigeant la production de } \\
\text { produits de base }\end{array}$ & $\begin{array}{l}\text { - liée à la production d'un seul produit } \\
\text { - } \quad \text { assortie de taux des paiements variables } \\
\text { - } \quad \text { avec exceptions concernant les produits } \\
\text { - } \quad \text { sans limitation de la production et/ou des } \\
\text { paiements }\end{array}$ \\
\hline \multirow[b]{2}{*}{$\begin{array}{l}\text { Ciblage - lie les } \\
\text { mesures à des résultats } \\
\text { spécifiques en engageant } \\
\text { des actions précises en } \\
\text { faveur de groupes ou de } \\
\text { zones bien définies }\end{array}$} & \multicolumn{2}{|c|}{ Les progrès réalisés se traduisent par une augmentation de la proportion du soutien... } \\
\hline & $\begin{array}{l}\text { fondée sur des critères non } \\
\text { liés à des produits de base : } \\
\text { présente un lien direct avec } \\
\text { des produits autres que des } \\
\text { produits de base, le retrait de } \\
\text { ressources, etc. }\end{array}$ & $\begin{array}{l}\text { - } \quad \text { avec contraintes volontaires sur les intrants } \\
\text { - fondée sur les revenus/recettes }\end{array}$ \\
\hline \multirow{2}{*}{$\begin{array}{l}\text { Adaptation - prévoit } \\
\text { des paiements } \\
\text { strictement adaptés à } \\
\text { l'obtention de résultats } \\
\text { clairement identifiés }^{2} \\
\end{array}$} & \multicolumn{2}{|c|}{ Les progrès réalisés se traduisent par une augmentation de la proportion du soutien... } \\
\hline & - au titre des recettes & $\begin{array}{l}\text { - avec limitation de la production et/ou des } \\
\text { paiements }{ }^{3} \\
\text { - } \quad \text { avec taux des paiements variables }\end{array}$ \\
\hline \multicolumn{3}{|c|}{$\begin{array}{l}\text { 1. Il est entendu que toutes les mesures de soutien à l'agriculture ont un effet sur les décisions de production, mais à des degrés } \\
\text { divers (OCDE, 2006). }\end{array}$} \\
\hline \multicolumn{3}{|c|}{$\begin{array}{l}\text { 2. Ces deux caractéristiques - ciblage et adaptation - sont parfois combinées. C'est ainsi qu'une étude menée par l'OCDE sur } \\
\text { le ciblage efficace des mesures agricoles indique qu' « Une politique est considérée comme ciblée si elle poursuit des résultats } \\
\text { spécifiques définis dans ses objectifs en identifiant les actions (y compris le niveau et la portée de l'intervention) et/ou les groupes } \\
\text { et/ou les zones les plus appropriés, tout en minimisant les transferts non intentionnels et les retombées négatives. " (OCDE, } \\
\text { 2007a). Aux fins de la présente analyse, elles sont examinées séparément. }\end{array}$} \\
\hline
\end{tabular}

3. Lien relativement faible dans la mesure où cette étiquette permet également de classer les mesures plafonnant la production.

\section{Possibilités analytiques offertes par l'ESP — bilan pratique}

Avant de s'engager dans l'examen plus fin des possibilités qu'offre, du point de vue analytique, le système révisé de classification des mesures de l'ESP par le biais de catégories et d'étiquettes pour suivre et évaluer les politiques agricoles, cette section fait brièvement le point sur son mode d'utilisation pour l'établissement des trois rapports récents suivants: Politiques agricoles des pays de l'OCDE: Suivi et évaluation 2009 (OCDE, 2009a), Panorama des mesures gouvernementales ayant un lien avec le risque (OCDE, 2009b) et «Évolutions des politiques agroenvironnementales des pays de l'OCDE » (chapitre 2 de OCDE, 2009a).

Dans le rapport annuel sur les politiques agricoles des pays de l'OCDE, la composition du soutien aux producteurs est essentiellement présentée et examinée du 
point de vue des catégories de mesures de l'ESP. Un tableau standard de l'estimation du soutien indique la valeur des transferts relevant de chacune des principales catégories et sous-catégories pour deux moyennes triennales (1986-88 et 2006-08), ainsi que les trois dernières valeurs annuelles. Ces données sont fournies pour chaque pays de l'OCDE et pour l'ensemble de la zone de l'OCDE (tableaux 1.3, 1.4 et 3.1, 4.1, 5.1, etc. dans OCDE, 2009a). Le rapport contient également un tableau montrant, pour les mêmes périodes et pays, la composition du soutien aux producteurs par catégorie principale en pourcentage de la valeur de l'ESP, c'est-à-dire que la somme de ces parts atteint $100 \%$ (tableau III.5).

En ce qui concerne la forme graphique, la composition du soutien aux producteurs par catégorie est présentée de six manières différentes dans OCDE (2009a) :

- $\quad$ part en pourcentage des recettes agricoles brutes (c'est-à-dire que la somme des parts indiquée par chaque catégorie représente l'ESP en \%) par an sur la période 1986-2008 pour l'ensemble de l'OCDE (graphique 1.6);

- part en pourcentage de la valeur de l'ESP comparée pour deux moyennes triennales pour tous les pays (graphique 1.7);

- $\quad$ pourcentage des recettes agricoles brutes par an pour chaque pays (graphique 3.2, 4.2, etc.) ;

- $\quad$ pourcentage des recettes agricoles brutes pour une seule moyenne triennale pour tous les pays (graphique $3.1^{4}$ );

- $\quad$ part en pourcentage de l'ESP comparée sur deux moyennes triennales pour tous les pays en faisant ressortir les deux catégories (catégories $\mathrm{E}$ et $\mathrm{F}$ ) correspondant aux paiements n'exigeant pas la production de produits de base (graphique 1.10);

- part en pourcentage de l'ESP comparée sur deux moyennes triennales pour tous les pays et montrant les catégories ayant le plus d'effets de distorsion sur la production et des échanges - représentée par la somme des transferts relevant de l'ESP au titre de la production (catégorie A) et les paiements au titre de l'utilisation d'intrants variables non assortis de contraintes (c'est-à-dire les mesures de la sous-catégorie B1 qualifiées par l'étiquette Sans contraintes sur les intrants) (graphique 1.14).

Dans chacun des trois premiers cas, les sept catégories principales sont présentées, alors que seules certaines de ces catégories le sont dans les trois derniers cas. Dans le graphique 3.1, les catégories $\mathrm{C}$ et $\mathrm{D}$ sont combinées pour donner un total des transferts au titre des $\mathrm{S} / \mathrm{Na} / \mathrm{Rec} / \mathrm{Rev}$ lorsqu'une production est requise. Dans les graphiques 1.10 et 1.1.4, sont surlignées les deux valeurs extrêmes du point de vue de l'impact potentiel du soutien sur la production.

En ce qui concerne les étiquettes, ce sont davantage des tableaux et des graphiques qui sont utilisés pour présenter les résultats, bien que, l'expérience aidant, de nouveaux graphiques soient mis au point chaque année. Le tableau standard de l'estimation du soutien établi pour chaque pays de l'OCDE et pour l'ensemble de la zone de l'OCDE fait apparaître certaines étiquettes comme sous-catégories supplémentaires qui viennent s'ajouter à celles portant sur les recettes/revenus et sur le nombre d'animaux dans la catégorie $\mathrm{C}$, ainsi que sur les taux fixes et variables dans la catégorie $\mathrm{E}$, qui font partie de la classification standard. Ce tableau montre la valeur des transferts résultant des mesures

4. Chaque chapitre par pays débute par le même graphique, où le pays en question figure en caractères gras. 
classées avec contraintes sur les intrants (tant obligatoires que volontaires) dans des catégories B1, B2, B3 et C2, et avec exceptions concernant les produits de base dans les catégories E1 et E2. Il est important de prendre en compte le fait que la valeur des transferts indiqués dans ces tableaux sous une étiquette particulière ne représente pas la valeur totale des transferts qualifiés par ces étiquettes dans l'ESP.

Le rapport donne en revanche la valeur totale des transferts opérés reposant sur un seul produit par pays et pour l'ensemble de la zone de l'OCDE, ventilés par produits (tableaux III.8 à III.21). Il donne également la valeur totale des transferts opérés au titre des $\mathrm{S} / \mathrm{Na} / \mathrm{Rec}$ ou Rev par catégorie pour chacun des pays et l'exprime en part de la valeur de l'ESP (tableaux III.36 à 3.47). Est précisée en outre la proportion totale de l'ESP caractérisée par certaines étiquettes, par pays et pour l'ensemble de la zone de l'OCDE (tableau III.6). Ce tableau indique la part de la valeur de l'ESP avec limitation de la production et des paiements, sans contraintes sur les intrants (tant obligatoires que volontaires), reposant sur des produits individuels et assortis d'une production facultative - bien que cette dernière caractéristique soit déterminée sur la base des catégories ( $\mathrm{E}$ et F) plutôt que sur des étiquettes.

La principale étiquette représentée de manière graphique est le $\mathrm{TSP}^{5}$. La valeur des transferts étiquetés TSP pour chacun des groupes standard de produits est présentée en part en pourcentage des recettes agricoles brutes pour chaque produit, c'est-à-dire que le TSP en \% montre la proportion des recettes brutes perçues pour ce produit et découlant de transferts prévus spécifiquement pour ce produit. Cette valeur est donnée par produit pour l'ensemble de la zone de l'OCDE pour les périodes 1986-88 et 2006-08 (graphique 1.9) et pour chaque pays pour la période 2006-08 (graphiques 3.3, 4.3, etc.). La construction de ces graphiques a pour objectif de montrer la composition des transferts relevant de l'étiquette TSP par catégorie (catégories A1, A2 et autres). L'édition 2009 du rapport Politiques agricoles des pays de l'OCDE: Suivi et évaluation comporte de nouveaux graphiques qui présentent, en pourcentage des recettes agricoles brutes, par an et par pays, le soutien accordé spécifiquement aux produits, c'est-à-dire qu'ils montrent la composition des transferts correspondant aux étiquettes Reposant sur un seul produit, un groupe de produits, tous les produits, ou d'autres transferts aux producteurs (figures 3.4, 4.4, etc.). Une série de graphiques mettant en évidence la contribution des transferts placés sous l'étiquette Avec contraintes sur les intrants a également été incluse (graphique 1.11). Ces graphiques, qui portent sur deux moyennes triennales, indiquent la part en pourcentage de ces transferts dans l'ESP, ainsi que la répartition de ces transferts entre les catégories principales, mesurés en pourcentage des recettes agricoles brutes, afin de donner une idée de leur importance relative.

Dans Panorama des mesures gouvernementales ayant un lien avec le risque (OCDE, $2009 b$ ), le système révisé de classification des mesures de l'ESP aide à répertorier les mesures liées aux risques au sein de l'ESP. Toutefois, comme le système de classification repose sur des critères de mise en œuvre et non sur des objectifs d'action, cette tâche n'est

5. L'importance relative de l'étiquette TSP se traduit également par le fait que les indicateurs de soutien spécifiques sont obtenus à partir de cette étiquette, en l'occurrence les transferts aux producteurs reposant sur un seul produit (TSP aux producteurs) — valeur monétaire annuelle des transferts bruts des consommateurs et des contribuables aux producteurs agricoles, mesurés au départ de l'exploitation, qui découlent des mesures liées à la production d'un seul produit, de sorte que le producteur doit produire ce produit pour percevoir le paiement. Cet indicateur nominal sert à calculer deux indicateurs proportionnels/en pourcentage - la part des TSP dans l'ESP totale et les TSP en pourcentage. 
ni évidente, ni simple. Le critère de mise en œuvre qui s'est révélé le plus utile est l'étiquette relative aux taux variables, qui sert à déterminer les mesures à caractère contracyclique dans quatre catégories : A2 (CO), C (PC), D (PHR) et E (PHNR). Toutes les mesures liées aux risques prises en compte dans l'ESP n'ont toutefois pas pu être répertoriées, et nombre d'entre elles n'ont pu être relevées que grâce à un examen ligne par ligne des différentes mesures figurant dans la base de données. Alors que certaines formes de mesures liées aux risques se trouvaient classées dans la même catégorie pour tous les pays, par exemple les services de vulgarisation dans la catégorie B3 (PIS), d'autres ont été découvertes dans plusieurs catégories, par exemple les transferts aux producteurs résultant de mesures d'aide en cas de calamités, ont été classés dans trois catégories différentes, en l'occurrence B1 (PIV), B2 (PIF) et C (PC).

Le système révisé de classification des mesures de l'ESP a également servi à identifier les paiements agroenvironnementaux dans un chapitre spécial du rapport 2009 Politiques agricoles des pays de l'OCDE: Suivi et évaluation (OCDE, 2009a). Ce chapitre porte sur tout l'éventail des mesures à visée agroenvironnementale, notamment les exigences réglementaires, les taxes et redevances environnementales, les droits et quotas échangeables, et les approches et paiements locaux. La base de données des ESP ne permet de recenser que les paiements agroenvironnementaux du fait que les réglementations, taxes et redevances environnementales et les autres approches ne sont pas intégrées à cette base. On peut néanmoins trouver des informations sur tout l'éventail des mesures agroenvironnementales, par pays et par objectif environnemental, dans la base de données de l'OCDE Inventaire des mesures à caractère environnemental en agriculture (www.oecd.org/tad/env/inventory).

Ce chapitre conforte l'idée selon laquelle il n'est pas évident de localiser les mesures conçues pour atteindre des objectifs environnementaux grâce au système de classification des mesures de l'ESP, même si cette localisation est probablement plus facile dans ce cas que pour les mesures liées au risque. Pour recenser les paiements agroenvironnementaux, on fait appel tout à la fois à des catégories et des étiquettes : catégories F1 (PNLT) et F2 (PNSO) ; et catégories B1 (PIV), B2 (PIF), B3 (PIS), C (PC) et D (PHR), dans lesquelles on leur attribue également l'étiquette avec contraintes sur les intrants, c'est-à-dire que ces mesures imposent aux agriculteurs de réduire leur consommation d'intrants, d'appliquer certaines pratiques agricoles ou de respecter certains critères ${ }^{6}$. Il n'en reste pas moins que toutes les mesures classées dans ces catégories et qualifiées par ces étiquettes n'ont pas d'objectifs environnementaux assignés. Le système de classification est utile pour repérer la localisation générale des mesures, mais là encore, un examen ligne par ligne de chaque mesure est nécessaire.

Une fois les mesures pertinentes identifiées dans la base des ESP, leur analyse est relativement similaire dans les deux études. Celles-ci présentent toutes deux sous forme graphique la somme des transferts associés aux mesures en question, exprimée en part de l'ESP et en part des paiements budgétaires totaux (ESP moins SPM), et commentent les évolutions de ces parts dans le temps, ainsi que les disparités entre pays. L'étude sur les risques compare deux moyennes sur six ans (1992-97 et 2002-07), tandis que l'étude sur

6. Dans ce rapport, les paiements subordonnés à l'écoconditionnalité et les paiements aux zones défavorisées ne sont pas considérés comme des paiements agroenvironnementaux. Dans le cadre du projet sur l'Inventaire des mesures à caractère environnemental en agriculture, qui a été engagé sous la houlette du groupe de travail mixte sur l'agriculture et l'environnement, l'OCDE poursuit sa réflexion en vue de déterminer si l'on peut considérer comme paiements agroenvironnementaux les paiements dédiés aux zones défavorisées. 
les questions agroenvironnementales donne la part annuelle sur une période de 13 années (1996 à 2008). Ces deux études indiquent par ailleurs, en la commentant, la composition des mesures gouvernementales. Tandis que l'étude sur les mesures agroenvironnementales analyse la composition des transferts selon cinq combinaisons de catégorie/étiquette, l'étude sur les risques retient une autre composition, qui se rapporte plus spécifiquement au risque - réduction des risques, atténuation/maîtrise ex ante des risques, etc.

\section{Le potentiel analytique de l'ESP — Autres considérations pratiques}

La présente section analyse les informations contenues dans la base de données des ESP d'un double point de vue, à savoir les catégories par étiquette et par pays, et les pays par catégorie et par étiquette. Même si les informations fournies se chevauchent en partie, analyser les données de ces différents points de vue garantit une véritable prise en compte de toutes les dimensions de la base de données. Il y aura également chevauchement partiel avec l'analyse présentée dans la section précédente, en particulier en ce qui concerne les informations données dans le rapport annuel Politiques agricoles des pays de l'OCDE. Ce qui importe, cependant, c'est de se demander si les combinaisons de catégories et d'étiquettes présentées sont bien les plus appropriées. L'analyse porte sur les données annuelles enregistrées entre 1986 et 2007, ce qui permet de s'assurer de la prise en compte des catégories et étiquettes utilisées au cours des différentes années, en dehors des périodes 1986-88 et 2005-07, telles qu'indiquées dans le rapport 2008. L'analyse est effectuée au niveau des sous-catégories, à deux exceptions près : en premier lieu, les deux sous-catégories de $\mathrm{C}$ et $\mathrm{E}$ sont considérées comme des étiquettes plutôt que comme des sous-catégories, et en second lieu, les sous-catégories F1, F2 et F3 ne sont pas considérées comme telles, la catégorie $\mathrm{F}$ étant considérée comme une catégorie principale.

Les données assemblées peuvent donner lieu à de multiples permutations et combinaisons. Il existe en fait 514 combinaisons possibles entre catégories et étiquettes, et 144 possibilités de combinaisons d'étiquettes pour les deux catégories $\mathrm{C}$ et $\mathrm{D}$. Le grand nombre de combinaisons catégories/étiquettes envisageable rend l'analyse bidimensionnelle extrêmement difficile. Néanmoins, il n'existe par définition aucune mesure étiquetée comme ouvrant droit à des paiements à la fois au titre du nombre d'animaux et de tous les produits en général - ce qui réduit le nombre total de combinaisons possibles de 24 (12 chacune pour les catégories C et D). Par ailleurs, certaines combinaisons revêtent davantage d'importance dans la composition de l'ESP. En 2006 et 2007, plus de la moitié de l'ESP totale pour l'ensemble de la zone de l'OCDE était imputable à deux combinaisons de catégories et étiquettes seulement : catégorie A1 (MPS) assortie des étiquettes relatives à l'absence de limites, à des taux des paiements variables et à un seul produit (40\%), et la catégorie E (PHNR) assortie des étiquettes «avec limites de la production et/ou des paiements », « avec taux des paiements fixes », «avec contrainte sur les intrants », « reposant sur les superficies» et «avec exceptions concernant la production » $(12 \%)$.

\subsection{Catégories par étiquette et par pays}

Les tableaux annexes 1 à 10 de l'annexe 2 indiquent, pour chaque catégorie de mesures de l'ESP, la proportion des transferts qui ont été affectés à chacune des étiquettes appropriées, par pays et pour l'ensemble de la zone de l'OCDE. Ils permettent ainsi de se 
faire une idée de l'utilisation et de l'importance relatives des étiquettes au sein des différentes catégories et entre catégories. Le tableau 2.3 récapitule les principales conclusions qui s'en dégagent.

Il est aisé de construire un graphique représentant l'évolution de la composition du soutien classé dans une catégorie particulière telle qu'elle ressort des étiquettes (graphique 5.1). En effet, les étiquettes, lorsqu'elles s'appliquent, sont intégralement assignées à une catégorie particulière, ce qui permet des comparaisons. La catégorie $\mathrm{C}$ a été choisie comme exemple, parce que c'est elle qui réunit le plus grand nombre de combinaisons d'étiquettes au sein d'une catégorie, ce qui met en évidence à la fois l'importance du nombre de combinaisons qu'il est théoriquement possible de constituer et la diversité des mesures classées dans cette catégorie. De toutes les catégories de mesures de l'ESP, c'est la catégorie $C$ qui a peut-être vu sa composition le plus sensiblement modifiée.

Cinq groupes d'étiquettes peuvent être appliquées à la catégorie $\mathrm{C}$, l'une des variables étant choisie pour représenter chacun de ces cinq groupes sur l'axe du graphique en radar. Deux des étiquettes autorisent seulement deux options définissant 1) si les transferts sont assortis d'une limitation de la production et/ou des paiements ou $2^{\circ}$ si le taux des paiements est variable ou fixe. Dans l'un et l'autre cas, choisir l'une de ces variables pour l'axe revient à dire que le reste des transferts relève de l'autre variable. Par exemple, le graphique 2.1 montre le pourcentage des transferts classés dans la catégorie $\mathrm{C}$ et assortis des étiquettes avec limitation (L) et taux fixe (F). En soustrayant ce pourcentage de $100 \%$, on obtient le pourcentage des transferts opérés dans le cadre de mesures ne s'accompagnant d'aucune limitation ou assorties d'un taux de paiement variable.

Les trois autres groupes d'étiquettes offrent deux options ou plus. Il faut être alors particulièrement attentif à la sélection de l'étiquette (ou de la combinaison d'étiquettes) choisie pour interpréter les résultats. Pour l'étiquette indiquant l'élément au titre duquel le transfert est opéré, le graphique montre le pourcentage des transferts qualifiés par l'étiquette Reposant sur les recettes ou le revenu (R/I), c'est-à-dire que les deux étiquettes ont été regroupées. Cette combinaison fait sens, puisque ces deux étiquettes sont étroitement liées, toutes deux ayant trait aux performances financières. Le reste représente la proportion des transferts qualifiés par l'étiquette Reposant sur les superficies ou le nombre d'animaux, ces deux possibilités concernant des facteurs de production. On trouve souvent ces quatre variables réunies dans ces deux groupes, ce qui correspond en fait au mode d'organisation des sous-catégories de $\mathrm{C}$, d'une part en $\mathrm{C} 1$ pour les recettes et le revenu et en $\mathrm{C} 2$ pour les superficies et le nombre d'animaux.

De la même manière, pour l'étiquette permettant de déterminer s'il y a contraintes sur les intrants, le graphique montre le pourcentage des transferts étiquetés avec contraintes sur les intrants, soit obligatoires, soit volontaires (IC). Dans ce cas, le reste correspond à la part des transferts sans contraintes sur les intrants. Ce que le graphique n'est pas en mesure de mettre en évidence, c'est la proportion des transferts opérés au sein d'un groupe, c'est-à-dire la part des transferts avec contraintes obligatoires sur les intrants par rapport aux contraintes volontaires.

Enfin, en ce qui concerne l'étiquette indiquant quels produits donnent lieu à un soutien, le graphique montre le pourcentage des transferts auxquels a été accolée l'étiquette Reposant sur tous les produits (AC). Le reste des transferts repose donc sur des produits individuels ou des groupes de produits. Ces deux types de transferts ont été regroupés parce qu'ils sont tous deux beaucoup plus spécifiques que les transferts auxquels ouvrent droit tous les produits. 
Tableau 5.1. Importance des étiquettes accolées aux catégories de mesures

\begin{tabular}{|c|c|}
\hline $\begin{array}{c}\text { Catégorie } \\
\text { de } \\
\text { mesure } \\
\text { de l'ESP }\end{array}$ & Principales conclusions \\
\hline A1. MPS & $\begin{array}{l}\text { La seule différence réelle qui apparaisse entre les pays concerne la limitation de la production et/ou } \\
\text { des paiements. L'Australie, la Corée, I'Islande, le Japon et la Nouvelle-Zélande n'ont jamais pris } \\
\text { aucune mesure de plafonnement, tandis que tous les transferts au titre du MPS ont toujours été limités } \\
\text { aux États-Unis et en Turquie. La part des transferts avec limitation a augmenté au Canada, mais elle a } \\
\text { diminué dans l'UE et en Suisse. } \\
\text { Le seul pays à indiquer des taux de paiement fixes pour les transferts au titre du MPS est le Canada ; } \\
\text { tous les autres pays ont défini des taux variables. }\end{array}$ \\
\hline A2. PO & $\begin{array}{l}\text { Dans la plupart des pays, la proportion des transferts avec limitation de la production et/ou des } \\
\text { paiements progresse, excepté aux États-Unis, au Japon et en Norvège où, à l'inverse, c'est la } \\
\text { proportion des transferts avec limitation qui augmente. } \\
\text { Des disparités entre pays existent pour les taux de paiement. En Corée, en Islande, en Norvège, en } \\
\text { Suisse et en Turquie, les taux de paiement ont toujours été fixes, alors qu'ils ont toujours été variables } \\
\text { aux États-Unis et au Canada. Le Japon et l'UE sont passés de taux variables à des taux fixes, et le } \\
\text { Mexique a abandonné les taux fixes pour des taux variables. } \\
\text { La majeure partie des pays n'impose aucune contrainte sur les intrants, à l'exception des États-Unis } \\
\text { (qui ont toujours imposé des contraintes obligatoires), l'UE (où des contraintes obligatoires ont été } \\
\text { mises en place sur certains transferts) et en Suisse (où les contraintes volontaires ont laissé la place à } \\
\text { une absence de contraintes). }\end{array}$ \\
\hline B1. PIV & $\begin{array}{l}\text { Dans tous les pays, la vaste majorité de ces transferts sont versés sans limitation de la production } \\
\text { et/ou des paiements. Seul le Mexique semble tenter d'imposer des limites sur ces formes de soutien. } \\
\text { Les taux de paiement définis pour ces transferts sont généralement fixes, excepté en Australie, au } \\
\text { Japon et en Norvège, où la quasi-totalité, voire l'ensemble des transferts, sont assortis de taux } \\
\text { variables. } \\
\text { Ces transferts sont presque toujours octroyés sans contraintes sur les intrants, exception faite des } \\
\text { États-Unis et de la Suisse, où une faible proportion est soumise à des contraintes. } \\
\text { Dans la plupart des pays, les mesures relevant de cette catégorie ont toujours été offertes pour } \\
\text { l'ensemble des produits. Au Canada, au Mexique, en Norvège et dans l'UE, une proportion de plus en } \\
\text { plus grande de paiements au titre de groupes de produits est désormais offerte. }\end{array}$ \\
\hline B3. PIS & $\begin{array}{l}\text { Dans tous les pays, à l'exception du Canada et des États-Unis, ces transferts sont octroyés sans } \\
\text { limitation de la production et/ou des paiements. Aux États-Unis, un tiers des transferts relevant de } \\
\text { cette catégorie sont plafonnés. } \\
\text { De la même manière, dans tous les pays à l'exception des États-Unis, les taux de paiement } \\
\text { applicables sont fixes. Aux États-Unis, une faible proportion des paiements repose sur des taux } \\
\text { variables. } \\
\text { Dans la quasi-totalité des pays, aucune contrainte sur les intrants n'est imposée, à l'exception des } \\
\text { États-Unis et de l'UE, qui ont mis en place des contraintes obligatoires sur certains transferts. } \\
\text { Alors que la plupart des mesures entrant dans cette catégorie s'appliquent à l'ensemble des produits, } \\
\text { la proportion des transferts opérés dans le cadre de cette catégorie en faveur de produits individuels ou } \\
\text { de groupes de produits a été plus importante (environ } 25 \% \text { au niveau de l'OCDE) que pour les } \\
\text { catégories B1 ou B2. Tous les pays, sauf le Canada, offrent certains transferts au titre des PIS pour } \\
\text { des produits individuels ou des groupes de produits, tandis qu'en Norvège et en Turquie, la totalité de } \\
\text { ces transferts va à des produits individuels ou des groupes de produits. }\end{array}$ \\
\hline
\end{tabular}




\begin{tabular}{c|c}
\hline $\begin{array}{c}\text { Catégorie } \\
\text { de } \\
\text { mesure } \\
\text { de l'ESP }\end{array}$ & Principales conclusions \\
\hline B2. PIF & Dans tous les pays, à l'exception des États-Unis, ces transferts sont octroyés sans limitation de la
\end{tabular}

production et/ou des paiements. Aux États-Unis, tous les transferts relevant de cette catégorie sont plafonnés.

Les taux de paiement sont généralement fixes, sauf en Australie et en Suisse où, dans leur quasitotalité, ils sont assortis de taux variables. Les États-Unis, la Norvège et l'UE sont passés de taux variables à des taux fixes.

Aucune contrainte sur les intrants n'est imposée dans la majeure partie des pays, à l'exception du Canada (qui a mis en place des programmes avec contraintes volontaires), la Corée et l'UE (où des contraintes obligatoires ont été introduites pour certains transferts) et aux États-Unis (qui ont toujours exigé une forme ou une autre de contraintes).

Dans la plupart des pays, les transferts au titre du PIF sont généralement offerts pour tous les produits, sauf en Islande et en Turquie, où ils sont dédiés soit à des produits individuels, soit à des groupes de produits.

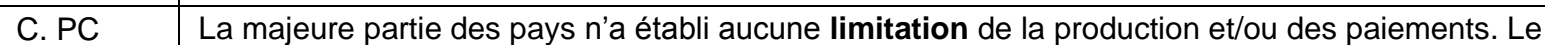
Canada, le Japon et l'UE semblent s'orienter vers un plafonnement, alors que les États-Unis et la Suisse versent leurs paiements sans limitation.

L'Australie, l'Islande, la Norvège et la Suisse ont recours uniquement à des taux de paiement fixes. Tous les autres pays utilisent tant des taux fixes que des taux variables. Aux États-Unis, on observe au fil du temps une augmentation progressive de la proportion des transferts fondés sur des taux variables.

Les transferts sont octroyés sans contraintes en Australie, au Canada, au Mexique, en NouvelleZélande et en Turquie, mais aucun pays ne subordonne l'ensemble des transferts à des contraintes. Les États-Unis, le Japon, la Norvège, la Suisse et l'UE accroissent progressivement la proportion des transferts qu'ils versent avec contraintes.

Seule la Nouvelle-Zélande prévoit des transferts relevant de cette catégorie au titre de tous les produits. En Islande, ces transferts sont octroyés uniquement au titre des produits individuels, tandis que la Suisse les consacre essentiellement aux groupes de produits. Tous les autres pays ont adopté des formules hybrides. Les États-Unis, le Japon, le Mexique, la Norvège et l'UE augmentent progressivement au fil des ans les transferts reposant sur tous les produits.

De façon générale, les pays font appel à plusieurs variables $\mathbf{S} / \mathrm{Na} / \mathbf{R e c} / \mathbf{R e v}$ pour définir leurs transferts. L'Australie et la Nouvelle-Zélande prévoient le versement de transferts uniquement sur la base du revenu, tandis qu'aux États-Unis, au Canada, au Japon et en Norvège, une proportion de plus en plus importante de transferts est octroyée soit au titre des revenus, soit au titre des recettes. Ce sont essentiellement les pays européens qui ont recours à des transferts au titre du nombre d'animaux, même si, par rapport aux transferts au titre des superficies, ces transferts ont perdu du terrain dans I'UE, mais pas en Norvège ni en Suisse.

D. PHR $\quad$ L'Australie, le Mexique, la Nouvelle-Zélande et la Suisse n'imposent aucune limitation de la production et/ou des paiements. À l'inverse, le Canada et l'Islande plafonnent tous les transferts, tandis que la Norvège applique les deux types de mesures.

Ces transferts sont presque toujours assortis de taux de paiement fixes. Seul le Canada leur applique des taux variables.

En Australie, au Canada et en Islande, ces transferts ne sont subordonnés à aucune contrainte sur les intrants. La Suisse impose sur tous ces transferts des contraintes obligatoires, alors qu'à l'inverse, le Mexique leur applique systématiquement des contraintes volontaires.

En Australie, en Islande, au Mexique et en Norvège, ces transferts sont octroyés au titre de produits individuels, alors qu'ils bénéficient à des groupes de produits au Canada, en Nouvelle-Zélande et en Suisse. Seuls le Canada, la Norvège et la Suisse consacrent une partie de ces transferts à l'ensemble des produits.

De façon générale, les pays font appel à une seule variable $\mathbf{S} / \mathbf{N a} / \mathbf{R e c} / \mathbf{R e v}$ pour établir leurs transferts : I'Islande, le Mexique et la Suisse utilisent le nombre d'animaux, tandis que l'Australie, le Canada et la Norvège ont opté pour les recettes ou les revenus, la Norvège faisant par ailleurs exception puisqu'elle utilise également les superficies. 


\begin{tabular}{|c|c|}
\hline $\begin{array}{c}\text { Catégorie } \\
\text { de } \\
\text { mesure } \\
\text { de l'ESP }\end{array}$ & Principales conclusions \\
\hline E. PHNR & $\begin{array}{l}\text { Des limites sont imposées sur tous les transferts en Corée, aux États-Unis, au Mexique, en Turquie et } \\
\text { dans l'UE. En revanche, aucun transfert n'est soumis à une quelconque limite en Australie, en Islande } \\
\text { et en Suisse. Une part des transferts, faible mais en progression, est plafonnée au Canada et au } \\
\text { Japon. } \\
\text { La vaste majorité des transferts est assortie d'un taux de paiement fixe. Seuls, l'Australie, le Canada } \\
\text { et les États-Unis ont recours à des taux variables. } \\
\text { La grande majorité des pays verse ces transferts sans contraintes sur les intrants. En Suisse et dans } \\
\text { l'UE, les transferts sont toujours soumis à des contraintes obligatoires (conditionnalité), et ils le sont la } \\
\text { plupart du temps aux États-Unis. } \\
\text { De façon générale, les pays ont recours à une seule des variables S/Na/Rec/Rev pour définir leurs } \\
\text { transferts, les superficies étant la plus courante : la Corée, les États-Unis, le Japon, le Mexique, la } \\
\text { Suisse, la Turquie et l'UE utilisent les superficies, tandis que l'Islande et, dans une moindre mesure, le } \\
\text { Canada, utilisent le nombre d'animaux, l'Australie et le Canada (qui se basaient traditionnellement sur } \\
\text { les superficies) se fondent sur les recettes ou les revenus. } \\
\text { La plupart des transferts sont assortis d'exceptions. Seuls le Canada, la Corée, la Suisse et la Turquie } \\
\text { ont toujours offert des transferts sans exceptions. Tous les autres pays ont mis en œuvre quelques } \\
\text { mesures, voire toutes, avec exceptions. Avec la réforme de 2003, I'UE a subordonné l'octroi de } \\
\text { certains paiements à des exceptions qui seront par la suite progressivement abandonnées.7 Aux États- } \\
\text { Unis, les paiements introduits en } 1996 \text { sont assortis d'exceptions, mais en fin de période, la part des } \\
\text { paiements non assortis d'exceptions semble quelque peu progresser. }\end{array}$ \\
\hline F. PN & $\begin{array}{l}\text { La seule étiquette capable de faire apparaître les disparités entre pays concerne l'imposition ou non } \\
\text { d'une limitation de la production et/ou des paiements. Toutes les autres étiquettes, soit ne sont pas } \\
\text { applicables, soit sont prédéterminées par définition (contraintes volontaires sur les intrants, par } \\
\text { exemple). Au cours de la période considérée, huit pays ont mis en œuvre des mesures classées dans } \\
\text { cette catégorie. Dans tous les cas, ces mesures se sont vu assigner l'étiquette sans limitation. } \\
\text { Cependant, la caractéristique réellement importante des mesures relevant de cette catégorie concerne } \\
\text { les critères non liés à des produits de base ciblés par la mesure considérée, c'est-à-dire que ce sont } \\
\text { les sous-catégories F1, F2 et F3 qui vont différencier les pays. Dans ce cas précis, les sous-catégories } \\
\text { sont beaucoup plus utiles que les étiquettes pour le classement des mesures. }\end{array}$ \\
\hline
\end{tabular}

7. Sur la période 2005-07, la réduction progressive de la part des paiements assortis d'exceptions résulte de la progression des paiements uniques sans exception dans les nouveaux pays membres et dans les pays qui ont choisi une mise en œuvre dynamique du régime de paiement unique. En outre, la réforme du secteur des fruits et légumes introduite en 2008 entraînera la disparition des exceptions en 2011, fin de la période de mise en œuvre. 


\section{Graphique 5.1. Évolution de la composition de la catégorie $C$ par étiquette pour différents pays,} 1986-88 et 2005-07

Canada

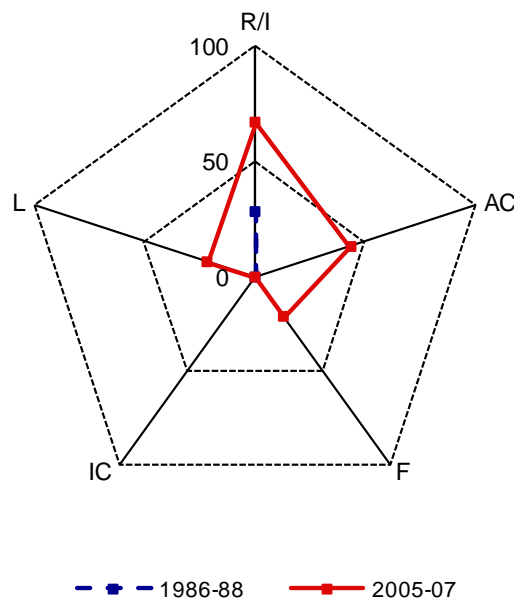

Suisse

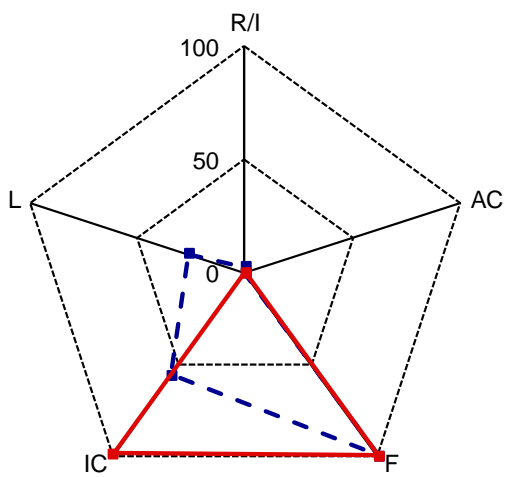

$--1986-88 \longrightarrow 2005-07$
UE27

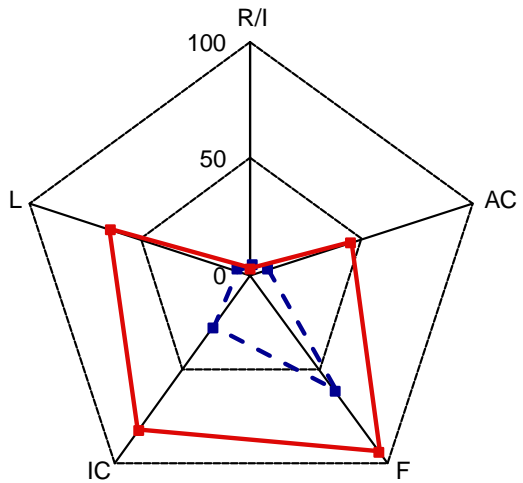

$--1986-88 \longrightarrow 2005-07$

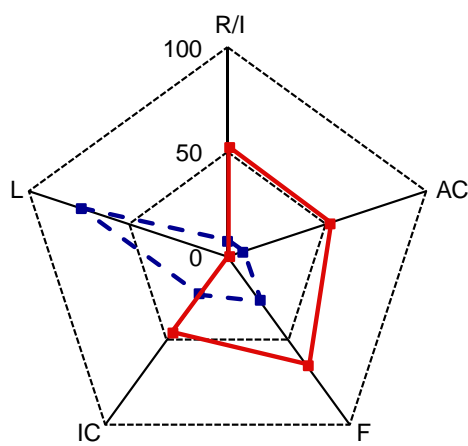

$--1986-88 \longrightarrow$ 2005-07

Symboles : A/An - Superficies/Nombre d'animaux; "AC" — ensemble des produits ; "F" — taux de paiement fixe ; "IC" — avec contraintes sur les intrants (tant obligatoires que volontaires) ; "L" — avec limitation de la production et/ou des paiements.

Si l'on a opté pour telle étiquette ou tel groupe d'étiquettes, c'est aussi parce que la progression de la part des transferts qui leur sont attribués pourrait bien illustrer un infléchissement vers une réforme des politiques agricoles telle qu'évoquée dans le tableau 3.1. Plus le tracé est centré au milieu du diagramme, plus grande est la proportion des transferts accordés à des produits individuels ou des groupes de produits au titre de facteurs de production (terre ou nombre d'animaux), sur la base des conditions courantes du marché, sans limitation et sans contraintes sur les intrants. À l'inverse, plus le tracé est 
excentré, plus grande est la proportion des transferts accordés à l'ensemble des produits, sur la base des résultats financiers, assortis de taux fixes, sans limitation ni contraintes.

Au cours de la période 1986-88, les mesures canadiennes relevant de la catégorie C présentaient toutes les caractéristiques suivantes : sans limitation, sans contraintes, avec taux des paiements variables et accordées au titre de produits individuels ou de groupes de produits, la majorité d'entre elles reposant sur les superficies/nombre d'animaux. La situation était plus ou moins analogue aux États-Unis, mais la majorité des transferts y était plafonnée et certains étaient assortis de taux de paiement fixes et de contraintes sur les intrants. En Suisse et dans l'UE, les contraintes sur les intrants et les taux de paiement fixes étaient relativement plus importants.

Une comparaison entre 1986-88 et 2005-07 fait ressortir diverses similitudes et différences entre pays pour la composition de la catégorie C. En partant du haut du diagramme et en tournant dans le sens des aiguilles d'une montre, on observe que le Canada et les États-Unis font reposer une proportion plus grande de leurs transferts sur les revenus/recettes, tandis qu'en Suisse et dans l'UE, ce sont les paiements en fonction des superficies ou du nombre d'animaux qui prédominent. Les paiements accordés à l'ensemble des produits sont de plus en plus nombreux au Canada, aux États-Unis et dans l'UE, alors qu'en Suisse, les paiements demeurent fondés sur des produits individuels ou des groupes de produits. La proportion de paiements fixes a augmenté au Canada, aux États-Unis et dans l'UE, tandis qu'elle est demeurée stable en Suisse. Les paiements désormais subordonnés à des contraintes sur les intrants gagnent du terrain aux ÉtatsUnis, en Suisse et dans l'UE, mais le Canada ne les imposent pas encore. Enfin, au Canada et dans l'UE, une proportion importante des transferts est assortie de limitation de la production et/ou des paiements, alors qu'aucun plafonnement n'existe aux États-Unis ou en Suisse.

\subsection{Pays par catégorie et étiquette}

On peut analyser la composition du soutien au niveau national en étudiant la proportion des mesures de soutien classées dans les diverses catégories ou auxquelles a été attribuée telle ou telle étiquette, et ce pour des pays ou groupes de pays particuliers. Cette opération permet de se faire une idée de l'utilisation et de l'importance relatives des catégories et étiquettes, tant au niveau d'un pays qu'entre pays. Cette démarche est celle qui est communément adoptée pour analyser la composition du soutien dans le rapport annuel Politiques agricoles des pays de l'OCDE, comme on l'a vu dans la section 4.

Les tableaux annexes 10 à 22 indiquent, pour chaque pays de l'OCDE et pour l'ensemble de la zone de l'OCDE, la proportion des transferts qui ont été classés dans les catégories et auxquels on a accolé diverses étiquettes pour chacune des années comprises entre 1986 et 2007. Là encore, toutes les années ont été prises en compte, et pas simplement la première et la dernière moyennes triennales, afin de mettre en évidence l'évolution de ces proportions au cours des années concernées. La composition du soutien n'évolue pas dans le temps de manière linéaire depuis la première période considérée jusqu'à la dernière. Il arrive en effet qu'au cours des années où elles ont été appliquées, des mesures soient mises en œuvre alors qu'elles relèvent de catégories et/ou d'étiquettes qui ne sont pas représentées dans les première et dernière périodes triennales. Même avec des points de départ et d'arrivée similaires, les pays peuvent avoir des trajectoires de réforme extrêmement différentes. 
La moitié supérieure de chaque tableau donne la composition du soutien aux producteurs par catégorie. Étant donné que le rapport annuel Politiques agricoles des pays de l'OCDE fournit la composition catégorie par catégorie, la présente analyse s'intéresse à des ensembles de catégories (voir section 3) regroupées en fonction de l'élément au titre duquel le soutien est accordé (production, intrants, superficies/nombre d'animaux/recettes/revenus et critères non liés à des produits de base), selon que la base de calcul est courante ou non courante (niveau antérieur ou fixe), et selon que la production de produits de base est requise ou facultative. La somme de ces différentes composantes est égale à $100 \%$.

Dans l'optique de révéler les interactions entre les catégories et les trois étiquettes examinées dans la section 3, la moitié supérieure du tableau comprend également les étiquettes correspondantes. En ce qui concerne l'élément pris en compte pour consentir le soutien, le tableau ventile le total allant aux $\mathrm{S} / \mathrm{Na} / \mathrm{Rec} / \mathrm{Rev}$, qui correspond à la somme des proportions classées dans les catégories $\mathrm{C}, \mathrm{D}$ et $\mathrm{E}$, entre chacune de ces variables. L'étiquette correspond à un sous-ensemble de cet ensemble de catégories. Dans ce cas précis, la somme des étiquettes est égale à la proportion totale des transferts accordés au titre des S/Na/Rec/Rev. Prenons l'exemple du Canada : en 1986, $28 \%$ du soutien aux producteurs versés au titre des $\mathrm{S} / \mathrm{Na} / \mathrm{Rec} / \mathrm{Rev}$ se décomposent en $16 \%$ au titre des superficies, $0.6 \%$ au titre du nombre d'animaux et $11.3 \%$ au titre des recettes.

S'agissant de l'obligation ou non de produire des produits de base, l'étiquette indiquant le droit à paiement en fonction de la relation avec les produits précise de quelle production particulière il s'agit : un produit individuel (SC), un groupe de produits (GC) ou tous les produits (AC). On peut considérer cette étiquette comme un sous-ensemble de ce vaste groupe de catégories. Dans ce cas précis, la somme des étiquettes (SC, GC et $\mathrm{AC}$ ) est égale à la proportion totale des transferts répertoriés comme exigeant la production de produits de base. Si l'on reprend l'exemple du Canada, $97.7 \%$ du soutien versé aux producteurs avec obligation de produire en 1986 se décomposent en $71.5 \%$ subordonnés à la production de produits individuels, $14 \%$ à celle de groupes de produits et $12.2 \%$ à celle de tous les produits.

La situation est légèrement différente dans le cas de la troisième étiquette complétant les catégories. Les catégories peuvent être regroupées en fonction de la base de calcul, courante ou non courante. La variable relative aux taux de paiement apporte une précision supplémentaire, à savoir si le taux est variable (courant) ou fixe. Toutefois, au lieu de constituer une sous-catégorie d'un groupement particulier de catégories comme c'est le cas pour les étiquettes précédentes, les deux options possibles peuvent être appliquées aux deux groupements. Il est donc possible de combiner groupement de catégories et étiquettes pour obtenir des informations sur la part du soutien aux producteurs reposant sur un niveau effectif et assorti de taux de paiement déterminés par les conditions courantes, ou reposant sur un niveau effectif et assorti de taux de paiement fixes, etc. La cinquième combinaison catégorie/étiquette représente les mesures de soutien répertoriées comme reposant sur un niveau effectif, mais pour lesquelles l'étiquette relative aux taux de paiement n'est pas applicable. Dans ce cas précis, elle est équivalente au groupe des catégories concernant les produits autres que des produits de base, c'est-à-dire à la valeur des transferts découlant de mesures relevant de la catégorie F.

La moitié inférieure de chaque tableau rend compte de la composition du soutien aux producteurs par étiquette. Les trois étiquettes utilisées en conjonction avec les groupements de catégories de la moitié supérieure du tableau sont reprises pour rappel. Pour certains pays, $100 \%$ du soutien aux producteurs relèvent de l'une de ces étiquettes, 
ce qui reflète uniquement la situation particulière de ce pays. Les étiquettes additionnées n'atteignent pas nécessairement $100 \%$, ce qui est très fréquent.

Une comparaison des tableaux permet de faire ressortir les caractéristiques générales suivantes concernant l'utilisation relative des catégories et étiquettes :

- Le recours aux critères de mise en œuvre représentés par les catégories et les étiquettes n'a cessé d'augmenter au fil des ans, autrement dit la répartition des transferts entre les différentes catégories et étiquettes investit de plus en plus de colonnes. Cette évolution n'a rien de surprenant étant donné la complexité croissante des mesures et dans la mesure où le système de classification le permet.

- Les trois étiquettes directement liées aux catégories améliorent sensiblement le niveau de détail fourni au niveau des catégories.

- Les proportions relatives se modifient souvent en fonction de l'évolution du MPS. Ce dernier représente généralement la catégorie la plus importante de transferts, et dans la mesure où l'étiquette utilisée par les différents pays varie relativement peu (se reporter à la section précédente), l'évolution du MPS influe notablement sur les modifications au sein des trois groupes de catégories ainsi que des étiquettes indiquant une limitation de la production et/ou des paiements, un paiement au titre de produits individuels et l'application de taux de paiement variables.

- Dans le cas de l'étiquette indiquant que les paiements sont fondés sur les $\mathrm{S} / \mathrm{Na} / \mathrm{Rec} / \mathrm{Rev}$, alors que les variables font de fait ressortir les différences d'approche de certains pays au fil du temps, en particulier au Canada, cette étiquette semble mettre plus fortement en évidence les disparités entre pays.

- De la même manière, alors que l'étiquette relative aux contraintes sur les intrants indique l'évolution dans le temps des approches adoptées par des pays tels que la Suisse ou l'UE, il semble qu'elle pointe encore plus nettement les différences entre pays.

Les trois combinaisons catégorie/étiquette mettent en lumière certaines des principales modifications des politiques agricoles. Le graphique 5.1 montre l'évolution de ces trois combinaisons dans le cas de la Suisse. Le cercle intérieur indique la situation pour la période 1986-88, tandis que le cercle extérieur fait apparaître la situation pour 2005-07, l'ensemble des deux cercles apportant un éclairage particulièrement utile sur l'évolution de l'action gouvernementale. En ce qui concerne l'élément au titre duquel le soutien est accordé, on observe que les transferts au titre de la production ont été délaissés au profit des transferts reposant sur les superficies et le nombre d'animaux, et l'on constate une augmentation de la proportion des transferts accordés aux producteurs pour des produits autres que des produits de base. S'agissant du lien avec des facteurs courants, la proportion du soutien octroyé en fonction des niveaux effectifs et assortis de taux de paiement variables a diminué. Désormais, près de $50 \%$ du soutien est soumis à des taux de paiement fixes, dont la moitié est versée indépendamment des niveaux courants. On note un abandon progressif du soutien aux produits individuels, même si la moitié environ du soutien aux producteurs va à ces produits. Le soutien versé au titre de groupes de produits a sensiblement augmenté, de même que le soutien n'imposant pas la production de produits de base. La Suisse recourt relativement peu aux transferts applicables à tous les produits dans la mesure où de nombreux paiements sont destinés au groupe des produits animaux. 
Graphique 5.2. Suisse : évolution de la composition de l'ESP par combinaison catégorie/étiquette, 1986-88 et 2005-07

Titre du transfert

2005-07

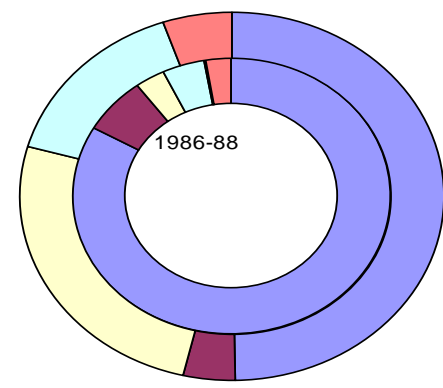

$\begin{array}{ll}\square \text { Production } & \square \text { Intrants } \\ \square \text { Superficie } & \square \text { Nombre d'animaux } \\ \square \text { Recettes/Revenu } & \square \text { Autres produits }\end{array}$

Lien à des facteurs courants

2005-07

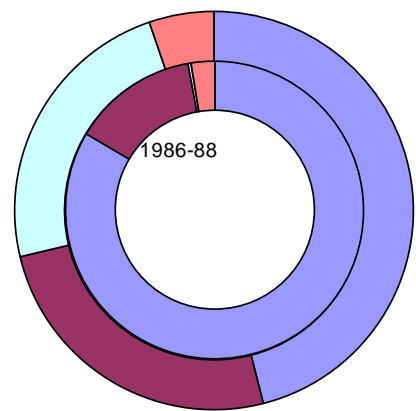

-Courant/variable $\quad$ - Courant/fixe

- Non-courant/

variable

口Non-courant/

$\square$ Autres produits (courant/n.a.)

Production requise pour obtenir le soutien

2005-07

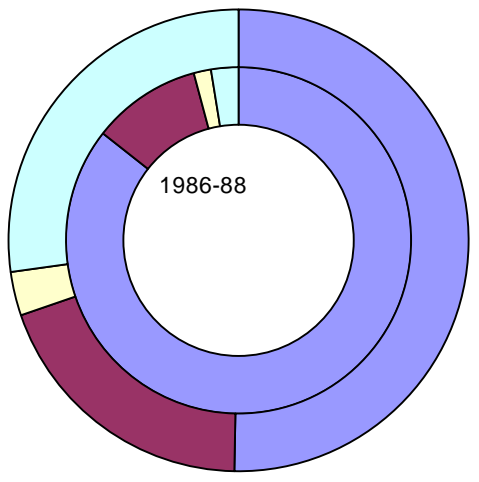

$\square$ Un seul produit

$\square$ Un groupe de produit

$\square$ Tous les produits

$\square$ Production non requise 
Graphique 5.3. UE27 et États-Unis : évolution de la composition de l'ESP par combinaison catégorie/étiquette, 1986-88 et 2005-07

Titre du transfert, $1986-88$

UE27

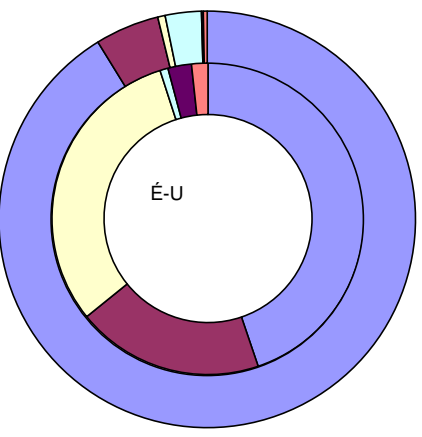

aProductio

$\square$ Superficie

口Nombre d'animaux $\mathbf{\square}$ Recettes/Revenu $\square$ Autres produits

Lien à des facteurs courants, 1986-88

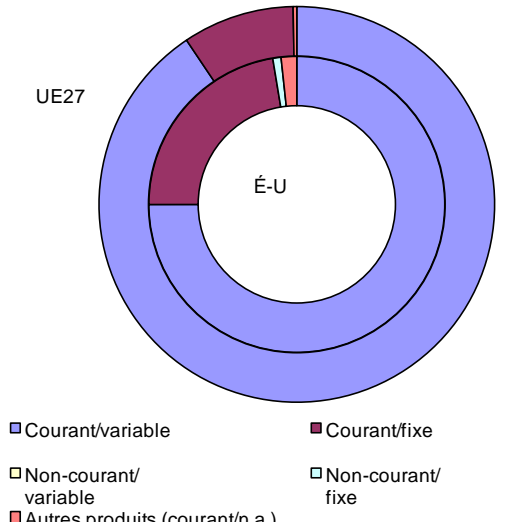

$\square$ Autres produits (courant/n.a.)

Production requise pour obtenir le soutien, 1986-88

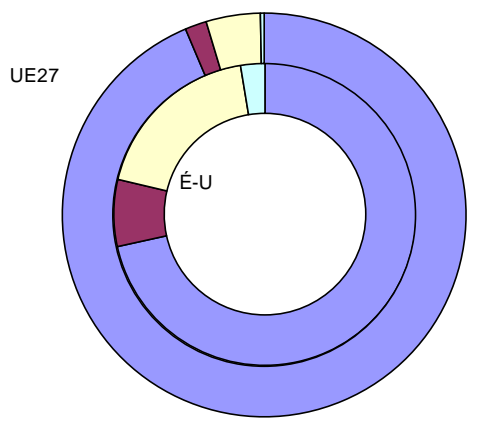

口Un seul produit 口Tous les produits
QUn groupe de produit aProduction non requise
Titre du transfert, 2005-07

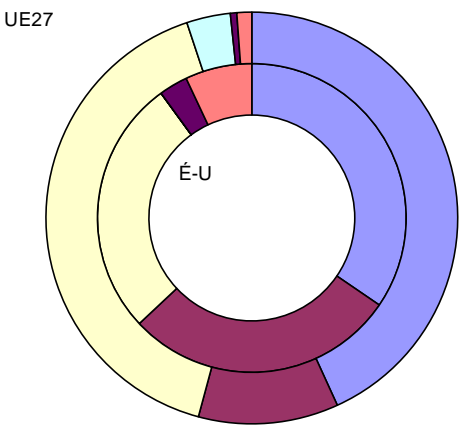

$$
\begin{array}{ll}
\square \text { Production } & \square \text { Intrants } \\
\square \text { Superficie } & \square \text { Nombre d'animaux } \\
\square \text { Recettes/Revenu } & \square \text { Autres produits }
\end{array}
$$

Lien à des facteur courants, 2005-07

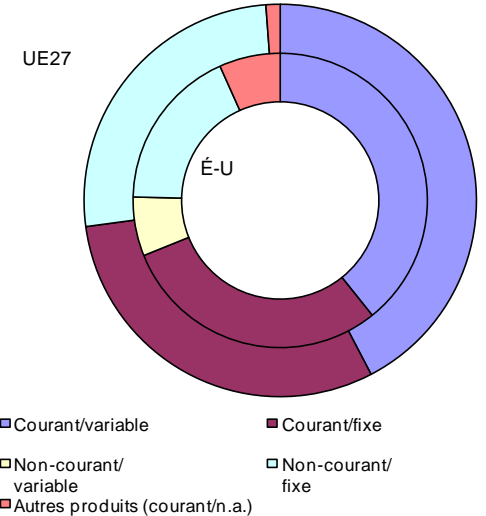

Production requise pour obtenir le soutien, 2005-07

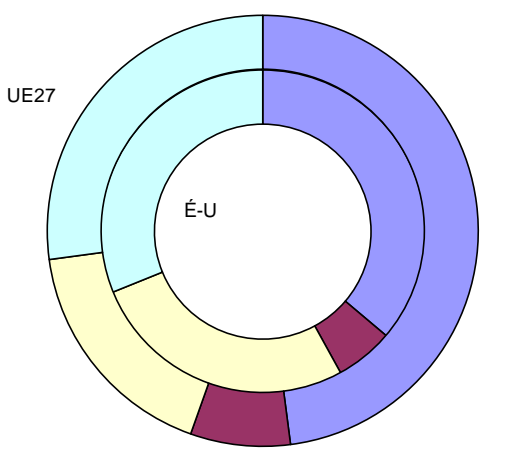

口Un seul produit

QUngroupe de produit 口Tous les produits $\square$ Production non requise 
Ce même graphique peut servir à comparer l'évolution de l'action publique de différents pays. Le graphique 5.3 met en évidence l'évolution de la composition du soutien aux producteurs dans l'UE27 (cercle extérieur) et aux États-Unis (cercle intérieur). Les cercles de la colonne de gauche correspondent à la situation en 1986-88, tandis que ceux de la colonne de droite correspondent à la situation en 2005-07. L'observation des deux colonnes indique globalement que la composition du soutien aux producteurs de l'UE27 et des États-Unis est plus proche en 2005-07 qu'elle ne l'était en 1986-88.

Pour ce qui concerne l'élément au titre duquel le soutien est accordé, on observe que la composition du soutien aux producteurs aux États-Unis n'a pas franchement évolué, mis à part la progression des transferts reposant sur des produits autres que des produits de base. Dans l'UE, à l'inverse, le soutien fondé sur la production a largement laissé la place à un soutien accordé au titre des superficies. S'agissant du lien avec des facteurs courants, on constate une évolution sensible tant dans l'UE qu'aux États-Unis, la proportion du soutien accordé en fonction du niveau effectif et assorti de taux variables ayant diminué. Par ailleurs, dans l'UE comme aux États-Unis, la proportion du soutien octroyé indépendamment des niveaux effectifs à des taux fixes a progressé. Deux distinctions doivent être faites. En premier lieu, les États-Unis accordent différents transferts au titre d'un élément non courant qui sont liés aux conditions courantes du marché, ce qui n'est pas le cas pour l'UE, où ces transferts représentent toutefois $6.5 \%$ du soutien aux producteurs en 2005-07. En second lieu, les États-Unis consacrent une proportion non négligeable des transferts à des produits autres que des produits de base, en l'occurrence $6.7 \%$ du soutien aux producteurs pour 2005-07. En ce qui concerne les produits au titre desquels un soutien est versé, les États-Unis et l'UE ont tous deux sensiblement diminué les transferts au titre de produits individuels. Tous deux ont également notablement accru le soutien accordé sans obligation de produire des produits de base, les paiements octroyés pour tous les produits étant en outre en hausse dans l'UE.

\section{Conclusions et améliorations envisageables}

Le système révisé de classification des mesures de l'ESP, qui comprend désormais douze catégories et six étiquettes, a considérablement accru le potentiel analytique des estimations du soutien mis au point par l'OCDE. Deux réserves sont toutefois à signaler. D'une part, le système de classification concerne uniquement les mesures entrant dans l'ESP, en l'occurrence celles qui prévoient des paiements aux producteurs agricoles. Les autres mesures, notamment les transferts aux services d'intérêt général, ainsi que les réglementations, taxes et redevances, n'entrent pas en ligne de compte. D'autre part, l'analyse est entièrement tributaire du travail réalisé en amont pour classer correctement les mesures dans les différentes catégories et leur accoler des étiquettes. Le Secrétariat doit en permanence veiller à ce que les mesures gouvernementales soient traitées de manière cohérente d'un pays à l'autre, en d'autres termes que les mesures présentant des caractéristiques de mise en œuvre similaires soient répertoriées et étiquetées de manière homogène.

Le rapport indique que le recours aux caractéristiques mises en lumière par les catégories et les étiquettes gagne régulièrement du terrain. La conception du système de classification a été revue de manière à refléter la complexité croissante des mesures gouvernementales, et le système semble désormais capable de prendre en compte le caractère évolutif de ces mesures. Les étiquettes sont plus particulièrement utiles pour les catégories $\mathrm{C}$, D et F. Pour ce qui concerne la catégorie F, seules les sous-catégories sont 
pertinentes - en effet, ou bien les étiquettes ne sont pas applicables, ou bien elles sont prédéfinies, ou encore toutes les mesures se voient accoler la même étiquette lorsqu'un choix est possible. Néanmoins, cet aspect ne pose pas véritablement problème compte tenu du montant des transferts impliqués.

$\mathrm{Au}$ cours de sa réflexion sur la révision du système de classification des mesures de l'ESP, le Groupe de travail ad hoc mis en place a estimé que les définitions des étiquettes étaient susceptibles d'affaiblir le système, notamment parce que l'imprécision ou le caractère générique de certaines des définitions proposées compromettait alors la puissance analytique du système (OCDE, 2007b). Avant de passer à la mise en œuvre effective du système révisé, le Secrétariat s'est donc attaché à améliorer la qualité des définitions. Les conclusions de ce rapport sur les étiquettes sont les suivantes :

- Les étiquettes définissant la relation avec les produits et si le soutien repose sur des $\mathbf{S} / \mathbf{N a} / \mathbf{R e v}$ ou Rec sont relativement claires et précises, et permettent d'affiner considérablement l'analyse et les catégories de mesures de l'ESP. La première est particulièrement utile pour analyser les évolutions dans le temps, tandis que la seconde permet de mieux faire apparaître les disparités entre pays.

- L'étiquette relative aux taux de paiement apporte également beaucoup au système de classification. Une question importante ayant suscité réflexion portait sur l'intérêt ou non d'accroître le nombre d'options proposées par cette étiquette afin d'apporter d'autres précisions sur le mode de détermination du taux de paiement.

- L'étiquette qualifiant les contraintes sur les intrants est généralement satisfaisante, mais il demeure important de continuer à établir une distinction explicite entre les termes "obligatoires" et "volontaires", tels qu'ils sont utilisés dans cette étiquette, ce qui n'est pas aussi évident qu'il peut sembler à première vue. La réflexion menée dans le cadre des travaux sur les politiques agroenvironnementales porte sur la possibilité de développer les rubriques de cette étiquette afin de cerner la nature des contraintes en question. La création de nouvelles rubriques serait extrêmement utile, mais poserait un problème pratique, à savoir jusqu'où développer les options proposées dans cette étiquette et quelle est la quantité d'informations que la base de données peut accepter.

- Le potentiel analytique de l'étiquette indiquant s'il existe une limitation de la production et/ou des paiements est relativement faible, et ce essentiellement parce qu'elle combine sous une seule possibilité (L) deux critères de mise en œuvre tout à fait différents : elle définit, d'une part, s'il est possible ou non de limiter la production et, d'autre part, si le paiement est plafonné. Dans un premier temps, il pourrait suffire de séparer ces deux critères en créant des options distinctes au sein de cette même étiquette, c'est-à-dire qu'il serait possible de déterminer si la mesure fixe une limite sur la production (PL), sur les transferts (TL), ou n'impose aucune limite (NL). Une complication pourrait toutefois survenir si une mesure plafonne à la fois la production et les paiements, auquel cas il faudrait créer deux étiquettes différentes, l'une pour la limitation des paiements et l'autre pour la limitation de la production.

- L'étiquette indiquant s'il existe des exceptions concernant les produits de base apporte relativement peu à l'analyse des politiques car elle se limite à la seule catégorie E. On pourrait envisager d'étendre l'application de cette étiquette à d'autres catégories. Elle présente par ailleurs l'inconvénient de ne donner aucune indication sur l'ampleur des interdictions de production imposées à l'agriculture. Les dispositions 
prévues peuvent varier considérablement, puisque l'interdiction peut aller de la production d'un seul produit de base jusqu'à l'ensemble des produits à l'exception d'un seul.

Outre les questions de définition, la préparation de la présente étude a mis en lumière quelques autres difficultés. En premier lieu, le système d'étiquettes n'est pas suffisamment apte à rendre compte du renforcement des mesures du fait que l'étiquette s'applique à l'ensemble de la période considérée, et non à une partie seulement. Par exemple, si une limitation des paiements ou une contrainte obligatoire sur les intrants est ajoutée à une mesure particulière déjà en vigueur, il n'existe aucun moyen de prendre cet élément en compte à moins de créer une nouvelle ligne. Pour résoudre ce problème, il faudrait utiliser un logiciel autre que Excel.

Un autre problème se pose, à savoir l'utilisation du système de classification pour répertorier des mesures en fonction de leurs objectifs. Rappelons que le système de classification des mesures de l'ESP repose sur des critères de mise en œuvre, et non sur des objectifs ou sur leur impact (sur la production, le revenu, l'environnement, etc.). Le système de classification permet toutefois d'identifier certains objectifs, par exemple des paiements agroenvironnementaux. Par ailleurs, d'autres travaux — recourant à la base de données des ESP — ont abordé la question du ciblage sur les objectifs de l'action publique (tel que l'Inventaire des mesures environnementales applicables au secteur agricole). Certes, créer une nouvelle étiquette répertoriant une série d'objectifs assignés aux mesures gouvernementales apparaîtrait techniquement faisable tout en accroissant encore la puissance analytique du système de classification et améliorant la transparence des mesures (autre caractéristique de la réforme des politiques définie par les ministres des pays de l'OCDE), mais cela entraînerait un certain nombre de difficultés. Un des problèmes fondamentaux qui se pose est que les objectifs de l'action publique sont souvent vagues et que, bien souvent, les mesures n'ont pas vocation à atteindre des objectifs explicites. Dans certains cas, les objectifs affichés peuvent différer des objectifs implicites. Se posent en outre des questions d'ordre pratique : faisabilité de l'établissement d'une liste finie d'objectifs à assigner, et attribution d'objectifs clairement définis à chaque mesure. Se pose enfin la question de savoir comment prendre en compte des objectifs multiples. En effet, les mesures mises en œuvre comportent souvent plus d'un objectif. L'une des caractéristiques du système de classification est que les étiquettes s'excluent mutuellement — en d'autres termes, seule l'une des étiquettes s'applique. Il faudrait alors accoler l'étiquette à l'objectif le plus important, ou bien faire appel à plusieurs étiquettes.

Le système de classification des mesures de l'ESP est potentiellement plus efficace pour analyser le degré précis de découplage de la production des mesures de soutien. Il permet de rendre compte des liens possibles entre critères de mise en œuvre et production. En revanche, le système est moins utile pour suivre et évaluer le degré de ciblage et d'adaptation des mesures à des résultats spécifiques, puisqu'il n'a pas été conçu dans cette optique. Il devrait néanmoins autoriser l'ajout d'étiquettes répertoriant des critères de mise en œuvre reflétant le ciblage.

L'édition 2009 du rapport sur les Politiques agricoles des pays de l'OCDE présente certaines des étiquettes comme des sous-catégories. Compte tenu de l'analyse effectuée dans le présent document, peut-on considérer qu'il s'agit là des étiquettes les plus importantes pour cerner les différences entre pays et dans le temps ? 
La valeur des transferts assortis de contraintes sur les intrants (tant obligatoires que volontaires) est indiquée pour les catégories B1, B2 et B3. Dans la mesure où ces catégories portent sur l'utilisation d'intrants, l'étiquette relative aux contraintes sur les intrants présente peut-être une utilité théorique plus grande que les autres étiquettes. Il ressort du tableau 2.3 et des tableaux annexes 3 à 5 que, pour ces trois catégories, la plupart des pays n'imposent pas de contraintes sur les intrants et que la proportion des transferts assortis de contraintes sur les intrants a relativement peu évolué au fil des ans. D'un point de vue pratique, il serait peut-être plus utile, pour mettre en évidence les modifications dans le temps et les différences entre pays pour ces trois catégories, de montrer l'importance des transferts allant à l'ensemble des produits par rapport à celle des transferts allant à des produits individuels ou des groupes de produits.

Sont indiquées pour la catégorie $\mathrm{C}$ la valeur des transferts auxquels on a attribué l'étiquette correspondant aux agrégats, soit superficies/nombre d'animaux, soit recettes/revenus, ainsi que la valeur des transferts assortis de contraintes sur les intrants. Ces deux étiquettes semblent les plus pertinentes pour cette catégorie. Comme la catégorie $\mathrm{C}$ est celle où la composition des étiquettes a le plus évolué, une étiquette, quelle qu'elle soit, fera apparaitre certaines différences entre pays et au fil du temps.

Enfin, pour la catégorie E, c'est la valeur des transferts portant l'étiquette Reposant sur des taux fixes ou variables qui est donnée. Cette étiquette a été choisie en raison de son importance théorique, c'est-à-dire que, comme les mesures donnant lieu à des transferts non liés à des paramètres courants sont assignées à cette catégorie, l'étiquette révélant le lien entre le taux de paiement et les conditions de marché courantes est utile. Du fait de son importance dans le contexte actuel, cette étiquette est utilisée comme souscatégorie dans les rapports annuels de l'OCDE sur les politiques agricoles. En pratique, cette étiquette fait ressortir les différences entre pays plutôt que les différences dans le temps. Pour étudier l'évolution temporelle à l'échelle nationale, l'étiquette indiquant si des exceptions concernant les produits de base constituent un critère d'éligibilité pourrait bien se révéler l'étiquette la plus utile.

L'intérêt du système de classification des mesures de l'ESP pour l'analyse des politiques dépend de la question posée. Le système de classification répondra bien à certaines questions, par exemple, quelle est la proportion des transferts reposant sur les superficies? Il sera en revanche moins adapté pour d'autres questions, par exemple : quels sont les types de contraintes imposées aux agriculteurs pour bénéficier du paiement au titre des superficies ? Il ne sera cependant pas en mesure de répondre à toutes les questions, par exemple, quel est le plafond des transferts qu'un agriculteur peut percevoir? Alors que le système de classification des mesures de l'ESP permet de se faire une bonne idée des principales différences caractérisant les modalités de mise en œuvre des mesures de soutien à l'agriculture dans le temps et entre pays, il est manifestement limité par la prédéfinition des catégories et étiquettes. Une analyse plus approfondie nécessitera d'examiner soigneusement ligne par ligne les mesures gouvernementales, ce qui est possible avec les informations mises à disposition du public. 


\section{Références}

OCDE (2006), Numéro spécial sur le découplage et le soutien à l'agriculture, Documents de l'OCDE, Volume 5, No. 11.

OCDE (2007a), Ciblage efficace des politiques agricoles: Bonnes pratiques pour le développement et la mise en æuvre de politiques, OCDE, Paris.

OCDE (2007b), "Report of the PSE Focus Group, 2-6 July 2007", [TAD/CA/APM/WP(2007)25].

OCDE (2008a), OECD's Producer Support Estimate and Related Indicators of Agricultural Support: Concepts, Calculations, Interpretation and Use (The PSE Manual), OCDE, Paris, www.OCDE.org/dataOCDE/18/31/41121738.pdf.

OCDE (2008b), Élaboration et mise en ouvre des politiques agricoles, Une synthèse, OCDE, Paris.

OCDE (2009a), Politiques agricoles des pays de l'OCDE: Suivi et évaluation 2009, OCDE, Paris.

OCDE (2009b), "Panorama des mesures gouvernementales ayant un lien avec le risque", [TAD/CA/APM/WP(2008)24/FINAL]. 


\section{Annexe 1. \\ Intitulés et définitions des catégories et étiquettes des mesures de l'ESP}

\section{Catégories de mesures de l'ESP}

A. Soutien au titre de la production des produits de base : transferts des consommateurs ou des contribuables aux producteurs agricoles, qui découlent des mesures accroissant les recettes unitaires (par tonne) perçues par les producteurs produisant un seul produit pendant la période en cours.

A1. Soutien des prix du marché (SPM) : transferts des consommateurs et des contribuables aux producteurs agricoles, qui découlent des mesures créant un écart entre les prix intérieurs et les prix à la frontière d'un produit agricole donné, mesuré au départ de l'exploitation.

A2. Paiements au titre de la production : transferts des contribuables aux producteurs agricoles, qui découlent des mesures fondées sur le niveau effectif de la production d'un produit agricole donné.

B. Paiements au titre de l'utilisation d'intrants : transferts des contribuables aux producteurs agricoles, qui découlent des mesures fondées sur l'utilisation d'intrants :

B1. Utilisation d'intrants variables : transferts réduisant le coût sur l'exploitation agricole d'un intrant variable donné ou d'un ensemble d'intrants variables.

B2. Formation de capital fixe : transferts réduisant pour l'exploitation, le coût des investissements dans les bâtiments agricoles, les équipements, les plantations, l'irrigation, le drainage et l'amélioration des sols.

B3. Services utilisés sur l'exploitation : transferts réduisant le coût de l'aide et de la formation dispensée aux producteurs individuels sur des questions techniques, comptables, commerciales, sanitaires et phytosanitaires.

C. Paiements au titre des S/Na/Rec/Rev courants, production requise : transferts des contribuables aux producteurs agricoles, qui découlent des mesures reposant sur le niveau effectif des superficies cultivées $(\mathrm{S})$, nombre d'animaux $(\mathrm{Na})$, recettes $(\mathrm{Rec})$ et revenus $(\mathrm{Rev})$, et pour lesquels la production est requise.

C1. Au titre des recettes/revenus courants - comprend les transferts découlant de mesures fondées sur les recettes ou les revenus.

C2. Au titre des superficies/nombres d'animaux courants - comprend les transferts découlant de mesures fondées sur les superficies/nombres d'animaux.

D. Paiements au titre des $S / \mathrm{Na} / \mathrm{Rec} / \mathrm{Rev} 1$ non courants, production requise : transferts des contribuables aux producteurs agricoles, qui découlent des mesures reposant sur les 
superficies cultivées, nombre d'animaux, recettes et revenu ne correspondant pas à la période en cours (c'est-à-dire fondées sur un niveau antérieur ou fixe), avec obligation de produire sans spécification de produit.

E. Paiements au titre des $S / N a / R e c / R e v 1$ non courants, production facultative : transferts des contribuables aux producteurs agricoles, qui découlent des mesures reposant sur les superficies cultivées, nombre d'animaux, recettes et revenus ne correspondant pas à la période en cours (c'est-à-dire fondées sur un niveau antérieur ou fixe), la production effective d'un produit donné n'étant pas obligatoire, mais facultative.

E1. Taux variables : transferts donnant lieu à des paiements dont le taux varie en fonction du niveau courant des prix des produits ou des intrants, ou de la production/des rendements et/oud es superficies.

E2. Taux fixes : transferts donnant lieu à des paiements dont le taux ne varie pas en fonction de ces paramètres.

F. Paiements selon des critères non liés à des produits de base : transferts des contribuables aux producteurs agricoles, qui découlent des mesures fondées sur :

F.1. Le retrait de ressources à long terme : transferts au titre du retrait à long terme de facteurs de production de produits de base. Les paiements répertoriés dans cette souscatégorie se distinguent de ceux imposant un retrait de ressources à court terme, qui dépendent de critères relatifs à la production des produits de base.

F.2. La production des produits particuliers autres que les produits de base : transferts alloués pour l'utilisation de ressources agricoles pour produire des biens et services spécifiques autres que des produits de base, qui ne sont pas requis par les réglementations.

F.3. D'autres critères non liés à des produits de base : transferts accordés à tous les exploitants de manière égale, par exemple un taux forfaitaire ou un paiement fixe.

G. Paiements divers : transferts des contribuables aux exploitants sur lesquels on ne dispose pas d'informations suffisantes pour pouvoir les ventiler vers les catégories appropriées.

\section{Étiquettes attachées aux mesures de l'ESP}

Avec ou sans limitation de la production effective de produits de base et/ou des paiements (avec/sans L) : définit s'il existe ou non des limitations spécifiques de la production effective des produits de base associées à une mesure prévoyant des transferts à l'agriculture et s'il existe ou non des limitations des paiements sous la forme de limitations de la superficie ou du nombre d'animaux donnant droit à ces paiements. S'applique aux catégories $\mathrm{A}-\mathrm{F}$.

Avec taux de paiement variables ou fixes (avec taux V/F) : un paiement est défini comme étant soumis à un taux variable lorsque la formule déterminant son niveau réagit à une variation du prix, du rendement, des recettes ou du revenu nets, ou du coût de production. S'applique aux catégories A - E. Pour la catégorie E, ces attributs sont présentés comme des sous-catégories supplémentaires, et non comme des étiquettes.

Avec ou sans contraintes sur les intrants (avec/sans C): définit s'il y a ou non des obligations spécifiques concernant les pratiques agricoles liées au programme en matière 
de réduction, de remplacement ou d'abandon de l'utilisation des intrants, ou des restrictions portant sur les pratiques agricoles autorisées. S'applique aux catégories A-F.

- Paiements dépendant du respect de conditions de base qui sont obligatoires (avec, obligatoires);

- Paiements exigeant des pratiques spécifiques allant au-delà des conditions de base et facultatives (avec, facultatives).

Reposant sur la superficie cultivée, le nombre d'animaux, les recettes ou le revenu $(\mathbf{S} / \mathbf{N a} / \mathbf{R e c} / \mathbf{R e v})$ : définit l'attribut particulier (à savoir la superficie cultivée, le nombre d'animaux, les recettes ou les revenus) sur lequel le paiement repose. S'applique aux catégories $\mathrm{C}-\mathrm{E}$. Pour la catégorie $\mathrm{C}$, ces attributs sont présentés comme des souscatégories supplémentaires, et non comme des étiquettes.

Reposant sur un produit individuel, un groupe de produits ou tous les produits (SC/GC/AC) : définit si le paiement est accordé pour un seul produit individuel (blé, par ex.), un groupe de produits (céréales, par exemple) ou tous les produits. S'appliquent aux catégories A - D.

Avec ou sans exceptions concernant les produits de base (avec/sans E) : définit s'il y a ou non des interdictions de production de certains produits de base comme critères d'éligibilité aux paiements versés au titre des $\mathrm{S} / \mathrm{Na} / \mathrm{Rec} / \mathrm{Rev}$ non courants. S'applique à la catégorie $\mathrm{E}$. 
Annexe 2.

Tableaux annexes 
Tableau Annexe 1. Catégorie A1 (MPS) : composition de la catégorie par pays et étiquette, 1986-2007

Part de la catégorie en pourcentage

\begin{tabular}{|c|c|c|c|c|c|c|c|c|c|c|c|c|c|c|c|c|c|c|c|c|c|c|}
\hline & 1986 & 1987 & 1988 & 1989 & 1990 & 1991 & 1992 & 1993 & 1994 & 1995 & 1996 & 1997 & 1998 & 1999 & 2000 & 2001 & 2002 & 2003 & 2004 & 2005 & 2006 & 2007 \\
\hline OCDE (USD) & 176350 & 192627 & 184505 & 170794 & 186457 & 207989 & 198393 & 193074 & 198968 & 183499 & 167805 & 152877 & 167486 & 177467 & 150599 & 125161 & 146606 & 154566 & 164100 & 146787 & 131691 & 126970 \\
\hline $\begin{array}{l}\text { Limites } \\
\text { Pas de limites }\end{array}$ & $\begin{array}{l}24 \\
76\end{array}$ & $\begin{array}{l}27 \\
73\end{array}$ & $\begin{array}{l}23 \\
77\end{array}$ & $\begin{array}{l}26 \\
74\end{array}$ & $\begin{array}{l}25 \\
75\end{array}$ & $\begin{array}{l}24 \\
76\end{array}$ & $\begin{array}{l}25 \\
75\end{array}$ & $\begin{array}{l}25 \\
75\end{array}$ & $\begin{array}{l}22 \\
78\end{array}$ & $\begin{array}{l}21 \\
79\end{array}$ & $\begin{array}{l}27 \\
73\end{array}$ & $\begin{array}{l}30 \\
70\end{array}$ & $\begin{array}{l}34 \\
66\end{array}$ & $\begin{array}{l}30 \\
70\end{array}$ & $\begin{array}{l}27 \\
73\end{array}$ & $\begin{array}{l}26 \\
74\end{array}$ & $\begin{array}{l}29 \\
71\end{array}$ & $\begin{array}{l}29 \\
71\end{array}$ & $\begin{array}{l}27 \\
73\end{array}$ & $\begin{array}{l}24 \\
76\end{array}$ & $\begin{array}{l}21 \\
79\end{array}$ & $\begin{array}{l}20 \\
80\end{array}$ \\
\hline Fixe & 2 & 2 & 1 & 2 & 2 & 2 & 1 & 1 & 1 & 1 & 1 & 1 & 1 & 1 & 1 & 1 & 2 & 2 & 2 & 2 & 3 & 2 \\
\hline Variable & 98 & 98 & 99 & 98 & 98 & 98 & 99 & 99 & 99 & 99 & 99 & 99 & 99 & 99 & 99 & 99 & 98 & 98 & 98 & 98 & 97 & 98 \\
\hline $\begin{array}{l}\text { Contraintes } \\
\text { sur les intrants }\end{array}$ & & & & & & & & & & & & & & & & & & & & & & \\
\hline $\begin{array}{l}\text { Sur res intrants } \\
\text { Un seul produit }\end{array}$ & $\begin{array}{l}\text { n.a. } \\
100\end{array}$ & $\begin{array}{l}\text { n.a. } \\
100\end{array}$ & $\begin{array}{l}\text { n.a. } \\
100\end{array}$ & $\begin{array}{l}\text { n.a. } \\
100\end{array}$ & $\begin{array}{l}\text { n.a. } \\
100 \\
\end{array}$ & $\begin{array}{l}\text { n.a. } \\
100\end{array}$ & $\begin{array}{l}\text { n.a. } \\
100\end{array}$ & $\begin{array}{l}\text { n.a. } \\
100 \\
\end{array}$ & $\begin{array}{l}\text { n.a. } \\
100\end{array}$ & $\begin{array}{l}\text { n.a. } \\
100\end{array}$ & $\begin{array}{l}\text { n.a. } \\
100 \\
\end{array}$ & $\begin{array}{l}\text { n.a. } \\
100 \\
\end{array}$ & $\begin{array}{l}\text { n.a. } \\
100\end{array}$ & $\begin{array}{l}\text { n.a. } \\
100\end{array}$ & $\begin{array}{l}\text { n.a. } \\
100\end{array}$ & $\begin{array}{l}\text { n.a. } \\
100\end{array}$ & $\begin{array}{l}\text { n.a. } \\
100\end{array}$ & $\begin{array}{l}\text { n.a. } \\
100\end{array}$ & $\begin{array}{l}\text { n.a. } \\
100\end{array}$ & $\begin{array}{l}\text { n.a. } \\
100\end{array}$ & $\begin{array}{l}\text { n.a. } \\
100\end{array}$ & $\begin{array}{l}\text { n.a. } \\
100\end{array}$ \\
\hline Australie (USD) & 766 & 515 & 178 & 831 & 1186 & 743 & 378 & 1093 & 870 & 454 & 1278 & 550 & 702 & 572 & 5 & 74 & 767 & 2 & 2 & 67 & 1 & 0 \\
\hline $\begin{array}{l}\text { Limites } \\
\text { Pas de limites }\end{array}$ & 100 & 100 & 100 & 100 & 100 & 100 & 100 & 100 & 100 & 100 & 100 & 100 & 100 & 100 & 100 & 100 & 100 & 100 & 100 & 100 & 100 & $100^{\circ}$ \\
\hline $\begin{array}{l}\text { Fixe } \\
\text { Variable }\end{array}$ & 100 & 100 & 100 & 100 & 100 & 100 & 100 & 100 & 100 & 100 & 100 & 100 & 100 & 100 & 100 & 100 & 100 & 100 & 100 & 100 & 100 & 100 \\
\hline & & & & & & & & & & & & & & & & & & & & & & \\
\hline $\begin{array}{l}\text { Sur res int } \\
\text { Un seul pit }\end{array}$ & $\begin{array}{l}\text { n.a. } \\
100\end{array}$ & $\begin{array}{l}\text { n.a. } \\
100\end{array}$ & $\begin{array}{l}\text { n.a. } \\
100\end{array}$ & $\begin{array}{l}\text { n.a. } \\
100\end{array}$ & $\begin{array}{l}\text { n.a. } \\
100\end{array}$ & $\begin{array}{l}\text { n.a. } \\
100\end{array}$ & $\begin{array}{l}\text { n.a. } \\
100\end{array}$ & $\begin{array}{l}\text { n.a. } \\
100\end{array}$ & $\begin{array}{l}\text { n.a. } \\
100\end{array}$ & $\begin{array}{l}\text { n.a. } \\
100\end{array}$ & $\begin{array}{l}\text { n.a. } \\
100\end{array}$ & $\begin{array}{l}\text { n.a. } \\
100\end{array}$ & $\begin{array}{l}\text { n.a. } \\
100\end{array}$ & $\begin{array}{l}\text { n.a. } \\
100\end{array}$ & $\begin{array}{l}\text { n.a. } \\
100\end{array}$ & $\begin{array}{l}\text { n.a. } \\
100\end{array}$ & $\begin{array}{l}\text { n.a. } \\
100\end{array}$ & $\begin{array}{l}\text { n.a. } \\
100\end{array}$ & $\begin{array}{l}\text { n.a. } \\
100\end{array}$ & $\begin{array}{l}\text { n.a. } \\
100\end{array}$ & $\begin{array}{l}\text { n.a. } \\
100\end{array}$ & $\begin{array}{l}\text { n.a. } \\
100\end{array}$ \\
\hline Canada (USD) & 3042 & 3600 & 2696 & 2744 & 4057 & 3625 & 2914 & 2804 & 2599 & 1718 & 1601 & 1924 & 2085 & 2052 & 2137 & 1624 & 2594 & 2916 & 2471 & 2940 & 3902 & 2894 \\
\hline & 48 & 59 & 68 & 61 & 43 & 60 & 60 & 59 & 66 & & & & & & 76 & & & 69 & 74 & & 75 & 76 \\
\hline Pas de limites & & & & & & & 40 & & 34 & 21 & 22 & 21 & 25 & 24 & 24 & 23 & 26 & 31 & 26 & 25 & 25 & 24 \\
\hline $\begin{array}{l}\text { Fixe } \\
\text { Vyarable }\end{array}$ & 100 & 100 & 100 & 100 & 100 & 100 & 100 & 100 & 100 & 100 & 100 & 100 & 100 & 100 & 100 & 100 & 100 & 100 & 100 & 100 & 100 & 100 \\
\hline & & & & & & & & & & & & & & & & & & & & & & \\
\hline $\begin{array}{l}\text { sur les int } \\
\text { nat }\end{array}$ & $\begin{array}{l}\text { n.a. } \\
100\end{array}$ & n.a. & n.a. & n.a. & n.a. & n.a. & n.a. & n.a. & n.a. & n.a. & n.a. & n.a. & n.a. & n.a. & n.a. & n.a. & n.a. & n.a. & n.a. & n.a. & n.a. & n.a. \\
\hline $\begin{array}{l}\text { Un seull prc } \\
\text { UE (USD) }\end{array}$ & $\begin{array}{r}100 \\
81898\end{array}$ & $\begin{array}{r}100 \\
89269\end{array}$ & $\begin{array}{r}100 \\
82243 \\
\end{array}$ & $\begin{array}{r}100 \\
60062 \\
\end{array}$ & $\begin{array}{r}100 \\
76861\end{array}$ & $\begin{array}{r}100 \\
93427\end{array}$ & $\begin{array}{r}100 \\
82564\end{array}$ & $\begin{array}{r}100 \\
70551\end{array}$ & $\begin{array}{r}100 \\
67486\end{array}$ & $\begin{array}{r}100 \\
74035\end{array}$ & $\begin{array}{r}100 \\
63860\end{array}$ & $\begin{array}{r}100 \\
59811\end{array}$ & $\begin{array}{r}100 \\
67015\end{array}$ & $\begin{array}{r}100 \\
71430\end{array}$ & $\begin{array}{r}100 \\
48586\end{array}$ & $\begin{array}{r}100 \\
41667\end{array}$ & $\begin{array}{r}100 \\
54293\end{array}$ & $\begin{array}{r}100 \\
65202\end{array}$ & $\begin{array}{r}100 \\
72819\end{array}$ & $\begin{array}{r}100 \\
59524\end{array}$ & $\begin{array}{r}100 \\
51557\end{array}$ & $\begin{array}{r}100 \\
46306\end{array}$ \\
\hline & 30 & 32 & 30 & 27 & 29 & 25 & 30 & 33 & 36 & 33 & 37 & 36 & 35 & 32 & 31 & 28 & 37 & 34 & 29 & 24 & 20 & 5 \\
\hline $\mathrm{Pa}$ & 70 & 68 & 70 & 73 & 71 & 75 & 70 & 67 & 64 & 67 & 63 & 64 & 65 & 68 & 69 & 72 & 63 & 66 & 71 & 76 & 80 & 95 \\
\hline $\begin{array}{l}\text { Fixe } \\
\text { Variable }\end{array}$ & 100 & 100 & 100 & 100 & 100 & 100 & 100 & 100 & 100 & 100 & 100 & 100 & 100 & 100 & 100 & 100 & 100 & 100 & 100 & 100 & 100 & 100 \\
\hline & & & & & & & & & & & & & & & & & & & & & & \\
\hline 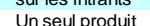 & 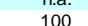 & (1.a & 100 & 10 & (1.00 & (1.60 & 100 & $\begin{array}{l}100 \\
100\end{array}$ & 100 & $\begin{array}{ll} & \\
100 & \end{array}$ & 100 & 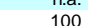 & 100 & 100 & 100 & 100 & 100 & (100 & 100 & 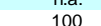 & 100 & 100 \\
\hline Island & 145 & 200 & 183 & 154 & 166 & 183 & 145 & 71 & 63 & 71 & 64 & 63 & 110 & 112 & 70 & 51 & 81 & 83 & 94 & 124 & 105 & 89 \\
\hline $\begin{array}{l}\text { Limi } \\
\text { Pas }\end{array}$ & 100 & 100 & 100 & 100 & 100 & 100 & 100 & 100 & 100 & 100 & 100 & 100 & 100 & 100 & 100 & 100 & 100 & 100 & 100 & 100 & 100 & 100 \\
\hline & & & & & & & & & & & & & & & & & & & & & & 100 \\
\hline & 100 & 100 & 100 & 100 & 100 & 100 & 100 & 100 & 100 & 100 & 100 & 100 & 100 & 100 & 100 & 100 & 100 & 100 & 100 & 100 & 100 & 100 \\
\hline $\begin{array}{l}\text { sonirumies les intrants } \\
\text { sor }\end{array}$ & & & & & & & & & & & & & & & & & & & & & & \\
\hline & & & & & & & & & & & & & & & & & & 100 & & & & 100 \\
\hline Japon (USD) & 41328 & 44382 & 47434 & 42506 & 38220 & 40951 & 47622 & 51220 & 66874 & 66209 & 52369 & 41433 & 41686 & 47016 & 47936 & 38871 & 38813 & 41547 & 42640 & 39402 & 34445 & 30003 \\
\hline & 100 & 100 & 100 & 100 & 100 & 100 & 100 & 100 & 100 & 100 & 100 & 100 & 100 & 100 & 100 & 100 & 100 & 100 & 100 & 100 & 100 & 100 \\
\hline & 100 & 100 & 100 & & & & & & & & & & & & 100 & & & & & & & \\
\hline & 100 & 100 & 100 & 100 & 100 & 100 & 100 & 100 & 100 & 100 & 100 & 100 & 100 & 100 & 100 & 100 & 100 & 100 & 100 & 100 & 100 & 100 \\
\hline sur & n.a. & n.a. & n.a. & n.a. & n.a. & n.a. & n.a. & n.a. & n.a. & n.a. & n.a. & n.a. & n.a. & n.a. & n.a. & n.a. & n.a. & n.a. & n.a. & n.a. & n.a. & n.a. \\
\hline $\begin{array}{l}\text { Un seul prodd } \\
\text { Corée (USD) }\end{array}$ & 9359 & $\begin{array}{r}100 \\
10832\end{array}$ & 15614 & $\begin{array}{r}100 \\
18111\end{array}$ & $\begin{array}{r}100 \\
18433\end{array}$ & $\begin{array}{r}100 \\
19126\end{array}$ & $\begin{array}{r}100 \\
18662\end{array}$ & $\begin{array}{r}100 \\
18533\end{array}$ & $\begin{array}{r}100 \\
20928\end{array}$ & $\begin{array}{r}100 \\
23755\end{array}$ & 21962 & 18957 & $\begin{array}{r}100 \\
11660\end{array}$ & $\begin{array}{r}100 \\
17296\end{array}$ & $\begin{array}{r}100 \\
18376\end{array}$ & $\begin{array}{r}100 \\
14892\end{array}$ & $\begin{array}{l}100 \\
16186\end{array}$ & $\begin{array}{l}100 \\
15751\end{array}$ & $\begin{array}{l}100 \\
19125\end{array}$ & $\begin{array}{r}100 \\
20888\end{array}$ & $\begin{array}{r}100 \\
22847\end{array}$ & $\begin{array}{r}100 \\
23151\end{array}$ \\
\hline & 100 & 100 & & & 100 & 100 & 100 & 100 & & & & & & & 100 & & 100 & & $100+2>$ & 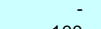 & 10 & 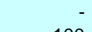 \\
\hline limites & 100 & 100 & 100 & 100 & 100 & 100 & 100 & 100 & 100 & 100 & 100 & & & 100 & 100 & 100 & 100 & 100 & 100 & 100 & 100 & 100 \\
\hline & 100 & 100 & 100 & 100 & 100 & 100 & 100 & 100 & 100 & 100 & 100 & 100 & 100 & 100 & 100 & 100 & 100 & 100 & 100 & 100 & 100 & 100 \\
\hline $\begin{array}{l}\text { Contrain } \\
\text { sur les in }\end{array}$ & n.a. & & & & & & & & & & & & & & n.a. & & & & n.a. & & n.a. & n.a. \\
\hline Un seul produit & 100 & 100 & 100 & 100 & 100 & 100 & 100 & 100 & 100 & 100 & 100 & 100 & 100 & 100 & 100 & 100 & 100 & 100 & 100 & 100 & 100 & 100 \\
\hline
\end{tabular}


Tableau Annexe 1. Catégorie A1 (MPS) : composition de la catégorie par pays et étiquettes, 1986-2007 (suite)

Part de la catégorie en pourcentage

\begin{tabular}{|c|c|c|c|c|c|c|c|c|c|c|c|c|c|c|c|c|c|c|c|c|c|c|}
\hline & 1986 & 1987 & 1988 & 1989 & 1990 & 1991 & 1992 & 1993 & 1994 & 1995 & 1996 & 1997 & 1998 & 1999 & 2000 & 2001 & 2002 & 2003 & 2004 & 2005 & 2006 & 2007 \\
\hline Mexique (USD) & (888) & (398) & (1697) & 964 & 1751 & 5996 & 6873 & 7728 & 3868 & (2 807) & $\begin{array}{l}(434) \\
\end{array}$ & 2666 & 3702 & 3533 & 5177 & 3708 & 6635 & 3297 & 1135 & 1392 & 2228 & 1654 \\
\hline Limites & (0) & (0) & (0) & & & & & & & (0) & (0) & & & & & & & & & 0 & & 4 \\
\hline Pas de limites & 100 & 100 & 100 & 100 & 100 & 100 & 100 & 100 & 100 & 100 & 100 & 100 & 100 & 100 & 100 & 100 & 100 & 100 & 100 & 100 & 100 & 100 \\
\hline Fixe & (0) & (0) & (0) & & & & & & & (0) & (0) & & & & & & & & & & & \\
\hline Variable & 100 & 100 & 100 & 100 & 100 & 100 & 100 & 100 & 100 & 100 & 100 & 100 & 100 & 100 & 100 & 100 & 100 & 100 & 100 & 100 & 100 & 100 \\
\hline \multicolumn{23}{|l|}{ Contraintes } \\
\hline sur les intrants & n.a. & n.a. & n.a. & n.a. & n.a. & n.a. & n.a. & n.a. & n.a. & n.a. & n.a. & n.a. & n.a. & n.a. & n.a. & n.a. & n.a. & n.a. & n.a. & n.a. & n.a. & n.a. \\
\hline & 68 & 70 & 48 & 41 & 38 & 43 & 25 & 17 & 34 & 55 & 29 & 36 & 23 & 24 & 0 & 18 & 4 & 42 & 35 & 70 & 55 & $\frac{100}{37}$ \\
\hline \multicolumn{23}{|l|}{$\begin{array}{l}\text { Nelle-Zélande (USD) } \\
\text { Limites }\end{array}$} \\
\hline Pas de limites & 100 & 100 & 100 & 100 & 100 & 100 & 100 & 100 & 100 & 100 & 100 & 100 & 100 & 100 & 100 & 100 & 100 & 100 & 100 & 100 & 100 & 100 \\
\hline Fixe & & & & & & & & & & & & & & & & & & & & & & \\
\hline Variable & 100 & 100 & 100 & 100 & 100 & 100 & 100 & 100 & 100 & 100 & 100 & 100 & 100 & 100 & 100 & 100 & 100 & 100 & 100 & 100 & 100 & 100 \\
\hline \multicolumn{23}{|l|}{ Contraintes } \\
\hline $\begin{array}{l}\text { sur les intrants } \\
\text { Un seul produit }\end{array}$ & $\begin{array}{l}\text { n.a. } \\
100\end{array}$ & $\begin{array}{l}\text { n.a. } \\
100\end{array}$ & $\begin{array}{l}\text { n.a. } \\
100\end{array}$ & $\begin{array}{l}\text { n.a. } \\
100\end{array}$ & n.a. & $\begin{array}{l}\text { n.a. } \\
100\end{array}$ & n.a. & n.a. & n.a. & n.a. & $\begin{array}{l}\text { n.a. } \\
100\end{array}$ & n.a. & n.a. & n.a. & $\begin{array}{l}\text { n.a. } \\
100\end{array}$ & $\begin{array}{l}\text { n.a. } \\
100\end{array}$ & $\begin{array}{l}\text { n.a. } \\
100\end{array}$ & $\begin{array}{l}\text { n.a. } \\
100\end{array}$ & $\begin{array}{l}\text { n.a. } \\
100\end{array}$ & n.a. & $\begin{array}{l}\text { n.a. } \\
100\end{array}$ & $\begin{array}{l}\text { n.a. } \\
100\end{array}$ \\
\hline Norvège (USD) & 1238 & 1478 & 1325 & 1283 & 1781 & 1703 & 1478 & 1336 & 1276 & 1263 & 1366 & 1281 & 1319 & 1207 & 843 & 878 & 1347 & 1441 & 1334 & 1378 & 1326 & 912 \\
\hline Limites & 16 & 17 & 15 & 14 & 26 & 24 & 30 & 28 & 32 & 25 & 34 & 34 & 34 & 32 & 29 & 27 & 34 & 29 & 25 & 27 & 26 & 0 \\
\hline Pas de limites & 84 & 83 & 85 & 86 & 74 & 76 & 70 & 72 & 68 & 75 & 66 & 66 & 66 & 68 & 71 & 73 & 66 & 71 & 75 & 73 & 74 & 100 \\
\hline Fixe & & & & & & & & & & & & & & & & & & & & & & \\
\hline Variable & 100 & 100 & 100 & 100 & 100 & 100 & 100 & 100 & 100 & 100 & 100 & 100 & 100 & 100 & 100 & 100 & 100 & 100 & 100 & 100 & 100 & 100 \\
\hline \multicolumn{23}{|l|}{ Contraintes } \\
\hline sur les intran & n.a. & n.a. & n.a. & n.a. & n.a. & n.a. & n.a. & n.a. & n.a. & n.a. & n.a. & n.a. & n.a. & n.a. & n.a. & n.a. & n.a. & n.a. & n.a. & n.a. & n.a. & \\
\hline & 100 & 100 & 100 & 100 & 100 & 100 & 100 & 100 & 100 & 100 & 100 & 100 & 100 & 100 & 100 & 100 & 100 & 100 & 100 & 100 & 100 & 100 \\
\hline Suisse (USD) & 3761 & 4678 & 4927 & 3863 & 4975 & 4856 & 4029 & 4180 & 4386 & 4120 & 3900 & 3200 & 3334 & 3183 & 2541 & 2307 & 2807 & 2929 & 3119 & 2963 & 2261 & 1414 \\
\hline Limites & 38 & 37 & 36 & 31 & 34 & 32 & 35 & 36 & 36 & 33 & 44 & 44 & 43 & 43 & 25 & 28 & 31 & 26 & 21 & 24 & 17 & 0 \\
\hline Pas de limites & 62 & 63 & 64 & 69 & 66 & 68 & 65 & 64 & 64 & 67 & 56 & 56 & 57 & 57 & 75 & 72 & 69 & 74 & 79 & 76 & 83 & \\
\hline \multicolumn{23}{|l|}{ Fixe } \\
\hline Variable & 100 & 100 & 100 & 100 & 100 & 100 & 100 & 100 & 100 & 100 & 100 & 100 & 100 & 100 & 100 & 100 & 100 & 100 & 100 & 100 & 100 & 100 \\
\hline \multicolumn{23}{|l|}{ Contraintes } \\
\hline sur les intrants & n.a. & n.a. & n.a. & n.a. & n.a. & n.a. & n.a. & n.a. & n.a. & n.a. & n.a. & n.a. & n.a. & n.a. & n.a. & n.a. & n.a. & n.a. & n.a. & n.a. & n.a. & \\
\hline Un seul p & 100 & 100 & 100 & 100 & 100 & 100 & 100 & 100 & 100 & 100 & 100 & 100 & 100 & 100 & 100 & 100 & 100 & 100 & 100 & 100 & 100 & 100 \\
\hline Turquie (USD) & 2051 & 2980 & 1637 & 2867 & 5309 & 7187 & 6382 & 5945 & 1795 & 2071 & 3354 & 6165 & 8416 & 5496 & 5360 & (15) & 4339 & 8684 & 8987 & 9620 & 6786 & 8676 \\
\hline $\begin{array}{l}\text { Limites } \\
\text { Pas de limites }\end{array}$ & 100 & 100 & 100 & 100 & 100 & 100 & 100 & 100 & 100 & 100 & 100 & 100 & 100 & 100 & 100 & $\begin{array}{c}100 \\
(0)\end{array}$ & 100 & 100 & 100 & 100 & 100 & 100 \\
\hline Fixe & & & & & & & & & & & & & & & & (0) & & & & & & \\
\hline \multirow{2}{*}{\multicolumn{23}{|c|}{ Contraintes }} \\
\hline & & & & & & & & & & & & & & & & & & & & & & \\
\hline sur les intrants & n.a. & $n$ & n.a. & n.a. & n.a. & n.a. & n.a. & n.a. & n.a. & n.a. & n.a. & n.a. & n.a. & n.a. & $\mathrm{n}$ & n.a. & n.a. & n.a. & n.a. & n.a. & n.a. & n.a. \\
\hline Un seul produit & 100 & 100 & 100 & 100 & 100 & 100 & 100 & 100 & 100 & 100 & 100 & 100 & 100 & 100 & 100 & 100 & 100 & 100 & 100 & 100 & 100 & 100 \\
\hline Etats-Unis (USD) & 12426 & 16476 & 12019 & 21629 & 14460 & 15372 & 15069 & 16747 & 14420 & 9278 & 14375 & 13977 & 21320 & 21144 & 17431 & 18163 & 15096 & 10332 & 12340 & 8421 & 6178 & 11834 \\
\hline $\begin{array}{l}\text { Limites } \\
\text { Pas de limites }\end{array}$ & 100 & 100 & 100 & 100 & 100 & 100 & 100 & 100 & 100 & 100 & 100 & 100 & 100 & 100 & 100 & 100 & 100 & 100 & 100 & 100 & 100 & 100 \\
\hline \multicolumn{23}{|l|}{$\begin{array}{l}\text { Fixe } \\
\text { Fixe }\end{array}$} \\
\hline \multirow{2}{*}{\multicolumn{22}{|c|}{$\begin{array}{l}\text { Variable } \\
\text { Contraintes }\end{array}$}} & 100 \\
\hline & & & & & & & & & & & & & & & & & & & & & & \\
\hline sur les intrants & n.a. & n.a. & n.a. & n.a. & & n.a. & & n.a. & n.a. & n.a. & n.a. & n.a. & n.a. & n.a. & n.a. & n.a. & n.a. & n.a. & n.a. & n.a. & n.a. & n.a. \\
\hline Un seul produit & 100 & 100 & 100 & 100 & 100 & 100 & 100 & 100 & 100 & 100 & 100 & 100 & 100 & 100 & 100 & 100 & 100 & 100 & 100 & 100 & 100 & 100 \\
\hline
\end{tabular}


Tableau Annexe 2. Catégorie A2 (PO) : composition de la catégorie par pays et étiquette, 1986-2007

Part de la catégorie en pourcentage

\begin{tabular}{|c|c|c|c|c|c|c|c|c|c|c|c|c|c|c|c|c|c|c|c|c|c|c|}
\hline & 1986 & 1987 & 1988 & 1989 & 1990 & 1991 & 1992 & 1993 & 1994 & 1995 & 1996 & 1997 & 1998 & 1999 & 2000 & 2001 & 2002 & 2003 & 2004 & 2005 & 2006 & 2007 \\
\hline $\begin{array}{l}\text { OCDE (USD) } \\
\text { Limites }\end{array}$ & 12576 & $\begin{array}{r}11634 \\
74\end{array}$ & $\begin{array}{l}12454 \\
67\end{array}$ & 12323 & 14352 & 10834 & 8436 & 6889 & 5548 & 7653 & 6808 & 6590 & 10302 & 17504 & 18182 & 17083 & 9477 & 11067 & 16252 & 16385 & 7829 & 5044 \\
\hline $\begin{array}{l}\text { Limites } \\
\text { Pas de limites }\end{array}$ & 21 & $\begin{array}{l}74 \\
26\end{array}$ & 33 & $\begin{array}{l}68 \\
32\end{array}$ & $\begin{array}{l}71 \\
29\end{array}$ & $\begin{array}{l}77 \\
23\end{array}$ & $\begin{array}{l}87 \\
13\end{array}$ & $\begin{array}{l}86 \\
14\end{array}$ & $\begin{array}{l}76 \\
24\end{array}$ & $\begin{array}{l}85 \\
15\end{array}$ & $\begin{array}{l}87 \\
13\end{array}$ & $\begin{array}{l}88 \\
12\end{array}$ & $\begin{array}{l}87 \\
13\end{array}$ & $\begin{array}{l}84 \\
16\end{array}$ & $\begin{array}{l}72 \\
28\end{array}$ & $\begin{array}{l}72 \\
28\end{array}$ & $\begin{array}{l}63 \\
37\end{array}$ & $\begin{array}{l}74 \\
26\end{array}$ & $\begin{array}{l}77 \\
23\end{array}$ & $\begin{array}{l}80 \\
20\end{array}$ & $\begin{array}{l}59 \\
41\end{array}$ & $\begin{array}{l}73 \\
27\end{array}$ \\
\hline $\begin{array}{l}\text { Fixe } \\
\text { Variable }\end{array}$ & $\begin{array}{l}32 \\
68\end{array}$ & $\begin{array}{l}39 \\
61\end{array}$ & $\begin{array}{l}47 \\
53\end{array}$ & $\begin{array}{l}50 \\
50\end{array}$ & $\begin{array}{l}54 \\
46\end{array}$ & $\begin{array}{l}52 \\
48\end{array}$ & $\begin{array}{l}72 \\
28\end{array}$ & $\begin{array}{l}65 \\
35\end{array}$ & $\begin{array}{l}57 \\
43\end{array}$ & $\begin{array}{l}69 \\
31\end{array}$ & $\begin{array}{l}79 \\
21\end{array}$ & $\begin{array}{l}72 \\
28\end{array}$ & $\begin{array}{l}46 \\
54\end{array}$ & $\begin{array}{l}30 \\
70\end{array}$ & $\begin{array}{l}33 \\
67\end{array}$ & $\begin{array}{l}33 \\
67\end{array}$ & $\begin{array}{l}61 \\
39\end{array}$ & $\begin{array}{l}59 \\
41\end{array}$ & $\begin{array}{r}55 \\
45\end{array}$ & $\begin{array}{r}55 \\
45\end{array}$ & $\begin{array}{l}60 \\
40\end{array}$ & $\begin{array}{l}79 \\
21\end{array}$ \\
\hline $\begin{array}{l}\text { Avec C. (obligatoire) } \\
\text { Anter }\end{array}$ & 38 & 22 & 11 & 6 & 5 & 2 & 5 & 5 & 6 & 1 & 1 & 5 & 41 & 60 & 56 & 54 & 19 & 26 & 48 & 49 & 36 & 12 \\
\hline $\begin{array}{l}\text { Avec c. (volontaire) } \\
\text { Sans contrainte }\end{array}$ & $\begin{array}{r}0 \\
61\end{array}$ & $\begin{array}{r}0 \\
78\end{array}$ & $\begin{array}{r}0 \\
89\end{array}$ & $\begin{array}{r}0 \\
94\end{array}$ & $\begin{array}{r}0 \\
95\end{array}$ & $\begin{array}{r}0 \\
98\end{array}$ & $\begin{array}{r}0 \\
95\end{array}$ & $\begin{array}{r}1 \\
94\end{array}$ & $\begin{array}{r}1 \\
94\end{array}$ & $\begin{array}{r}1 \\
98\end{array}$ & $\begin{array}{r}1 \\
98\end{array}$ & $\begin{array}{r}1 \\
94\end{array}$ & $\begin{array}{r}0 \\
58 \\
58\end{array}-10$ & $\begin{array}{r}0 \\
40\end{array}$ & $\begin{array}{r}0 \\
44\end{array}$ & $\begin{array}{r}0 \\
45\end{array}$ & $\begin{array}{r}0 \\
81\end{array}$ & $\begin{array}{r}0 \\
74\end{array}$ & $\begin{array}{r}0 \\
52\end{array}$ & $\begin{array}{r}0 \\
50\end{array}$ & $\begin{array}{c}0 \\
64\end{array}$ & ${ }_{76}^{12}$ \\
\hline $\begin{array}{l}\text { Un seul produit } \\
\text { Australi (IISD) }\end{array}$ & & & & & 100 & 100 & 100 & & & $\begin{array}{r}100 \\
100-2 x-10\end{array}$ & 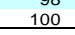 & 100 & $\begin{array}{r}100 \\
\end{array}$ & 100 & 100 & 100 & 100 & $\begin{array}{l}100 \\
\end{array}$ & 100 & $\begin{array}{r}50 \\
100 \\
\end{array}$ & $\begin{array}{r}64 \\
100\end{array}$ & $\begin{array}{l}16 \\
100 \\
\end{array}$ \\
\hline \multicolumn{23}{|l|}{$\begin{array}{l}\text { Australie (USD) } \\
\text { Limites }\end{array}$} \\
\hline \multicolumn{23}{|l|}{$\begin{array}{l}\text { Pas de limites } \\
\text { Fixe }\end{array}$} \\
\hline \multicolumn{23}{|l|}{$\begin{array}{l}\text { arabale } \\
\text { Avec (obligatoire) } \\
\text { Avec c. (volontaire) } \\
\text { Sans contrainte }\end{array}$} \\
\hline $\begin{array}{l}\text { Un seul produit } \\
\text { Canada (USD) }\end{array}$ & 365 & 303 & 511 & 540 & 293 & 383 & 283 & 181 & 169 & 151 & 115 & 103 & 81 & 62 & 42 & 19 & - & - & - & - & - & \\
\hline $\begin{array}{l}\text { Limites } \\
\text { Pas de limites }\end{array}$ & $\begin{array}{l}56 \\
44\end{array}$ & $\begin{array}{l}72 \\
28\end{array}$ & $\begin{array}{l}46 \\
54\end{array}$ & $\begin{array}{l}42 \\
58\end{array}$ & $\begin{array}{l}77 \\
23\end{array}$ & $\begin{array}{l}58 \\
42\end{array}$ & $\begin{array}{l}65 \\
35 \\
35\end{array}$ & 97 & $\begin{array}{r}100 \\
0\end{array}$ & 100 & 100 & 100 & 100 & 100 & 100 & 100 & & & & & & \\
\hline $\begin{array}{l}\text { Fixe } \\
\text { Fixe }\end{array}$ & 0 & in & 1 & 100 & 100 & 100 & 100 & $0^{-1}$ & 100 & $100^{\circ}$ & 100 & 100 & 100 & 100 & 100 & 100 & & & & & & \\
\hline $\begin{array}{l}\text { Avecc. (obligatire) } \\
\text { Avecc c. (volontaire) }\end{array}$ & & & & & & & & & & & & & & & & & & & & & & \\
\hline Sans contrainte & 100 & 100 & 100 & 100 & 100 & 100 & 100 & 100 & 100 & 100 & 100 & 100 & 100 & 100 & 100 & 100 & & & & & & \\
\hline $\begin{array}{l}\text { Un seul produit } \\
\text { UE (USD) }\end{array}$ & $\begin{array}{r}100 \\
4362\end{array}$ & $\begin{array}{r}100 \\
5462\end{array}$ & $\begin{array}{r}100 \\
6440\end{array}$ & $\begin{array}{r}100 \\
7076\end{array}$ & $\begin{array}{r}100 \\
8952\end{array}$ & $\begin{array}{r}100 \\
7485\end{array}$ & $\begin{array}{r}100 \\
4989 \\
4989\end{array}$ & $\begin{array}{r}100 \\
3063\end{array}$ & $\begin{array}{r}100 \\
2210\end{array}$ & $\begin{array}{r}100 \\
4390\end{array}$ & $\begin{array}{r}100 \\
4668\end{array}$ & $\begin{array}{r}100 \\
3977\end{array}$ & $\begin{array}{r}100 \\
4008\end{array}$ & $\begin{array}{r}100 \\
4162\end{array}$ & $\begin{array}{r}100 \\
4330\end{array}$ & $\begin{array}{r}100 \\
3662\end{array}$ & 3794 & 4349 & 6831 & 6350 & 1751 & \\
\hline 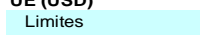 & $\begin{array}{r}4522 \\
81\end{array}$ & $\begin{array}{r}302 \\
78\end{array}$ & $\begin{array}{r}6440 \\
79\end{array}$ & 79 & $\begin{array}{r}852 \\
80\end{array}$ & $\begin{array}{r}775 \\
77\end{array}$ & $\begin{aligned} 4969 \\
94\end{aligned}$ & $\begin{array}{r}953 \\
95\end{array}$ & $\begin{array}{r}210 \\
88\end{array}$ & $\begin{array}{r}4390 \\
93\end{array}$ & $\begin{array}{r}4008 \\
92\end{array}$ & $\begin{array}{r}9211 \\
92\end{array}$ & $\begin{array}{r}4008 \\
91\end{array}$ & $\begin{array}{r}4102 \\
92\end{array}$ & $\begin{array}{r}430 \\
86\end{array}$ & $\begin{array}{r}502 \\
91\end{array}$ & $\begin{array}{r}3.74 \\
94\end{array}$ & $\begin{array}{r}4349 \\
97\end{array}$ & 97 & $\begin{array}{r}-3500 \\
99\end{array}$ & 97 & $\begin{array}{r}828 \\
96\end{array}$ \\
\hline Pas de limites & 19 & 22 & 21 & 21 & 20 & ${ }^{23}$ & 6 & 5 & 12 & 7 & 8 & 8 & 9 & 8 & 14 & 9 & 6 & 3 & 3 & 1 & 3 & \\
\hline $\begin{array}{l}\text { Fixe } \\
\text { Variable }\end{array}$ & $\begin{array}{l}41 \\
59\end{array}$ & $\begin{array}{l}38 \\
62\end{array}$ & $\begin{array}{l}51 \\
49\end{array}$ & $\begin{array}{l}466 \\
54\end{array}$ & $\begin{array}{l}499 \\
51\end{array}$ & $\begin{array}{l}54 \\
46\end{array}$ & $\begin{array}{r}94 \\
6\end{array}$ & $\begin{array}{r}94 \\
6\end{array}$ & $\begin{array}{r}92 \\
8\end{array}$ & $\begin{array}{r}94 \\
6\end{array}$ & $\begin{array}{r}92 \\
8\end{array}$ & $\begin{array}{r}92 \\
8\end{array}$ & $\begin{array}{r}93 \\
7\end{array}$ & $\begin{array}{c}93 \\
7\end{array}$ & $\begin{array}{l}83 \\
17\end{array}$ & $\begin{array}{l}89 \\
11\end{array}$ & $\begin{array}{r}92 \\
8\end{array}$ & $\begin{array}{c}94 \\
6\end{array}$ & $\begin{array}{c}97 \\
3\end{array}$ & $\begin{array}{c}98 \\
2\end{array}$ & $\begin{array}{r}91 \\
9\end{array}$ & $\begin{array}{l}100 \\
(0)\end{array}$ \\
\hline \multicolumn{23}{|l|}{$\begin{array}{l}\text { Aarlabele (obligatioire) } \\
\text { Avec c. (volontaire) }\end{array}$} \\
\hline Sans contrainte & 100 & 100 & 100 & 100 & 100 & 100 & 100 & 100 & 100 & 100 & 100 & 100 & 100 & 100 & 100 & 100 & 100 & 100 & 71 & 69 & 48 & 82 \\
\hline Un seul produ & 100 & 100 & 100 & 100 & 100 & 100 & 100 & 100 & 100 & 100 & 100 & 100 & 100 & 100 & 100 & 100 & 100 & $\frac{100}{55^{2}}$ & 100 & 100 & 100 & 100 \\
\hline $\begin{array}{l}\text { Islande USD) } \\
\text { Limites }\end{array}$ & & & 5 & 4 & 4 & 4 & $\begin{array}{l}34 \\
88\end{array}$ & $\begin{array}{l}65 \\
96\end{array}$ & $\begin{array}{l}57 \\
99\end{array}$ & $\begin{array}{r}63 \\
100\end{array}$ & $\begin{array}{r}39 \\
100\end{array}$ & $\begin{array}{r}38 \\
100\end{array}$ & $\begin{array}{r}41 \\
100\end{array}$ & $\begin{array}{r}42 \\
100\end{array}$ & $\begin{array}{r}41 \\
100\end{array}$ & $\begin{array}{r}36 \\
100\end{array}$ & $\begin{array}{l}44 \\
95\end{array}$ & $\begin{array}{l}53 \\
96\end{array}$ & $\begin{array}{l}60 \\
95\end{array}$ & $\begin{array}{l}69 \\
95\end{array}$ & $\begin{array}{l}63 \\
96\end{array}$ & $\begin{array}{l}66 \\
95 \\
95\end{array}$ \\
\hline Pas de limites & & & 100 & 100 & 100 & 100 & 12 & 4 & 1 & & 0 & & & & & & 5 & 4 & 5 & 5 & 4 & 5 \\
\hline $\begin{array}{l}\text { Fixe } \\
\text { Variable }\end{array}$ & & & 100 & 100 & 100 & 100 & 100 & 100 & 100 & 100 & 100 & 100 & 100 & 100 & 100 & 100 & 100 & 100 & 100 & 100 & 100 & 100 \\
\hline \multicolumn{23}{|l|}{$\begin{array}{l}\text { Avec c. (obligatoire) } \\
\text { Avec c. (volontaire) }\end{array}$} \\
\hline Sans contrainte & & & 100 & 100 & 100 & 100 & 100 & 100 & 100 & 100 & 100 & 100 & 100 & 100 & 100 & 100 & 100 & 100 & 100 & 100 & 100 & 100 \\
\hline $\begin{array}{l}\text { Unseuproud } \\
\end{array}$ & & & 2000 & 100 & 100 & $\begin{array}{r}100 \\
1454\end{array}$ & 100 & 100 & 100 & 100 & 100 & 100 & 100 & 100 & 100 & 100 & 100 & 100 & 100 & 100 & 100 & 100 \\
\hline Japon (USD) & 1253 & 1357 & 2006 & 1408 & 1414 & 1454 & 1633 & 1987 & 2028 & 2212 & 1202 & 1433 & 1240 & 1657 & 2436 & 2389 & 2248 & 2496 & 2414 & 2089 & 1999 & 1308 \\
\hline $\begin{array}{l}\text { Limites } \\
\text { Pas de limites }\end{array}$ & $\begin{array}{l}78 \\
22\end{array}$ & $\begin{array}{l}74 \\
26\end{array}$ & $\begin{array}{l}56 \\
44\end{array}$ & $\begin{array}{l}83 \\
17\end{array}$ & $\begin{array}{l}84 \\
16\end{array}$ & $\begin{array}{l}86 \\
14\end{array}$ & $\begin{array}{l}79 \\
21\end{array}$ & $\begin{array}{l}74 \\
26\end{array}$ & $\begin{array}{l}58 \\
42\end{array}$ & $\begin{array}{l}70 \\
30\end{array}$ & $\begin{array}{l}82 \\
18\end{array}$ & $\begin{array}{l}88 \\
12\end{array}$ & $\begin{array}{l}77 \\
23\end{array}$ & $\begin{array}{l}72 \\
28\end{array}$ & $\begin{array}{l}53 \\
47\end{array}$ & $\begin{array}{l}47 \\
53\end{array}$ & $\begin{array}{l}43 \\
57\end{array}$ & $\begin{array}{l}36 \\
64\end{array}$ & $\begin{array}{l}37 \\
63\end{array}$ & $\begin{array}{l}35 \\
65\end{array}$ & $\begin{array}{l}38 \\
62\end{array}$ & $\begin{array}{l}42 \\
58\end{array}$ \\
\hline Fixe & 37 & 36 & 23 & 28 & 21 & 21 & $18 \mathrm{~T}$ & 14 & 15 & 16 & 27 & 21 & 24 & 22 & 47 & 46 & 52 & 58 & 60 & 67 & 65 & 60 \\
\hline Variable & 63 & 64 & 77 & 72 & 79 & 79 & 82 & 86 & 85 & 84 & 73 & 79 & 76 & 78 & 53 & 54 & 48 & 42 & 40 & 33 & 35 & 40 \\
\hline \multicolumn{23}{|l|}{$\begin{array}{l}\text { Avec c. (obligatoire) } \\
\text { Avec c. (volontaire) }\end{array}$} \\
\hline 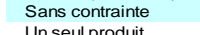 & 100 & 100 & 100 & 100 & 100 & 100 & 100 & 100 & 100 & 100 & 100 & 100 & 100 & 100 & 100 & 100 & 100 & 100 & 100 & 100 & 100 & 56 \\
\hline \multicolumn{23}{|l|}{$\begin{array}{l}\text { Un sueul produit } \\
\text { Corée (USD) }\end{array}$} \\
\hline $\begin{array}{l}\text { Limites } \\
\text { Pas de limites }\end{array}$ & & & & 100 & 100 & & & & & & & & & & & & & & & & & \\
\hline $\begin{array}{l}\text { Pas de limites } \\
\text { Fixe }\end{array}$ & & & & $\begin{array}{l}\begin{array}{l}100 \\
100\end{array}\end{array}$ & $\begin{array}{l}\begin{array}{l}100 \\
100\end{array}\end{array}$ & $\begin{array}{l}\begin{array}{l}100 \\
100\end{array}\end{array}$ & $\begin{array}{l}100 \\
100\end{array}$ & & & & & & & & & & & & & & & \\
\hline \multicolumn{23}{|l|}{$\begin{array}{l}\text { galaire) } \\
\text { notaire) }\end{array}$} \\
\hline $\begin{array}{l}\text { Avecc c. (vo } \\
\text { Sans contr }\end{array}$ & & & & 100 & 100 & 100 & 100 & & & & & & & & & & & & & & & \\
\hline Un seul prod & & & & & & & & & & & & & & & & & & & & & & \\
\hline
\end{tabular}

ANALYSE DE LA COMPOSITION DU SOUTIEN AUX PRODUCTEURS : NOUVEAUX OUTILS ET MÉTHODES ๑ OECD 2010 
Tableau Annexe 2. Catégorie A2 (PO) : composition de la catégorie par pays et étiquette, 1986-2007 (suite) Part de la catégorie en pourcentage

\begin{tabular}{|c|c|c|c|c|c|c|c|c|c|c|c|c|c|c|c|c|c|c|c|c|c|c|}
\hline & 1986 & 1987 & 1988 & 1989 & 1990 & 1991 & 1992 & 1993 & 1994 & 1995 & 1996 & 1997 & 1998 & 1999 & 2000 & 2001 & 2002 & 2003 & 2004 & 2005 & 2006 & $\frac{2007}{170}$ \\
\hline Mexique (USD) & 2 & 1 & 1 & 2 & 17 & 39 & 46 & 69 & 58 & 29 & 2 & 4 & 6 & 4 & 7 & 449 & 487 & 327 & 259 & 428 & 412 & $\begin{array}{r}170 \\
41\end{array}$ \\
\hline $\begin{array}{l}\text { Limites } \\
\text { Pas de limites }\end{array}$ & 100 & 100 & 100 & 100 & 100 & $\begin{array}{l}85 \\
15\end{array}$ & $\begin{array}{l}74 \\
26\end{array}$ & $\begin{array}{l}79 \\
21\end{array}$ & $\begin{array}{l}87 \\
13\end{array}$ & $\begin{array}{l}85 \\
15\end{array}$ & 100 & 100 & 100 & 100 & 100 & 100 & 100 & 100 & 100 & $\begin{array}{r}1 \\
99\end{array}$ & 100 & $\begin{array}{l}41 \\
59\end{array}$ \\
\hline $\begin{array}{l}\text { Fixe } \\
\text { Variable }\end{array}$ & 100 & 100 & 100 & 100 & 100 & $\begin{array}{l}85 \\
15\end{array}$ & $\begin{array}{l}74 \\
26\end{array}$ & $\begin{array}{r}79 \\
21\end{array}$ & $\begin{array}{l}87 \\
13\end{array}$ & $\begin{array}{l}85 \\
15\end{array}$ & 100 & 100 & 100 & 100 & 100 & 100 & 100 & 100 & 100 & $\begin{array}{r}1 \\
99\end{array}$ & 100 & $\begin{array}{l}48 \\
52\end{array}$ \\
\hline $\begin{array}{l}\text { Avec c. (obligatoire) } \\
\text { Avec c. (volontaire) }\end{array}$ & - & - & & - & & & 20 & . & & & & & & & & & & & & & & \\
\hline $\begin{array}{l}\text { Sans contrainte } \\
\text { Un seul produit }\end{array}$ & $\begin{array}{l}100 \\
100\end{array}$ & $\begin{array}{l}100 \\
100\end{array}$ & $\begin{array}{l}100 \\
100\end{array}$ & $\begin{array}{l}100 \\
100\end{array}$ & $\begin{array}{l}100 \\
100\end{array}$ & $\begin{array}{l}100 \\
100\end{array}$ & $\begin{array}{l}100 \\
100\end{array}$ & $\begin{array}{l}100 \\
100\end{array}$ & $\begin{array}{l}100 \\
100\end{array}$ & $\begin{array}{l}100 \\
100\end{array}$ & $\begin{array}{l}100 \\
100\end{array}$ & $\begin{array}{l}100 \\
100\end{array}$ & $\begin{array}{l}100 \\
100\end{array}$ & $\begin{array}{l}100 \\
100\end{array}$ & $\begin{array}{l}100 \\
100\end{array}$ & $\begin{array}{l}100 \\
100\end{array}$ & $\begin{array}{l}100 \\
100\end{array}$ & $\begin{array}{l}100 \\
100\end{array}$ & $\begin{array}{l}100 \\
100\end{array}$ & $\begin{array}{l}100 \\
100\end{array}$ & $\begin{array}{l}100 \\
100\end{array}$ & $\begin{array}{l}100 \\
100\end{array}$ \\
\hline 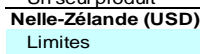 & $\begin{array}{r}4 \\
100\end{array}$ & & & & & & & & & & & & & & & & & & & & & \\
\hline $\begin{array}{l}\text { Pas de limites } \\
\text { Fixe }\end{array}$ & 100 & & & & & & & & & & & & & & & & & & & & & \\
\hline Variable & & & & & & & & & & & & & & & & & & & & & & \\
\hline $\begin{array}{l}\text { Avec c. (obligatoire) } \\
\text { Avec c. (volontaire) } \\
\text { Sans contrainte }\end{array}$ & 100 & & & & & & & & & & & & & & & & & & & & & \\
\hline $\begin{array}{l}\text { Un sueul produtt } \\
\text { Norvège (USD) }\end{array}$ & 570 & 708 & 758 & 715 & 870 & 833 & 783 & 643 & 604 & 599 & 549 & $\begin{array}{lll}477 \\
\end{array}$ & 427 & 402 & 322 & 314 & 376 & 233 & 228 & 239 & 223 & 255 \\
\hline $\begin{array}{l}\text { Limites } \\
\text { Pas de limites }\end{array}$ & $\begin{array}{l}64 \\
36\end{array}$ & $\begin{array}{l}64 \\
36\end{array}$ & $\begin{array}{l}65 \\
35\end{array}$ & $\begin{array}{l}66 \\
34\end{array}$ & $\begin{array}{l}66 \\
34\end{array}$ & $\begin{array}{l}63 \\
37\end{array}$ & $\begin{array}{l}64 \\
36\end{array}$ & $\begin{array}{l}65 \\
35\end{array}$ & $\begin{array}{l}64 \\
36\end{array}$ & $\begin{array}{l}65 \\
35\end{array}$ & $\begin{array}{l}64 \\
36\end{array}$ & $\begin{array}{l}64 \\
36\end{array}$ & $\begin{array}{l}63 \\
37 \\
3\end{array}$ & $\begin{array}{l}63 \\
37\end{array}$ & $\begin{array}{l}65 \\
35 \\
35\end{array}>$ & $\begin{array}{l}59 \\
41\end{array}$ & $\begin{array}{l}60 \\
40\end{array}-10$ & $\begin{array}{l}29 \\
71\end{array}$ & $\begin{array}{l}30 \\
70\end{array}$ & $\begin{array}{l}29 \\
71\end{array}$ & $\begin{array}{r}30 \\
70\end{array}$ & $\begin{array}{l}33 \\
67\end{array}$ \\
\hline $\begin{array}{l}\text { Fixe } \\
\text { Varizble }\end{array}$ & 100 & 100 & 100 & 100 & 100 & 100 & 100 & 100 & 100 & 100 & $\begin{array}{r}30 \\
100-2 l\end{array}$ & $\begin{array}{r}36 \\
100\end{array}$ & 100 & $\begin{array}{r}38 \\
100\end{array}$ & $\begin{array}{r}35 \\
100\end{array}$ & $\begin{array}{r}41 \\
100-200\end{array}$ & $\begin{array}{r}400 \\
100-2 x-5\end{array}$ & 100 & 100 & 100 & 100 & $\begin{array}{r}67 \\
100\end{array}$ \\
\hline $\begin{array}{l}\text { Avec c. (obligatoire) } \\
\text { Avecc (volontaire) }\end{array}$ & & & & & & $-5+2>$ & & & & & & & & & & & - & $-7+2>$ & - & & & \\
\hline $\begin{array}{l}\text { Avec C. CVolontalit } \\
\text { Sans contrainte }\end{array}$ & 100 & 100 & $100^{-}$ & 100 & 100 & 100 & 100 & 100 & 100 & 100 & 100 & 100 & 100 & 100 & 100 & 100 & 100 & 100 & 100 & 100 & 100 & 100 \\
\hline Suisse (USD) & $\frac{100}{23}$ & $\frac{100}{28}$ & $\frac{100}{29}$ & $\frac{100}{26}$ & $\frac{100}{33}$ & $\frac{100}{31}$ & $\frac{100}{40}$ & $\frac{100}{39}$ & $\frac{100}{45}$ & $\frac{100}{54}$ & $\frac{100}{70}$ & $\frac{100}{69}$ & $\frac{100}{80}$ & $\frac{100}{173}$ & $\begin{array}{l}100 \\
196\end{array}$ & $\frac{100}{226}$ & $\frac{100}{233}$ & $\frac{100}{260}$ & $\frac{100}{267}$ & $\frac{100}{265}$ & $\frac{100}{273}$ & $\frac{100}{241}$ \\
\hline $\begin{array}{l}\text { Limites } \\
\text { Pamites }\end{array}$ & 100 & 100 & 100 & 100 & 100 & 100 & 100 & 100 & 100 & 100 & 100 & 100 & 100 & 100 & 100 & 100 & 100 & 100 & 100 & 100 & 100 & 100 \\
\hline $\begin{array}{l}\text { Pas de limites } \\
\text { Fixe } \\
\text { Variable }\end{array}$ & 100 & 100 & 100 & 100 & 100 & 100 & 100 & 100 & 100 & 100 & 100 & 100 & 100 & 100 & 100 & 100 & 100 & 100 & 100 & 100 & 100 & 100 \\
\hline $\begin{array}{l}\text { Avec c. (obligatoire) } \\
\text { Avec c. (volontaire) }\end{array}$ & 100 & 100 & 100 & 100 & 100 & 100 & 100 & 100 & 100 & 100 & 73 & & & & & & & & & & & \\
\hline $\begin{array}{l}\text { Avecc c. CVolontair } \\
\text { Sans contrainte }\end{array}$ & & & & & & & & & & & 27 & $\begin{array}{l}04 \\
36\end{array}$ & 45 & $\begin{array}{l}20 \\
74\end{array}$ & $\begin{array}{l}15 \\
85\end{array}$ & 87 & 88 & 88 & 88 & 87 & 87 & $\begin{array}{r}12 \\
88\end{array}$ \\
\hline $\begin{array}{l}\text { Un seul produit } \\
\text { Turquie (ISD) }\end{array}$ & 100 & 100 & 100 & 100 & 100 & 100 & 100 & 100 & 100 & 100 & 100 & 100 & 100 & 100 & 100 & 100 & 100 & 100 & 100 & 100 & $\begin{array}{r}100 \\
\end{array}$ & $\begin{array}{r}100 \\
1713\end{array}$ \\
\hline $\begin{array}{l}\text { Turquie (USD) } \\
\text { Limites }\end{array}$ & & $\begin{array}{r}19 \\
100\end{array}$ & $\begin{array}{r}13 \\
100\end{array}$ & $\begin{array}{r}19 \\
100\end{array}$ & $\begin{array}{r}46 \\
100\end{array}$ & $\begin{array}{r}32 \\
100\end{array}$ & $\begin{array}{r}21 \\
100\end{array}$ & $\begin{array}{l}435 \\
100\end{array}$ & $\begin{array}{r}34 \\
100\end{array}$ & $\begin{array}{r}61 \\
100\end{array}$ & $\begin{array}{r}80 \\
100\end{array}$ & $\begin{array}{l}134 \\
100\end{array}$ & $\begin{array}{r}45 \\
100\end{array}$ & $\begin{array}{l}268 \\
100\end{array}$ & $\begin{array}{l}357 \\
100\end{array}$ & $\begin{array}{l}454 \\
100\end{array}$ & $\begin{array}{l}135 \\
100\end{array}$ & $\begin{array}{l}205 \\
100\end{array}$ & $\begin{array}{l}346 \\
100\end{array}$ & $\begin{array}{l}804 \\
100\end{array}$ & $\begin{array}{r}1205 \\
100\end{array}$ & 100 \\
\hline Fixe & & 100 & 100 & 100 & 100 & 100 & 100 & 100 & 100 & 100 & 100 & 100 & 100 & 100 & 100 & 100 & 100 & 100 & 100 & 100 & 100 & 100 \\
\hline (obligatoi & & & & & & & & & & & & & & & & & & & & & & \\
\hline $\begin{array}{l}\text { Avecc c. volontair } \\
\text { Sans contrainte }\end{array}$ & & 100 & 100 & 100 & 100 & 100 & 100 & 100 & 100 & 100 & 100 & 100 & 100 & 100 & 100 & 100 & 100 & 100 & 100 & 100 & 100 & 100 \\
\hline 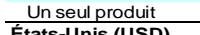 & & & 100 & & & & & & 100 & 100 & 100 & 100 & 100 & 100 & 100 & & & & & 100 & 100 & 100 \\
\hline États-Unis (USD) & 4832 & 2578 & 1338 & 721 & 711 & 192 & 418 & 348 & 308 & 67 & 58 & 327 & 4254 & 10512 & 10291 & 9337 & 1831 & 2872 & 5846 & 6141 & 1904 & $\begin{aligned} 463 \\
36\end{aligned}$ \\
\hline $\begin{array}{l}\text { Limites } \\
\text { Pas de limites }\end{array}$ & & 100 & & 100 & 100 & & & 100 & 100 & 100 & $\begin{array}{r}0 \\
100\end{array}$ & $\begin{array}{l}51 \\
49\end{array}$ & $\begin{array}{l}90 \\
10\end{array}$ & $\begin{array}{l}83 \\
17\end{array}$ & $\begin{array}{l}69 \\
31\end{array}$ & $\begin{array}{l}74 \\
26\end{array}$ & $\begin{array}{l}43 \\
57\end{array}$ & $\begin{array}{l}84 \\
16\end{array}$ & 27 & $\begin{array}{l}79 \\
21\end{array}$ & $\begin{array}{l}31 \\
69\end{array}$ & $\begin{array}{l}36 \\
64\end{array}$ \\
\hline $\begin{array}{l}\text { Fixe } \\
\text { Variable }\end{array}$ & 100 & 100 & 100 & 100 & 100 & 100 & 100 & 100 & 100 & 100 & 100 & 100 & 100 & $\begin{array}{r}0 \\
100\end{array}$ & $\begin{array}{r}1 \\
99\end{array}$ & $\begin{array}{r}0 \\
100\end{array}$ & 100 & $\begin{array}{r}0 \\
100\end{array}$ & $\begin{array}{r}0 \\
100\end{array}$ & $\begin{array}{r}0 \\
100\end{array}$ & 100 & 100 \\
\hline Avec c. (obligatoire) & 100 & 100 & 100 & 100 & 100 & 100 & 100 & 100 & 100 & 100 & 100 & 100 & 100 & 100 & 99 & 100 & 97 & 100 & 100 & 100 & 100 & 100 \\
\hline $\begin{array}{l}\text { Avec c. (vololataire) } \\
\text { Sans contrainte }\end{array}$ & & & & & & & & & & & & & & & & & & & & 0 & & \\
\hline Un seul produit & 100 & 100 & 100 & 100 & 100 & 100 & 100 & 100 & 100 & 100 & 100 & 100 & 100 & 100 & 100 & 100 & 100 & 100 & 100 & 100 & 100 & 100 \\
\hline
\end{tabular}


Tableau annexe 3. Catégorie B1 (PIV) : composition de la catégorie par pays et étiquette, 1986-2007

$$
\text { Part de la catégorie en pourcentage }
$$

\begin{tabular}{|c|c|c|c|c|c|c|c|c|c|c|c|c|c|c|c|c|c|c|c|c|c|c|}
\hline & 1986 & 1987 & 1988 & 1989 & 1990 & 1991 & 1992 & 1993 & $\frac{1994}{1997}$ & $\frac{1995}{11995}$ & 1996 & 1997 & & 1999 & 2000 & 2001 & 2002 & 2003 & 2004 & & 2006 & 2007 \\
\hline $\begin{array}{l}\text { OCDE (USD) } \\
\text { Limites } \\
\text { Pas de }\end{array}$ & $\begin{array}{r}9204 \\
1\end{array}$ & $\begin{aligned} 9783 \\
1\end{aligned}$ & 10249 & $\begin{array}{r}11851 \\
11\end{array}$ & 12930 & 11992 & $\begin{array}{c}11641 \\
11\end{array}$ & $\begin{array}{r}10368 \\
1\end{array}$ & $\begin{array}{r}10927 \\
1\end{array}$ & $\begin{array}{r}11236 \\
4\end{array}$ & $\begin{array}{r}11346 \\
4\end{array}$ & 10467 & $\begin{array}{r}9861 \\
4\end{array}$ & 10692 & $\begin{array}{l}9126 \\
4\end{array}$ & 8789 & 8619 & $\begin{array}{r}9841 \\
9\end{array}$ & $\begin{array}{r}10402 \\
8\end{array}$ & $\begin{array}{l}11094 \\
11094\end{array}$ & $\begin{array}{l}1932 \\
19\end{array}$ & $\frac{12222}{7}$ \\
\hline $\begin{array}{l}\text { Pas de limites } \\
\text { Fixe } \\
\text { yxx }\end{array}$ & $\begin{array}{l}99 \\
78\end{array}$ & $\begin{array}{l}99 \\
80\end{array}$ & $\begin{array}{l}99 \\
79 \\
\end{array}$ & $\begin{array}{l}99 \\
81\end{array}$ & $\begin{array}{l}99 \\
83\end{array}$ & $\begin{array}{l}98 \\
80\end{array}$ & $\begin{array}{l}99 \\
78\end{array}$ & $\begin{array}{l}99 \\
78\end{array}$ & $\begin{array}{l}99 \\
74\end{array}$ & $\begin{array}{l}96 \\
72\end{array}$ & $\begin{array}{l}96 \\
74\end{array}$ & $\begin{array}{l}96 \\
75\end{array}$ & $\begin{array}{l}96 \\
76\end{array}$ & $\begin{array}{l}96 \\
75\end{array}$ & $\begin{array}{l}96 \\
78\end{array}$ & $\begin{array}{l}92 \\
81\end{array}$ & $\begin{array}{l}91 \\
81\end{array}$ & $\begin{array}{l}91 \\
82\end{array}$ & $\begin{array}{l}92 \\
92 \\
83\end{array}$ & $\begin{array}{l}92 \\
83\end{array}$ & $\begin{array}{l}92 \\
82\end{array}$ & $\begin{array}{l}93 \\
83\end{array}$ \\
\hline 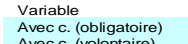 & $\begin{array}{r}22 \\
9\end{array}$ & $\begin{array}{r}20 \\
7\end{array}$ & $\begin{array}{c}21 \\
7\end{array}$ & $\begin{array}{c}19 \\
6\end{array}$ & $\begin{array}{c}17 \\
5\end{array}$ & $\begin{array}{l}20 \\
5\end{array}$ & $\begin{array}{c}22 \\
3\end{array}$ & $\begin{array}{r}22 \\
3\end{array}$ & $\begin{array}{c}26 \\
4\end{array}$ & $\begin{array}{r}28 \\
4\end{array}$ & $\begin{array}{r}26 \\
4\end{array}$ & $\begin{array}{r}25 \\
25 \\
4\end{array}$ & $\begin{array}{r}24 \\
24\end{array}$ & $\begin{array}{l}25 \\
3 \\
3\end{array}$ & $\begin{array}{c}22 \\
22 \\
3\end{array}$ & $\begin{array}{l}0 \\
19 \\
3\end{array}$ & $\begin{array}{c}0 \\
19 \\
3\end{array}$ & $\begin{array}{l}18 \\
18 \\
3\end{array}$ & $\begin{array}{c}17 \\
17 \\
3\end{array}$ & $\begin{array}{c}0 \\
17 \\
2\end{array}$ & $\begin{array}{c}18 \\
2\end{array}$ & $\begin{array}{c}017 \\
17 \\
2\end{array}$ \\
\hline $\begin{array}{l}\text { Avec c. c. volontataire) } \\
\text { Sans contrainte }\end{array}$ & $99^{1}$ & 93 & 93 & & 95 & 95 & $\begin{array}{l}0 \\
97 \\
97\end{array}$ & $\begin{array}{l}00 \\
97\end{array}$ & $\begin{array}{r}0 \\
96\end{array}$ & $\begin{array}{c}0 \\
96\end{array}$ & $\begin{array}{l}00 \\
95\end{array}$ & $\begin{array}{c}0 \\
96\end{array}$ & $\begin{array}{c}0 \\
96\end{array}$ & $\begin{array}{l}0 \\
97\end{array}$ & $\begin{array}{l}1 \\
96\end{array}$ & $\begin{array}{c}0 \\
96\end{array}$ & $\begin{array}{c}0 \\
96\end{array}$ & $\begin{array}{r}0 \\
97\end{array}$ & $\begin{array}{c}1 \\
96\end{array}$ & $\begin{array}{r}2 \\
95 \\
95\end{array}$ & $\begin{array}{r}3 \\
95 \\
95\end{array}$ & $\begin{array}{c}1 \\
97 \\
97\end{array}$ \\
\hline $\begin{array}{l}\text { Un suev loroduit } \\
\text { Un groupe de produits }\end{array}$ & $\begin{array}{l}0 \\
38\end{array}$ & $\begin{array}{r}0 \\
41\end{array}$ & $\begin{array}{l}0 \\
41\end{array}$ & $\begin{array}{r}0 \\
33\end{array}$ & $\begin{array}{l}1 \\
30\end{array}$ & $\begin{array}{l}1 \\
25\end{array}$ & $\begin{array}{l}1 \\
23\end{array}$ & $\begin{array}{l}1 \\
26\end{array}$ & $\begin{array}{l}1 \\
24 \\
\end{array}$ & $\begin{array}{l}1 \\
22 \\
\end{array}$ & $\begin{array}{l}2 \\
24\end{array}$ & $\begin{array}{r}1 \\
22\end{array}$ & $\begin{array}{l}1 \\
21\end{array}$ & $\begin{array}{l}1 \\
20\end{array}$ & $\begin{array}{l}4 \\
18\end{array}$ & $21^{2}$ & $\begin{array}{l}1 \\
17\end{array}$ & $\begin{array}{r}0 \\
19\end{array}$ & $\begin{array}{l}0 \\
15\end{array}$ & $\begin{array}{r}1 \\
15\end{array}$ & $\begin{array}{c}1 \\
15\end{array}$ & $\begin{array}{r}3 \\
13 \\
\end{array}$ \\
\hline $\begin{array}{l}\text { Tous lese rodouctits } \\
\text { Austraie (USD) }\end{array}$ & $\frac{62}{246}$ & $\begin{array}{l}529 \\
222\end{array}$ & $\begin{array}{r}58 \\
183 \\
\end{array}$ & $\frac{67}{219}$ & $\frac{69}{266}$ & $\frac{74}{358}$ & $\begin{array}{r}76 \\
419\end{array}$ & $\frac{73}{351}$ & $\frac{75}{366}$ & $\frac{78}{370}$ & $\frac{74}{465}$ & $\frac{77}{395}$ & $\frac{78}{333}$ & $\frac{80}{393}$ & $\frac{79}{326}$ & $\frac{77}{289}$ & $\frac{82}{315}$ & $\frac{80}{374}$ & $\begin{array}{r}84 \\
429\end{array}$ & $\frac{85}{373}$ & $\frac{84}{572}$ & $\begin{aligned} 85 \\
474\end{aligned}$ \\
\hline $\begin{array}{l}\text { Limites } \\
\text { Pas de limites }\end{array}$ & $100^{\circ}$ & $100^{\circ}-(x)$ & $100^{\circ}$ & $100^{\circ}$ & $100^{\circ}-(x)$ & $100^{\circ}$ & $100^{\circ}$ & $100^{\circ}-(x)$ & $100^{\circ}-(x)$ & $100^{\circ}-(x)$ & $100^{\circ}-(x)$ & $100^{\circ}$ & $100^{\circ}$ & $100^{\circ}$ & $100^{\circ}$ & $100^{\circ}$ & $100^{\circ}$ & $100^{\circ}$ & $100^{\circ}$ & $\begin{array}{c}0 \\
100\end{array}$ & $100^{\circ}$ & $100^{\circ}-(x)$ \\
\hline $\begin{array}{l}\text { Fixe } \\
\text { Variable }\end{array}$ & $100^{\circ}$ & $100^{\circ}-(x)$ & $100^{\circ}$ & $100^{\circ}$ & $100^{\circ}$ & $100^{\circ}$ & $100^{\circ}$ & $100^{\circ}$ & $100^{\circ}-(x)$ & $100^{\circ}-(x)$ & $100^{\circ}$ & $100^{\circ}-(x)$ & $100^{\circ}$ & $100^{\circ}$ & $\begin{array}{l}2 \\
98\end{array}$ & $\begin{array}{l}2 \\
98 \\
98\end{array}$ & $\begin{array}{r}0 \\
100\end{array}$ & $\begin{array}{r}0 \\
100\end{array}$ & $100^{\circ}$ & $100^{\circ}$ & $100^{\circ}$ & $100^{\circ}$ \\
\hline 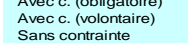 & 100 & 100 & 100 & 100 & 100 & 100 & 100 & 100 & 100 & 100 & 100 & 100 & 100 & 100 & 98 & 98 & 100 & 100 & 100 & 100 & 100 & 100 \\
\hline 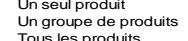 & & & & & & & & & & & & & & & & & & & & & & \\
\hline $\begin{array}{l}\text { Tous less rodouits } \\
\text { Canada (USD) }\end{array}$ & $\begin{array}{l}100 \\
370\end{array}$ & $\frac{100}{472}$ & $\frac{100}{1011}$ & $\begin{array}{l}99 \\
554\end{array}$ & $\begin{array}{l}86 \\
433\end{array}$ & $\begin{array}{l}80 \\
386 \\
386\end{array}$ & $\begin{array}{l}86 \\
346\end{array}$ & $\begin{array}{l}87 \\
307\end{array}$ & $\frac{94}{283}$ & $\frac{91}{253}$ & $\frac{93}{251}$ & $\begin{array}{l}944 \\
249\end{array}$ & $\frac{95}{241}$ & $\frac{100}{244}$ & $\begin{array}{r}98 \\
246\end{array}$ & $\frac{98}{227}$ & $\frac{100}{204}$ & $\frac{100}{247}$ & $\begin{array}{l}\frac{100}{258} \\
258\end{array}$ & $\begin{array}{l}\frac{100}{328} \\
328\end{array}$ & $\frac{100}{378}$ & $\begin{array}{l}\frac{100}{386} \\
386\end{array}$ \\
\hline $\begin{array}{l}\text { Limitises } \\
\text { Pas de limites } \\
\text { Fire lie }\end{array}$ & $\begin{array}{r}5 \\
95 \\
100\end{array}$ & $\begin{array}{r}5 \\
95 \\
100\end{array}$ & $\begin{array}{r}2 \\
98 \\
100\end{array}$ & $\begin{array}{r}0 \\
100 \\
100\end{array}$ & $\begin{array}{r}14 \\
86 \\
100\end{array}$ & $\begin{array}{r}13 \\
87 \\
100\end{array}$ & $\begin{array}{r}11 \\
89 \\
100\end{array}$ & $\begin{array}{r}3 \\
97 \\
970\end{array}$ & $\begin{array}{r}5 \\
95 \\
100\end{array}$ & $\begin{array}{r}5 \\
95 \\
100\end{array}$ & $\begin{array}{r}5 \\
95 \\
9100\end{array}$ & $\begin{array}{r}5 \\
95 \\
100\end{array}$ & $\begin{array}{r}6 \\
94 \\
100\end{array}$ & $\begin{array}{r}6 \\
94 \\
100\end{array}$ & $\begin{array}{r}10 \\
90 \\
100\end{array}$ & $\begin{array}{r}7 \\
93 \\
100\end{array}$ & $\begin{array}{r}6 \\
94 \\
100\end{array}$ & $\begin{array}{r}7 \\
93 \\
100\end{array}$ & $\begin{array}{r}6 \\
94 \\
100\end{array}$ & $\begin{array}{r}7 \\
93 \\
100\end{array}$ & $\begin{array}{r}9 \\
91 \\
100\end{array}$ & $\begin{array}{r}8 \\
92 \\
100\end{array}$ \\
\hline $\begin{array}{l}\text { Variable } \\
\text { Avec c. (obligatoire) }\end{array}$ & & & & & & & & & & & & & & & & & & & & & & \\
\hline 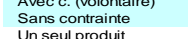 & 100 & 100 & 100 & 100 & 100 & 100 & 100 & 100 & 100 & 100 & 100 & 100 & 100 & 100 & 100 & 100 & 100 & $\begin{array}{l}9 \\
99 \\
\end{array}$ & $\begin{array}{l}1 \\
99 \\
\end{array}$ & 99 & $\begin{array}{l}2 \\
98 \\
\end{array}$ & 98 \\
\hline 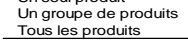 & $\begin{array}{r}27 \\
73 \\
\end{array}$ & $\begin{array}{l}23 \\
77 \\
\end{array}$ & $\begin{array}{l}62 \\
38 \\
\end{array}$ & $\begin{array}{l}13 \\
87 \\
\end{array}$ & $\begin{array}{l}28 \\
72 \\
\end{array}$ & $\begin{array}{l}31 \\
69 \\
\end{array}$ & $\begin{array}{l}30 \\
70 \\
\end{array}$ & $\begin{array}{l}25 \\
75 \\
\end{array}$ & $\begin{array}{l}22 \\
78 \\
\end{array}$ & $\begin{array}{l}15 \\
85 \\
\end{array}$ & $\begin{array}{l}10 \\
90 \\
\end{array}$ & $\begin{array}{l}10 \\
90 \\
\end{array}$ & $\begin{array}{l}12 \\
88 \\
\end{array}$ & $\begin{array}{l}12 \\
88 \\
\end{array}$ & $\begin{array}{l}15 \\
85 \\
\end{array}$ & $\begin{array}{l}12 \\
88\end{array}$ & $\begin{array}{l}11 \\
89\end{array}$ & $\begin{array}{l}12 \\
88 \\
\end{array}$ & $\begin{array}{l}11 \\
89\end{array}$ & $\begin{array}{l}11 \\
89\end{array}$ & $\begin{array}{l}11 \\
89\end{array}$ & $\begin{array}{l}10 \\
90\end{array}$ \\
\hline $\begin{array}{l}\text { UEustes produls } \\
\text { UE (USD) }\end{array}$ & 900 & 1003 & 978 & 2358 & 3591 & 3958 & 3712 & 2427 & 2388 & 2820 & 2967 & 2743 & 2788 & 3483 & 3171 & 2923 & 3136 & 3882 & 4924 & 5347 & 5554 & 5977 \\
\hline $\begin{array}{l}\text { Limites } \\
\text { Pas de limites } \\
\text { Fixe }\end{array}$ & $\begin{array}{l}100 \\
100\end{array}$ & $\begin{array}{l}100 \\
100\end{array}$ & $\begin{array}{l}100 \\
100\end{array}$ & $\begin{array}{l}100 \\
100\end{array}$ & $\begin{array}{l}100^{\circ} \\
100\end{array}$ & $\begin{array}{l}100^{\circ} \\
100\end{array}$ & $\begin{array}{l}100 \\
100\end{array}$ & $\begin{array}{l}100 \\
100\end{array}$ & $\begin{array}{l}100^{\circ} \\
99\end{array}$ & $\begin{aligned} 14 \\
86 \\
100\end{aligned}$ & $\begin{array}{l}13 \\
87 \\
97\end{array}$ & $\begin{array}{l}13 \\
87 \\
98\end{array}$ & $\begin{array}{l}12 \\
88 \\
97 \\
97\end{array}$ & $\begin{array}{l}10 \\
90 \\
99\end{array}$ & $\begin{array}{l}99 \\
91 \\
91\end{array}$ & $\begin{array}{l}10 \\
90 \\
95\end{array}$ & $\begin{array}{l}10 \\
90 \\
98\end{array}$ & $\begin{array}{r}99 \\
91 \\
100\end{array}$ & $\begin{array}{c}96 \\
94 \\
100\end{array}$ & $\begin{array}{r}96 \\
94 \\
100\end{array}$ & $\begin{array}{r}93 \\
97 \\
100\end{array}$ & $99^{3}$ \\
\hline $\begin{array}{l}\text { variable } \\
\text { Avero. (obigatoire) }\end{array}$ & & & & & & & & & & & ${ }_{37}^{97}$ & $\begin{array}{r}98 \\
2\end{array}$ & ${ }_{3}^{97}$ & & $\begin{array}{r}91 \\
9\end{array}$ & $\begin{array}{r}95 \\
5\end{array}$ & $\begin{array}{r}98 \\
2\end{array}$ & $\begin{array}{r}100 \\
(0)\end{array}$ & & $\begin{aligned} 100 \\
0\end{aligned}$ & $\begin{array}{r}100 \\
0\end{array}$ & $\begin{array}{c}100 \\
\text { (0) }\end{array}$ \\
\hline $\begin{array}{l}\text { Avec c. (vilonotiare)' } \\
\text { Sans contrainte }\end{array}$ & 100 & $100^{\circ}$ & $100^{\circ}$ & $100^{\circ}$ & $100^{\circ}$ & $100^{\circ}$ & $100^{\circ}$ & 100 & 100 & $\begin{array}{r}0 \\
100\end{array}$ & $\begin{array}{l}1 \\
99 \\
\end{array}$ & $100^{\circ}$ & $100^{\circ}$ & $\begin{array}{l}1 \\
99 \\
\end{array}$ & $\begin{array}{l}1 \\
99 \\
\end{array}$ & $\begin{array}{l}1 \\
99 \\
\end{array}$ & $\begin{array}{l}1 \\
99 \\
\end{array}$ & 99 & 99 & 99 & $\begin{array}{l}2 \\
98 \\
\end{array}$ & $\begin{array}{l}1 \\
99 \\
\end{array}$ \\
\hline 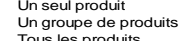 & 51 & ${ }_{5}^{49}$ & $\begin{array}{l}48 \\
52\end{array}$ & $\begin{array}{l}21 \\
79\end{array}$ & $\begin{array}{l}21 \\
790\end{array}$ & 19 & 19 & $\begin{array}{l}18 \\
88\end{array}$ & $\begin{array}{l}15 \\
15 \\
14\end{array}$ & $\begin{array}{l}0 \\
23 \\
736\end{array}$ & $\begin{array}{l}3 \\
21\end{array}$ & $1_{19}^{2}$ & $\begin{array}{r}3 \\
20\end{array}$ & 16 & $\begin{array}{r}99 \\
15\end{array}$ & $\begin{array}{r}5 \\
17 \\
17\end{array}$ & $\frac{2}{16}$ & $\begin{array}{l}(0) \\
17\end{array}$ & $\begin{array}{l}0 \\
14\end{array}$ & $\begin{array}{r}0 \\
12\end{array}$ & $\begin{array}{r}0 \\
10 \\
10\end{array}$ & $\begin{array}{c}(0) \\
11\end{array}$ \\
\hline $\begin{array}{l}\text { Tous les produitits } \\
\text { siande (USD) }\end{array}$ & $\begin{array}{r}49 \\
5\end{array}$ & $\frac{51}{3}$ & $\frac{52}{2}$ & $\frac{79}{1}$ & $\frac{79}{4}$ & $\frac{81}{1}$ & $\frac{81}{0}$ & $\frac{82}{0}$ & $\frac{84}{0}$ & $\begin{array}{c}\frac{76}{0} \\
\end{array}$ & $\begin{array}{c}76 \\
0\end{array}$ & $\frac{78}{0}$ & $\frac{78}{0}$ & & $\frac{76}{0}$ & $\frac{78}{0}$ & & $\frac{83}{1}$ & & $\frac{88}{2}$ & $\frac{90}{2}$ & $\frac{89}{2}$ \\
\hline $\begin{array}{l}\text { Limites } \\
\text { Pas de limites }\end{array}$ & 100 & $100^{\circ}$ & 100 & 100 & 100 & $100^{\circ}-(x)$ & 100 & 100 & 100 & 100 & 100 & 100 & 100 & 100 & 100 & 100 & 100 & 100 & 100 & 100 & 100 & 100 \\
\hline & $\begin{array}{l}11 \\
89\end{array}$ & $\begin{array}{l}3 \\
97\end{array}$ & $\begin{array}{r}5 \\
95\end{array}$ & $\begin{array}{l}44 \\
56\end{array}$ & $\begin{array}{r}5 \\
95 \\
\end{array}$ & 100 & 100 & 100 & 100 & 100 & 100 & 100 & 100 & 100 & 100 & 100 & 100 & 100 & 100 & 100 & 100 & 100 \\
\hline $\begin{array}{l}\text { Avec c. (obligatiore) } \\
\text { Avec c (volontalere) }\end{array}$ & & & & & & & & & & & & & & & & & & & & & & \\
\hline $\begin{array}{l}\text { Sans contraintel } \\
\text { Un seut proudit }\end{array}$ & 100 & 100 & 100 & 100 & 100 & 100 & 100 & 100 & 100 & 100 & 100 & 100 & 100 & 100 & 100 & 100 & 100 & 100 & 100 & 100 & 100 & 100 \\
\hline 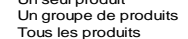 & & & & & $\begin{array}{l}81 \\
119\end{array}$ & & & & & & & & & & & & & & & & & \\
\hline 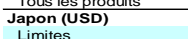 & 918 & $\frac{100}{1047}$ & 1106 & 963 & $\frac{19}{910}$ & $\frac{100}{1000}$ & $\frac{100}{1080}$ & $\frac{100}{1186}$ & $\frac{1200}{1240}$ & $\frac{100}{1329}$ & $\frac{100}{1114}$ & $\frac{1000}{1047}$ & 753 & $\begin{array}{l}100 \\
830 \\
30\end{array}$ & 797 & $\frac{100}{664}$ & $\frac{100}{633}$ & $\frac{100}{610}$ & $\frac{100}{636}$ & $\frac{100}{630}$ & $\frac{100}{607}$ & $\frac{100}{619}$ \\
\hline $\begin{array}{l}\text { Pas de limites } \\
\text { Fis de }\end{array}$ & 100 & 100 & 100 & 100 & 100 & 100 & 100 & 100 & 100 & $100^{\circ}$ & 100 & 100 & $100^{\circ}$ & 100 & 100 & 100 & 100 & 100 & 100 & $100^{\circ}-(x)$ & 100 & $100^{\circ}$ \\
\hline $\begin{array}{l}\text { Fixe } \\
\text { Variable }\end{array}$ & $\begin{array}{l}39 \\
61\end{array}$ & $\begin{array}{l}39 \\
61\end{array}$ & $\begin{array}{l}40 \\
60\end{array}$ & $\begin{array}{l}38 \\
62\end{array}$ & $\begin{array}{l}37 \\
63\end{array}$ & $\begin{array}{l}39 \\
61\end{array}$ & $\begin{array}{l}38 \\
62 \\
62\end{array}$ & $\begin{array}{l}34 \\
66\end{array}$ & $\begin{array}{l}35 \\
65\end{array}$ & $\begin{array}{l}36 \\
64\end{array}$ & $\begin{array}{l}34 \\
66\end{array}$ & $\begin{array}{l}36 \\
64\end{array}$ & $\begin{array}{l}21 \\
79\end{array}$ & $\begin{array}{l}18 \\
82\end{array}$ & $\begin{array}{l}15 \\
85\end{array}$ & $\begin{array}{l}12 \\
88\end{array}$ & 99 & 93 & $\begin{array}{l}6 \\
94\end{array}$ & $\begin{array}{r}5 \\
95\end{array}$ & $\begin{array}{l}6 \\
94\end{array}$ & $\begin{array}{l}12 \\
88\end{array}$ \\
\hline $\begin{array}{l}\text { Avec c. (volontaire) } \\
\text { Sans contrainte }\end{array}$ & 100 & 100 & 100 & 100 & 100 & 100 & 100 & 100 & 100 & 100 & 100 & 100 & 100 & 100 & 100 & 100 & 100 & 100 & 100 & 100 & 100 & 100 \\
\hline 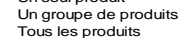 & 100 & 100 & 100 & 100 & 100 & 100 & 100 & 100 & 100 & 100 & 100 & 100 & 100 & 100 & 100 & 100 & 100 & 100 & & & 100 & 100 \\
\hline Coré (USL & 13 & 36 & 29 & 87 & 79 & 72 & 103 & 126 & 139 & 110 & 148 & 186 & 147 & 186 & 149 & 161 & 276 & 250 & 234 & 301 & 301 & 406 \\
\hline $\begin{array}{l}\text { limites limites } \\
\text { Pas de limites } \\
\text { Fixe }\end{array}$ & $\begin{array}{l}100 \\
100\end{array}$ & $\begin{array}{l}100 \\
100\end{array}$ & $\begin{array}{l}100 \\
100\end{array}$ & $\begin{array}{l}100 \\
100\end{array}$ & $\begin{array}{l}100 \\
100\end{array}$ & $\begin{array}{l}100 \\
100\end{array}$ & $\begin{array}{l}100 \\
100\end{array}$ & $\begin{array}{l}100 \\
100\end{array}$ & $\begin{array}{l}100 \\
100\end{array}$ & $\begin{array}{l}100 \\
100\end{array}$ & $\begin{array}{l}100 \\
100\end{array}$ & $\begin{array}{l}100 \\
100\end{array}$ & $\begin{array}{l}100 \\
100\end{array}$ & $\begin{array}{l}100 \\
100\end{array}$ & $\begin{array}{l}100 \\
100\end{array}$ & $\begin{array}{l}100 \\
100\end{array}$ & $\begin{array}{l}100 \\
100\end{array}$ & $\begin{array}{l}100^{\circ} \\
100\end{array}$ & $\begin{array}{l}100 \\
100\end{array}$ & $\begin{array}{l}100 \\
100\end{array}$ & $\begin{array}{l}100 \\
100\end{array}$ & $\begin{array}{l}100^{\circ} \\
100\end{array}$ \\
\hline 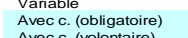 & & & & & & & & & & & & & & & & & & & & & & \\
\hline Sa & $100^{\circ}$ & 100 & 100 & $100^{\circ}$ & $100^{\circ}$ & 100 & $100^{\circ}$ & 100 & 100 & 100 & 100 & 100 & 100 & 100 & 100 & 100 & 100 & $100^{\circ}$ & $100^{\circ}$ & $100^{\circ}$ & $100^{\circ}$ & $100^{\circ}$ \\
\hline produits & 100 & $100^{\circ}$ & 100 & 100 & 100 & 100 & 100 & 100 & $100^{\circ}$ & 100 & 100 & $100^{\circ}$ & 100 & 100 & 100 & 100 & $100^{\circ}$ & $100^{\circ}$ & 100 & 100 & 100 & $100^{\circ}$ \\
\hline
\end{tabular}

ANALYSE DE LA COMPOSITION DU SOUTIEN AUX PRODUCTEURS : NOUVEAUX OUTILS ET MÉTHODES @ OECD 2010 
Tableau annexe 3. Catégorie B1 (PIV) : composition de la catégorie par pays et étiquette, 1986-2007 (suite)

\begin{tabular}{|c|c|c|c|c|c|c|c|c|c|c|c|c|c|c|c|c|c|c|c|c|c|c|}
\hline Mexique (USD) & $\begin{array}{c}1986 \\
34\end{array}$ & $\begin{array}{r}1987 \\
950\end{array}$ & $\begin{array}{r}1988 \\
888\end{array}$ & $\begin{array}{l}\frac{1989}{1167} \\
\end{array}$ & $\begin{array}{l}1990 \\
2011\end{array}$ & $\begin{aligned} 1991 \\
830\end{aligned}$ & $\frac{1992}{588}$ & \begin{tabular}{|l}
1993 \\
222
\end{tabular} & $\begin{array}{r}1994 \\
991\end{array}$ & $\begin{array}{r}1995 \\
438\end{array}$ & $\begin{array}{r}1996 \\
490\end{array}$ & 1997 & 1998 & 1999 & $\frac{2000}{101}$ & $\begin{array}{r}2001 \\
479\end{array}$ & $\frac{2002}{468}$ & $\frac{2003}{609}$ & $\frac{2004}{578}$ & $\frac{2005}{710}$ & 2006 & $\frac{2007}{1028}$ \\
\hline $\begin{array}{l}\text { Limitis } \\
\text { Pas de limites }\end{array}$ & 100 & 100 & 100 & 100 & 100 & 100 & 100 & 100 & 100 & 100 & 100 & $100^{\circ}-(-3)$ & 100 & $100^{\circ}-(-3)$ & $100^{\circ}-(-2)$ & $\begin{array}{l}72 \\
28\end{array}$ & $\begin{array}{l}83 \\
17\end{array}$ & $\begin{array}{l}83 \\
17\end{array}$ & $\begin{array}{l}84 \\
16\end{array}$ & $\begin{array}{l}80 \\
20\end{array}$ & $\begin{array}{l}77 \\
23\end{array}$ & $\begin{array}{l}56 \\
44\end{array}$ \\
\hline $\begin{array}{l}\text { Fie } \\
\text { Variable }\end{array}$ & 100 & 100 & 100 & 100 & 100 & 100 & 100 & 100 & 100 & 100 & 100 & 100 & 100 & 100 & 100 & $\begin{array}{l}28 \\
72\end{array}$ & $\begin{array}{l}17 \\
83\end{array}$ & $\begin{array}{l}17 \\
83\end{array}$ & $\begin{array}{l}16 \\
84\end{array}$ & $\begin{array}{l}20 \\
80\end{array}$ & $\begin{array}{l}23 \\
77\end{array}$ & $\begin{array}{l}44 \\
56\end{array}$ \\
\hline $\begin{array}{l}\text { Avec c. (obligatoire) } \\
\text { Avec c. (volontaire) }\end{array}$ & & & & & & & & & & & & & & & & & & & & & & \\
\hline $\begin{array}{l}\text { Uns seul produit } \\
\text { Un sut }\end{array}$ & 100 & 100 & 100 & 100 & 100 & 100 & 100 & $\begin{array}{r}100 \\
2\end{array}$ & $\begin{array}{r}100 \\
4\end{array}$ & $\begin{array}{r}100 \\
5\end{array}$ & $\begin{array}{r}100 \\
1\end{array}$ & $\begin{array}{r}100 \\
0\end{array}$ & $\begin{array}{r}100 \\
0\end{array}$ & 100 & 100 & 100 & 100 & 100 & 100 & $\begin{array}{r}100 \\
5\end{array}$ & $\begin{array}{r}100 \\
9\end{array}$ & $\begin{array}{r}100 \\
28\end{array}$ \\
\hline $\begin{array}{l}\text { Ongroupe de prodouts } \\
\text { Tous les produits }\end{array}$ & $\begin{array}{l}82 \\
18 \\
5\end{array}$ & 8 & $\begin{array}{l}87 \\
13 \\
\end{array}$ & 43 & $\begin{array}{l}19 \\
29 \\
\end{array}$ & 0 & $\begin{array}{r}96 \\
4\end{array}$ & $\begin{array}{r}95 \\
3\end{array}$ & 90 & $\begin{array}{r}88 \\
7\end{array}$ & $\begin{array}{r}93 \\
6 \\
\end{array}$ & $\begin{array}{r}54 \\
46 \\
\end{array}$ & $\begin{array}{l}39 \\
60 \\
6\end{array}$ & $\begin{array}{l}56 \\
44 \\
\end{array}$ & $\begin{array}{l}46 \\
54\end{array}$ & $\begin{array}{l}18 \\
82\end{array}$ & 93 & $\begin{array}{l}12 \\
88 \\
\end{array}$ & 91 & 87 & 82 & $\begin{array}{l}11 \\
61 \\
\end{array}$ \\
\hline $\begin{array}{l}\text { Limites } \\
\text { Pas de limites }\end{array}$ & 100 & & & & & & & & & & & & & & & & & & & & & \\
\hline $\begin{array}{l}\text { Fixe } \\
\text { Variable }\end{array}$ & 100 & & & & & & & & & & & & & & & & & & & & & \\
\hline $\begin{array}{l}\text { Avec c. (obligatoire) } \\
\text { Avec c. (volontaire) }\end{array}$ & & & & & & & & & & & & & & & & & & & & & & \\
\hline $\begin{array}{l}\text { Sans contrainte } \\
\text { Un seul produit } \\
\text { Un groupe de produits }\end{array}$ & 100 & & & & & & & & & & & & & & & & & & & & & \\
\hline $\begin{array}{l}\text { Tous les produits } \\
\text { Norvège (USD) }\end{array}$ & $\frac{17}{140}$ & 147 & 158 & 153 & 98 & 83 & 74 & 63 & 61 & 74 & 88 & 87 & 89 & 87 & 74 & 71 & 68 & 78 & 85 & 93 & 89 & 101 \\
\hline $\begin{array}{l}\text { Limites } \\
\text { Pas de limites }\end{array}$ & $100^{\circ}$ & $100^{\circ}-(-3)$ & $100^{\circ}$ & $100^{\circ}-(-3)$ & $100^{\circ}-(-3)$ & $100^{\circ}-(-2)$ & 100 & $100^{\circ}$ & $100^{\circ}$ & $100^{\circ}$ & $100^{\circ}$ & $100^{\circ}-(-2)$ & $100^{\circ}-(-3)$ & 100 & $100^{\circ}$ & 100 & $100^{\circ}$ & $100^{\circ}-(-3)$ & 100 & $100^{\circ}-(x-3)$ & 100 & $100^{\circ}$ \\
\hline $\begin{array}{l}\text { Variable } \\
\text { varial }\end{array}$ & $\begin{array}{r}8 \\
92\end{array}$ & 92 & 92 & $\begin{array}{r}8 \\
92 \\
\end{array}$ & $\begin{array}{l}12 \\
88\end{array}$ & $\begin{array}{l}13 \\
87\end{array}$ & $\begin{array}{l}15 \\
85\end{array}$ & $\begin{array}{l}17 \\
83\end{array}$ & $\begin{array}{l}16 \\
84\end{array}$ & $\begin{array}{l}30 \\
70\end{array}$ & $\begin{array}{l}41 \\
59\end{array}$ & $\begin{array}{l}40 \\
60\end{array}$ & $\begin{array}{l}40 \\
60\end{array}$ & $\begin{array}{l}41 \\
59\end{array}$ & $\begin{array}{l}37 \\
63\end{array}$ & $\begin{array}{l}38 \\
62\end{array}$ & $\begin{array}{l}38 \\
62\end{array}$ & $\begin{array}{l}35 \\
65 \\
6\end{array}$ & $\begin{array}{l}35 \\
65\end{array}$ & $\begin{array}{l}33 \\
67\end{array}$ & $\begin{array}{l}29 \\
71\end{array}$ & $\begin{array}{l}32 \\
68\end{array}$ \\
\hline $\begin{array}{l}\text { Avec c. (volontaire) } \\
\text { Sans contrainte }\end{array}$ & 100 & 100 & 100 & 100 & 100 & 100 & 99 & $\begin{array}{l}2 \\
98\end{array}$ & 98 & 99 & 100 & 100 & 100 & 100 & 100 & 100 & 100 & 100 & 100 & 100 & 100 & 100 \\
\hline $\begin{array}{l}\text { Un seul produit } \\
\text { Un groupe de produits }\end{array}$ & 76 & 73 & 72 & 71 & 52 & 44 & 33 & 31 & 30 & 30 & 26 & 26 & 24 & 24 & 22 & 23 & 29 & 27 & 27 & 27 & 24 & 27 \\
\hline $\begin{array}{l}\text { Tous les produits } \\
\text { Suisse (USD) }\end{array}$ & 258 & 328 & 280 & 179 & $\frac{48}{122}$ & 125 & 127 & 220 & 273 & 279 & 239 & 208 & 192 & 74 & 68 & 64 & 70 & 82 & 88 & 84 & 85 & 96 \\
\hline $\begin{array}{l}\text { Pas de limites } \\
\text { Fixe }\end{array}$ & $\begin{array}{l}100 \\
100\end{array}$ & $\begin{array}{l}100 \\
100\end{array}$ & $\begin{array}{l}100 \\
100\end{array}$ & $\begin{array}{l}100 \\
100\end{array}$ & $\begin{array}{l}100 \\
100\end{array}$ & $\begin{array}{l}100 \\
100\end{array}$ & $\begin{array}{l}100 \\
100\end{array}$ & $\begin{array}{l}100 \\
100\end{array}$ & $\begin{array}{l}100 \\
100\end{array}$ & $\begin{array}{l}100 \\
100\end{array}$ & $\begin{array}{l}100 \\
100\end{array}$ & $\begin{array}{l}100 \\
100\end{array}$ & $\begin{array}{l}100 \\
100\end{array}$ & $\begin{array}{l}100 \\
100\end{array}$ & $\begin{array}{l}100 \\
100\end{array}$ & $\begin{array}{l}100 \\
100\end{array}$ & $\begin{array}{l}100 \\
100\end{array}$ & $\begin{array}{l}100 \\
100\end{array}$ & $\begin{array}{l}100 \\
100\end{array}$ & $\begin{array}{l}100 \\
100\end{array}$ & $\begin{array}{l}100 \\
100\end{array}$ & $\begin{array}{l}100 \\
100\end{array}$ \\
\hline $\begin{array}{l}\text { Variable } \\
\text { Avec C. (obligatoire) }\end{array}$ & & & & & & & & & & 48 & & & 60 & & & & & & & & & \\
\hline $\begin{array}{l}\text { Avec c. (. Volontaire) } \\
\text { Sans contrainte }\end{array}$ & 100 & 100 & 100 & 100 & 100 & 100 & 100 & 62 & $\begin{array}{l}3 \\
50\end{array}$ & $\begin{array}{r}4 \\
47\end{array}$ & $\begin{array}{r}66 \\
39\end{array}$ & $\begin{array}{r}6 \\
39 \\
\end{array}$ & $\begin{array}{r}7 \\
33\end{array}$ & $\begin{array}{l}21 \\
79\end{array}$ & $\begin{array}{l}19 \\
81\end{array}$ & $\begin{array}{l}20 \\
80\end{array}$ & $\begin{array}{l}21 \\
79\end{array}$ & $\begin{array}{l}20 \\
80\end{array}$ & $\begin{array}{l}20 \\
80\end{array}$ & $\begin{array}{l}20 \\
80\end{array}$ & $\begin{array}{l}20 \\
80\end{array}$ & $\begin{array}{l}19 \\
81\end{array}$ \\
\hline $\begin{array}{l}\text { Un seul produit } \\
\text { Un ropune de }\end{array}$ & $\begin{array}{r}7 \\
83\end{array}$ & $\begin{array}{r}7 \\
84\end{array}$ & $\begin{array}{r}8 \\
80\end{array}$ & 12 & $\begin{array}{l}21 \\
52\end{array}$ & $\begin{array}{l}25 \\
38\end{array}$ & $\begin{array}{l}30 \\
33\end{array}$ & ${ }_{66}^{13}$ & $\begin{array}{l}13 \\
69\end{array}$ & $\begin{array}{r}5 \\
74\end{array}$ & 11 & $\begin{array}{l}12 \\
65 \\
65\end{array}$ & 75 & $\begin{array}{l}10 \\
30\end{array}$ & 17 & 16 & 17 & 18 & 18 & 16 & 16 & 23 \\
\hline Tous les produits & 10 & $\begin{array}{r}04 \\
9\end{array}$ & 11 & 16 & 27 & 37 & 37 & 21 & 18 & 21 & 24 & 23 & 24 & 60 & 59 & 62 & 61 & 61 & 61 & 63 & 63 & $\begin{array}{r}20 \\
58 \\
\end{array}$ \\
\hline $\begin{array}{l}\text { Turquie (USD) } \\
\end{array}$ & 703 & 879 & 968 & 1165 & 1128 & 1149 & 1458 & 1257 & 1663 & 1952 & 2004 & 1931 & 1756 & 1639 & 723 & 120 & 63 & 66 & 71 & 58 & 135 & 205 \\
\hline Pas de limites & 100 & 100 & 100 & 100 & 100 & 100 & 100 & 100 & 100 & 100 & 100 & 100 & 100 & 100 & 100 & 100 & 100 & 100 & 100 & 100 & 100 & 100 \\
\hline $\begin{array}{l}\text { Fixe } \\
\text { Variable }\end{array}$ & $\begin{array}{l}65 \\
35\end{array}$ & $\begin{array}{l}64 \\
36\end{array}$ & $\begin{array}{l}52 \\
48\end{array}$ & $\begin{array}{r}52 \\
48\end{array}$ & $\begin{array}{l}42 \\
58\end{array}$ & $\begin{array}{l}34 \\
66\end{array}$ & $\begin{array}{l}28 \\
72\end{array}$ & $\begin{array}{l}31 \\
69\end{array}$ & $\begin{array}{l}19 \\
81\end{array}$ & $\begin{array}{l}18 \\
82\end{array}$ & $\begin{array}{l}32 \\
68\end{array}$ & $\begin{array}{l}38 \\
62\end{array}$ & $\begin{array}{l}38 \\
62\end{array}$ & $\begin{array}{l}26 \\
74\end{array}$ & $\begin{array}{l}36 \\
64\end{array}$ & & & 100 & $\begin{array}{l}90 \\
10\end{array}$ & $\begin{array}{l}54 \\
46\end{array}$ & $\begin{array}{l}24 \\
76\end{array}$ & $\begin{array}{l}15 \\
85\end{array}$ \\
\hline $\begin{array}{l}\text { Avec c. (obligatoire) } \\
\text { Avec . (volontaire) }\end{array}$ & & & & & & & & & & & & & & & & & & & & & & \\
\hline $\begin{array}{l}\text { Sans contrainte } \\
\text { Un seul produit }\end{array}$ & $\begin{array}{r}100 \\
0\end{array}$ & $\begin{array}{l}100 \\
1\end{array}$ & $\begin{array}{l}100 \\
0\end{array}$ & $\begin{array}{r}100 \\
0\end{array}$ & $\begin{array}{r}100 \\
1\end{array}$ & 100 & $\begin{array}{r}100 \\
1\end{array}$ & $\begin{array}{r}100 \\
1\end{array}$ & $\begin{array}{r}100 \\
0\end{array}$ & 100 & 100 & 100 & 100 & 100 & $\begin{array}{r}100 \\
?\end{array}$ & $\begin{array}{r}100 \\
5\end{array}$ & $\begin{array}{r}100 \\
13\end{array}$ & $\begin{array}{l}100 \\
18\end{array}$ & $\begin{array}{l}100 \\
27\end{array}$ & $\begin{array}{l}100 \\
36 \\
36\end{array}-3$ & $\begin{array}{r}100 \\
12\end{array}$ & $\begin{array}{c}100 \\
10\end{array}$ \\
\hline Un groupe de produits & 65 & 63 & 52 & 52 & 41 & 33 & 28 & 30 & 19 & 17 & 32 & 37 & 37 & 25 & 34 & 95 & 87 & 82 & 63 & 18 & 12 & 6 \\
\hline $\begin{array}{l}\text { lous les prodults } \\
\text { Etats-Unis (USD) }\end{array}$ & 3779 & 3646 & 3665 & 4204 & 3668 & 3585 & 3292 & 369 & 3141 & $\begin{array}{r}82 \\
3162 \\
\end{array}$ & 3168 & 3096 & 3002 & 3303 & 364 & 3461 & 2979 & 3211 & 3098 & 3168 & 3359 & $\begin{array}{r}85 \\
2929\end{array}$ \\
\hline $\begin{array}{l}\text { Limites } \\
\text { Pas de limites }\end{array}$ & $\begin{array}{r}1 \\
99\end{array}$ & $\begin{array}{r}1 \\
99\end{array}$ & $\begin{array}{r}1 \\
99\end{array}$ & $\begin{array}{r}1 \\
99\end{array}$ & $\begin{array}{r}1 \\
99\end{array}$ & $\begin{array}{c}1 \\
99\end{array}$ & $\begin{array}{r}1 \\
99\end{array}$ & $\begin{array}{r}1 \\
99\end{array}$ & $\begin{array}{r}1 \\
99\end{array}$ & $\begin{array}{r}1 \\
99\end{array}$ & $\begin{array}{r}2 \\
98\end{array}$ & $\begin{array}{r}2 \\
98\end{array}$ & 98 & $\begin{array}{r}2 \\
98\end{array}$ & $\begin{array}{r}2 \\
98\end{array}$ & $\begin{array}{c}2 \\
98\end{array}$ & $\begin{array}{r}2 \\
98\end{array}$ & $\begin{array}{r}1 \\
99\end{array}$ & $\begin{array}{r}2 \\
98\end{array}$ & $\begin{array}{r}1 \\
99\end{array}$ & $\begin{array}{r}1 \\
99\end{array}$ & $\begin{array}{r}1 \\
99\end{array}$ \\
\hline $\begin{array}{l}\text { Fixe } \\
\text { Variable }\end{array}$ & $\begin{array}{l}78 \\
22\end{array}$ & $\begin{array}{l}82 \\
18\end{array}$ & $\begin{array}{l}82 \\
18\end{array}$ & $\begin{array}{l}83 \\
17\end{array}$ & $\begin{array}{l}85 \\
15\end{array}$ & $\begin{array}{l}86 \\
14\end{array}$ & $\begin{array}{r}92 \\
8\end{array}$ & $\begin{array}{c}93 \\
7\end{array}$ & $\begin{array}{r}92 \\
8\end{array}$ & $\begin{array}{r}91 \\
9\end{array}$ & $\begin{array}{r}91 \\
9\end{array}$ & $\begin{array}{r}92 \\
8\end{array}$ & $\begin{array}{r}92 \\
8\end{array}$ & $\begin{array}{c}93 \\
7\end{array}$ & $\begin{array}{r}93 \\
7\end{array}$ & $\begin{array}{r}93 \\
7\end{array}$ & $\begin{array}{c}92 \\
8\end{array}$ & $\begin{array}{r}93 \\
7\end{array}$ & $\begin{array}{r}92 \\
8\end{array}$ & $\begin{array}{r}93 \\
7\end{array}$ & $\begin{array}{r}93 \\
7\end{array}$ & $\begin{array}{l}92 \\
8\end{array}$ \\
\hline $\begin{array}{l}\text { Avec c. (obligatoire) } \\
\text { Avecc c. (volontaire) }\end{array}$ & 22 & 18 & & 17 & 16 & 15 & 9 & 8 & 9 & 10 & 10 & 9 & 9 & 9 & 9 & 9 & 9 & $\stackrel{9}{9}$ & $\begin{array}{l}9 \\
1\end{array}$ & $\begin{array}{l}9 \\
5\end{array}$ & $\begin{array}{l}8 \\
7\end{array}$ & $\begin{array}{l}9 \\
0\end{array}$ \\
\hline $\begin{array}{l}\text { Sans contrainte } \\
\text { Un seul produit }\end{array}$ & 78 & 82 & 81 & 83 & 84 & 85 & 91 & 92 & 91 & 90 & 90 & 91 & 91 & 91 & 91 & 91 & 91 & 91 & 90 & 86 & 85 & 91 \\
\hline $\begin{array}{l}\text { Un groupe de produits } \\
\text { Tous les produits }\end{array}$ & $\begin{array}{l}15 \\
85\end{array}$ & $\begin{array}{l}17 \\
83\end{array}$ & $\begin{array}{l}17 \\
83\end{array}$ & $\begin{array}{l}27 \\
73\end{array}$ & $\begin{array}{l}20 \\
80\end{array}-10$ & $\begin{array}{l}19 \\
81\end{array}$ & $\begin{array}{l}20 \\
80\end{array}$ & $\begin{array}{l}18 \\
82\end{array}$ & $\begin{array}{l}16 \\
84\end{array}$ & $\begin{array}{l}16 \\
84\end{array}$ & $\begin{array}{l}16 \\
84\end{array}$ & $\begin{array}{l}15 \\
85\end{array}$ & $\begin{array}{l}13 \\
87\end{array}$ & $\begin{array}{l}21 \\
79\end{array}$ & $\begin{array}{l}18 \\
82\end{array}$ & $\begin{array}{l}24 \\
76\end{array}$ & $\begin{array}{l}12 \\
88\end{array}$ & $\begin{array}{l}18 \\
82\end{array}$ & $\begin{array}{l}16 \\
84\end{array}$ & $\begin{array}{l}17 \\
83\end{array}$ & $\begin{array}{l}22 \\
78\end{array}$ & $\begin{array}{l}11 \\
89\end{array}$ \\
\hline
\end{tabular}


Tableau annexe 4. Catégorie B2 (PIF) : composition de la catégorie par pays et étiquette, 1986-2007

Part de la catégorie en pourcentage

\begin{tabular}{|c|c|c|c|c|c|c|c|c|c|c|c|c|c|c|c|c|c|c|c|c|c|c|}
\hline OCDE (USD) & $\begin{array}{l}\frac{1986}{6007} \\
6007\end{array}$ & $\begin{array}{l}\frac{1987}{7041} \\
704\end{array}$ & $\begin{array}{l}1988 \\
682\end{array}$ & $\begin{array}{l}1989 \\
5961\end{array}$ & $\frac{1990}{6933}$ & $\begin{array}{l}\frac{1991}{7013} \\
7013\end{array}$ & $\begin{array}{l}\frac{1992}{7763} \\
773\end{array}$ & $\begin{array}{l}1993 \\
8599 \\
\end{array}$ & $\begin{array}{l}1994 \\
7965 \\
\end{array}$ & $\begin{array}{l}1995 \\
7628 \\
\end{array}$ & $\begin{array}{l}\frac{1996}{7503} \\
7503\end{array}$ & $\begin{array}{l}\frac{1997}{7064} \\
7064\end{array}$ & $\begin{array}{l}1998 \\
578\end{array}$ & $\frac{1999}{5728}$ & $\begin{array}{l}2000 \\
5252 \\
5252\end{array}$ & $\frac{2001}{5132}$ & $\frac{2002}{5160}$ & $\frac{2003}{6554}$ & $\begin{array}{l}2004 \\
7739 \\
\end{array}$ & $\begin{array}{l}2005 \\
8451\end{array}$ & $\begin{array}{l}2006 \\
953\end{array}$ & $\begin{array}{r}2007 \\
11786 \\
\end{array}$ \\
\hline $\begin{array}{l}\text { Limites } \\
\text { Pas de limites }\end{array}$ & $\begin{array}{l}20 \\
80\end{array}$ & $\begin{array}{l}18 \\
{ }_{82}\end{array}$ & $\begin{array}{l}18 \\
82\end{array}$ & $\begin{array}{l}21 \\
79 \\
79\end{array}$ & $\begin{array}{r}18 \\
82 \\
87\end{array}$ & $\begin{array}{l}16 \\
84\end{array}$ & $\begin{array}{l}11 \\
89\end{array}$ & $\begin{array}{l}10 \\
90\end{array}$ & $\begin{array}{l}10 \\
90\end{array}$ & $\begin{array}{r}7 \\
93\end{array}$ & $\begin{array}{r}9 \\
91\end{array}$ & $\begin{array}{l}10 \\
90\end{array}$ & $\begin{array}{l}10 \\
90\end{array}$ & $\begin{array}{l}11 \\
89\end{array}$ & $\begin{array}{l}15 \\
85\end{array}$ & $\begin{array}{r}14 \\
86\end{array}$ & $\begin{array}{r}17 \\
83\end{array}$ & $\begin{array}{r}13 \\
87\end{array}$ & $\begin{array}{r}19 \\
81\end{array}$ & $\begin{array}{r}19 \\
81\end{array}$ & $\begin{array}{r}16 \\
84\end{array}$ & $\begin{array}{r}15 \\
85\end{array}$ \\
\hline $\begin{array}{l}\text { Five } \\
\text { Variable }\end{array}$ & $\begin{array}{l}67 \\
33\end{array}$ & $\begin{array}{l}72 \\
28\end{array}$ & $\begin{array}{l}69 \\
31\end{array}$ & $\begin{array}{l}74 \\
26 \\
\end{array}$ & $\begin{array}{l}77 \\
23\end{array}$ & $\begin{array}{l}79 \\
21\end{array}$ & $\begin{array}{l}82 \\
18\end{array}$ & $\begin{array}{l}80 \\
20\end{array}$ & $\begin{array}{l}83 \\
17\end{array}$ & $\begin{array}{l}89 \\
11\end{array}$ & $\begin{array}{l}89 \\
11\end{array}$ & $\begin{array}{l}89 \\
11\end{array}$ & $\begin{array}{l}87 \\
13\end{array}$ & $\begin{array}{l}87 \\
13\end{array}$ & $\begin{array}{l}83 \\
17\end{array}$ & $\begin{array}{l}87 \\
13\end{array}$ & $\begin{array}{l}88 \\
12\end{array}$ & $\begin{array}{l}89 \\
11\end{array}$ & $\begin{array}{l}90 \\
10\end{array}$ & 91 & $\begin{array}{l}90 \\
90 \\
10\end{array}$ & $\begin{array}{l}90 \\
90 \\
10\end{array}$ \\
\hline $\begin{array}{l}\text { Avec c. (obligatiore) } \\
\text { Avec c. (volontaire) }\end{array}$ & $\begin{array}{c}18 \\
2\end{array}$ & $\begin{array}{l}15 \\
3\end{array}$ & $\begin{array}{r}15 \\
3\end{array}$ & $\frac{17}{3}$ & $\begin{array}{r}13 \\
3\end{array}$ & ${ }_{3}^{11}$ & $\begin{array}{l}8 \\
3\end{array}$ & ${ }_{3}^{7}$ & $\begin{array}{l}7 \\
3\end{array}$ & $\begin{array}{l}8 \\
2\end{array}$ & $\stackrel{8}{3}$ & $\begin{array}{l}8 \\
4\end{array}$ & $\frac{9}{4}$ & $\begin{array}{l}9 \\
4\end{array}$ & $\begin{array}{c}10 \\
4\end{array}$ & $\begin{array}{l}9 \\
4\end{array}$ & $\begin{array}{l}9 \\
8\end{array}$ & $\frac{7}{6}$ & $\begin{array}{l}11 \\
12\end{array}$ & $\begin{array}{l}11 \\
13\end{array}$ & $\begin{array}{r}8 \\
10\end{array}$ & 19 \\
\hline 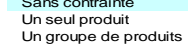 & $\begin{array}{rl}80 & 0 \\
6 & \end{array}$ & $\begin{aligned} 82 \\
0 \\
9 \\
9\end{aligned}$ & $\begin{array}{r}82 \\
0 \\
6\end{array}$ & $\begin{array}{r}79 \\
1 \\
7\end{array}$ & $\begin{array}{r}83 \\
1 \\
8\end{array}$ & $\begin{array}{r}86 \\
1 \\
8\end{array}$ & $\begin{array}{r}89 \\
0 \\
6\end{array}$ & $\begin{array}{r}90 \\
4 \\
8\end{array}$ & $\begin{aligned} 90 \\
0 \\
9\end{aligned}$ & $\begin{array}{r}90 \\
0 \\
7\end{array}$ & $\begin{array}{r}89 \\
1 \\
9\end{array}$ & $\begin{array}{r}88 \\
0 \\
13\end{array}$ & $\begin{array}{r}87 \\
1 \\
13\end{array}$ & $\begin{array}{r}87 \\
3 \\
13\end{array}$ & $\begin{array}{r}86 \\
3 \\
15\end{array}$ & $\begin{array}{r}86 \\
4 \\
14\end{array}$ & $\begin{array}{r}82 \\
5 \\
15\end{array}$ & $\begin{array}{r}87 \\
5 \\
14\end{array}$ & $\begin{array}{r}78 \\
5 \\
12\end{array}$ & $\begin{array}{r}76 \\
5 \\
14\end{array}$ & $\begin{array}{l}82 \\
7 \\
12\end{array}$ & $\begin{array}{c}80 \\
6 \\
11\end{array}$ \\
\hline $\begin{array}{l}\text { Tous les prodoutits } \\
\text { Australie (ISSD) }\end{array}$ & 93 & 91 & 94 & 92 & $\frac{92}{13}$ & 91 & $\begin{array}{l}94 \\
21 \\
21\end{array}$ & $\frac{88}{20}$ & 91 & 93 & $\frac{90}{23}$ & $\frac{87}{29}$ & $\begin{array}{l}86 \\
19 \\
19\end{array}$ & $\begin{array}{l}84 \\
19 \\
19\end{array}$ & $\frac{82}{209}$ & $\begin{array}{r}82 \\
137 \\
137\end{array}$ & 79 & $\frac{81}{102}$ & $\begin{array}{r}83 \\
150 \\
150\end{array}$ & $\begin{array}{r}80 \\
112 \\
112\end{array}$ & $\begin{array}{r}81 \\
107 \\
107\end{array}$ & $\begin{array}{r}82 \\
119 \\
119 \\
-10\end{array}$ \\
\hline $\begin{array}{l}\text { Limites livites } \\
\text { Pas de limites }\end{array}$ & & & $100^{\circ}$ & $100^{\circ}$ & $100^{\circ}-(x)$ & $100^{\circ}-\mathrm{C}$ & $100^{\circ}$ & $100^{\circ}$ & $100^{\circ}$ & $100^{\circ}-(x)$ & $100^{\circ}-(x)$ & $100^{\circ}$ & $100^{\circ}$ & $100^{\circ}$ & $\begin{array}{l}92 \\
8\end{array}$ & $\begin{array}{l}89 \\
11\end{array}$ & $\begin{array}{l}82 \\
18\end{array}$ & 100 & 100 & 100 & 100 & 100 \\
\hline $\begin{array}{l}\text { Fie } \\
\text { Variable }\end{array}$ & & & $100^{\circ}$ & 100 & $100^{\circ}$ & $100^{\circ}-(x)$ & $100^{\circ}$ & $100^{\circ}-(x)$ & $100^{\circ}$ & $100^{\circ}$ & $100^{\circ}$ & 100 & $100^{\circ}$ & $100^{\circ}$ & $100^{\circ}$ & $100^{\circ}$ & $100^{\circ}$ & $100^{\circ}$ & $100^{\circ}$ & $100^{\circ}$ & $100^{\circ}$ & $100^{\circ}$ \\
\hline $\begin{array}{l}\text { Avec c. (obligatiore) } \\
\text { Averc. (volontaire) }\end{array}$ & & & & -1 & 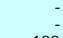 & 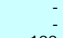 & $-\rho^{-}$ & $=0$ & $-{ }^{-}$ & $m^{-}$ & 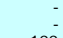 & -1 & & $-\rho^{-}$ & & & 100 & 100 & $100>2>$ & 100 & 100 & $100->$ \\
\hline 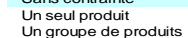 & & & w & 100 & 100 & & & & 100 & & & 100 & 100 & 100 & 100 & 100 & 100 & 100 & 100 & 100 & 100 & 100 \\
\hline $\begin{array}{l}\text { Tous les produits } \\
\text { Canaada (PSD) }\end{array}$ & 466 & 411 & $\frac{100}{457}$ & $\frac{100}{524}$ & $\frac{100}{550}$ & $\frac{100}{535}$ & $\frac{100}{336}$ & $\begin{array}{l}\frac{100}{346} \\
346 \\
-10\end{array}$ & $\frac{100}{273}$ & $\frac{100}{220}$ & $\frac{100}{223}$ & $\frac{100}{212}$ & $\frac{100}{89}$ & $\frac{100}{95}$ & $\begin{array}{r}16 \\
106\end{array}$ & $\frac{22}{79}$ & $\frac{41}{72}$ & $\frac{24}{85}$ & $\frac{19}{106}$ & $\frac{29}{131}$ & $\begin{array}{r}30 \\
193 \\
193\end{array}$ & $\begin{array}{r}30 \\
186 \\
186\end{array}$ \\
\hline $\begin{array}{l}\text { Limites } \\
\text { Pas de limites } \\
\text { Fixe }\end{array}$ & $\begin{array}{l}100 \\
100\end{array}$ & $\begin{array}{l}100 \\
100\end{array}$ & $\begin{array}{l}100 \\
100\end{array}$ & $\begin{array}{l}100 \\
100\end{array}$ & $\begin{array}{l}100 \\
100\end{array}$ & $\begin{array}{l}100^{\circ} \\
100\end{array}$ & $\begin{array}{l}100 \\
100\end{array}$ & $\begin{array}{l}100 \\
100 \\
100\end{array}$ & $\begin{array}{l}100 \\
100\end{array}$ & $\begin{array}{l}100 \\
100\end{array}$ & $\begin{array}{l}100^{\circ} \\
100\end{array}$ & $\begin{array}{l}100 \\
100\end{array}$ & $\begin{array}{l}100 \\
100\end{array}$ & $\begin{array}{l}100 \\
100\end{array}$ & $\begin{array}{l}100^{\circ} \\
100\end{array}$ & $\begin{array}{l}100 \\
100\end{array}$ & $\begin{array}{l}100 \\
100\end{array}$ & $\begin{array}{l}100^{\circ} \\
100\end{array}$ & $\begin{array}{r}96 \\
106 \\
100\end{array}$ & $\begin{array}{r}99 \\
91 \\
100\end{array}$ & $\begin{array}{r}22 \\
78 \\
100\end{array}$ & $\begin{array}{r}21 \\
79 \\
100\end{array}$ \\
\hline $\begin{array}{l}\text { Avec c. (obligatioire) } \\
\text { Avac (volontaire) }\end{array}$ & & & & & & & & & & & & & & & & & & & & & & \\
\hline 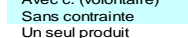 & 100 & 100 & 100 & 100 & 100 & 100 & 100 & 100 & 100 & 100 & $100^{\circ}-x$ & 100 & 100 & 100 & 100 & 100 & $100^{\circ}$ & 100 & $\begin{array}{r}4 \\
96\end{array}$ & 91 & 78 & 79 \\
\hline 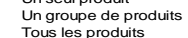 & & & & & & & & $97^{\circ}$ & & & & 足 & & & & & & & & & & \\
\hline $\begin{array}{l}\text { lousles produlits } \\
\text { UE(USD) }\end{array}$ & $\frac{100}{2311}$ & $\begin{array}{l}100 \\
3104\end{array}$ & 2879 & 2045 & 3053 & 2960 & 3990 & 3905 & 3687 & $\begin{array}{l}99 \\
3244\end{array}$ & 3209 & $\frac{100}{3085}$ & $\frac{100}{2471}$ & $\begin{array}{l}100 \\
2446\end{array}$ & $\begin{array}{l}100 \\
1970\end{array}$ & $\begin{array}{l}100 \\
2298 \\
\end{array}$ & $\begin{array}{l}100 \\
2322\end{array}$ & $\begin{array}{l}100 \\
3534\end{array}$ & $\begin{array}{r}100 \\
4642\end{array}$ & $\begin{array}{l}100 \\
4875\end{array}$ & $\begin{array}{l}100 \\
5591\end{array}$ & $\begin{array}{l}100 \\
7185\end{array}$ \\
\hline $\begin{array}{l}\text { Limites } \\
\text { Pas de limites }\end{array}$ & $100^{\circ}$ & $100^{\circ}$ & $100^{\circ}$ & $\begin{array}{l}100 \\
109 \\
09\end{array}$ & $100^{\circ}$ & $100^{\circ}-(x)$ & $100^{\circ}$ & $100^{\circ}$ & $100^{\circ}$ & $100^{\circ}-x$ & $100^{\circ}-x-1$ & 100 & $100^{\circ}$ & $100^{\circ}$ & $100^{\circ}$ & $100^{\circ}$ & $100^{\circ}$ & $100^{\circ}$ & $100^{\circ}-x-10$ & $100^{\circ}$ & $100^{\circ}$ & $100^{\circ}$ \\
\hline $\begin{array}{l}\text { Fixe } \\
\text { Variable } \\
\text { Aviage }\end{array}$ & $\begin{array}{l}87 \\
13\end{array}$ & $\begin{array}{l}87 \\
13\end{array}$ & $\begin{array}{l}81 \\
19\end{array}$ & $\begin{array}{r}99 \\
1 \\
10\end{array}$ & $\begin{array}{r}99 \\
1 \\
10\end{array}$ & $\begin{array}{r}98 \\
2 \\
0\end{array}$ & $\begin{array}{r}96 \\
4 \\
0\end{array}$ & $\begin{array}{r}96 \\
4\end{array}$ & $\begin{array}{r}96 \\
4 \\
2\end{array}$ & $\begin{array}{r}100 \\
0 \\
4\end{array}$ & $\begin{array}{r}100 \\
0 \\
3\end{array}$ & $\begin{array}{r}97 \\
3 \\
3\end{array}$ & $\begin{array}{r}97 \\
3 \\
4\end{array}$ & $\begin{array}{r}99 \\
1 \\
3\end{array}$ & $\begin{array}{r}99 \\
1 \\
5\end{array}$ & $\begin{array}{r}99 \\
1 \\
4\end{array}$ & $\begin{array}{c}98 \\
2 \\
4\end{array}$ & $\begin{array}{r}97 \\
3 \\
3\end{array}$ & $\begin{array}{r}97 \\
3 \\
9\end{array}$ & ${ }_{3}^{97}$ & $\begin{array}{r}94 \\
6\end{array}$ & 8 \\
\hline $\begin{array}{l}\text { Avec c. (volontaire) } \\
\text { Sans contrainte }\end{array}$ & 100 & 100 & 100 & $100^{\circ}-(x)$ & 100 & 100 & $100^{\circ} \mathrm{Y}$ & 99 & 98 & 96 & 97 & & 96 & 97 & & 96 & & & & & & \\
\hline 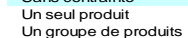 & & & & $\begin{array}{c}0 \\
0 \\
6\end{array}$ & $\begin{array}{r}0 \\
0 \\
7\end{array}$ & $\begin{array}{l}0 \\
0 \\
5\end{array}$ & $\begin{array}{r}0 \\
3 \\
3\end{array}$ & $\begin{array}{c}0 \\
6 \\
6\end{array}$ & $\begin{array}{l}0 \\
0 \\
6\end{array}$ & $\begin{array}{l}0 \\
0 \\
7\end{array}$ & $\begin{array}{l}0 \\
0 \\
7\end{array}$ & $\begin{array}{c}0 \\
12 \\
12\end{array}$ & $\begin{array}{c}0 \\
0 \\
14\end{array}$ & $\begin{array}{c}0 \\
14\end{array}$ & $\begin{array}{r}0 \\
12 \\
12\end{array}$ & $\begin{array}{c}7 \\
10 \\
10\end{array}$ & $\begin{array}{r}9 \\
10 \\
10\end{array}$ & 7 & 7 & $\begin{array}{c}6 \\
9 \\
9\end{array}$ & $\begin{array}{l}5 \\
7\end{array}$ & $\begin{array}{l}4 \\
7\end{array}$ \\
\hline $\begin{array}{l}\text { Tisaus les produlits } \\
\text { Islande (USD) }\end{array}$ & $\frac{99}{7}$ & $\frac{96}{8}$ & $\frac{98}{6}$ & $\begin{array}{l}94 \\
10\end{array}$ & $\frac{93}{5}$ & $\frac{95}{5}$ & $\frac{97}{5}$ & $\frac{94}{4}$ & $\frac{94}{3}$ & $\frac{93}{3}$ & $\frac{93}{3}$ & $\frac{88}{2}$ & $\frac{86}{6}$ & $\frac{86}{4}$ & $\frac{88}{4}$ & $\frac{83}{4}$ & $\frac{81}{4}$ & $\frac{85}{5}$ & $\frac{86}{5}$ & $\frac{84}{7}$ & $\frac{88}{4}$ & $\frac{89}{3}$ \\
\hline $\begin{array}{l}\text { Limites } \\
\text { Pas de limites }\end{array}$ & $\begin{array}{l}10 \\
90\end{array}$ & $\begin{array}{l}17 \\
83\end{array}$ & $\begin{array}{l}21 \\
79\end{array}$ & $\begin{array}{l}10 \\
90 \\
90\end{array}$ & $\begin{array}{l}25 \\
75 \\
\end{array}$ & $\begin{array}{l}17 \\
83\end{array}$ & $\begin{array}{l}11 \\
89\end{array}$ & $\begin{array}{l}13 \\
87\end{array}$ & $\begin{array}{l}20 \\
80\end{array}$ & $\begin{array}{l}18 \\
82\end{array}$ & $\begin{array}{l}34 \\
66\end{array}$ & $\begin{array}{l}24 \\
76\end{array}$ & $\begin{array}{l}13 \\
87\end{array}$ & $\begin{array}{l}23 \\
77\end{array}$ & $\begin{array}{l}23 \\
77\end{array}$ & $\begin{array}{l}21 \\
79\end{array}$ & $\begin{array}{l}14 \\
86\end{array}$ & $\begin{array}{l}11 \\
89\end{array}$ & $\begin{array}{l}13 \\
87\end{array}$ & $\begin{array}{l}11 \\
89\end{array}$ & $\begin{array}{l}28 \\
72\end{array}$ & $100^{\circ}-(x)$ \\
\hline 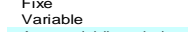 & & & & & & & & & 100 & & & 100 & & & 100 & 100 & 100 & 100 & & 100 & & 100 \\
\hline $\begin{array}{l}\text { Avec c. (obbigatiore) } \\
\text { Avero. (volontaire) } \\
\text { Sons contrainte }\end{array}$ & 100 & 100 & 100 & 100 & 100 & $100^{\circ}$ & 100 & 100 & 100 & $100^{\circ} \mathrm{C}$ & $100^{\circ}$ & 100 & 100 & 100 & 100 & 100 & 100 & $100^{\circ}$ & 100 & 100 & 100 & 100 \\
\hline $\begin{array}{l}\text { Un seul produlut } \\
\text { Un forloupe de produts }\end{array}$ & 89 & 97 & 96 & 96 & 92 & $80^{\circ}$ & 95 & 93 & 89 & $90^{\circ}$ & 96 & 92 & 98 & 99 & 99 & 93 & 93 & 88 & 92 & 95 & 85 & 83 \\
\hline $\begin{array}{l}\text { Tous les profouits } \\
\text { Japon (USD) }\end{array}$ & $\frac{11}{742}$ & $\begin{array}{r}3 \\
916\end{array}$ & $\begin{array}{r}4 \\
1011\end{array}$ & $\begin{array}{r}4 \\
900\end{array}$ & $\frac{8}{870}$ & $\begin{array}{l}200 \\
963\end{array}$ & 1129 & $\frac{7}{1520}$ & $\begin{array}{l}11 \\
1449\end{array}$ & $\begin{array}{r}10 \\
1803\end{array}$ & $\begin{array}{r}4 \\
1360\end{array}$ & $\begin{array}{r}8 \\
1166\end{array}$ & $\begin{array}{r}2 \\
1056\end{array}$ & $\frac{1}{1172}$ & $\frac{1}{970}$ & $\frac{7}{672}$ & $\frac{7}{530}$ & $\frac{12}{552}$ & $\frac{8}{592}$ & $\frac{5}{505}$ & $\frac{15}{444}$ & 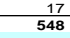 \\
\hline $\begin{array}{l}\text { Limites } \\
\text { Pas de limites } \\
\text { Fixe }\end{array}$ & $\begin{array}{l}100 \\
100\end{array}$ & $\begin{array}{l}100 \\
100\end{array}$ & $\begin{array}{l}100 \\
100\end{array}$ & $\begin{array}{l}100 \\
100\end{array}$ & $\begin{array}{l}100 \\
100\end{array}$ & $\begin{array}{l}100 \\
100\end{array}$ & $\begin{array}{l}100 \\
100\end{array}$ & $\begin{array}{l}100 \\
100 \\
100\end{array}$ & $\begin{array}{l}100 \\
100\end{array}$ & $\begin{array}{l}100 \\
100\end{array}$ & $\begin{array}{l}100 \\
100\end{array}$ & $\begin{array}{l}100 \\
100\end{array}$ & $\begin{array}{l}100 \\
100\end{array}$ & $\begin{array}{l}100 \\
100\end{array}$ & $\begin{array}{l}100 \\
100\end{array}$ & $\begin{array}{l}100 \\
100\end{array}$ & $\begin{array}{l}100 \\
100\end{array}$ & $\begin{array}{l}100 \\
100\end{array}$ & $\begin{array}{l}100 \\
100\end{array}$ & $\begin{array}{l}100 \\
100\end{array}$ & $\begin{array}{l}100 \\
100\end{array}$ & $\begin{array}{l}100 \\
100 \\
100\end{array}$ \\
\hline $\begin{array}{l}\text { Variabl } \\
\text { Avec c. }\end{array}$ & & & & & & & & & & & & & & & & & & & & & & \\
\hline 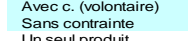 & 100 & 100 & 100 & 100 & $100^{\circ}$ & $100^{\circ}$ & 100 & $100^{\circ}$ & 100 & $100^{\circ}$ & $100^{\circ}$ & 100 & $100^{\circ}$ & $100^{\circ}$ & $100^{\circ}$ & $100^{\circ}$ & 100 & 100 & $100^{\circ}$ & $100^{\circ}$ & $100^{\circ}$ & $100^{\circ}$ \\
\hline 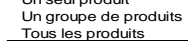 & & & & & & & 100 & 100 & & & 100 & & & & & & 100 & 100 & 100 & 100 & 100 & 100 \\
\hline $\begin{array}{l}\text { lousles profoutis } \\
\text { Corée (USD) }\end{array}$ & 15 & 43 & 105 & 150 & 281 & 344 & 391 & 476 & 632 & 897 & 884 & 784 & 428 & 377 & 345 & 246 & 203 & 162 & 161 & 185 & 215 & 233 \\
\hline $\begin{array}{l}\text { Limites } \\
\text { Pas de limites } \\
\text { Fixe }\end{array}$ & $\begin{array}{l}100 \\
100\end{array}$ & $\begin{array}{l}100 \\
100\end{array}$ & $\begin{array}{l}100 \\
100\end{array}$ & $\begin{array}{l}100 \\
100\end{array}$ & $\begin{array}{l}100 \\
100 \\
100\end{array}$ & $\begin{array}{l}100 \\
100\end{array}$ & $\begin{array}{l}100^{\circ} \\
100\end{array}$ & $\begin{array}{l}100 \\
100\end{array}$ & $\begin{array}{l}100^{\circ} \\
100\end{array}$ & $\begin{array}{l}100^{\circ} \\
100\end{array}$ & $\begin{array}{l}100 \\
100\end{array}$ & $\begin{array}{l}100 \\
100\end{array}$ & $\begin{array}{l}100^{\circ} \\
100\end{array}$ & $\begin{array}{l}100 \\
100\end{array}$ & $\begin{array}{l}100^{\circ} \\
100\end{array}$ & $\begin{array}{l}100^{\circ} \\
100\end{array}$ & $\begin{array}{l}100^{\circ} \\
100\end{array}$ & $\begin{array}{l}100 \\
100\end{array}$ & $\begin{array}{l}100 \\
100\end{array}$ & $\begin{array}{l}100^{\circ} \\
100\end{array}$ & $\begin{array}{l}100^{\circ} \\
100\end{array}$ & $\begin{array}{l}100 \\
100\end{array}$ \\
\hline c c. (obligatoire) & & & & & 0 & 6 & 7 & & & 9 & 9 & 11 & 9 & 19 & 7 & 5 & 8 & 10 & 11 & 13 & 11 & 12 \\
\hline $\begin{array}{l}\text { Avecc. C. (volontaire) } \\
\text { Sans contrainter }\end{array}$ & $100^{\circ}$ & $100^{\circ}$ & $100^{\circ}$ & 100 & $100^{\circ}$ & $94^{-1}$ & 93 & 96 & 97 & $9 i$ & 91 & 89 & $9 i^{\circ}$ & 81 & 93 & 95 & 92 & $90^{\circ}$ & 89 & 87 & $89^{\circ}$ & 88 \\
\hline $\begin{array}{l}\text { Un groupe dep produits } \\
\end{array}$ & $100^{\circ} \mathrm{C}-\mathrm{c}$ & $10^{\circ}$ & $100^{\circ}>$ & $100^{\circ} \mathrm{Cl}$ & 00 & 64 & $\begin{array}{c}7 \\
93\end{array}$ & 4 & $\begin{array}{r}3 \\
97\end{array}$ & 99 & 91 & ${ }_{89}^{11}>>$ & 99 & 19 & 7 & $\begin{array}{r}5 \\
95 \\
95\end{array}$ & 8 & 10 & 10 & ${ }_{87}^{13}$ & 11 & 12 \\
\hline
\end{tabular}




\section{Tableau annexe 4. Catégorie B2 (PIF) : composition de la catégorie par pays et étiquette, 1986-2007 (suite)}

\begin{tabular}{|c|c|c|c|c|c|c|c|c|c|c|c|c|c|c|c|c|c|c|c|c|c|c|}
\hline & \begin{tabular}{|l|l|}
1986 \\
516
\end{tabular} & 1987 & 1988 & $\begin{array}{l}1989 \\
362\end{array}$ & 1990 & 1991 & 1992 & 1993 & $\begin{array}{l}1994 \\
520 \\
50\end{array}$ & 1995 & $\frac{1996}{381}$ & 1997 & 1998 & 1999 & 2000 & 2001 & $\frac{2002}{262}$ & $\begin{array}{ll}2003 \\
500\end{array}$ & 2004 & $\begin{array}{ll}2005 \\
697\end{array}$ & 2006 & 2007 \\
\hline 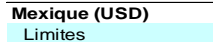 & 516 & 419 & ${ }^{434}$ & 362 & ${ }^{445}$ & 416 & 452 & 765 & 520 & 223 & 381 & 339 & 260 & 398 & 461 & 364 & 262 & 500 & 412 & 697 & 810 & 969 \\
\hline $\begin{array}{l}\text { Limites } \\
\text { Pas de limites }\end{array}$ & 100 & 100 & 100 & 100 & 100 & 100 & $100^{\circ}$ & 100 & 100 & 100 & 100 & 100 & 100 & $100^{\circ}-(x)$ & $100^{\circ}$ & 100 & $100^{\circ}$ & $100^{\circ}$ & $100^{\circ}$ & 100 & $100^{\circ}$ & 100 \\
\hline $\begin{array}{l}\text { Fixe } \\
\text { Variable }\end{array}$ & 100 & 100 & 100 & 100 & 100 & 100 & 100 & 100 & 100 & 100 & $\begin{array}{l}43 \\
57\end{array}$ & $\begin{array}{l}70 \\
30\end{array}$ & $\begin{array}{l}68 \\
32 \\
32\end{array}$ & $\begin{array}{l}54 \\
46\end{array}$ & $\begin{array}{l}59 \\
41\end{array}$ & $\begin{array}{r}95 \\
5\end{array}$ & $\begin{array}{r}93 \\
7\end{array}$ & $\begin{array}{r}94 \\
6\end{array}$ & $\begin{array}{r}94 \\
6\end{array}$ & $\begin{array}{r}98 \\
2\end{array}$ & $\begin{array}{r}94 \\
6\end{array}$ & $\begin{array}{l}89 \\
11\end{array}$ \\
\hline $\begin{array}{l}\text { Avec c. (obbigatoire) } \\
\text { Avec c. (volontaire) }\end{array}$ & & & & & & & & & & & & & & & & & & & & & & \\
\hline Sans contrainte & 100 & 100 & 100 & 100 & 100 & 100 & 100 & 100 & 100 & 100 & 100 & 100 & 100 & 100 & 100 & 100 & 100 & 100 & 100 & 100 & 100 & 100 \\
\hline $\begin{array}{l}\text { Un seut producit } \\
\text { Un groupe de produits }\end{array}$ & 4 & 2 & 2 & 15 & 9 & 15 & 1 & 44 & & & $\begin{array}{l}11 \\
30\end{array}$ & $\begin{array}{r}5 \\
40\end{array}$ & $\begin{array}{l}27 \\
43\end{array}$ & $\begin{array}{l}38 \\
28\end{array}$ & $\begin{array}{l}33 \\
24\end{array}$ & $\begin{array}{r}5 \\
35\end{array}$ & $\begin{array}{r}9 \\
64\end{array}$ & 21 & 20 & 18 & 13 & 11 \\
\hline $\begin{array}{l}\text { Tous les produits } \\
\text { Nelle-zélande (USD) }\end{array}$ & 96 & 98 & $\frac{98}{50}-1 \mathrm{l}$ & 85 & 91 & 85 & 99 & 56 & 100 & 100 & 59 & 54 & 30 & 34 & & 60 & 27 & 79 & 80 & 82 & 87 & 89 \\
\hline $\begin{array}{l}\text { Nelle-Zzelande (USD) } \\
\text { Limites }\end{array}$ & 182 & 229 & 100 & 100 & 20 & & & & & & & & & & & & & & & & & \\
\hline $\begin{array}{l}\text { Pas de limites } \\
\text { Fixe }\end{array}$ & $\begin{array}{l}100 \\
100\end{array}$ & $\begin{array}{l}100 \\
100\end{array}$ & $\begin{array}{l}100 \\
100\end{array}$ & $\begin{array}{l}100 \\
100\end{array}$ & $\begin{array}{l}100 \\
100\end{array}$ & $\begin{array}{l}100 \\
100\end{array}$ & $\begin{array}{l}100 \\
100\end{array}$ & & & & & & & & & & & & & & & \\
\hline $\begin{array}{l}\text { Varaiable } \\
\text { Avec c. (obligatoire) }\end{array}$ & & & & & & & & & & & & & & & & & & & & & & \\
\hline $\begin{array}{l}\text { Avec c. (volontaire) } \\
\text { Sans contrainte }\end{array}$ & 100 & 100 & 100 & 100 & 100 & 100 & $100^{\circ}+2$ & & & & & & & & & & & & & & & \\
\hline $\begin{array}{l}\text { Un seul produit } \\
\text { Un groupe de produits }\end{array}$ & 100 & 100 & 100 & 100 & 100 & 100 & 100 & & & & & & & & & & & & & & & \\
\hline $\begin{array}{l}\text { Tous les prodults } \\
\text { Norvège (USD) }\end{array}$ & 92 & 103 & 78 & 65 & 67 & 62 & 61 & 46 & 41 & 44 & 49 & 61 & 49 & 50 & 47 & 48 & 49 & 50 & 52 & 63 & 67 & 77 \\
\hline $\begin{array}{l}\text { Limites } \\
\text { Pas de limites }\end{array}$ & $100^{\circ}-(x)-1$ & 100 & 100 & 100 & 100 & 100 & 100 & 100 & 100 & 100 & 100 & 100 & 100 & 100 & 100 & 100 & 100 & 100 & 100 & 100 & 100 & 100 \\
\hline $\begin{array}{l}\text { Fixe } \\
\text { Variable }\end{array}$ & $\begin{array}{l}67 \\
33\end{array}$ & $\begin{array}{l}57 \\
43\end{array}$ & $\begin{array}{l}42 \\
58\end{array}$ & $\begin{array}{l}45 \\
55\end{array}$ & $\begin{array}{l}47 \\
53\end{array}$ & $\begin{array}{l}45 \\
55\end{array}$ & $\begin{array}{l}49 \\
51\end{array}$ & $\begin{array}{l}54 \\
46\end{array}$ & 68 & 61 & 71 & 71 & 66 & 72 & $\begin{array}{l}73 \\
27\end{array}$ & 72 & 74 & 80 & 83 & 87 & 88 & 88 \\
\hline 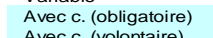 & & & & & & & & & & & & & & & & & & & & & & \\
\hline $\begin{array}{l}\text { Avec c. (Volontare) } \\
\text { Sans contrainte }\end{array}$ & 100 & 100 & 100 & 100 & 100 & 100 & 100 & 100 & 100 & 100 & 100 & 100 & 100 & 100 & 100 & 100 & 100 & $100^{\circ}$ & 100 & 100 & 100 & 100 \\
\hline $\begin{array}{l}\text { Un seul produit } \\
\text { Un groupe de produits }\end{array}$ & 9 & 8 & & 6 & 4 & & 5 & & $\begin{array}{r}5 \\
14\end{array}$ & $\begin{array}{r}5 \\
17\end{array}$ & $\begin{array}{r}5 \\
28\end{array}$ & $\begin{array}{r}4 \\
32\end{array}$ & $\begin{array}{r}5 \\
21\end{array}$ & $\begin{array}{r}5 \\
22\end{array}$ & $\begin{array}{r}5 \\
19\end{array}$ & $\begin{array}{r}5 \\
20\end{array}$ & 19 & 17 & 17 & & 17 & 14 \\
\hline $\begin{array}{l}\text { Tous les produits } \\
\text { Suisse (USD) }\end{array}$ & $\begin{array}{l}91 \\
39 \\
\end{array}$ & 92 & 94 & 94 & 96 & 95 & 95 & 94 & 81 & 78 & 67 & $\frac{64}{50}$ & $\begin{array}{l}74 \\
52\end{array}$ & $\begin{array}{l}73 \\
51 \\
51\end{array}$ & $\begin{array}{r}76 \\
48 \\
48\end{array}$ & $\begin{array}{l}76 \\
52 \\
52\end{array}$ & $\begin{array}{l}81 \\
59\end{array}$ & 83 & 83 & $\begin{array}{r}85 \\
83 \\
3\end{array}$ & 83 & $\frac{86}{91}$ \\
\hline $\begin{array}{l}\text { Limites } \\
\text { Patsimitos }\end{array}$ & 100 & 100 & $100-70$ & 100 & 100 & 100 & 100 & 100 & 100 & 100 & 100 & 100 & 100 & 100 & 100 & 100 & 100 & 100 & 100 & 100 & 100 & 31 \\
\hline $\begin{array}{l}\text { Pas de limites } \\
\text { Fixe }\end{array}$ & 100 & 100 & 100 & 100 & 100 & 100 & 100 & 100 & 100 & 100 & 100 & 100 & 100 & 100 & 100 & 100 & 100 & 100 & 100 & 100 & 100 & 100 \\
\hline $\begin{array}{l}\text { Fixe } \\
\text { Variable }\end{array}$ & 93 & 93 & 93 & $\begin{array}{r}6 \\
94 \\
\end{array}$ & $\begin{array}{r}5 \\
95\end{array}$ & $\begin{array}{r}5 \\
95\end{array}$ & $\begin{array}{r}4 \\
96\end{array}$ & $\begin{array}{r}5 \\
95\end{array}$ & $\begin{array}{r}5 \\
95\end{array}$ & $\begin{array}{r}6 \\
94\end{array}$ & $\begin{array}{l}6 \\
94\end{array}$ & $\begin{array}{l}6 \\
94\end{array}$ & 96 & $\begin{array}{r}6 \\
94\end{array}$ & 93 & $\begin{array}{l}8 \\
92\end{array}$ & 91 & 91 & 91 & 91 & 91 & 91 \\
\hline $\begin{array}{l}\text { Avec c. (obbigatoire) } \\
\text { Avec c. (volontaire) }\end{array}$ & & & & & & & & & & & & & & & & & & & & & & \\
\hline Sans contrainte & 100 & 100 & 100 & 100 & 100 & 100 & 100 & 100 & 100 & 100 & 100 & 100 & 100 & 100 & 100 & 100 & 100 & 100 & 100 & 100 & 100 & 100 \\
\hline $\begin{array}{l}\text { Un seul produit } \\
\text { Un groupe de produits }\end{array}$ & & & & & & & & & & & & & & & & & & & & & & \\
\hline Tous les produits & 100 & 100 & 100 & 100 & 100 & 100 & 100 & 100 & 100 & 100 & 100 & 100 & 100 & 100 & 100 & 100 & 100 & 100 & 100 & 100 & 100 & 100 \\
\hline Turquie (USD) & 5 & 36 & 16 & 43 & 38 & 110 & 37 & 120 & 80 & 50 & 63 & 76 & 70 & 60 & 92 & 113 & 144 & 145 & 238 & 308 & 712 & 775 \\
\hline $\begin{array}{l}\text { Limites } \\
\text { Pas de limites }\end{array}$ & 100 & 100 & 100 & 100 & 100 & 100 & 100 & 100 & 100 & 100 & 100 & 100 & 100 & 100 & 100 & 100 & 100 & 100 & 100 & 100 & 100 & 100 \\
\hline $\begin{array}{l}\text { Fixe } \\
\text { Variable }\end{array}$ & 100 & 100 & 100 & 100 & 100 & 100 & 100 & 100 & 100 & 100 & 100 & 100 & 100 & 100 & 100 & 100 & 100 & 100 & 100 & 100 & 100 & 100 \\
\hline $\begin{array}{l}\text { Avec c. (obligatoire) } \\
\text { Avec c. (volontaire) }\end{array}$ & & & & & & & & & & & & & & & & & & & & & & \\
\hline $\begin{array}{l}\text { Sans contrainte } \\
\text { Shs colvants }\end{array}$ & 100 & 100 & 100 & 100 & 100 & 100 & 100 & 100 & 100 & 100 & 100 & 100 & 100 & 100 & 100 & 100 & 100 & 100 & 100 & 100 & 100 & 100 \\
\hline $\begin{array}{l}\text { Un seul produit } \\
\text { Un groupe de produits }\end{array}$ & 100 & 100 & 100 & 100 & 100 & 100 & 100 & 100 & 100 & 100 & 100 & 100 & 100 & 100 & $\begin{array}{l}21 \\
79\end{array}$ & $\begin{array}{l}30 \\
70\end{array}$ & $\begin{array}{l}32 \\
68\end{array}$ & $\begin{array}{l}31 \\
69\end{array}$ & $\begin{array}{l}28 \\
72\end{array}$ & $\begin{array}{l}43 \\
56\end{array}$ & $\begin{array}{l}51 \\
49\end{array}$ & $\begin{array}{l}52 \\
48\end{array}$ \\
\hline $\begin{array}{l}\text { Tous les produlits } \\
\text { Etats-Unis (USD) }\end{array}$ & 1220 & 1234 & 1246 & 1208 & 1125 & 926 & 749 & 805 & 726 & 555 & 671 & 698 & 588 & 601 & 589 & $\begin{array}{r}0 \\
606\end{array}$ & $\begin{array}{r}0 \\
804\end{array}$ & $\begin{array}{r}0 \\
742\end{array}$ & $\begin{array}{r}0 \\
1301\end{array}$ & $\begin{array}{r}0 \\
1485\end{array}$ & $\begin{array}{r}1 \\
\mathbf{3 6 7}\end{array}$ & 1600 \\
\hline $\begin{array}{l}\text { Limites } \\
\text { Pactom }\end{array}$ & 100 & 100 & 100 & 100 & 100 & 100 & 100 & 100 & 100 & 100 & 100 & 100 & 100 & 100 & 100 & 99 & 100 & 100 & 100 & 100 & 100 & 100 \\
\hline Fie & 12 & $\begin{array}{l}16 \\
84\end{array}$ & 17 & $\begin{array}{l}16 \\
84\end{array}$ & 20 & 22 & 29 & 31 & 32 & 22 & 36 & 46 & 36 & 37 & 36 & 37 & 53 & 49 & 71 & 74 & 72 & 76 \\
\hline & $\begin{array}{l}88 \\
88\end{array}$ & $\begin{array}{l}84 \\
84\end{array}$ & 83 & $\begin{array}{l}84 \\
84\end{array}$ & $\begin{array}{l}80 \\
80\end{array}$ & 78 & 71 & $\begin{array}{l}69 \\
69\end{array}$ & $\begin{array}{l}68 \\
68\end{array}$ & 78 & $\begin{array}{l}64 \\
64\end{array}$ & $\begin{array}{l}54 \\
54\end{array}$ & $\begin{array}{l}64 \\
64\end{array}$ & 63 & $\begin{array}{l}64 \\
64\end{array}$ & $\begin{array}{l}63 \\
64\end{array}$ & $\begin{array}{l}4 f \\
47\end{array}$ & $\begin{array}{l}51 \\
51\end{array}$ & $\begin{array}{l}299 \\
29\end{array}$ & $\begin{array}{l}26 \\
26\end{array}$ & $\begin{array}{l}28 \\
28\end{array}$ & $\begin{array}{l}24 \\
24\end{array}$ \\
\hline $\begin{array}{l}\text { Avec c. (volontaire) } \\
\text { Sans contrainte }\end{array}$ & 12 & 16 & 17 & 16 & 20 & 22 & 29 & 31 & 32 & 22 & 36 & $\begin{array}{r}39 \\
7\end{array}$ & $\begin{array}{r}35 \\
1\end{array}$ & $\begin{array}{r}36 \\
1\end{array}$ & $\begin{array}{r}35 \\
1\end{array}$ & $\begin{array}{r}36 \\
0\end{array}$ & $\begin{array}{r}53 \\
0\end{array}$ & 49 & $\begin{array}{r}71 \\
0\end{array}$ & $\begin{array}{l}74 \\
0\end{array}$ & $\begin{array}{l}67 \\
6\end{array}$ & 76 \\
\hline $\begin{array}{l}\text { Un seul produit } \\
\text { Un groupe de produits }\end{array}$ & 12 & 16 & 17 & 16 & 20 & 22 & 29 & 31 & 32 & 22 & 17 & 20 & 8 & 14 & 12 & $\begin{array}{l}1 \\
9\end{array}$ & 13 & $\begin{array}{r}0 \\
27\end{array}$ & 17 & 22 & 18 & 17 \\
\hline Tous les produits & 88 & & & & & & & & 68 & 78 & 83 & 80 & 92 & 86 & 88 & 90 & 87 & 73 & 83 & 78 & 82 & 83 \\
\hline
\end{tabular}


Tableau annexe 5. Catégorie B3 (PIS) : composition de la catégorie par pays et étiquette, 1986-2007

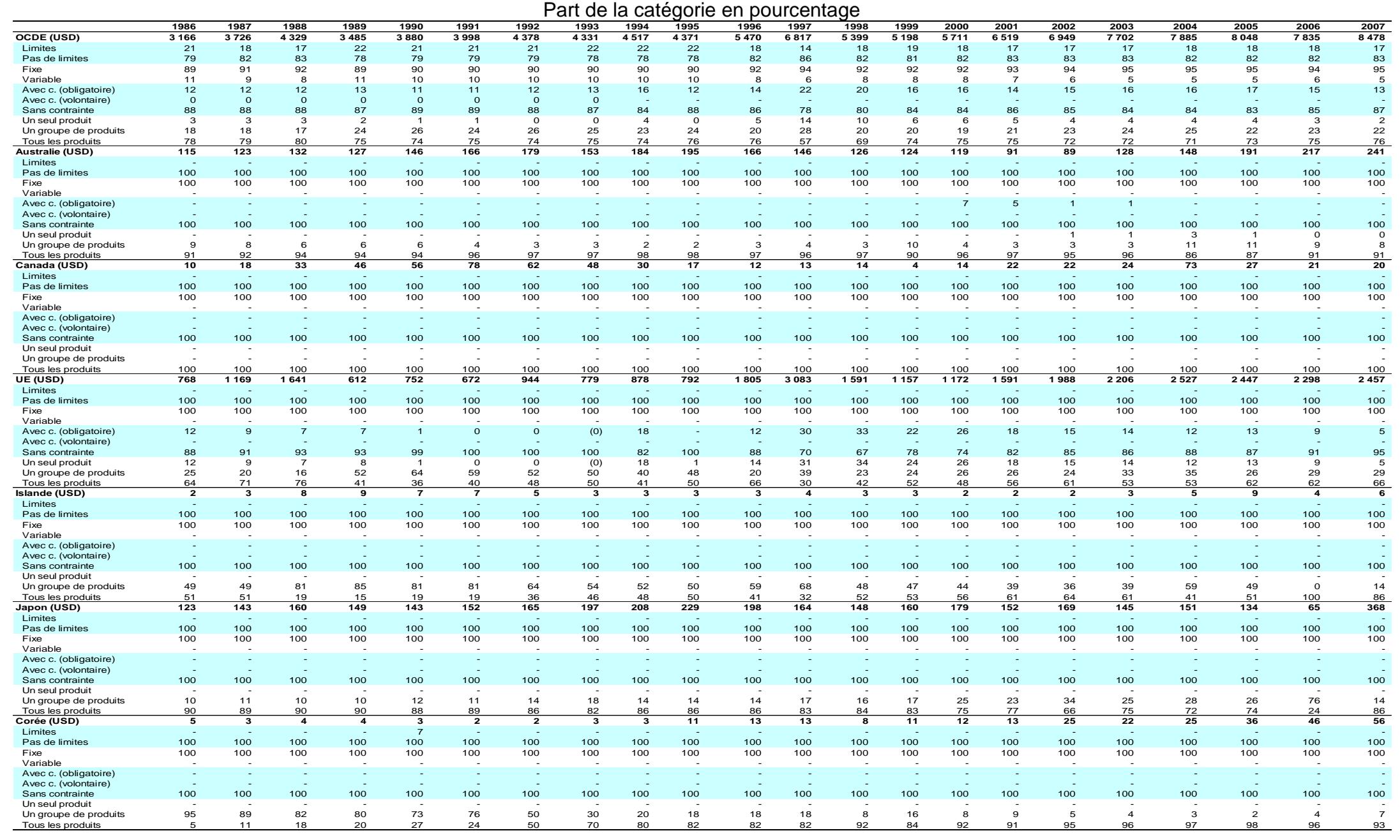

ANALYSE DE LA COMPOSITION DU SOUTIEN AUX PRODUCTEURS : NOUVEAUX OUTILS ET MÉTHODES ๑ OECD 2010 
Tableau annexe 5. Catégorie B3 (PIS) : composition de la catégorie par pays et étiquette, 1986-2007 (suite)

\begin{tabular}{|c|c|c|c|c|c|c|c|c|c|c|c|c|c|c|c|c|c|c|c|c|c|c|}
\hline Mexique (USD) & $\begin{array}{r}1986 \\
40\end{array}$ & $\frac{1987}{36}$ & $\frac{1988}{32}$ & $\frac{1989}{43}$ & $\frac{1990}{110}$ & $\frac{1991}{121}$ & $\frac{1992}{171}$ & $\begin{array}{r}1993 \\
166\end{array}$ & $\begin{aligned} 1994 \\
180\end{aligned}$ & $\begin{array}{r}1995 \\
98\end{array}$ & $\begin{array}{l}1996 \\
140\end{array}$ & $\frac{1997}{171}$ & $\begin{array}{l}1998 \\
127 \\
\end{array}$ & $\frac{1999}{160}$ & $\frac{2000}{187}$ & $\frac{2001}{228}$ & $\frac{2002}{309}$ & $\frac{2003}{399}$ & $\frac{2004}{305}$ & $\frac{2005}{337}$ & $\frac{2006}{364}$ & $\frac{2007}{480}$ \\
\hline $\begin{array}{l}\text { Limites } \\
\text { Pam de limites }\end{array}$ & 100 & 100 & 100 & 100 & 100 & 100 & 100 & 100 & 100 & 100 & 100 & 100 & 100 & 100 & 100 & 100 & 100 & 100 & 100 & 100 & 100 & 10 \\
\hline $\begin{array}{l}\text { Fixe } \\
\text { Variable }\end{array}$ & 100 & 100 & 100 & 100 & 100 & 100 & 100 & 100 & 100 & 100 & 100 & 100 & 100 & 100 & 100 & 100 & 100 & 100 & 100 & 100 & 100 & 100 \\
\hline $\begin{array}{l}\text { Avec C. (obligatoire) } \\
\text { Avec. (bigntiris) }\end{array}$ & - & - & - & - & - & - & - & - & - & - & - & - & - & - & . & - & - & - & - & - & - & \\
\hline $\begin{array}{l}\text { Avec c. c. volontataire) } \\
\text { Sans contrainte }\end{array}$ & 100 & 100 & 100 & 100 & 100 & 100 & 100 & 100 & 100 & 100 & 100 & 100 & 100 & 100 & 100 & 100 & 100 & 100 & 100 & 100 & 100 & 100 \\
\hline & 3 & 2 & 3 & 2 & 1 & 3 & 2 & 21 & - & 13 & 3 & 4 & 6 & 10 & 5 & 6 & $\begin{array}{r}0 \\
0\end{array}$ & 10 & 6 & $\begin{array}{r}5 \\
78\end{array}$ & 4 & $\begin{array}{l}10 \\
56\end{array}$ \\
\hline $\begin{array}{l}\text { Un groupe de produits } \\
\text { Tous les produits }\end{array}$ & $\begin{array}{l}44 \\
53\end{array}$ & $\begin{array}{l}29 \\
69 \\
\end{array} \mathrm{~V}$ & $\begin{array}{l}27 \\
70\end{array}$ & $\begin{array}{l}25 \\
73 \\
\end{array}$ & 87 & $\begin{array}{l}13 \\
84\end{array}$ & 76 & $\begin{array}{l}21 \\
79 \\
\end{array}$ & $\begin{array}{l}17 \\
83\end{array}$ & 87 & $\begin{array}{l}40 \\
57\end{array}$ & $\begin{array}{l}49 \\
48\end{array}$ & $\begin{array}{l}12 \\
22\end{array}$ & $\begin{array}{l}66 \\
24\end{array}$ & $\begin{array}{l}70 \\
26\end{array}$ & $\begin{array}{l}78 \\
16\end{array}$ & $\begin{array}{l}89 \\
11\end{array}$ & $\begin{array}{l}64 \\
26 \\
26\end{array}$ & $\begin{array}{l}69 \\
24\end{array}$ & 78 & $\begin{array}{l}70 \\
26\end{array}$ & $\begin{array}{l}56 \\
33\end{array}$ \\
\hline $\begin{array}{l}\text { Ioususesproutis } \\
\text { Nelle-zélande (USD) }\end{array}$ & 21 & 24 & 25 & 24 & 19 & 17 & 16 & 18 & 34 & 38 & 38 & 38 & 30 & 30 & 26 & 30 & $\frac{11}{23}$ & $\frac{26}{31}$ & $\frac{24}{40}$ & 38 & $\frac{26}{36}$ & 44 \\
\hline $\begin{array}{l}\text { Limitises } \\
\text { Pas de limites }\end{array}$ & 100 & 100 & 100 & 100 & 100 & 100 & 100 & 100 & 100 & 100 & 100 & 100 & 100 & 100 & 100 & 100 & $100^{\circ}$ & 100 & 100 & 100 & 100 & 100 \\
\hline Fixe & 100 & 100 & 100 & 100 & 100 & 100 & 100 & 100 & 100 & 100 & 100 & 100 & 100 & 100 & 100 & 100 & 100 & 100 & 100 & 100 & 100 & 100 \\
\hline $\begin{array}{l}\text { Avec C. (obligatoire) } \\
\text { Avec c. (volontaire) }\end{array}$ & & & & & & & & & & & & & & & & & & & & & & \\
\hline $\begin{array}{l}\text { Sans contrainte } \\
\text { Salte }\end{array}$ & 100 & 100 & 100 & 100 & 100 & 100 & 100 & 100 & 100 & 100 & 100 & 100 & 100 & 100 & 100 & 100 & 100 & 100 & 100 & 100 & 100 & 100 \\
\hline Un groupe de produits & 75 & 80 & 84 & 92 & 83 & 89 & 95 & 94 & 57 & 65 & 61 & 60 & 60 & 65 & 68 & 69 & 57 & 61 & 54 & 60 & 58 & 53 \\
\hline $\begin{array}{l}\text { Tous les produits } \\
\text { Norvège (USD) }\end{array}$ & $\frac{25}{10}$ & $\frac{20}{11}$ & & $\begin{array}{r}8 \\
11\end{array}$ & 12 & 12 & $\frac{5}{13}$ & $\begin{array}{l}6 \\
11\end{array}$ & 11 & 11 & 11 & $\frac{40}{10}$ & $\frac{40}{8}$ & $\frac{35}{9}$ & $\frac{32}{8}$ & 7 & $\frac{43}{8}$ & $\frac{39}{11}$ & $\frac{46}{12}$ & $\frac{40}{13}$ & $\frac{42}{12}$ & $\frac{47}{12}$ \\
\hline $\begin{array}{l}\text { Limites } \\
\text { Pas de limites }\end{array}$ & 100 & 100 & 100 & 100 & 100 & 100 & 100 & 100 & 100 & 100 & 100 & 100 & 100 & 100 & 100 & 100 & 100 & 100 & 100 & 100 & 100 & 100 \\
\hline $\begin{array}{l}\text { Fixe } \\
\text { Varizble }\end{array}$ & 100 & 100 & 100 & 100 & 100 & 100 & 100 & 100 & 100 & 100 & 100 & 100 & 100 & 100 & 100 & 100 & 100 & 100 & 100 & 100 & 100 & 100 \\
\hline $\begin{array}{l}\text { Avec c. (obligatoire) } \\
\text { Avec c. (volontaire) }\end{array}$ & 1 & 2 & 3 & 5 & 12 & & 6 & & & & & & & & & & & & & & & \\
\hline $\begin{array}{l}\text { Sans contrainte } \\
\text { Uns seluroduit }\end{array}$ & 99 & 98 & 97 & 95 & 88 & 94 & 94 & 93 & 100 & 100 & 100 & 100 & 100 & 100 & 100 & 100 & 100 & 100 & 100 & 100 & 100 & 100 \\
\hline Un groupe de produits & 100 & 100 & 100 & 100 & 100 & 100 & 100 & 100 & 100 & 100 & 100 & 100 & 100 & 100 & 100 & 100 & 100 & 100 & 100 & 100 & 100 & 100 \\
\hline $\begin{array}{l}\text { Toous les produits } \\
\text { Suisse (USD) }\end{array}$ & 20 & 24 & 25 & 22 & 26 & 26 & 15 & 14 & 16 & 18 & 17 & 21 & 18 & 10 & 10 & 24 & 31 & 11 & 12 & 12 & 10 & 23 \\
\hline $\begin{array}{l}\text { Limitise } \\
\text { Pas de limites }\end{array}$ & $100^{\circ}$ & 100 & 100 & $100^{\circ}$ & 100 & 100 & 100 & 100 & 100 & 100 & 100 & 100 & 100 & 100 & 100 & 100 & 100 & 100 & 100 & 100 & 100 & 100 \\
\hline $\begin{array}{l}\text { Fixe } \\
\text { Varizble }\end{array}$ & 100 & 100 & 100 & 100 & 100 & 100 & 100 & 100 & 100 & 100 & 100 & 100 & 100 & 100 & 100 & 100 & 100 & 100 & 100 & 100 & 100 & 100 \\
\hline $\begin{array}{l}\text { Avec C. (obligatoire) } \\
\text { Avec (vilontair) }\end{array}$ & - & $\cdots$ & - & - & : & & - & - & - & - & - & - & - & - & - & - & - & - & - & - & - & \\
\hline $\begin{array}{l}\text { Avecc. C. Volontare) } \\
\text { Sans contrainte }\end{array}$ & $100^{\circ}-(x)-3$ & 100 & 100 & 100 & $100^{\circ}-(x)$ & 100 & 100 & 100 & 100 & 100 & 100 & 100 & 100 & 100 & 100 & 100 & 100 & 100 & 100 & 100 & $100^{\circ}$ & 100 \\
\hline $\begin{array}{l}\text { Un seul produlit } \\
\text { Un groupe de produits }\end{array}$ & 43 & 44 & 43 & 43 & 44 & 44 & 2 & 2 & 4 & 4 & 3 & 33 & 26 & 29 & 46 & 75 & 81 & 39 & 41 & 38 & 27 & 67 \\
\hline $\begin{array}{l}\text { Tous les produits } \\
\text { Turquie (USD) }\end{array}$ & $\frac{57}{14}$ & $\frac{56}{17}$ & $\frac{57}{16}$ & $\frac{57}{26}$ & $\frac{56}{26}$ & $\frac{56}{46}$ & $\frac{98}{27}$ & $\frac{98}{20}$ & $\frac{96}{11}$ & $\frac{96}{7}$ & $\frac{97}{9}$ & $\frac{67}{13}$ & $\frac{74}{10}$ & 7 & $\frac{54}{8}$ & $\frac{25}{8}$ & 9 & $\frac{61}{10}$ & $\frac{59}{12}$ & $\frac{62}{36}$ & $\frac{73}{43}$ & $\frac{33}{44}$ \\
\hline $\begin{array}{l}\text { Limites } \\
\text { Pas de limites }\end{array}$ & 100 & 100 & 100 & 100 & 100 & 100 & 100 & 100 & 100 & 100 & 100 & 100 & 100 & 100 & 100 & 100 & 100 & 100 & 100 & 100 & 100 & 100 \\
\hline $\begin{array}{l}\text { Fixe } \\
\text { Fixe }\end{array}$ & 100 & 100 & 100 & 100 & 100 & 100 & 100 & 100 & 100 & 100 & 100 & 100 & 100 & 100 & 100 & 100 & 100 & 100 & 100 & 100 & 100 & 100 \\
\hline $\begin{array}{l}\text { Avec c. (obligatoire) } \\
\text { Avecc (volontaire) }\end{array}$ & - & - & - & & - & & & - & - & & - & & - & & - & - & - & - & - & - & - & \\
\hline $\begin{array}{l}\text { Avec c. (vVlontaire } \\
\text { Sans contrainte }\end{array}$ & 100 & 100 & 100 & 100 & 100 & 100 & 100 & 100 & 100 & 100 & 100 & 100 & 100 & 100 & 100 & 100 & 100 & 100 & 100 & 100 & $100^{\circ}$ & 100 \\
\hline $\begin{array}{l}\text { Un suel produlit } \\
\text { Un groupe de produits }\end{array}$ & $\begin{array}{l}52 \\
48\end{array}$ & $\begin{array}{l}50 \\
50\end{array}$ & $\begin{array}{l}46 \\
54\end{array}$ & $\begin{array}{l}59 \\
41\end{array}$ & $\begin{array}{l}78 \\
22\end{array}$ & $\begin{array}{l}54 \\
46\end{array}$ & $\begin{array}{l}34 \\
66\end{array}$ & $\begin{array}{l}52 \\
48\end{array}$ & $\begin{array}{l}42 \\
58\end{array}$ & $100^{\circ}$ & $100^{\circ}$ & $\begin{array}{l}36 \\
64\end{array}$ & $\begin{array}{l}16 \\
84\end{array}$ & $\begin{array}{l}13 \\
87\end{array}$ & $\begin{array}{r}3 \\
97\end{array}$ & $100^{\circ}$ & $100^{\circ}$ & $100^{\circ}$ & $100^{\circ}$ & $100^{\circ}$ & $100^{\circ}-(x)-1$ & $100^{\circ}$ \\
\hline $\begin{array}{l}\text { Tous les produits } \\
\text { Etats-Unis (USD) }\end{array}$ & 2027 & 2140 & 2227 & 2404 & 2556 & 2632 & 2716 & 2850 & 2898 & 2900 & 3008 & 3082 & 3246 & 3458 & 3915 & 4268 & 4170 & 4645 & 4575 & 4770 & 4717 & 4727 \\
\hline Limites & 32 & 32 & 32 & 32 & 31 & 31 & 34 & 33 & 34 & 33 & 32 & 32 & 31 & 29 & 27 & 26 & 29 & 29 & 31 & 31 & 31 & 31 \\
\hline Pas de limites & 68 & 68 & 68 & 68 & 69 & 69 & 66 & 67 & 66 & 67 & 68 & 68 & 69 & 71 & 73 & 74 & 71 & 71 & 69 & 69 & 69 & 69 \\
\hline Fixe & 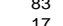 & $\begin{array}{l}85 \\
{ }_{15}\end{array}$ & $\begin{array}{l}86 \\
{ }_{14}^{6}\end{array}$ & 要 & $\begin{array}{l}86 \\
14\end{array}$ & 栗6 & 每5 & 列 86 & $\begin{array}{l}85 \\
85\end{array}$ & 列 85 & 列 86 & 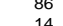 & (87 & $\begin{array}{l}88 \\
12\end{array}$ & 要9 & $\begin{array}{r}90 \\
10\end{array}$ & 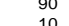 & 91 & 91 & 91 & 90 & 91 \\
\hline Ave & 15 & $\begin{array}{l}15 \\
16\end{array}$ & $\begin{array}{l}P_{14}^{14} \\
17\end{array}$ & $\begin{array}{l}15 \\
17\end{array}$ & $\begin{array}{l}14 \\
16\end{array}$ & $\begin{array}{l}14 \\
17\end{array}$ & $\begin{array}{l}15 \\
19\end{array}$ & 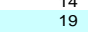 & $\begin{array}{l}15 \\
19\end{array}$ & 18 & $\begin{array}{l}7_{18}^{4} \\
18\end{array}$ & $\begin{array}{l}l_{14}^{14} \\
18\end{array}$ & $\begin{array}{l}13 \\
18\end{array}$ & $\begin{array}{l}12 \\
17\end{array}$ & $\begin{array}{l}11 \\
15\end{array}$ & $\begin{array}{l}10 \\
16\end{array}$ & $\begin{array}{l}10 \\
18\end{array}$ & 20 & 22 & $\begin{array}{l}9 \\
22\end{array}$ & $\begin{array}{l}10 \\
21\end{array}$ & $\begin{array}{r}9 \\
21\end{array}$ \\
\hline $\begin{array}{l}\text { Avec c. c. Volontail } \\
\text { Sans contrainte }\end{array}$ & 85 & 84 & 83 & 83 & 84 & 83 & 81 & 81 & 81 & 82 & 82 & 82 & 82 & 83 & 85 & 84 & 82 & 80 & 78 & 78 & 79 & 79 \\
\hline $\begin{array}{l}\text { Un sued produti } \\
\text { Un groupe de produts }\end{array}$ & 15 & 16 & 17 & 17 & 16 & 17 & 19 & 19 & 19 & 18 & 18 & 17 & 17 & 16 & 15 & 15 & 16 & $\begin{array}{l}0 \\
16\end{array}$ & 17 & 16 & 15 & 16 \\
\hline
\end{tabular}


Tableau annexe 6. Catégorie C (PC) : composition de la catégorie par pays et étiquette, 1986-2007

$$
\text { Part de la catégorie en pourcentage }
$$

\begin{tabular}{|c|c|c|c|c|c|c|c|c|c|c|c|c|c|c|c|c|c|c|c|c|c|c|}
\hline CDE (USD) & $\begin{array}{l}1988 \\
1994\end{array}$ & 19987 & $\begin{array}{l}1988 \\
826 \\
326\end{array}$ & $\begin{array}{l}1989 \\
1967\end{array}$ & 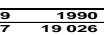 & $\begin{array}{l}\frac{1991}{9355} \\
135\end{array}$ & 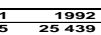 & 1993 & $\begin{array}{l}1994 \\
6688 \\
6\end{array}$ & 995 & $\begin{array}{l}\frac{1996}{2754} \\
2754\end{array}$ & 1997 & $\begin{array}{l}1998 \\
0580 \\
0.58\end{array}$ & 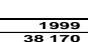 & $\begin{array}{l}20000 \\
38104\end{array}$ & $\begin{array}{l}2001 \\
4057\end{array}$ & $\begin{array}{l}20002 \\
43892\end{array}$ & $\begin{array}{l}20033 \\
50650\end{array}$ & $\begin{array}{l}2004 \\
55067\end{array}$ & $\begin{array}{l}20005 \\
38100\end{array}$ & \begin{tabular}{|l|l|l|l|}
2006 \\
291982
\end{tabular} & $\begin{array}{l}2007 \\
27728 \\
\end{array}$ \\
\hline 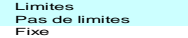 & $\begin{array}{l}60 \\
40 \\
28 \\
28\end{array}$ & $\begin{array}{l}63 \\
37 \\
37\end{array}$ & 就 & $\begin{array}{r}39 \\
61 \\
61\end{array}$ & $\begin{array}{l}0.26 \\
59 \\
59\end{array}$ & $\begin{array}{r}355 \\
65 \\
65\end{array}$ & $\begin{array}{l}634 \\
36 \\
36\end{array}$ & $\begin{array}{l}890 \\
31 \\
31\end{array}$ & 年 & 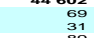 & $\begin{array}{l}2754 \\
36 \\
34 \\
34\end{array}$ & $\begin{array}{l}781 \\
67 \\
33 \\
33\end{array}$ & $\begin{array}{l}0.58 \\
62 \\
38 \\
38\end{array}$ & $\begin{array}{l}3810 \\
\substack{38 \\
40} \\
40\end{array}$ & $\begin{array}{l}38 \text { 104 } \\
355 \\
35\end{array}$ & 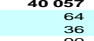 & $\begin{array}{l}4389 \\
63 \\
37 \\
37\end{array}$ & $\begin{array}{l}50650 \\
56 \\
34 \\
34\end{array}$ & 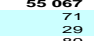 & $\begin{array}{l}38108 \\
38 \\
42 \\
42\end{array}$ & $\begin{array}{l}2982 \\
44 \\
56\end{array}$ & 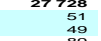 \\
\hline 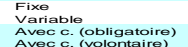 & $\frac{28}{72}$ & $\begin{array}{l}31 \\
69 \\
6 \\
9\end{array}$ & $\begin{array}{l}52 \\
48 \\
29 \\
29\end{array}$ & 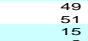 & $\begin{array}{l}48 \\
\frac{12}{6} \\
56\end{array}$ & $\begin{array}{l}48 \\
57 \\
57\end{array}$ & 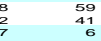 & $\begin{array}{l}724 \\
26 \\
17 \\
71\end{array}$ & $\begin{array}{l}78 \\
72 \\
10 \\
10\end{array}$ & $\begin{array}{l}89 \\
11 \\
9\end{array}$ & $\frac{93}{8}$ & $\begin{array}{c}94 \\
6 \\
5 \\
5\end{array}$ & 91 & $\begin{array}{l}89 \\
14 \\
14\end{array}$ & $\begin{array}{l}90 \\
\substack{9 \\
16} \\
16\end{array}$ & 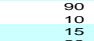 & $\begin{array}{l}85 \\
18 \\
18 \\
\end{array}$ & 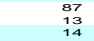 & $\begin{array}{l}89 \\
44 \\
44\end{array}$ & $\begin{array}{l}89 \\
11 \\
35\end{array}$ & $\begin{array}{l}83 \\
12 \\
20\end{array}$ & $\begin{array}{c}89 \\
19 \\
19\end{array}$ \\
\hline 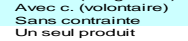 & $\begin{array}{l}85 \\
73 \\
73\end{array}$ & $\begin{array}{c}85 \\
79 \\
79\end{array}$ & $\begin{array}{c}65 \\
59 \\
59\end{array}$ & $\begin{array}{l}75 \\
75 \\
62\end{array}$ & 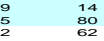 & $\begin{array}{c}13 \\
\text { so } \\
55\end{array}$ & $\begin{array}{c}18 \\
76 \\
50\end{array}$ & 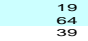 & $\begin{array}{l}22 \\
69 \\
38 \\
38\end{array}$ & $\begin{array}{l}27 \\
\text { 264 } \\
299 \\
29\end{array}$ & 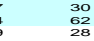 & $\begin{array}{l}32 \\
62 \\
26 \\
26 \\
2\end{array}$ & $\begin{array}{c}29 \\
29 \\
27\end{array}$ & $\begin{array}{l}29 \\
\frac{29}{29} \\
29 \\
0\end{array}$ & $\begin{array}{l}29 \\
55 \\
29 \\
29\end{array}$ & 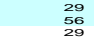 & $\begin{array}{l}28 \\
\begin{array}{r}28 \\
37\end{array} \\
37\end{array}$ & 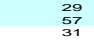 & $\begin{array}{l}\frac{32}{22} \\
31 \\
31\end{array}$ & $\begin{array}{l}\frac{38}{28} \\
25 \\
25\end{array}$ & $\begin{array}{l}48 \\
32 \\
25 \\
25\end{array}$ & $\begin{array}{l}48 \\
33 \\
23 \\
23\end{array}$ \\
\hline 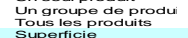 & $\begin{array}{l}16 \\
11 \\
72\end{array}$ & & $\begin{array}{ll}33 \\
3 \\
64 \\
6\end{array}$ & $\begin{array}{l}26 \\
72 \\
158 \\
158\end{array}$ & $\begin{array}{l}22 \\
26 \\
16 \\
16\end{array}$ & $\begin{array}{l}32 \\
33 \\
50 \\
50\end{array}$ & $\begin{array}{l}39 \\
30 \\
61 \\
61\end{array}$ & $\begin{array}{c}53 \\
57 \\
72 \\
7\end{array}$ & $\begin{array}{l}54 \\
50 \\
60\end{array}$ & $\begin{array}{l}59 \\
11 \\
10 \\
68\end{array}$ & $\begin{array}{l}\underbrace{}_{12} \\
12 \\
168\end{array}$ & $\begin{array}{l}59 \\
514 \\
67 \\
6.5\end{array}$ & $\begin{array}{l}59 \\
14 \\
68 \\
68\end{array}$ & $\begin{array}{l}55 \\
56 \\
66 \\
67\end{array}$ & $\begin{array}{l}79 \\
24 \\
27 \\
73\end{array}$ & $\begin{array}{l}\frac{14}{24} \\
27 \\
73\end{array}$ & 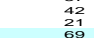 & $\begin{array}{l}44 \\
25 \\
25 \\
65\end{array}$ & 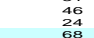 & $\begin{array}{l}\substack{41 \\
35 \\
71} \\
31\end{array}$ & $\begin{array}{l}27 \\
48 \\
70\end{array}$ & $\begin{array}{l}\substack{29 \\
48 \\
68} \\
68\end{array}$ \\
\hline 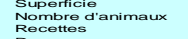 & $\begin{array}{l}72 \\
14 \\
4\end{array}$ & $\begin{array}{r}71 \\
79 \\
4\end{array}$ & 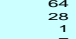 & 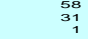 & $\begin{array}{c}56 \\
33 \\
3 \\
3\end{array}$ & $\begin{array}{c}50 \\
35 \\
8\end{array}$ & $\begin{array}{c}61 \\
29 \\
4\end{array}$ & $\begin{array}{l}\frac{72}{24} \\
2 \\
2\end{array}$ & $\begin{array}{c}69 \\
27 \\
2\end{array}$ & $\begin{array}{c}68 \\
28 \\
1 \\
1\end{array}$ & $\begin{array}{c}68 \\
30 \\
1 \\
1\end{array}$ & 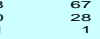 & $\begin{array}{l}68 \\
27 \\
11\end{array}$ & $\begin{array}{c}67 \\
26 \\
11 \\
1\end{array}$ & 隽3 & $\begin{array}{c}73 \\
73 \\
1 \\
1 \\
1\end{array}$ & 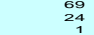 & $\begin{array}{c}67 \\
23 \\
2 \\
2\end{array}$ & $\begin{array}{c}68 \\
25 \\
21 \\
11\end{array}$ & $\begin{array}{l}71 \\
718 \\
2\end{array}$ & $\begin{array}{c}70 \\
16 \\
2\end{array}$ & $\begin{array}{l}68 \\
18 \\
3 \\
3\end{array}$ \\
\hline 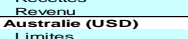 & & & & & & & & & & & & & & & & & & & & & & $\frac{10}{50}$ \\
\hline $\begin{array}{l}\text { Limites limites } \\
\text { Pas die dimites }\end{array}$ & $\begin{array}{l}100 \\
100\end{array}$ & $\begin{array}{l}100 \\
100\end{array}$ & ${ }_{100}^{100}$ & $\begin{array}{l}100 \\
100\end{array}$ & ${ }_{100}^{100}$ & 100 & 100 & $\begin{array}{l}100 \\
100\end{array}$ & 100 & 100 & $\begin{array}{l}100 \\
100\end{array}$ & $\begin{array}{l}100 \\
100\end{array}$ & $\begin{array}{l}100 \\
100\end{array}$ & $\begin{array}{l}100 \\
100\end{array}$ & $\begin{array}{l}100 \\
100\end{array}$ & $\begin{array}{l}100 \\
100\end{array}$ & $\begin{array}{l}100 \\
100\end{array}$ & $\begin{array}{l}100 \\
100\end{array}$ & $\begin{array}{l}100 \\
100\end{array}$ & $\begin{array}{c}100 \\
100\end{array}$ & $\begin{array}{l}100 \\
100\end{array}$ & 100 \\
\hline 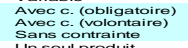 & $100^{\circ}$ & $100^{\circ}$ & $100^{\circ}$ & $100^{\circ}-(x)$ & $100^{\circ}$ & $100^{\circ}-(x)$ & $100^{\circ}-(x)$ & 100 & $100^{\circ}$ & $100^{\circ}$ & $100^{\circ}$ & $100^{\circ}$ & 100 & 100 & $100^{\circ}$ & 100 & $100^{\circ}$ & $100^{\circ}$ & $100^{\circ}$ & 100 & 100 & oo \\
\hline 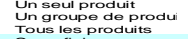 & $100^{\circ}$ & $100^{\circ}$ & 100 & 100 & $100^{\circ}$ & 100 & 100 & $100^{\circ}$ & $100^{\circ}-(x)$ & 100 & 100 & 100 & $100^{-}$ & $100^{\circ}$ & $100^{\circ}$ & $100^{\circ}$ & 100 & $100^{\circ}$ & $\stackrel{91}{9}$ & $\begin{array}{l}29 \\
71\end{array}$ & $\begin{array}{l}10 \\
90 \\
90\end{array}$ & \\
\hline 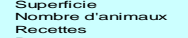 & & & & & & & & & & & & & & & & & & & & & & \\
\hline 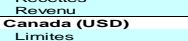 & $\frac{100}{1699}$ & $\frac{100}{1705}$ & $\frac{100}{600}$ & 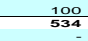 & $\begin{array}{l}100 \\
802\end{array}$ & $\frac{100}{2060}$ & $\begin{array}{l}100 \\
1309 \\
0\end{array}$ & $\begin{array}{l}100 \\
667\end{array}$ & $\begin{array}{l}\frac{100}{577} \\
7 ?-\end{array}$ & $\begin{array}{l}100 \\
668 \\
6-5\end{array}$ & $\begin{array}{c}\frac{100}{581} \\
581\end{array}$ & $\begin{array}{l}\frac{100}{582} \\
582\end{array}$ & $\begin{array}{l}100 \\
941 \\
941\end{array}$ & $\begin{array}{l}100 \\
1119\end{array}$ & 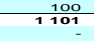 & $\frac{100}{211}$ & $\frac{100}{608}$ & $\frac{100}{183}$ & $\frac{100}{2046}$ & $\frac{100}{1549}$ & (1004 & \\
\hline 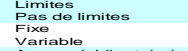 & $\begin{array}{r}100 \\
10 \\
99\end{array}$ & $\begin{array}{l}100^{\circ} \\
100\end{array}$ & $\begin{array}{l}100^{\circ} \\
100\end{array}$ & $\begin{array}{l}100^{\circ} \\
100\end{array}$ & $\begin{aligned} 100 \\
47 \\
53\end{aligned}$ & $\begin{array}{l}100 \\
100\end{array}$ & $\begin{array}{l}100 \\
100\end{array}$ & $\begin{array}{l}100 \\
100\end{array}$ & $\begin{array}{c}100 \\
39 \\
61 \\
61\end{array}$ & $\begin{array}{c}\text { roo } \\
\text { so } \\
50\end{array}$ & $\begin{array}{l}100 \\
42 \\
58\end{array}$ & 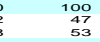 & 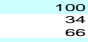 & 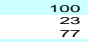 & 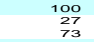 & 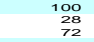 & 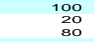 & $\begin{array}{l}100 \\
98 \\
98\end{array}$ & $\begin{array}{c}100 \\
98 \\
92\end{array}$ & $\begin{array}{l}100 \\
98 \\
98\end{array}$ & $\begin{array}{l}\text { loo } \\
\text { 10o } \\
100\end{array}$ & $\begin{array}{l}35 \\
35 \\
38 \\
38\end{array}$ \\
\hline 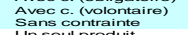 & 100 & 100 & & 100 & 100 & 100 & 100 & & & & & & & 100 & 100 & & & & & & & \\
\hline 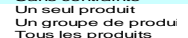 & $\begin{array}{l}56 \\
44\end{array}$ & $\begin{array}{l}60 \\
40 \\
40\end{array}$ & $\begin{array}{c}82 \\
18 \\
18\end{array}$ & 97 & $\begin{array}{l}40 \\
60 \\
60\end{array}$ & $\begin{array}{l}11 \\
89\end{array}$ & $\begin{array}{r}39 \\
61 \\
61\end{array}$ & $\begin{array}{l}52 \\
48\end{array}$ & $\begin{array}{l}44 \\
56 \\
56\end{array}$ & $\begin{aligned} 40 \\
53 \\
53\end{aligned}$ & 4 & $\begin{array}{l}44 \\
47 \\
4\end{array}$ & 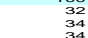 & $\begin{array}{l}39 \\
24 \\
33 \\
3\end{array}$ & $\begin{array}{l}52 \\
52 \\
27 \\
27\end{array}$ & $\begin{array}{l}52 \\
28 \\
20 \\
20\end{array}$ & $\begin{array}{l}59 \\
52 \\
21 \\
21\end{array}$ & $\begin{array}{l}24 \\
39 \\
37 \\
37\end{array}$ & $\begin{array}{l}45 \\
47 \\
38 \\
38\end{array}$ & $\begin{array}{l}34 \\
39 \\
47 \\
47\end{array}$ & $\begin{array}{c}26 \\
28 \\
{ }^{2}\end{array}$ & \\
\hline 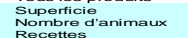 & $\begin{array}{c}57 \\
51 \\
41\end{array}$ & $\begin{aligned} 58 \\
2 \\
40\end{aligned}$ & $\begin{array}{c}91 \\
5 \\
4\end{array}$ & $\begin{aligned} 91 \\
6 \\
2 \\
2\end{aligned}$ & $\begin{array}{r}82 \\
15 \\
12\end{array}$ & $\begin{array}{r}39 \\
52 \\
59\end{array}$ & $\begin{array}{l}35 \\
64 \\
61\end{array}$ & 年 & $\begin{array}{l}37 \\
38 \\
56\end{array}$ & $\begin{array}{c}34 \\
57 \\
52\end{array}$ & $\begin{array}{l}41 \\
40 \\
402\end{array}$ & $\begin{array}{l}35 \\
31 \\
47 \\
47\end{array}$ & $\begin{array}{ll}26 \\
26 \\
34 & 1 \\
3\end{array}$ & $\begin{array}{r}35 \\
35 \\
23\end{array}$ & $\begin{array}{l}47 \\
47 \\
27\end{array}$ & $\begin{array}{l}49 \\
{ }^{4} \\
28 \\
28\end{array}$ & $\begin{array}{l}56 \\
53 \\
20\end{array}$ & $\begin{array}{l}21 \\
25 \\
25\end{array}$ & $\begin{array}{c}35 \\
11 \\
13 \\
3\end{array}$ & $\begin{aligned} 27 \\
2 \\
2\end{aligned}$ & $\begin{array}{c}6 \\
6 \\
0\end{array}$ & \\
\hline 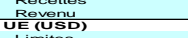 & 2611 & 3753 & 4094 & $\frac{2}{109}$ & 972 & 852 & $\frac{61}{659}$ & 178 & (253 & $\begin{array}{c}\frac{57}{510} \\
5105\end{array}$ & $\frac{47}{259}$ & $\begin{array}{l}78 \\
639 \\
639\end{array}$ & $\begin{array}{l}34 \\
037 \\
057\end{array}$ & $\begin{array}{l}\frac{23}{327} \\
429 \\
\end{array}$ & $\begin{array}{l}27 \\
5493 \\
493\end{array}$ & $\begin{array}{l}\frac{20}{222} \\
222 \\
\end{array}$ & $\begin{array}{ll}201 \\
693 \\
693\end{array}$ & $\begin{array}{l}250 \\
512 \\
512 \\
5\end{array}$ & $\frac{53}{216}$ & $\begin{array}{l}63 \\
\frac{63}{103} \\
103\end{array}$ & & \\
\hline $\begin{array}{l}\text { Limites limites } \\
\text { Fas die dimites }\end{array}$ & $\begin{array}{l}95 \\
95 \\
65\end{array}$ & $\begin{array}{l}8 \\
92 \\
60\end{array}$ & $\begin{array}{l}73 \\
93 \\
59\end{array}$ & $\begin{array}{l}11 \\
6 \mathbf{6} \\
6 \mathbf{6}\end{array}$ & $\begin{array}{l}10 \\
\text { so } \\
68\end{array}$ & $\begin{array}{l}13 \\
77 \\
77\end{array}$ & $\begin{array}{l}69 \\
31 \\
31\end{array}$ & $\begin{array}{l}83 \\
160 \\
90\end{array}$ & $\begin{array}{l}82 \\
18 \\
92 \\
92\end{array}$ & $\begin{array}{l}75 \\
75 \\
94\end{array}$ & $\begin{array}{l}73 \\
92 \\
95\end{array}$ & $\begin{array}{l}74 \\
765 \\
95\end{array}$ & $\begin{array}{l}75 \\
95 \\
94\end{array}$ & $\begin{array}{c}75 \\
75 \\
94\end{array}$ & $\begin{array}{l}82 \\
18 \\
95 \\
95\end{array}$ & $\begin{array}{l}81 \\
19 \\
96\end{array}$ & $\begin{array}{l}83 \\
17 \\
93\end{array}$ & $\begin{array}{l}82 \\
\text { sid } \\
94\end{array}$ & $\begin{array}{l}84 \\
164 \\
94\end{array}$ & $\begin{array}{l}73 \\
27 \\
95 \\
95\end{array}$ & $\begin{array}{l}59 \\
44 \\
944\end{array}$ & $\begin{array}{c}40 \\
93 \\
93\end{array}$ \\
\hline 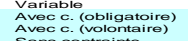 & $\begin{array}{l}35 \\
35 \\
35\end{array}$ & $\begin{array}{l}40 \\
26 \\
26 \\
\end{array}$ & $\begin{array}{l}41 \\
23 \\
23\end{array}$ & $\begin{array}{l}34 \\
27 \\
27 \\
2\end{array}$ & $\begin{array}{l}32 \\
32 \\
32\end{array}$ & $\begin{array}{l}29 \\
32 \\
32\end{array}$ & $\begin{array}{l}19 \\
34 \\
34\end{array}$ & $\begin{array}{l}10 \\
28 \\
28 \\
2\end{array}$ & $\begin{array}{c}88 \\
18 \\
28 \\
\end{array}$ & 30 & $\begin{array}{r}5 \\
5 \\
32 \\
32\end{array}$ & $\begin{array}{r}5 \\
35 \\
35\end{array}$ & $\begin{array}{r}6 \\
5 \\
35\end{array}$ & $\underset{36}{\frac{6}{7}}$ & $\begin{array}{r}\frac{5}{3} \\
3^{7}\end{array}$ & $\begin{array}{r}4 \\
37 \\
37\end{array}$ & $\begin{array}{r}7 \\
36 \\
36\end{array}$ & $\begin{aligned} 5 \\
30^{3}\end{aligned}$ & $\begin{array}{l}46 \\
46 \\
37\end{array}$ & $\begin{array}{l}35 \\
39 \\
49\end{array}$ & $\begin{array}{c}18 \\
18 \\
66\end{array}$ & $\begin{array}{r}7 \\
62 \\
62\end{array}$ \\
\hline 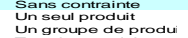 & $\begin{array}{c}65 \\
58 \\
33\end{array}$ & $\begin{array}{l}74 \\
67 \\
26 \\
26\end{array}$ & $\begin{array}{l}77 \\
69 \\
23 \\
23\end{array}$ & $\begin{array}{l}72 \\
65 \\
27\end{array}$ & $\begin{array}{l}88 \\
57 \\
25\end{array}$ & $\begin{array}{l}67 \\
69 \\
29\end{array}$ & 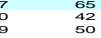 & $\begin{array}{l}64 \\
32 \\
62 \\
62\end{array}$ & $\begin{array}{l}62 \\
\text { 30 } \\
64\end{array}$ & $\begin{array}{l}63 \\
27 \\
63\end{array}$ & $\begin{array}{l}62 \\
28 \\
62 \\
62\end{array}$ & 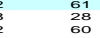 & $\begin{array}{c}61 \\
29 \\
60 \\
60\end{array}$ & $\begin{array}{l}57 \\
\text { 30 } \\
58\end{array}$ & $\begin{array}{l}54 \\
\text { 39 } \\
49\end{array}$ & 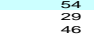 & $\begin{array}{l}56 \\
37 \\
43\end{array}$ & $\begin{array}{l}55 \\
33 \\
45\end{array}$ & $\begin{array}{l}37 \\
32 \\
47\end{array}$ & $\begin{array}{l}15 \\
24 \\
41\end{array}$ & $\begin{array}{l}17 \\
27 \\
27\end{array}$ & \\
\hline 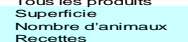 & $\begin{array}{l}16 \\
79 \\
79\end{array}$ & $\begin{array}{l}15 \\
82 \\
82 \\
1\end{array}$ & $\begin{array}{l}18 \\
78 \\
78\end{array}$ & $\begin{array}{l}18 \\
79 \\
79\end{array}$ & $\begin{array}{l}18 \\
28 \\
67\end{array}$ & $\begin{array}{l}14 \\
23 \\
73\end{array}$ & $\begin{array}{l}52 \\
47 \\
47\end{array}$ & 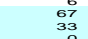 & $\begin{array}{l}66 \\
33 \\
33 \\
\end{array}$ & $\begin{array}{l}16 \\
61 \\
31\end{array}$ & $\begin{aligned} 109 \\
31 \\
31\end{aligned}$ & 势 & 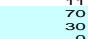 & 年 & 年 78 & $\begin{array}{ll}24 \\
30 \\
20\end{array}$ & $\begin{array}{l}21 \\
72 \\
28\end{array}$ & $\begin{array}{l}27 \\
73 \\
26 \\
26\end{array}$ & $\begin{array}{l}21 \\
72 \\
27\end{array}$ & $\begin{array}{l}38 \\
72 \\
20\end{array}$ & $\begin{array}{ll}52 \\
81 \\
16\end{array}$ & $\begin{array}{l}50 \\
79 \\
17 \\
3\end{array}$ \\
\hline 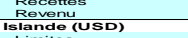 & & & & & & & & & & & & & & & & & & & & & & \\
\hline 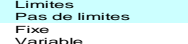 & & & & & & & & & & & & & & & & & & & & & & \\
\hline 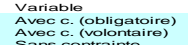 & & & & & & & & & & & & & & & & & & & & & & \\
\hline 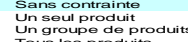 & & & & & & & & & & & & & & & & & & & & & 。o & \\
\hline 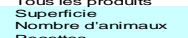 & & & & & & & & & & & & & & & & & & & & & $100^{\circ}$ & 100 \\
\hline 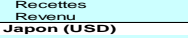 & & & & & & & & & & & & & & & & & & & 76 & 268 & 204 & \\
\hline $\begin{array}{l}\text { Limites limites } \\
\text { Fas de limite } \\
\text { File }\end{array}$ & & & & & & & & & & & & & & & & & & & $\begin{array}{l}100 \\
100\end{array}$ & $\begin{array}{l}39 \\
61 \\
61\end{array}$ & 67 & \\
\hline 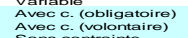 & & & & & & & & & & & & & & & & & & & & & & \\
\hline 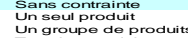 & & & & & & & & & & & & & & & & & & & $\begin{array}{c}100 \\
49 \\
51\end{array}$ & 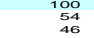 & 52 & \\
\hline 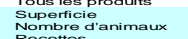 & & & & & & & & & & & & & & & & & & & $100^{\circ}$ & $=i$ & & \\
\hline 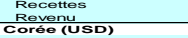 & 17 & 51 & 37 & 31 & 356 & 375 & 403 & 327 & 373 & 326 & 284 & 203 & & 173 & 337 & 53 & 65 & 039 & 906 & $\begin{array}{l}39 \\
1512\end{array}$ & $\frac{33}{084}$ & 816 \\
\hline $\begin{array}{l}\text { Limites limites } \\
\text { Pas de dimites }\end{array}$ & 10o & $\begin{array}{l}100 \\
35\end{array}$ & 1000 & 100 & $\begin{aligned} 100 \\
97\end{aligned}$ & $\begin{array}{c}100 \\
98 \\
98\end{array}$ & 100 & 100 & 100 & ${ }_{81}^{100}$ & $\begin{array}{l}100 \\
\text { 9o } \\
\text { 9o }\end{array}$ & $\begin{array}{l}100 \\
87\end{array}$ & $\begin{array}{l}92 \\
98 \\
67\end{array}$ & 100 & $\begin{array}{c}100 \\
82\end{array}$ & 100 & 100 & $\begin{array}{l}98 \\
92 \\
92\end{array}$ & $\begin{array}{l}93 \\
96 \\
96\end{array}$ & $\begin{array}{l}95 \\
98 \\
98 \\
\end{array}$ & $100^{\circ}$ & \\
\hline 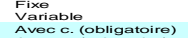 & & & & & & & & & & & & & & 53 & & & & $\begin{array}{ll}88 \\
39\end{array}$ & ${ }^{6}$ & & & \\
\hline 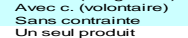 & $100^{\circ}$ & 100 & 100 & 100 & $\begin{array}{r}100 \\
0\end{array}$ & $100^{\circ}$ & 100 & 100 & 100 & $100^{-}$ & 100 & $100^{\circ}$ & 100 & 97 & 98 & 70 & 54 & $\begin{array}{l}61 \\
2\end{array}$ & $5 \overline{3}$ & $\begin{array}{l}99 \\
58\end{array}$ & $\begin{array}{l}99 \\
45\end{array}$ & \\
\hline 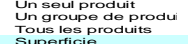 & $100^{\circ}$ & $100^{\circ}$ & 100 & 100 & $\begin{array}{r}0 \\
100\end{array}$ & $100^{\circ}$ & 100 & $99^{2}$ & $97^{\frac{3}{7}}$ & $\begin{array}{r}55 \\
95\end{array}$ & $\begin{array}{r}4 \\
96\end{array}$ & 95 & 92 & $\begin{array}{l}7 \\
93 \\
93\end{array}$ & $\begin{array}{c}0 \overline{1} \\
94 \\
2\end{array}$ & $\begin{array}{l}38 \\
62 \\
62\end{array}$ & $\begin{array}{c}53 \\
47 \\
46 \\
4\end{array}$ & $\begin{array}{l}50 \\
499 \\
45\end{array}$ & $\begin{array}{l}55 \\
54 \\
54\end{array}$ & $\begin{array}{l}06 \\
36 \\
63\end{array}$ & $\begin{array}{l}3 \\
53 \\
46\end{array}$ & \\
\hline 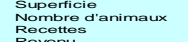 & 10 & 65 & 66 & 43 & $\stackrel{0}{3}$ & 2 & 4 & ${ }_{6}^{2}$ & $\begin{array}{l}33 \\
28 \\
28\end{array}$ & $\begin{array}{r}5 \\
19 \\
19\end{array}$ & $\begin{aligned} 4 \\
10 \\
86\end{aligned}$ & $\begin{array}{c}5 \\
13 \\
82 \\
82\end{array}$ & 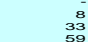 & 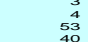 & 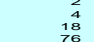 & $\begin{array}{r}8 \\
59 \\
5\end{array}$ & $\begin{array}{l}14 \\
33\end{array}$ & 量 & $\begin{array}{r}4 \\
4 \\
4\end{array}$ & $\begin{array}{r}3 \\
3 \\
3 \\
3\end{array}$ & & \\
\hline
\end{tabular}

ANALYSE DE LA COMPOSITION DU SOUTIEN AUX PRODUCTEURS : NOUVEAUX OUTILS ET MÉTHODES ๑ OECD 2010 
Tableau annexe 6. Catégorie C (PC) : composition de la catégorie par pays et étiquette, 1986-2007 (suite.)

\begin{tabular}{|c|c|c|c|c|c|c|c|c|c|c|c|c|c|c|c|c|c|c|c|c|c|c|}
\hline Mexique (USD) & 1986 & 1987 & $\begin{array}{r}1988 \\
11\end{array}$ & 1989 & 1990 & $\begin{array}{l}1991 \\
3.3\end{array}$ & 1992 & 1993 & $\begin{aligned} 1994 \\
-180\end{aligned}$ & $\begin{array}{l}1995 \\
12 \\
12\end{array}$ & $\begin{array}{l}1996 \\
21 \\
21\end{array}$ & $\begin{array}{l}1997 \\
59 \\
\end{array}$ & $\begin{array}{c}1998 \\
103 \\
607\end{array}$ & $\begin{array}{l}1999 \\
91\end{array}$ & $\begin{array}{l}2000 \\
1000\end{array}$ & $\begin{array}{l}2001 \\
124 \\
24\end{array}$ & $\begin{array}{l}2002 \\
125 \\
125\end{array}$ & $\begin{array}{l}2003 \\
128 \\
\frac{20}{25}\end{array}$ & 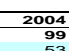 & $\begin{array}{l}2005 \\
68\end{array}$ & $\begin{array}{l}2006 \\
139 \\
390\end{array}$ & $\frac{2007}{247}$ \\
\hline $\begin{array}{l}\text { Limitses } \\
\text { Pam dimites } \\
\text { Fixd }\end{array}$ & & & 100 & & & & & & & & $\begin{array}{l}100 \\
100\end{array}$ & $\begin{array}{r}36 \\
64 \\
100\end{array}$ & $\begin{array}{l}57 \\
43 \\
78\end{array}$ & $\begin{array}{r}31 \\
69 \\
100\end{array}$ & $\begin{array}{r}42 \\
58 \\
100\end{array}$ & $\begin{array}{l}49 \\
51 \\
74\end{array}$ & $\begin{array}{l}55 \\
45 \\
69\end{array}$ & $\begin{array}{l}45 \\
55 \\
15\end{array}$ & $\begin{array}{l}53 \\
47\end{array}$ & $\begin{array}{l}35 \\
65\end{array}$ & $\begin{array}{l}70 \\
30\end{array}$ & $\begin{array}{l}84 \\
16\end{array}$ \\
\hline $\begin{array}{l}\text { Variable } \\
\text { Avece c. (obligatoire) }\end{array}$ & & & 100 & 100 & 100 & 100 & 100 & 100 & 100 & 100 & & & & & & 26 & & 85 & 100 & 100 & 100 & 100 \\
\hline $\begin{array}{l}\text { Avec c. (. (volontaire) } \\
\text { Sans contrainte }\end{array}$ & & & $100^{\circ}-(x)$ & 100 & $100^{\circ}$ & 100 & $100^{\circ}$ & 100 & 100 & $100^{\circ}$ & 100 & $100^{\circ}-(-) \cdot(x)$ & 100 & 100 & 100 & 100 & 100 & 100 & 100 & 100 & 100 & 100 \\
\hline 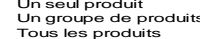 & & & $100^{\circ}-(x-3)$ & 100 & $100^{\circ}-(x)-10$ & 100 & $100^{\circ}-(x)-10$ & $100^{-}-$ & 100 & $100^{\circ}-$ & $\begin{array}{c}99 \\
1\end{array}$ & $\begin{array}{r}33 \\
2 \\
64\end{array}$ & $\begin{array}{l}33 \\
25 \\
43\end{array}$ & $\begin{array}{r}28 \\
3 \\
69 \\
69\end{array}$ & $\begin{array}{l}39 \\
3 \\
58\end{array}$ & $\begin{array}{l}23 \\
26 \\
51\end{array}$ & $\begin{array}{l}13 \\
43 \\
45\end{array}$ & $\begin{array}{l}45 \\
55\end{array}$ & $\begin{array}{l}53 \\
47\end{array}$ & $\begin{array}{l}35 \\
65 \\
65\end{array}$ & $\begin{array}{l}70 \\
30\end{array}$ & $\begin{array}{l}84 \\
16\end{array}$ \\
\hline $\begin{array}{l}\text { Superficie } \\
\text { Nombre d'animaux } \\
\text { Recettes }\end{array}$ & & & 100 & 100 & 100 & 100 & $100^{\circ}$ & $100^{\circ}$ & 100 & 100 & 100 & 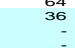 & $\begin{array}{l}43 \\
57\end{array}$ & $\begin{array}{l}69 \\
31\end{array}$ & $\begin{array}{l}58 \\
42\end{array}$ & $\begin{array}{l}51 \\
49\end{array}$ & $\begin{array}{l}45 \\
55\end{array}$ & $\begin{array}{l}55 \\
85\end{array}$ & $\begin{array}{l}47 \\
100\end{array}$ & $\begin{array}{l}65 \\
100\end{array}$ & $\begin{array}{l}300 \\
100\end{array}$ & $\begin{array}{r}16 \\
100\end{array}$ \\
\hline $\begin{array}{l}\text { Revenu } \\
\text { Nelle-zélande (USD) }\end{array}$ & 15 & 11 & 51 & 18 & 17 & 10 & 3 & & $\mathrm{o}$ & & $i$ & $\frac{64}{0}$ & $\frac{43}{0}$ & $\begin{array}{r}69 \\
0\end{array}$ & $\frac{58}{2}$ & $\frac{51}{0}$ & $\frac{45}{-}$ & $\frac{15}{0}$ & 4 & 30 & 5 & $\div$ \\
\hline $\begin{array}{l}\text { Limites ilmites } \\
\text { Pas de limites }\end{array}$ & 100 & 100 & 100 & 100 & $100^{\circ}$ & 100 & $100^{\circ}$ & 100 & 100 & & 100 & $100^{\circ}$ & 100 & 100 & $100^{\circ}$ & $100^{\circ}-(x)-3$ & & $100^{\circ}$ & 100 & 100 & 100 & $100^{\circ}$ \\
\hline $\begin{array}{l}\text { Five } \\
\text { Nariable } \\
\text { Avece cobigatire) }\end{array}$ & $\begin{array}{l}78 \\
22\end{array}$ & & $\begin{array}{l}18 \\
82\end{array}$ & $\begin{array}{l}39 \\
61\end{array}$ & $\begin{array}{l}12 \\
88\end{array}$ & 100 & $100^{\circ}$ & $100^{-}$ & 100 & & $100^{\circ}-(x)$ & $100^{\circ}$ & 100 & 100 & $100^{\circ}$ & 100 & & $100^{\circ}$ & $100^{\circ}$ & 100 & $100^{-}$ & $100^{\circ}$ \\
\hline 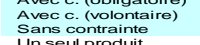 & $100^{\circ}-(x)$ & $100^{\circ}$ & 100 & 100 & $100^{\circ}$ & 100 & $100^{\circ}$ & $100^{\circ}$ & 100 & & $100^{\circ}-(x)$ & $100^{\circ}$ & $100^{\circ}$ & 100 & $100^{\circ}$ & $100^{-}$ & & $100^{\circ}$ & 100 & $100^{\circ}$ & 100 & $100^{\circ}$ \\
\hline 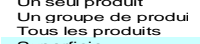 & $100^{\circ}$ & $100^{\circ}$ & $100^{\circ}-(x)$ & $100^{\circ}-(x)$ & $100^{\circ}$ & 100 & $100^{\circ}$ & $100^{\circ}-(x)$ & 100 & & $100^{\circ}-(x)$ & $100^{\circ}$ & $100^{\circ}-(-3)$ & 100 & $100^{\circ}$ & 100 & & 100 & $100^{-}$ & $100^{-}$ & $100^{-}$ & $100^{\circ}$ \\
\hline $\begin{array}{l}\text { Superdicieleanimaux } \\
\text { Nomberedies } \\
\text { Recetes }\end{array}$ & & & & & & & & & & & & & & & & & & & & & & \\
\hline 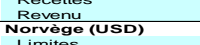 & $\begin{array}{l}100 \\
429\end{array}$ & $\begin{array}{l}100 \\
535 \\
35\end{array}$ & $\frac{100}{607}$ & $\begin{array}{l}100 \\
584\end{array}$ & $\begin{array}{l}100 \\
700 \\
702\end{array}$ & $\frac{100}{852}$ & $\begin{aligned} 100 \\
1018 \\
1018\end{aligned}$ & $\frac{100}{936}$ & $\begin{array}{l}100 \\
930 \\
932\end{array}$ & 988 & $\begin{array}{l}100 \\
938 \\
938\end{array}-3$ & $\begin{array}{l}100 \\
912 \\
912\end{array}$ & $\begin{array}{l}100 \\
921 \\
925\end{array}$ & $\begin{array}{l}\frac{100}{887} \\
887\end{array}$ & $\begin{array}{l}100 \\
836\end{array}$ & $\frac{100}{808}$ & 948 & $\frac{100}{789}$ & $\frac{100}{830}$ & $\frac{100}{844}$ & $\frac{100}{886}$ & $\begin{array}{l}100 \\
997 \\
997\end{array}$ \\
\hline $\begin{array}{l}\text { Pas de limites } \\
\text { Fire diable } \\
\text { variable }\end{array}$ & $\begin{array}{r}44 \\
56 \\
100\end{array}$ & $\begin{array}{r}48 \\
580 \\
100\end{array}$ & $\begin{array}{r}53 \\
100\end{array}$ & $\begin{aligned} 54 \\
100 \\
100\end{aligned}$ & $\begin{array}{r}57 \\
5100 \\
100\end{array}$ & $\begin{array}{r}62 \\
62 \\
100\end{array}$ & $\begin{aligned} 65 \\
65 \\
100\end{aligned}$ & $\begin{array}{r}64 \\
66 \\
100\end{array}$ & $\begin{array}{r}67 \\
67 \\
100\end{array}$ & $\begin{array}{r}30 \\
700 \\
100\end{array}$ & $\begin{array}{r}74 \\
100 \\
100\end{array}$ & $\begin{array}{r}76 \\
100 \\
100\end{array}$ & $\begin{array}{r}75 \\
100 \\
100\end{array}$ & $\begin{aligned} 69 \\
60 \\
100\end{aligned}$ & $\begin{array}{r}69 \\
100\end{array}$ & $\begin{array}{r}69 \\
100\end{array}$ & $\begin{array}{r}30 \\
700 \\
100\end{array}$ & $\begin{array}{r}60 \\
600 \\
100\end{array}$ & $\begin{array}{r}59 \\
500 \\
100\end{array}$ & $\begin{array}{r}50 \\
61 \\
100\end{array}$ & $\begin{array}{r}63 \\
630\end{array}$ & $\begin{array}{r}68 \\
68 \\
100\end{array}$ \\
\hline $\begin{array}{l}\text { Avec c. (. (Obligatoire) } \\
\text { Avec c. (volare) } \\
\text { Sans contrainte }\end{array}$ & 100 & $100^{\circ}$ & 100 & 100 & 100 & 100 & 100 & 100 & 99 & $98^{2}$ & 98 & 98 & 98 & $98^{2}-1-30$ & 98 & 100 & $\begin{array}{l}3 \\
97\end{array}$ & 96 & 96 & 96 & 93 & $\begin{array}{r}8 \\
92\end{array}$ \\
\hline 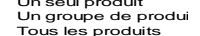 & 99 & $\begin{array}{r}98 \\
2\end{array}$ & 99 & 99 & 99 & 99 & 97 & $\begin{array}{r}96 \\
4\end{array}$ & 96 & $\begin{array}{r}96 \\
4\end{array}$ & 97 & 97 & 98 & 97 & 93 & $\begin{array}{r}92 \\
8\end{array}$ & $\begin{array}{c}90 \\
10 \\
10\end{array}$ & $\begin{array}{l}85 \\
15 \\
15\end{array}$ & $\begin{array}{l}87 \\
13\end{array}$ & 86 & 83 & $7 \overline{7}$ \\
\hline $\begin{array}{l}\text { Superficie } \\
\text { Nombre d'animaux } \\
\text { Recettes }\end{array}$ & $\begin{array}{l}25 \\
75\end{array}$ & $\begin{array}{l}29 \\
71\end{array}$ & $\begin{array}{l}27 \\
73 \\
\end{array}$ & $\begin{array}{l}27 \\
73 \\
\end{array}$ & $\begin{array}{l}29 \\
71\end{array}$ & $\begin{array}{l}38 \\
62 \\
62\end{array}$ & $\begin{array}{l}44 \\
56\end{array}$ & $\begin{array}{l}46 \\
54 \\
54\end{array}$ & $\begin{array}{c}46 \\
54 \\
54\end{array}-100$ & $\begin{array}{l}48 \\
52 \\
52\end{array}$ & $\begin{array}{l}56 \\
44\end{array}$ & $\begin{array}{l}56 \\
44\end{array}$ & $\begin{array}{l}58 \\
42\end{array}$ & $\begin{array}{c}51 \\
48\end{array}$ & $\begin{array}{l}48 \\
48\end{array}$ & $\begin{array}{l}46 \\
47 \\
47\end{array}$ & $\begin{array}{l}10 \\
46 \\
48\end{array}$ & $\begin{array}{l}15 \\
31 \\
58\end{array}$ & $\begin{array}{l}13 \\
30 \\
60\end{array}$ & $\begin{array}{l}14 \\
32 \\
58\end{array}$ & $\begin{array}{l}17 \\
35 \\
56\end{array}$ & $\begin{array}{l}23 \\
37 \\
49\end{array}$ \\
\hline $\begin{array}{l}\text { Revenu } \\
\text { Suisse (USD) }\end{array}$ & 310 & 399 & 467 & 504 & 553 & 730 & 741 & 547 & 587 & 770 & 1053 & 963 & 908 & 590 & $\frac{4}{545}$ & $\frac{7}{522}$ & $\frac{7}{615}$ & $\frac{11}{726}$ & $\begin{array}{r}10 \\
791\end{array}$ & $\frac{10}{796}$ & $\begin{array}{r}10 \\
796\end{array}$ & $\frac{14}{926}$ \\
\hline 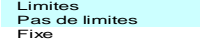 & $\begin{array}{r}25 \\
75 \\
100 \\
100\end{array}$ & $\begin{array}{r}24 \\
76 \\
100 \\
100\end{array}$ & $\begin{array}{r}31 \\
69 \\
100\end{array}$ & $\begin{array}{r}38 \\
62 \\
100\end{array}$ & $\begin{array}{r}35 \\
65 \\
100 \\
100\end{array}$ & $\begin{aligned} 40 \\
60 \\
100\end{aligned}$ & $\begin{aligned} 40 \\
60 \\
100\end{aligned}$ & $\begin{array}{r}98 \\
100^{8} \\
100\end{array}$ & $\begin{array}{r}93^{7} \\
100\end{array}$ & $\begin{aligned} 96 \\
100 \\
100\end{aligned}$ & $\begin{array}{r}94 \\
96 \\
100\end{array}$ & $\begin{aligned} 96 \\
100\end{aligned}$ & $\begin{array}{r}94 \\
96 \\
100 \\
100\end{array}$ & $\begin{array}{r}11 \\
89 \\
100\end{array}$ & $\begin{aligned} 100 \\
\text { 9o } \\
100\end{aligned}$ & $\begin{array}{c}100 \\
100\end{array}$ & $\begin{array}{l}100 \\
100\end{array}$ & $\begin{array}{l}100 \\
100 \\
100\end{array}$ & $\begin{array}{l}100 \\
100 \\
100\end{array}$ & $\begin{array}{l}100 \\
100\end{array}$ & $\begin{array}{l}100 \\
100 \\
100\end{array}$ & $\begin{array}{l}100 \\
100\end{array}$ \\
\hline $\begin{array}{l}\text { Variable (obligatoire) } \\
\text { Avec c. (volontaire) }\end{array}$ & $\begin{array}{l}45 \\
12\end{array}$ & $\begin{array}{l}48 \\
10\end{array}$ & $\begin{array}{r}45 \\
8\end{array}$ & $\begin{array}{l}41 \\
7\end{array}$ & ${ }_{8}^{44}$ & $\begin{array}{r}35 \\
6 \\
6\end{array}$ & $\begin{array}{l}37 \\
11\end{array}$ & $\begin{array}{r}59 \\
19 \\
\end{array}$ & $\begin{array}{l}57 \\
22\end{array}$ & $\begin{array}{l}54 \\
28\end{array}$ & $\begin{array}{l}45 \\
44\end{array}$ & $\begin{array}{l}40 \\
50\end{array}$ & $\begin{array}{l}38 \\
51\end{array}$ & $\begin{array}{l}68 \\
19 \\
19\end{array}-3$ & $\begin{array}{l}69 \\
20\end{array}$ & $\begin{array}{l}73 \\
26 \\
26\end{array} \mathrm{l}$ & $\begin{array}{l}74 \\
25\end{array}$ & $\begin{array}{l}73 \\
26 \\
\end{array}$ & $\begin{array}{l}72 \\
27 \\
27\end{array} 10$ & $\begin{array}{l}72 \\
27 \\
27\end{array} \mathrm{l}$ & $\begin{array}{l}71 \\
28\end{array}$ & $\begin{array}{l}74 \\
25\end{array}$ \\
\hline $\begin{array}{l}\text { Sans contrainte } \\
\text { Un seul produit } \\
\text { Un groupe de produi }\end{array}$ & $\begin{array}{l}43 \\
30 \\
70 \\
70\end{array}$ & $\begin{array}{l}28 \\
72 \\
72\end{array}$ & $\begin{array}{l}48 \\
24 \\
76\end{array}$ & $\begin{array}{l}53 \\
21 \\
79\end{array}$ & $\begin{array}{l}48 \\
21 \\
79\end{array}$ & $\begin{array}{l}59 \\
24 \\
76\end{array}$ & $\begin{array}{l}52 \\
18 \\
82\end{array}$ & $\begin{array}{l}23 \\
22 \\
78\end{array}$ & $\begin{array}{l}20 \\
18 \\
82\end{array}$ & $\begin{array}{l}18 \\
15 \\
85\end{array}$ & $\begin{array}{l}12 \\
99 \\
91 \\
91\end{array}$ & $\begin{array}{l}10 \\
99 \\
91\end{array}$ & $\begin{array}{l}11 \\
9 \\
91\end{array}$ & $\begin{array}{r}12 \\
1 \\
99\end{array}$ & $\begin{array}{l}11 \\
1 \\
99\end{array}$ & 99 & $\begin{array}{c}1 \\
1 \\
99\end{array}$ & 99 & 99 & $\begin{array}{ccc}1 & 1 \\
9 & 9 & 1\end{array}$ & $\begin{array}{l}1 \\
99 \\
1\end{array}$ & $\begin{array}{c}1 \\
1 \\
99\end{array}$ \\
\hline $\begin{array}{l}\text { Superficiedientima } \\
\text { Nombre dianimaux }\end{array}$ & $\begin{array}{l}44 \\
56\end{array}$ & $\begin{array}{l}42 \\
58\end{array}$ & $\begin{array}{l}42 \\
51\end{array}$ & $\begin{array}{l}36 \\
47 \\
47\end{array}-10$ & $\begin{array}{l}36 \\
52 \\
52\end{array}-10$ & $\begin{array}{l}26 \\
50\end{array}$ & $\begin{array}{l}25 \\
43\end{array}$ & $\begin{array}{l}48 \\
52\end{array}$ & $\begin{array}{l}51 \\
49\end{array}-10$ & $\begin{array}{l}58 \\
42\end{array}$ & $\begin{array}{l}69 \\
31\end{array}$ & $\begin{array}{l}71 \\
29\end{array}$ & $\begin{array}{l}69 \\
31\end{array}$ & $\begin{array}{l}32 \\
68 \\
68\end{array}$ & $\begin{array}{l}33 \\
67 \\
67\end{array}$ & $\begin{array}{l}24 \\
76\end{array}$ & $\begin{array}{l}22 \\
78\end{array}$ & $\begin{array}{l}22 \\
78 \\
\end{array}$ & $\begin{array}{l}23 \\
77 \\
-10\end{array}$ & $\begin{array}{l}22 \\
78 \\
78\end{array} \mathrm{l}$ & $\begin{array}{l}22 \\
78 \\
78\end{array}-10$ & $\begin{array}{l}20 \\
80\end{array}$ \\
\hline $\begin{array}{l}\text { Revenu } \\
\text { Turquie (USD) } \\
\text { Limites }\end{array}$ & & & 7 & 16 & 12 & 24 & 32 & & $\begin{array}{l}135 \\
100\end{array}$ & $\begin{array}{l}64 \\
100\end{array}$ & $\begin{array}{c}76 \\
100\end{array}$ & $\begin{array}{c}16 \\
100\end{array}$ & $\begin{array}{r}14 \\
100\end{array}$ & $100^{7}$ & $\begin{array}{r}25 \\
100 \\
\end{array}$ & $\begin{array}{c}21 \\
100\end{array}$ & $\begin{array}{r}28 \\
100\end{array}$ & $\begin{array}{l}26 \\
100\end{array}$ & 100 & $\begin{array}{l}35 \\
100\end{array}$ & $\begin{array}{r}49 \\
100\end{array}$ & $\begin{array}{c}66 \\
100\end{array}$ \\
\hline $\begin{array}{l}\text { Pas de limites } \\
\text { Fare } \\
\text { Variable }\end{array}$ & & & & & & & & & 100 & $100^{\circ}$ & 100 & $100^{\circ}$ & $100^{\circ}$ & 100 & $100^{\circ}$ & 100 & $99^{-3}$ & 91 & 97 & $10 \mathrm{o}$ & $98^{2}-10$ & $\begin{array}{l}1 \\
99 \\
9\end{array}$ \\
\hline 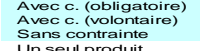 & & & & & & & & & $\begin{array}{l}100 \\
100\end{array}$ & $\begin{array}{l}100 \\
100\end{array}$ & 100 & $\begin{array}{l}100 \\
100\end{array}$ & $\begin{array}{l}100 \\
100\end{array}$ & $\begin{array}{l}100 \\
100\end{array}$ & $\begin{array}{l}100 \\
100\end{array}$ & $\begin{array}{l}100 \\
100\end{array}$ & $\begin{array}{ll}100 \\
97\end{array}$ & 100 & 100 & $\begin{array}{l}100 \\
100\end{array}$ & $\begin{array}{l}28 \\
98 \\
95\end{array}-10$ & 99 \\
\hline 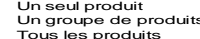 & & & & & & & & & 100 & & & & & & & & $\begin{array}{l}97 \\
3\end{array}$ & & 97 & 100 & 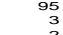 & $\begin{array}{l}68 \\
{ }_{32}\end{array}$ \\
\hline 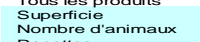 & & & & & & & & & 100 & 100 & 100 & 100 & 100 & 100 & 100 & 100 & 97 & 99 & 97 & 100 & 97 & 68 \\
\hline $\begin{array}{c}\text { Fevenu } \\
\text { Etats-unis (USD) }\end{array}$ & 13474 & 12883 & 10344 & 7890 & 7883 & 6952 & 7836 & 8426 & 6298 & 3024 & 1283 & 1169 & 4097 & 4404 & 5175 & 5106 & 6684 & 5080 & 4088 & $386 \overline{8}$ & $\frac{3}{3240}$ & $\frac{32}{1802}$ \\
\hline $\begin{array}{l}\text { Limites ilmites } \\
\text { Pas de limites }\end{array}$ & 83 & & & $\begin{array}{l}63 \\
37 \\
37\end{array}$ & $\begin{array}{l}81 \\
19 \\
19\end{array}-1$ & $\begin{array}{l}73 \\
27 \\
27\end{array}$ & $\begin{array}{l}78 \\
22\end{array}$ & $\begin{array}{l}51 \\
49 \\
49\end{array}-10$ & $\begin{array}{l}84 \\
16 \\
18\end{array}$ & $\begin{array}{l}50 \\
50\end{array}$ & $100^{\circ}-(x)$ & & 100 & 100 & $100^{\circ}-(x)$ & $100^{-}$ & & & $100^{-}$ & & $100^{\circ}$ & 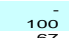 \\
\hline 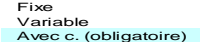 & $\begin{array}{c}17 \\
83 \\
9\end{array}$ & $\begin{array}{c}18 \\
82 \\
13\end{array}$ & $\begin{array}{l}44 \\
56 \\
46\end{array}$ & $\begin{array}{l}29 \\
71 \\
27\end{array}$ & $\begin{array}{l}14 \\
86 \\
10\end{array}$ & $\begin{array}{l}21 \\
79 \\
15\end{array}$ & $\begin{array}{l}18 \\
82 \\
14\end{array}$ & $\begin{array}{l}36 \\
64 \\
43\end{array}$ & $\begin{aligned} 18 \\
82 \\
8\end{aligned}$ & $\begin{array}{l}19 \\
81 \\
30\end{array}$ & $\begin{array}{l}50 \\
50 \\
50\end{array}$ & $\begin{array}{l}90 \\
10 \\
10\end{array}$ & $\begin{array}{l}82 \\
18 \\
65\end{array}$ & $\begin{array}{l}65 \\
35 \\
62\end{array}$ & $\begin{array}{l}73 \\
27 \\
62\end{array}$ & $\begin{array}{r}65 \\
35 \\
50\end{array}$ & $\begin{array}{l}55 \\
45 \\
68\end{array}$ & $\begin{array}{l}67 \\
33 \\
49\end{array}$ & $\begin{array}{l}72 \\
28 \\
48\end{array}$ & $\begin{array}{l}77 \\
23 \\
55\end{array}$ & $\begin{array}{r}50 \\
50 \\
50\end{array}$ & $\begin{array}{c}67 \\
33 \\
33\end{array}$ \\
\hline 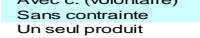 & $\begin{array}{l}91 \\
85\end{array}$ & $\begin{array}{l}87 \\
94\end{array}$ & $\begin{array}{l}54 \\
62\end{array}$ & $\begin{array}{l}73 \\
71\end{array}$ & $\begin{array}{l}90 \\
86\end{array}$ & $\begin{array}{l}85 \\
79 \\
79\end{array}-x$ & $\begin{array}{l}86 \\
82\end{array}$ & $\begin{array}{l}57 \\
64\end{array}$ & 92 & $\begin{array}{l}70 \\
80\end{array}$ & $\begin{array}{l}50 \\
50\end{array}$ & $\begin{array}{l}90 \\
10\end{array}$ & $\begin{array}{l}35 \\
21\end{array}$ & $\begin{array}{l}38 \\
35 \\
35\end{array}-y$ & $\begin{array}{l}38 \\
27\end{array}$ & $\begin{array}{l}50 \\
35\end{array}-10$ & $\begin{array}{l}32 \\
45\end{array}$ & $\begin{array}{l}51 \\
33\end{array}$ & $\begin{array}{l}52 \\
28\end{array}$ & $\begin{array}{l}45 \\
22\end{array}$ & $\begin{array}{l}50 \\
49\end{array}$ & ${ }_{32}^{67}$ \\
\hline $\begin{array}{l}\text { Ungroupe de produi } \\
\text { Tous les produits } \\
\text { Suoerficie }\end{array}$ & $\begin{array}{r}4 \\
10 \\
90\end{array}$ & $\begin{array}{c}6 \\
94\end{array}$ & $\begin{array}{r}33 \\
6 \\
87\end{array}$ & $\begin{array}{l}19 \\
10 \\
90\end{array}$ & $\begin{array}{r}6 \\
98 \\
92\end{array}$ & $\begin{array}{r}99 \\
12 \\
88\end{array}$ & $\begin{array}{r}99 \\
99 \\
91\end{array}$ & $\begin{array}{r}30 \\
6 \\
94\end{array}$ & $\begin{array}{r}10 \\
98 \\
92\end{array}$ & $\begin{array}{l}1 \\
18 \\
82\end{array}$ & $\begin{array}{r}55 \\
45 \\
54\end{array}$ & $\begin{array}{l}88 \\
12 \\
12\end{array}$ & 48 & $\begin{array}{l}29 \\
36 \\
64 \\
64\end{array}$ & $\begin{array}{l}37 \\
36 \\
64\end{array}$ & $\begin{array}{l}19 \\
46 \\
53\end{array}$ & $\begin{array}{l}28 \\
27 \\
27\end{array}$ & $\begin{array}{l}19 \\
48\end{array}$ & $\begin{array}{l}23 \\
49 \\
49\end{array}$ & $\begin{array}{l}37 \\
41 \\
47\end{array}$ & 46 & 107 \\
\hline $\begin{array}{l}\text { Nombrere d'animaux } \\
\text { Recertes }\end{array}$ & 10 & 0 & 8 & o & o & o & o & 0 & 0 & 0 & 45 & 88 & $33^{-1}$ & $\begin{array}{c}1 \\
0\end{array}$ & : & $\stackrel{\circ}{\circ}$ & $\begin{aligned} 0 \\
2 \\
2\end{aligned}$ & $\begin{array}{cc}0 \\
0 \\
0\end{array}$ & 음 & $\stackrel{0}{3}_{3}$ & 은 & $\stackrel{\circ}{\circ}$ \\
\hline
\end{tabular}


Tableau annexe 7. Catégorie D (PHR) : composition de la catégorie par pays et étiquette, 1986-2007

Part de la catégorie en pourcentage

\begin{tabular}{|c|c|c|c|c|c|c|c|c|c|c|c|c|c|c|c|c|c|c|c|c|c|c|}
\hline $\begin{array}{l}\text { OCDE (USD) } \\
\text { Limites }\end{array}$ & 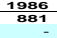 & 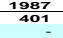 & $\begin{array}{l}\frac{1988}{318} \\
318\end{array}$ & $\begin{array}{l}1589 \\
153\end{array}$ & $\begin{array}{l}1990 \\
93\end{array}$ & $\frac{1991}{24}$ & $\frac{1992}{25}$ & $\begin{array}{l}1993 \\
291\end{array}$ & $\begin{array}{l}1994 \\
407\end{array}$ & $\frac{1995}{472}$ & $\begin{array}{l}1996 \\
437 \\
4\end{array}$ & $\begin{array}{l}\frac{1997}{418} \\
5\end{array}$ & $\begin{array}{c}1998 \\
417 \\
5\end{array}$ & $\begin{array}{l}1999 \\
33 \\
33\end{array}$ & $\begin{array}{c}2000 \\
31 \\
31\end{array}$ & $\begin{array}{c}\frac{201}{65} \\
27\end{array}$ & $\begin{array}{c}202 \\
28 \\
26 \\
26\end{array}$ & $\begin{array}{l}\frac{2003}{652} \\
31\end{array}$ & $\begin{array}{l}2004 \\
\mathbf{6} 67 \\
31\end{array}$ & $\begin{array}{l}2005 \\
717 \\
28\end{array}$ & $\begin{array}{l}2006 \\
820 \\
38\end{array}$ & $\begin{array}{l}\frac{1077}{527} \\
52\end{array}$ \\
\hline 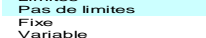 & 100 & $\begin{array}{l}100 \\
100\end{array}$ & $\begin{array}{l}100 \\
100\end{array}$ & $\begin{array}{l}100 \\
100\end{array}$ & $\begin{array}{l}100 \\
100\end{array}$ & $\begin{array}{l}100 \\
100\end{array}$ & $\begin{array}{l}100 \\
100\end{array}$ & $\begin{array}{l}100 \\
100\end{array}$ & $\begin{array}{l}100 \\
100\end{array}$ & $\begin{array}{l}100 \\
100\end{array}$ & $\begin{array}{r}95 \\
100 \\
-\end{array}$ & $\begin{array}{r}95 \\
100 \\
100\end{array}$ & $\begin{array}{r}95 \\
100 \\
100\end{array}$ & $\begin{array}{r}33 \\
68 \\
100\end{array}$ & $\begin{array}{r}31 \\
69 \\
100\end{array}$ & $\begin{array}{r}27 \\
73 \\
100\end{array}$ & $\begin{array}{r}26 \\
70 \\
100\end{array}$ & $\begin{array}{r}31 \\
69 \\
100\end{array}$ & $\begin{aligned} 31 \\
68 \\
100\end{aligned}$ & $\begin{array}{r}28 \\
72 \\
100\end{array}$ & $\begin{array}{l}38 \\
62 \\
86 \\
14\end{array}$ & $\begin{array}{l}52 \\
48 \\
96 \\
4\end{array}$ \\
\hline 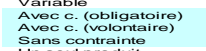 & $\begin{array}{l}2 \\
98\end{array}$ & $\begin{array}{r}4 \\
96\end{array}$ & $\begin{array}{r}7 \\
93\end{array}$ & $\begin{array}{l}12 \\
88\end{array}$ & $\begin{array}{l}26 \\
74\end{array}$ & 100 & $100^{\circ}$ & $100^{\circ}$ & $100^{\circ}$ & 100 & 95 & $\stackrel{95}{5}$ & $\begin{array}{r}95 \\
5\end{array}$ & $\begin{array}{l}6 \overline{7} \\
33\end{array}$ & $\begin{array}{l}69 \\
31\end{array}$ & $\begin{array}{l}73 \\
27\end{array}$ & $\begin{array}{l}74 \\
26\end{array}$ & $\begin{array}{l}10 \\
58 \\
32\end{array}$ & $\begin{array}{l}11 \\
56 \\
33\end{array}$ & $\begin{array}{l}10 \\
61 \\
28\end{array}$ & $\begin{array}{l}14 \\
\text { s. } \\
53 \\
38\end{array}$ & $\begin{aligned} 4 \\
5 \\
44 \\
52\end{aligned}$ \\
\hline $\begin{array}{l}\text { Un seul produit } \\
\text { Un souplup duodul } \\
\text { Tous les produits }\end{array}$ & $\begin{array}{l}58 \\
52\end{array}$ & $\begin{array}{r}4 \\
96 \\
96\end{array}$ & $\begin{array}{l}7 \\
93\end{array}$ & $\begin{array}{l}12 \\
88\end{array}$ & $\begin{array}{l}26 \\
74\end{array}$ & $100^{\circ}$ & 100 & 97 & ${ }_{92}^{\overline{8}}$ & 99 & $\begin{array}{c}15 \\
85 \\
85\end{array}$ & $\begin{array}{c}15 \\
84 \\
84\end{array}$ & $\begin{array}{r}5 \\
15 \\
84\end{array}$ & $\begin{array}{l}33 \\
67 \\
7\end{array}$ & $\begin{array}{l}31 \\
69 \\
-\end{array}$ & $\begin{array}{c}27 \\
73\end{array}$ & $\begin{array}{l}206 \\
74\end{array}$ & $\begin{array}{l}53 \\
53 \\
37\end{array}$ & $\begin{array}{l}52 \\
11 \\
37\end{array}$ & $\begin{array}{l}54 \\
10 \\
36\end{array}$ & $\begin{array}{r}47 \\
44 \\
44\end{array}$ & $\begin{array}{l}38 \\
39 \\
23\end{array}$ \\
\hline 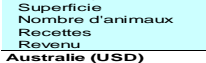 & $\begin{array}{l}42 \\
58\end{array}$ & $\begin{array}{r}96 \\
4\end{array}$ & $\stackrel{93}{7}$ & $\begin{array}{l}88 \\
12\end{array}$ & $\begin{array}{l}74 \\
26 \\
-\end{array}$ & $100^{\circ}$ & 100 & $\begin{array}{r}7 \\
93 \\
9-\end{array}$ & $\begin{array}{r}8 \\
8 \\
92\end{array}$ & $\begin{array}{r}9 \\
91 \\
\end{array}$ & $\begin{array}{l}15 \\
85 \\
\end{array}$ & $\begin{array}{l}16 \\
84 \\
4\end{array}$ & $\begin{array}{l}16 \\
84 \\
84\end{array}$ & 100 & $100^{\circ}$ & $100^{\circ}$ & 100 & $\begin{array}{r}37 \\
36 \\
27 \\
0 \\
\end{array}$ & $\begin{array}{r}37 \\
34 \\
27 \\
11 \\
11\end{array}$ & $\begin{array}{r}36 \\
41 \\
23 \\
0 \\
3 \\
\end{array}$ & $\begin{array}{r}30 \\
36 \\
19 \\
14 \\
\end{array}$ & $\begin{array}{r}19 \\
35 \\
45 \\
4 \\
-\end{array}$ \\
\hline $\begin{array}{l}\text { Limites } \\
\text { Laide limites } \\
\text { Fix de }\end{array}$ & & & & & & & & & & & & & & & & & & $\begin{array}{l}100 \\
100\end{array}$ & $\begin{array}{l}100 \\
100\end{array}$ & $\begin{array}{l}100 \\
100\end{array}$ & & \\
\hline 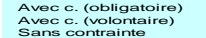 & & & & & & & & & & & & & & & & & & & & & & \\
\hline 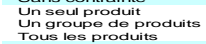 & & & & & & & & & & & & & & & & & & $\begin{array}{l}7100 \\
100\end{array}$ & $\begin{array}{l}100 \\
100 \\
-\end{array}$ & $\begin{array}{l}100 \\
100 \\
-\end{array}$ & & \\
\hline 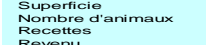 & & & & & & & & & & & & & & & & & & 100 & 100 & 100 & & \\
\hline 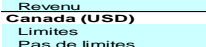 & - & - & - & . & & & & & & & & & & & & & & 10 & - & - & $\begin{array}{l}117 \\
100\end{array}$ & $\begin{array}{c}584 \\
100\end{array}$ \\
\hline 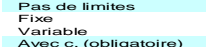 & & & & & & & & & & & & & & & & & & & & & 100 & $\begin{array}{l}89 \\
11\end{array}$ \\
\hline 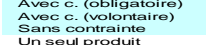 & & & & & & & & & & & & & & & & & & & & & 100 & $100^{\circ}$ \\
\hline 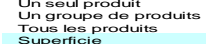 & & & & & & & & & & & & & & & & & & & & & $100^{\circ}$ & $\begin{array}{l}899 \\
11\end{array}$ \\
\hline $\begin{array}{l}\text { Nombrod d'animaux } \\
\text { Recertes } \\
\text { Reventu } \\
\text { Revent }\end{array}$ & & & & & & & & & & & & & & & & & & & & & $100^{\circ}$ & $\begin{array}{l}899 \\
11 \\
\end{array}$ \\
\hline 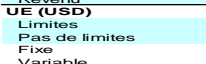 & & & & & & & & & & & & & & & & & & & & & & \\
\hline 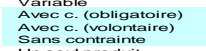 & & & & & & & & & & & & & & & & & & & & & & \\
\hline $\begin{array}{l}\text { Un seiul produit } \\
\text { Un groupeduits } \\
\text { Tous les produits }\end{array}$ & & & & & & & & & & & & & & & & & & & & & & \\
\hline 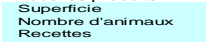 & & & & & & & & & & & & & & & & & & & & & & \\
\hline 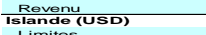 & 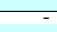 & 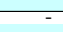 & - & 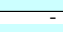 & & 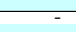 & & & & & 22 & 22 & 22 & 22 & 21 & 18 & 120 & 27 & 30 & 35 & 35 & 39 \\
\hline 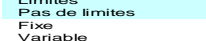 & & & & & & & & & & & $\begin{array}{l}100 \\
100\end{array}$ & $\begin{array}{l}100 \\
100\end{array}$ & $\begin{array}{l}100 \\
100\end{array}$ & $\begin{array}{l}100 \\
100\end{array}$ & $\begin{array}{l}100 \\
100\end{array}$ & $\begin{array}{l}100 \\
100\end{array}$ & $\begin{array}{l}100 \\
100\end{array}$ & $\begin{array}{l}100 \\
100\end{array}$ & $\begin{array}{l}100 \\
100\end{array}$ & $\begin{array}{l}100 \\
100\end{array}$ & $\begin{array}{l}100 \\
100\end{array}$ & $\begin{array}{l}100 \\
100\end{array}$ \\
\hline 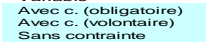 & & & & & & & & & & & & & & & & & & & & & & \\
\hline 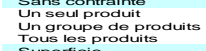 & & & & & & & & & & & $\begin{array}{l}100 \\
100\end{array}$ & $\begin{array}{l}700 \\
100 \\
100\end{array}$ & $\begin{array}{l}100 \\
100\end{array}$ & $\begin{array}{l}100 \\
100\end{array}$ & $\begin{array}{l}100 \\
100\end{array}$ & $\begin{array}{l}7100 \\
100\end{array}$ & $\begin{array}{l}100 \\
100\end{array}$ & $\begin{array}{l}7100 \\
100\end{array}$ & $\begin{array}{l}100 \\
100\end{array}$ & $\begin{array}{l}7100 \\
100\end{array}$ & $\begin{array}{l}100 \\
100 \\
\end{array}$ & $\begin{array}{l}100 \\
100\end{array}$ \\
\hline 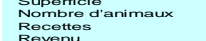 & & & & & & & & & & & 100 & 100 & 100 & 100 & 100 & 100 & 100 & 100 & 100 & 100 & 100 & 100 \\
\hline 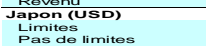 & & & & & & & & & & & & & & & & & & & & & & \\
\hline 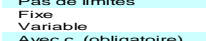 & & & & & & & & & & & & & & & & & & & & & & \\
\hline 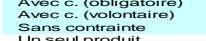 & & & & & & & & & & & & & & & & & & & & & & \\
\hline 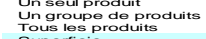 & & & & & & & & & & & & & & & & & & & & & & \\
\hline 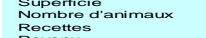 & & & & & & & & & & & & & & & & & & & & & & \\
\hline 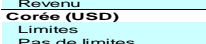 & & & & & & - & & & & & & & & - & & & & & & & & \\
\hline $\begin{array}{l}\text { Pas de limites } \\
\text { Fixeriable } \\
\text { Variale }\end{array}$ & & & & & & & & & & & & & & & & & & & & & & \\
\hline 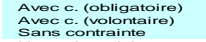 & & & & & & & & & & & & & & & & & & & & & & \\
\hline 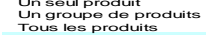 & & & & & & & & & & & & & & & & & & & & & & \\
\hline $\begin{array}{l}\text { Superifieieanimaux } \\
\text { Nomborles } \\
\text { Revenu }\end{array}$ & & & & & & & & & & & & & & & & & & & & & & \\
\hline
\end{tabular}

ANALYSE DE LA COMPOSITION DU SOUTIEN AUX PRODUCTEURS : NOUVEAUX OUTILS ET MÉTHODES ๑ OECD 2010 
50 - ANNEXE 2. TABLEAUX ANNEXES

Tableau annexe 7. Catégorie D (PHR) : composition de la catégorie par pays et étiquette, 1986-2007 (suite)

Part de la catégorie en pourcentage

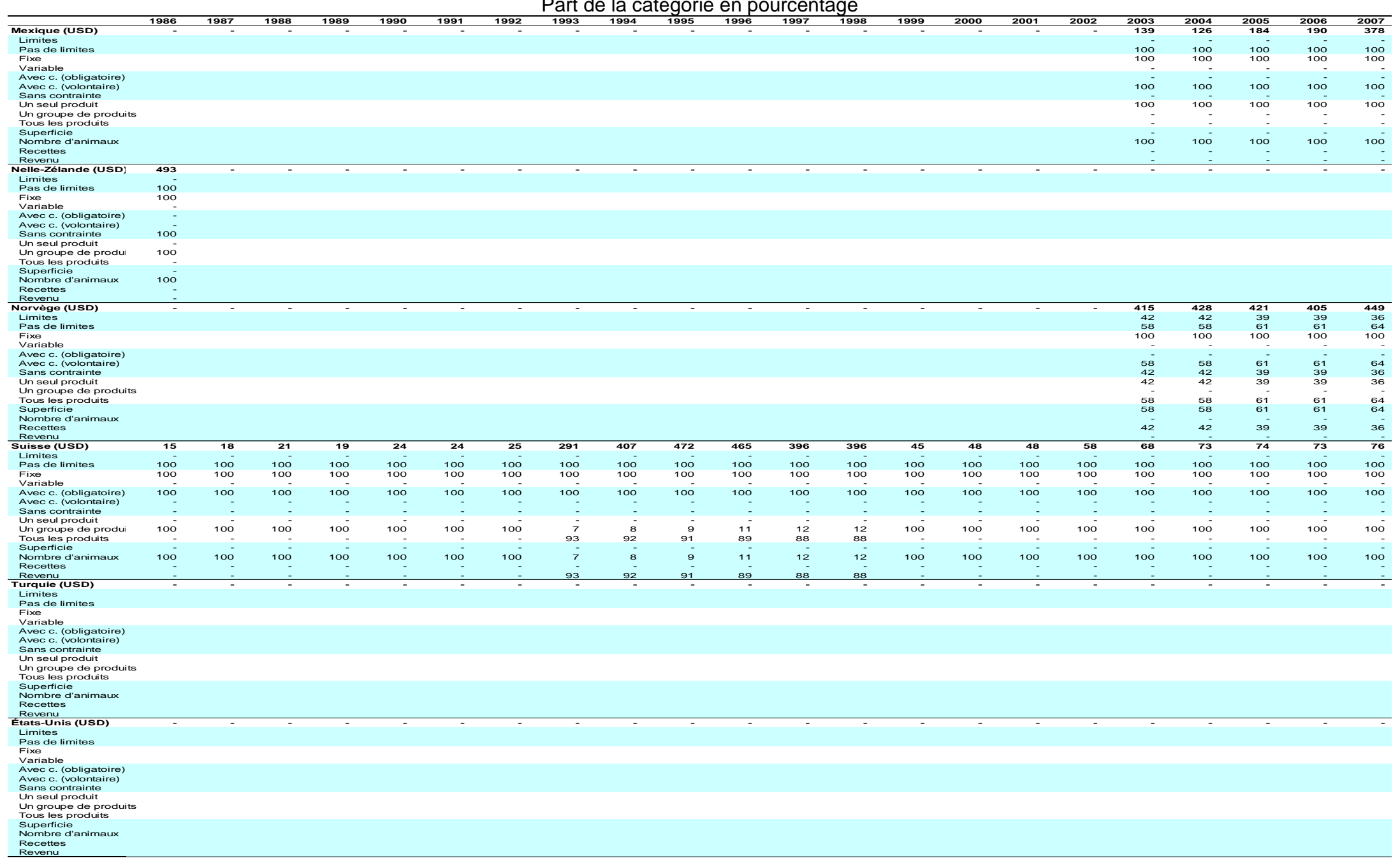


Tableau annexe 8. Catégorie E (PHNR) : composition de la catégorie par pays et étiquette, 1986-2007

\begin{tabular}{|c|c|c|c|c|c|c|c|c|c|c|c|c|c|c|c|c|c|c|c|c|c|c|}
\hline OCDE (USD) & $\begin{array}{l}\frac{1986}{21997} \\
227\end{array}$ & $\begin{array}{l}19887 \\
1943 \\
133\end{array}$ & $\begin{array}{l}\frac{1988}{2101} \\
2108\end{array}$ & $\begin{array}{l}\frac{1988}{2160} \\
2160\end{array}$ & $\begin{array}{l}1990 \\
1809 \\
162\end{array}$ & $\begin{array}{l}1991 \\
17 \mathbf{1}^{2}\end{array}$ & $\begin{array}{l}1992 \\
2334\end{array}$ & $\begin{array}{l}1993 \\
2066\end{array}$ & $\begin{array}{l}19994 \\
3394 \\
\end{array}$ & $\begin{array}{l}\frac{1995}{2995} \\
\end{array}$ & $\begin{array}{l}\frac{1996}{8358} \\
8359\end{array}$ & $\begin{array}{l}1997 \\
8488 \\
\end{array}$ & 10908 & $\begin{array}{l}19999 \\
13830\end{array}$ & $\begin{array}{l}12000 \\
14641\end{array}$ & 13001 & $\frac{2002}{13153}$ & $\begin{array}{l}136033 \\
13649\end{array}$ & $\frac{2004}{17729}$ & $\begin{array}{l}38 \text { (2019 } \\
3819\end{array}$ & $\begin{array}{l}\frac{2006}{53642} \\
5.96\end{array}$ & $\begin{array}{l}\frac{2007}{60634} \\
6092\end{array}$ \\
\hline $\begin{array}{l}\text { Limes limites } \\
\text { Fis de lie lie } \\
\text { Variable }\end{array}$ & $\begin{array}{l}27 \\
73 \\
95 \\
95\end{array}$ & $\begin{array}{l}13 \\
80 \\
90 \\
10\end{array}$ & $\begin{array}{l}88 \\
89 \\
11 \\
11\end{array}$ & 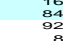 & $\begin{array}{l}75 \\
75 \\
90\end{array}$ & $\begin{aligned} \frac{37}{30} \\
100 \\
0\end{aligned}$ & $\begin{array}{c}53 \\
57 \\
3 \\
3\end{array}$ & $\begin{array}{c}50 \\
5 \% \\
3 \\
3\end{array}$ & $\begin{array}{l}73 \\
28 \\
98 \\
2\end{array}$ & $\begin{array}{l}319 \\
67 \\
33 \\
3.0\end{array}$ & $\begin{array}{l}73 \\
\substack{73 \\
00 \\
10}\end{array}$ & $\begin{array}{l}86 \\
14 \\
99 \\
91\end{array}$ & $\begin{array}{l}91 \\
92 \\
72 \\
28\end{array}$ & $\begin{array}{l}86 \\
16 \\
160 \\
40\end{array}$ & $\begin{array}{l}82 \\
18 \\
61 \\
39\end{array}$ & 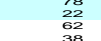 & $\begin{array}{l}25 \\
33 \\
17 \\
17\end{array}$ & $\begin{array}{l}24 \\
23 \\
93\end{array}$ & $\begin{array}{l}80 \\
79 \\
74\end{array}$ & $\begin{array}{l}89 \\
17 \\
17 \\
13\end{array}$ & $\begin{array}{l}93 \\
93 \\
96 \\
4\end{array}$ & $\begin{array}{l}92 \\
98 \\
97 \\
3\end{array}$ \\
\hline 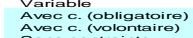 & & & & & 25 & 23 & & ${ }^{3} 0^{3}$ & $31^{2}$ & & $\begin{array}{l}10 \\
62\end{array}$ & 74 & $\begin{array}{l}28 \\
82 \\
32\end{array}$ & $\begin{array}{l}40 \\
85\end{array}$ & $\begin{array}{l}39 \\
77\end{array}$ & $\begin{array}{l}38 \\
72 \\
72\end{array}$ & $\begin{array}{l}17 \\
60 \\
-10\end{array}$ & 50 & $\begin{array}{l}26 \\
69\end{array}$ & $\begin{array}{l}13 \\
79 \\
9\end{array}$ & $\begin{array}{l}34 \\
86\end{array}$ & $\begin{array}{r}35^{3} \\
1\end{array}$ \\
\hline 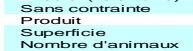 & 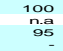 & 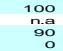 & 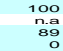 & $\begin{array}{l}84 \\
\text { na: } \\
\text { 92 } \\
\text { o }\end{array}$ & $\begin{array}{l}75 \\
7 . .5 \\
99 \\
0\end{array}$ & $\begin{array}{l}77 \\
\text { n.a } \\
\text { 9o }\end{array}$ & 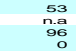 & $\begin{array}{l}50 \\
\text { noa } \\
90 \\
0\end{array}$ & 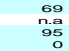 & $\begin{array}{c}99 \\
99 \\
93\end{array}$ & $\begin{aligned} 38 \\
7.7 \\
90\end{aligned}$ & $\begin{array}{l}26 \\
\text { n.a. } \\
\text { sog } \\
\text { o }\end{array}$ & $\begin{array}{l}18 \\
\text { n... } \\
\text { 9o } \\
\text { o }\end{array}$ & $\begin{array}{l}15 \\
\text { na: } \\
90 \\
90\end{array}$ & $\begin{array}{l}23 \\
\text { nat } \\
90 \\
0\end{array}$ & $\begin{array}{l}28 \\
\text { n.a } \\
95 \\
0\end{array}$ & $\begin{array}{l}40 \\
\text { nna. } \\
93 \\
0\end{array}$ & 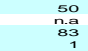 & $\begin{array}{ll}3.1 \\
\text { n.a } \\
93\end{array}$ & $\begin{array}{l}21 \\
\text { n.a. } \\
93 \\
0\end{array}$ & $\begin{array}{l}1.4 \\
\text { nog } \\
\text { 9. } \\
\text { o }\end{array}$ & $\begin{array}{l}14 \\
\text { n. } \\
9.5 \\
\text { s. }\end{array}$ \\
\hline 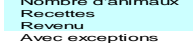 & ${ }_{68}^{5}$ & $\begin{array}{l}10 \\
77\end{array}$ & ${ }_{81}^{1 \overline{1}}$ & $78^{8}$ & $7 \frac{1}{4}$ & $7 \overline{2}^{2}$ & $\underset{49}{4}$ & $4_{4}^{6}$ & $\frac{5}{54}$ & $\frac{0}{9}$ & $\begin{array}{l}\frac{1}{1} \\
8^{2}\end{array}$ & $\begin{array}{c}0 \\
0 \\
08 \\
98\end{array}$ & $\begin{array}{c}0 \\
0 \\
9 \\
9 \\
9\end{array}$ & $\begin{aligned} 0 \\
9 \\
94\end{aligned}$ & $\begin{array}{r}3 \\
3 \\
88 \\
8\end{array}$ & $\begin{array}{c}\frac{3}{3} \\
85\end{array}$ & $\begin{array}{c}0 \\
\frac{9}{3} \\
75\end{array}$ & $\begin{array}{c}13 \\
\frac{13}{3} \\
64\end{array}$ & $\begin{array}{rl}3 & 3 \\
1 \\
69\end{array}$ & $\begin{array}{c}0 \\
6 \\
5 \\
58\end{array}$ & 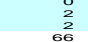 & $\sum_{01}^{2}$ \\
\hline 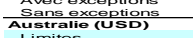 & $\begin{array}{l}32 \\
120\end{array}$ & $\begin{array}{l}23 \\
196\end{array}$ & $\begin{array}{l}19 \\
226\end{array}$ & $\frac{24}{162}$ & $\begin{array}{l}26 \\
9\end{array}$ & $\begin{array}{l}25 \\
37 \\
37\end{array}$ & $\begin{array}{c}51 \\
51 \\
91\end{array}$ & $\begin{array}{c}56 \\
125 \\
125 \\
\end{array}$ & $\begin{array}{l}36 \\
181 \\
136\end{array}$ & $\begin{array}{l}38 \\
209\end{array}$ & $\begin{array}{r}13 \\
197 \\
197\end{array}$ & $\frac{2}{122}$ & 100 & $\begin{array}{l}6 \\
89\end{array}$ & $\frac{12}{244}$ & $\begin{array}{l}15 \\
357\end{array}$ & $\begin{array}{l}\frac{25}{544} \\
554\end{array}$ & $\begin{array}{l}\frac{36}{521} \\
\frac{521}{4}\end{array}$ & $\begin{array}{r}31 \\
400 \\
400\end{array}$ & $\begin{array}{l}\frac{42}{595} \\
95\end{array}$ & $\begin{array}{l}34 \\
580\end{array}$ & $\begin{array}{l}39 \\
930 \\
\end{array}$ \\
\hline 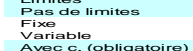 & $\begin{array}{l}100 \\
100\end{array}$ & $\begin{array}{l}100 \\
100\end{array}$ & $\begin{array}{l}100 \\
100\end{array}$ & $\begin{array}{l}100 \\
100\end{array}$ & $\begin{array}{l}100 \\
100\end{array}$ & $\begin{array}{r}100 \\
85 \\
15\end{array}$ & $\begin{aligned} \begin{array}{r}100 \\
39 \\
65 \\
65\end{array} & \end{aligned}$ & $\begin{aligned} \begin{array}{r}100 \\
44 \\
56\end{array} & \end{aligned}$ & $\begin{array}{r}100 \\
57 \\
43\end{array}$ & $\begin{aligned} \begin{array}{r}100 \\
50 \\
50\end{array} & \end{aligned}$ & $\begin{aligned} \begin{array}{r}100 \\
39 \\
61\end{array} & \\
61 & \end{aligned}$ & $\begin{array}{r}100 \\
38 \\
70\end{array}$ & 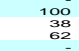 & $\begin{array}{r}\begin{array}{r}100 \\
38 \\
70\end{array} \\
70\end{array}$ & $\begin{array}{r}100 \\
92 \\
92\end{array}$ & $\begin{aligned} \begin{array}{r}100 \\
6 \\
94\end{array} & \end{aligned}$ & $\begin{array}{r}100 \\
14 \\
86\end{array}$ & $\begin{array}{r}100 \\
17 \\
83\end{array}$ & $\begin{aligned} & \begin{array}{r}100 \\
21 \\
79\end{array} \\
& 79\end{aligned}$ & 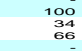 & $\begin{array}{c}100 \\
\substack{100 \\
57 \\
57}\end{array}$ & $\begin{array}{c}100 \\
68 \\
38\end{array}$ \\
\hline 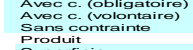 & coo & roo & 售 & rac & 然o & noo & roo & ${ }_{\substack{100 \\
\text { na. }}}$ & $\underset{\substack{100 \\
\text { na. }}}{10}$ & $\underset{\substack{100 \\
\text { na. }}}{20}$ & roo & $\underset{\substack{100 \\
n, a}}{10}$ & $\begin{array}{c}100 \\
\text { no. }\end{array}$ & $\begin{array}{l}100 \\
\text { na. }\end{array}$ & roo & $\begin{array}{c}100 \\
\text { na }\end{array}$ & roo & $\begin{array}{c}100 \\
\text { noa }\end{array}$ & coo & $\begin{array}{l}100 \\
\text { na. }\end{array}$ & roo & roo \\
\hline 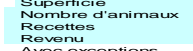 & $100^{\circ}$ & $100^{\circ}$ & 100 & 100 & 100 & 100 & 100 & 100 & 100 & 100 & $100^{\circ}$ & 100 & $100^{\circ}$ & 100 & $\begin{array}{l}39 \\
61 \\
1\end{array}$ & $\begin{array}{l}32 \\
68 \\
62\end{array}$ & $\begin{array}{l}22 \\
78\end{array}$ & $\begin{array}{l}27 \\
73 \\
73\end{array}$ & $\begin{array}{l}42 \\
58 \\
58\end{array}$ & $\begin{array}{l}30 \\
70 \\
70\end{array}$ & 202 & 79 \\
\hline 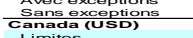 & $100^{\circ}$ & $100^{\circ}$ & 100 & 100 & 100 & 100 & $100^{\circ}$ & $100^{\circ}$ & 100 & $\frac{100}{905}$ & $\frac{100}{825}$ & $\frac{100^{-}}{3}$ & $100^{\circ}$ & $\begin{array}{r}17 \\
83 \\
0\end{array}$ & $\begin{aligned} 12 \\
\text { sas } \\
544\end{aligned}$ & $\begin{array}{l}22 \\
\mathbf{5 2 8} \\
\mathbf{5 2 4}\end{array}$ & $\begin{array}{r}41 \\
\mathbf{5 8 9} \\
\mathbf{5 8 8}\end{array}$ & $\begin{array}{c}\frac{30}{370} \\
534\end{array}$ & 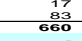 & $\begin{array}{l}15 \\
\frac{15}{432} \\
\frac{1}{432}\end{array}$ & $\begin{array}{l}14 \\
\frac{14}{776} \\
776\end{array}$ & $\begin{array}{c}99 \\
983 \\
983\end{array}$ \\
\hline 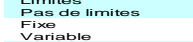 & & & & & & & & & & $\begin{array}{r}100 \\
2 \\
98\end{array}$ & $\begin{array}{c}100 \\
14 \\
86\end{array}$ & $\begin{array}{r}100 \\
28 \\
72\end{array}$ & $\begin{array}{l}100^{\circ} \\
100\end{array}$ & $\begin{array}{l}100^{\circ} \\
100\end{array}$ & $\begin{array}{l}100 \\
100\end{array}$ & $\begin{array}{l}100 \\
100\end{array}$ & $\begin{array}{l}100^{\circ} \\
100\end{array}$ & $\begin{array}{l}100^{\circ} \\
100\end{array}$ & $\begin{array}{l}100^{\circ} \\
100\end{array}$ & $\begin{array}{l}100 \\
100\end{array}$ & $\begin{array}{l}94 \\
96 \\
63 \\
37\end{array}$ & $\begin{array}{c}36 \\
54 \\
56 \\
46\end{array}$ \\
\hline 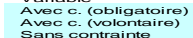 & & & & & & & & & & & & & & & & & & & & & & \\
\hline 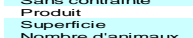 & n.a & n.a & n.a & n.a & n.a & n.a & n.a & n.a & n.a & nag & $\begin{array}{l}\text { lo } \\
\text { nas } \\
93\end{array}$ & $\begin{array}{l}100 \\
72 \\
72\end{array}$ & n.a & n.a & $\begin{array}{l}n_{4} \\
\text { nat } \\
45\end{array}$ & $\begin{array}{l}n_{n a} \\
\text { nas }\end{array}$ & $\begin{array}{l}\text { na } \\
\text { nas } \\
\text { s. }\end{array}$ & na. & n.a & n.a & $\begin{array}{l}1.0 \\
\text { nis } \\
\text { is }\end{array}$ & 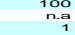 \\
\hline $\begin{array}{l}\text { Nombred d'animaux } \\
\text { neverts } \\
\text { Revanu }\end{array}$ & & & & & & & & & & $2^{2}$ & $i$ & 28 & $100^{\circ}$ & 100 & 55 & si & $65^{\circ}$ & $\begin{array}{l}14 \\
8 \mathrm{~s} \\
6\end{array}$ & $\begin{array}{l}70 \\
27 \\
4\end{array}$ & 89 & $\begin{array}{l}1 \\
84\end{array}$ & $\begin{array}{l}35 \\
63 \\
63\end{array}$ \\
\hline 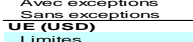 & & & & 350 & 407 & 390 & 091 & 1030 & 1050 & $\frac{100}{35}$ & $\frac{100}{29}$ & $\frac{100}{25}$ & $\frac{100}{24}$ & $\frac{100}{22}$ & $\frac{100}{18}$ & 100 & 100 & $100^{\circ}$ & 100 & $\frac{100}{767}$ & $\frac{100}{8265}$ & 430 \\
\hline $\begin{array}{l}\text { Limitios limites } \\
\text { Fas de limites } \\
\text { File }\end{array}$ & & & & $\begin{array}{l}100 \\
100\end{array}$ & $\begin{array}{l}100 \\
100\end{array}$ & $\begin{array}{l}100 \\
100\end{array}$ & $\begin{array}{l}100 \\
100\end{array}$ & $\begin{array}{l}100 \\
100\end{array}$ & $\begin{array}{l}100 \\
100\end{array}$ & $\begin{array}{l}100 \\
100\end{array}$ & $\begin{array}{l}100 \\
100\end{array}$ & $\begin{array}{l}100 \\
100\end{array}$ & $\begin{array}{l}100 \\
100\end{array}$ & $\begin{array}{l}100 \\
100\end{array}$ & $\begin{array}{l}100 \\
100\end{array}$ & $\begin{array}{l}100 \\
100\end{array}$ & $\begin{array}{l}100 \\
100\end{array}$ & $\begin{array}{l}100 \\
100\end{array}$ & $\begin{array}{l}100 \\
100\end{array}$ & $\begin{array}{l}100 \\
100\end{array}$ & $\begin{array}{l}100 \\
100\end{array}$ & $\begin{array}{l}100 \\
100 \\
100\end{array}$ \\
\hline 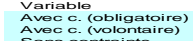 & & & & 100 & 100 & $10^{-}$ & $100^{\circ}$ & 100 & $100^{-}$ & 100 & 100 & 100 & $100^{\circ}$ & 100 & 100 & 100 & 100 & 100 & 100 & 100 & $100^{\circ}$ & 100 \\
\hline 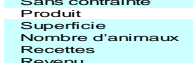 & n.a & n.a & n.a & $\begin{array}{l}\text { nao } \\
\text { roo } \\
\text { - }\end{array}$ & $\begin{array}{l}\text { nat } \\
\text { ioo }\end{array}$ & no & $\begin{array}{l}\text { nat } \\
\text { 10o }\end{array}$ & $\begin{array}{l}\text { nas } \\
\text { ioo }\end{array}$ & $\begin{array}{l}\text { nat } \\
\text { ioo }\end{array}$ & na. & $\begin{array}{l}\text { nat } \\
\text { ioo }\end{array}$ & nas & $\begin{array}{l}\text { nata } \\
\text { 10o }\end{array}$ & nat & nas & $\begin{array}{l}\text { nat } \\
\text { 10o }\end{array}$ & $\begin{array}{l}\text { nat } \\
\text { 10o }\end{array}$ & $\begin{array}{l}\text { nat } \\
\text { roo }\end{array}$ & $\begin{array}{l}\text { nat } \\
\text { roo }\end{array}$ & nas & $\begin{array}{l}\text { nata } \\
\text { 10o }\end{array}$ & $\begin{array}{c}\text { nat } \\
100\end{array}$ \\
\hline 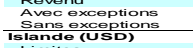 & & 2 & 2 & $\frac{100}{2}$ & $\frac{100}{2}$ & $\frac{100}{2}$ & 100 & 100 & 100 & $100^{\circ}$ & 100 & $\frac{100}{1}$ & $100^{\circ}$ & 100 & 100 & $\frac{100}{10}$ & $100^{\circ}$ & $100^{\circ}$ & $100^{\circ}$ & $\begin{array}{r}50 \\
50 \\
1 \\
1\end{array}$ & $\begin{array}{l}69 \\
31 \\
31\end{array}$ & $\begin{array}{l}64 \\
36 \\
-1\end{array}$ \\
\hline $\begin{array}{l}\text { Limites limites } \\
\text { Fas de lie le } \\
\text { Variable }\end{array}$ & & $\begin{array}{l}100 \\
100\end{array}$ & 100 & 100 & $\begin{array}{l}100 \\
100\end{array}$ & 100 & $\begin{array}{l}100 \\
100\end{array}$ & $\begin{array}{l}100 \\
100\end{array}$ & $\begin{array}{l}100 \\
100\end{array}$ & & $\begin{array}{l}100 \\
100\end{array}$ & $\begin{array}{l}100 \\
100\end{array}$ & $\begin{array}{l}100 \\
100\end{array}$ & $\begin{array}{l}100 \\
100\end{array}$ & $\begin{array}{l}100 \\
100\end{array}$ & $\begin{array}{l}100 \\
100\end{array}$ & $\begin{array}{l}100 \\
100\end{array}$ & $\begin{array}{l}100 \\
100\end{array}$ & $\begin{array}{l}100 \\
100\end{array}$ & $\begin{array}{l}100 \\
100\end{array}$ & & \\
\hline 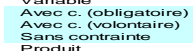 & & 100 & 100 & 100 & 100 & 100 & 100 & 100 & 100 & & 100 & 100 & 100 & 100 & 100 & 100 & 100 & 100 & 100 & 100 & & \\
\hline 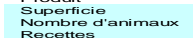 & & 100 & 100 & 100 & 100 & 100 & 100 & 100 & 100 & a & 100 & 100 & 100 & 100 & 100 & 100 & 100 & 100 & 100 & 100 & mat & \\
\hline 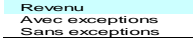 & & 100 & 100 & 100 & 100 & 100 & $100^{-}$ & 100 & 100 & & $100^{\circ}$ & $100^{-}$ & $100^{\circ}$ & 100 & 100 & 100 & 100 & 100 & 100 & 100 & & \\
\hline 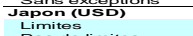 & 1484 & 1491 & 1704 & 1647 & 1192 & 1275 & 1152 & & 732 & 949 & 226 & 1099 & 884 & 025 & & ${ }_{18}^{508}$ & & & $\begin{array}{l}495 \\
11 \\
11\end{array}$ & & & \\
\hline $\begin{array}{l}\text { Pas de limites } \\
\text { Fixiable } \\
\text { variable }\end{array}$ & $\begin{array}{l}100 \\
100\end{array}$ & $\begin{array}{l}100 \\
100\end{array}$ & $\begin{array}{l}100 \\
100\end{array}$ & $\begin{array}{l}100 \\
100\end{array}$ & $\begin{array}{l}100 \\
100\end{array}$ & 100 & $\begin{array}{l}100 \\
100\end{array}$ & $\begin{array}{l}100 \\
100\end{array}$ & $\begin{array}{l}100 \\
100\end{array}$ & $\begin{array}{l}100 \\
100\end{array}$ & $\begin{array}{l}100 \\
100\end{array}$ & $\begin{array}{l}100 \\
100\end{array}$ & $\begin{array}{l}100 \\
100\end{array}$ & $\begin{array}{l}100 \\
100\end{array}$ & $\begin{array}{l}\text { so } \\
100 \\
100\end{array}$ & $\begin{aligned} & 82 \\
100 & -1\end{aligned}$ & 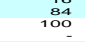 & $\begin{array}{r}88 \\
100\end{array}$ & $\begin{array}{r}88 \\
100 \\
100\end{array}$ & $\begin{array}{l}88 \\
100 \\
100\end{array}$ & $\begin{array}{c}38 \\
100 \\
100\end{array}$ & 100 \\
\hline 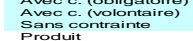 & 100 & $100^{\circ}$ & 10o & 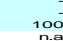 & roo & 10o & 100 & 100 & 100 & n.a & roo & 100 & $\begin{array}{c}100 \\
\text { na }\end{array}$ & roo & roo & ${ }_{n}^{100}$ & $\begin{array}{c}100 \\
\text { na }\end{array}$ & $\begin{array}{c}100 \\
\text { na }\end{array}$ & roo & 100 & 100 & $\begin{array}{c}37 \\
63 \\
n \\
n a\end{array}$ \\
\hline 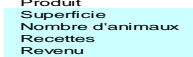 & $\begin{array}{l}\text { naa } \\
100\end{array}$ & $\begin{array}{l}\text { naa } \\
100\end{array}$ & $\begin{array}{l}\text { naa } \\
\text { noo }\end{array}$ & noo & $\begin{array}{l}\text { n.a } \\
100\end{array}$ & noa & $\begin{array}{l}\text { naa } \\
100\end{array}$ & $\begin{array}{l}\text { naa } \\
\text { 10o }\end{array}$ & $\begin{array}{l}\text { n.a } \\
100\end{array}$ & $\begin{array}{l}\text { naa } \\
100\end{array}$ & $\begin{array}{l}\text { naa } \\
100\end{array}$ & $\begin{array}{l}\text { naa } \\
100\end{array}$ & $\begin{array}{l}\text { nat } \\
\text { 10o }\end{array}$ & na & nat & $\begin{array}{l}\text { na. } \\
\text { roo }\end{array}$ & noo & $\begin{array}{l}\text { naa } \\
\text { 10o }\end{array}$ & noo & $\begin{array}{l}\text { naa } \\
\text { 10o }\end{array}$ & $\begin{array}{l}\text { na. } \\
\text { roo }\end{array}$ & noa \\
\hline 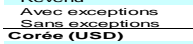 & 100 & ${ }^{100}$ & ${ }^{100}$ & 100 & 100 & 100 & 100 & 100 & 100 & 100 & ${ }^{100}=$ & 100 & 100 & 100 & $\begin{array}{l}80 \\
20 \\
-2\end{array}$ & $\begin{array}{l}82 \\
18 \\
-\end{array}$ & $\begin{array}{l}84 \\
16 \\
16 \\
-\end{array}$ & $\begin{array}{l}89 \\
11 \\
-\end{array}$ & $\begin{array}{r}89 \\
11 \\
8\end{array}$ & 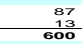 & $\begin{array}{l}\frac{87}{73} \\
790 \\
\end{array}$ & $\begin{array}{c}55 \\
795 \\
799 \\
-\end{array}$ \\
\hline $\begin{array}{l}\text { Limites ilmites } \\
\text { Fise de diable } \\
\text { variable }\end{array}$ & & & & & & & & & & & & & & & & & & & $\begin{array}{l}100 \\
100\end{array}$ & $\begin{array}{l}100 \\
100\end{array}$ & $\begin{array}{l}100 \\
100\end{array}$ & $\begin{array}{l}100 \\
100\end{array}$ \\
\hline 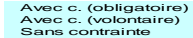 & & & & & & & & & & & & & & & & & & & & & & \\
\hline 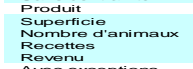 & n.a & n.a & n.a & n.a & n.a & p.a & n.a & i.a. & n.a & n.a & a & n.a & n.a & a. & n.a & n.a & n.a & n.a & $\begin{array}{l}\text { na } \\
\text { noo }\end{array}$ & $\begin{array}{l}\text { naa } \\
\text { 10o }\end{array}$ & $\begin{array}{c}\text { na } \\
\text { noo }\end{array}$ & $\begin{array}{c}n \text { na } \\
\text { noo }\end{array}$ \\
\hline 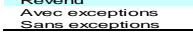 & & & & & & & & & & & & & & & & & & & $100^{\circ}$ & $100^{-}$ & $100^{\circ}$ & 100 \\
\hline
\end{tabular}


$\mathbf{5 2}$ - ANNEXE 2. TABLEAUX ANNEXES

Tableau annexe 8. Catégorie E (PHNR) : composition de la catégorie par pays et étiquette, 1986-2007 (suite)

Part de la catégorie en pourcentage

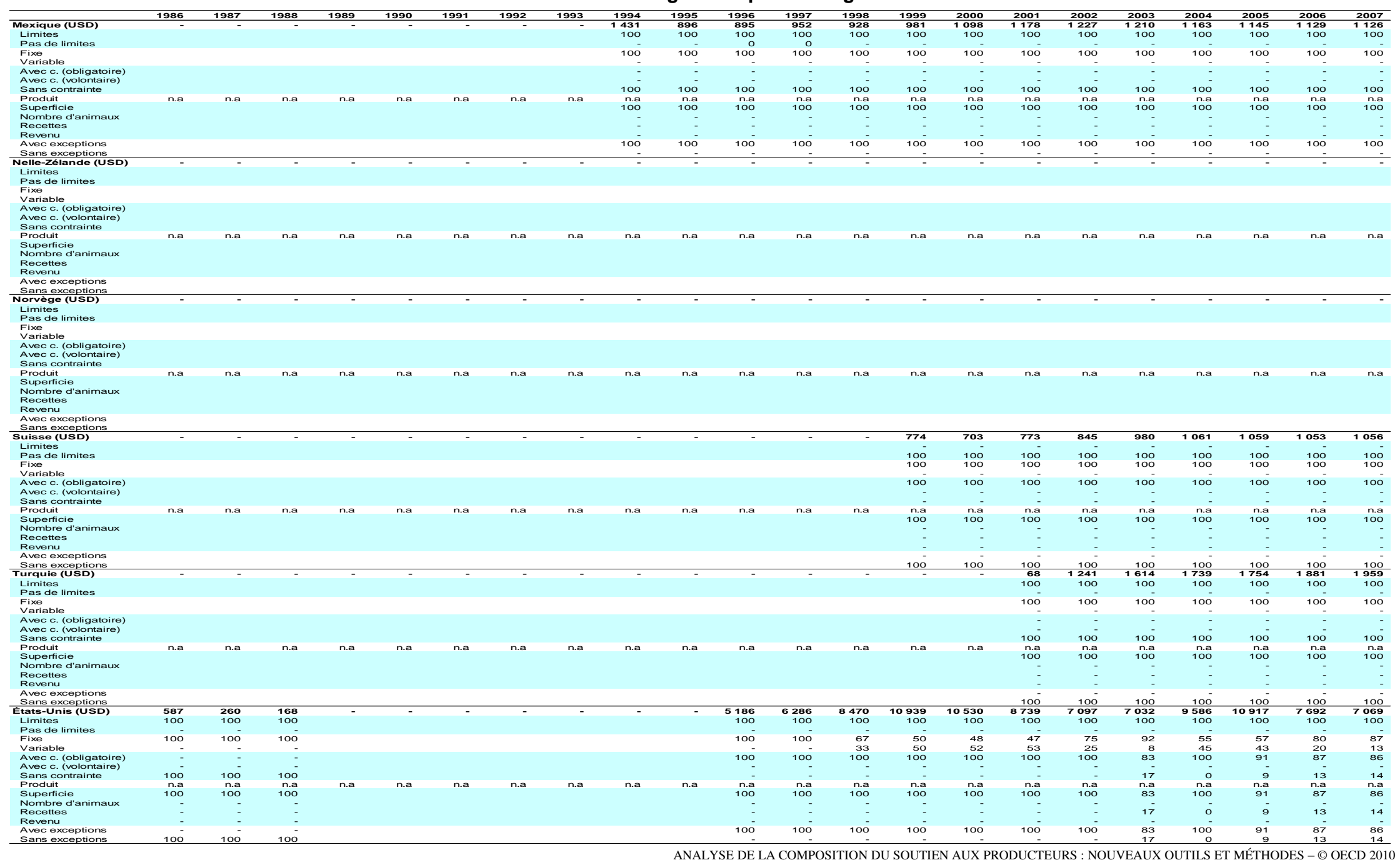


Tableau annexe 9. Catégorie F (PN) : composition de la catégorie par pays et étiquette, 1986-2007

\begin{tabular}{|c|c|c|c|c|c|c|c|c|c|c|c|c|c|c|c|c|c|c|c|c|c|c|}
\hline \multicolumn{23}{|c|}{ Part de la catégorie en pourcentage } \\
\hline OCDE (USD) & $\frac{1986}{321}$ & $\frac{1987}{913}$ & $\frac{1988}{1571}$ & $\frac{1989}{2285}$ & $\begin{array}{l}1990 \\
2166\end{array}$ & $\frac{1991}{3128}$ & $\frac{1992}{2803}$ & $\begin{array}{l}1993 \\
3354\end{array}$ & $\begin{array}{l}1994 \\
3550\end{array}$ & $\begin{array}{l}1995 \\
3530\end{array}$ & $\begin{array}{l}1996 \\
3465\end{array}$ & $\begin{array}{l}1997 \\
3199\end{array}$ & $\frac{1998}{2847}$ & $\frac{1999}{2547}$ & $\frac{2000}{2863}$ & $\begin{array}{ll}2001 \\
2906\end{array}$ & $\begin{array}{ll}2002 \\
3257\end{array}$ & $\frac{2003}{3530}$ & $\begin{array}{l}2004 \\
3715\end{array}$ & $\begin{array}{ll}2005 \\
4027\end{array}$ & $\begin{array}{l}2006 \\
4811\end{array}$ & $\frac{2007}{3744}$ \\
\hline $\begin{array}{l}\text { Limites } \\
\text { Paitis }\end{array}$ & - & . & 10 & 10 & 10 & 10 & $10^{-}$ & $10^{-}$ & $10^{-}$ & $10^{-}$ & $100^{-1}+2$ & 3199 & $100^{-1}+2$ & 10 & 2000 & $\ln ^{-}$ & 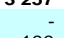 & $-0^{-}$ & - & $\cos ^{-}$ & - & $3 / 44$ \\
\hline $\begin{array}{l}\text { Tas de limites } \\
\text { Taux du paiement }\end{array}$ & 100 & 100 & $\begin{array}{l}100 \\
n a \\
n\end{array}$ & 100 & 100 & $\begin{array}{l}100 \\
n a\end{array}$ & $\begin{array}{l}100 \\
n a .\end{array}$ & $\begin{array}{l}100 \\
\text { na. }\end{array}$ & $\begin{array}{l}100 \\
n a .\end{array}$ & $\begin{array}{l}100 \\
\text { na. }\end{array}$ & $\begin{array}{l}100 \\
\text { na }\end{array}$ & 100 & 100 & 100 & 100 & 100 & 100 & 100 & 100 & 100 & 100 & 100 \\
\hline $\begin{array}{l}\text { Taur du paiement } \\
\text { Avec c. (volontaire) } \\
\text { Pas anplicable }\end{array}$ & 100 & 100 & 100 & 100 & 100 & 100 & 100 & 100 & 100 & 100 & 100 & 100 & 99 & 99 & 100 & 97 & 96 & $\begin{array}{l}\text { n.a. } \\
96 \\
96\end{array}$ & 95 & $\begin{array}{l}\text { n.a. } \\
96\end{array}$ & $\begin{array}{l}\text { n.a. } \\
97\end{array}$ & $\begin{array}{r}\text { n.a. } \\
96\end{array}$ \\
\hline $\begin{array}{l}\text { Pas applicable } \\
\text { Produit }\end{array}$ & n.a. & n.a. & n.a. & n.a. & n.a. & n.a. & n.a. & n.a. & n.a. & n.a. & n.a. & n.a. & n.a. & n.a. & n.a. & n.a. & n.a. & n.a. & n.a. & n.a. & n.a. & $\begin{array}{c}4 \\
\text { n.a. } \\
\text { nat }\end{array}$ \\
\hline $\begin{array}{l}\mathrm{S} / \mathrm{Na} / \mathrm{Rc} / \mathrm{Rv} \\
\text { Frontions }\end{array}$ & n.a. & n.a. & n.a. & n.a. & n.a. & n.a. & n.a. & n.a. & n.a. & n.a. & n.a. & n.a. & n.a. & n.a. & n.a. & n.a. & n.a. & n.a. & n.a. & n.a. & n.a. & n.a. \\
\hline $\begin{array}{l}\text { Exceptions } \\
\text { Australie (USD) }\end{array}$ & & & & & & & n.a. & n.... & n.a. & n.a. & n.a. & n.a. & n.a. & & n.a. & n.a. & n.a. & n.a. & n.a. & n.a. & n.a. & \\
\hline Limites & & & & & & & & & & & & & & & & & & & & & & \\
\hline $\begin{array}{l}\text { as de limites } \\
\text { Taux du paiement }\end{array}$ & n.a. & n.a. & n.a. & n.a. & n.a. & n.a. & $\begin{array}{l}100 \\
\text { n.a. }\end{array}$ & $\begin{array}{l}100 \\
\text { n.a. }\end{array}$ & $\begin{array}{l}100 \\
\text { na. }\end{array}$ & $\begin{array}{l}100 \\
\text { n.a. }\end{array}$ & $\begin{array}{l}100 \\
\text { n.a. }\end{array}$ & $\begin{array}{l}100 \\
\text { n.a. }\end{array}$ & $\begin{array}{l}100 \\
\text { n.a. }\end{array}$ & n.a. & n.a. & n.a. & n.a. & n.a. & n.a. & n.a. & n.a. & n.a. \\
\hline $\begin{array}{l}\text { Avec c. (volontaire) } \\
\text { Pand }\end{array}$ & & n.a. & n.a. & & & & n.a. & . & . & m.a. & & & m.a. & & & & & & & & & \\
\hline $\begin{array}{l}\text { Pas applicable } \\
\text { Produit }\end{array}$ & n.a. & n.a. & n.a. & n.a. & n.a. & n.a. & $\begin{array}{l}100 \\
\text { n.a. }\end{array}$ & $\begin{array}{l}100 \\
\text { n.a. }\end{array}$ & $\begin{array}{l}100 \\
\text { n.a. }\end{array}$ & $\begin{array}{l}100 \\
\text { n.a. }\end{array}$ & $\begin{array}{l}100 \\
\text { n.a. }\end{array}$ & $\begin{array}{l}100 \\
\text { n.a. } \\
\text { no. }\end{array}$ & $\begin{array}{l}100 \\
\text { n.a. }\end{array}$ & n.a. & n.a. & n.a. & n.a. & n.a. & n.a. & n.a. & n.a. & n.a. \\
\hline $\mathrm{S} / \mathrm{Na} / \mathrm{Rc} / \mathrm{Rv}$ & n.a. & n.a. & n.a. & n.a. & n.a. & n.a. & n.a. & n.a. & n.a. & n.a. & n.a. & n.a. & n.a. & n.a. & n.a. & n.a. & n.a. & n.a. & n.a. & n.a. & n.a. & n.a. \\
\hline $\begin{array}{l}\text { Exceptions } \\
\text { Canada (USD) }\end{array}$ & n.a. & n.a. & n.a. & n.a. & n.a. & n.a. & n.a. & n.a. & n.a. & n.a. & n.a. & n.a. & n.a. & n.a. & n.a. & n.a. & n.a. & n.a. & $\begin{array}{l}\text { n.a. } \\
30\end{array}$ & n.a. & n.a. & n.a. \\
\hline Limites & & & & & & & & & & & & & & & & & & & & & & \\
\hline $\begin{array}{l}\text { Pas de limites } \\
\text { Tauxdupiement }\end{array}$ & 100 & 100 & 100 & 100 & 100 & 100 & 100 & 100 & & & & 100 & 100 & & & & & & 100 & 100 & 100 & \\
\hline $\begin{array}{l}\text { Taux du paiement } \\
\text { Avec c. (volontaire) }\end{array}$ & $\begin{array}{l}\text { n.a. } \\
100\end{array}$ & $\begin{array}{l}\text { n.a. } \\
100\end{array}$ & $\begin{array}{l}\text { n.a. } \\
100\end{array}$ & $\begin{array}{l}\text { n.a. } \\
100\end{array}$ & $\begin{array}{l}\text { n.a. } \\
100\end{array}$ & $\begin{array}{l}\text { n.a. } \\
100\end{array}$ & $\begin{array}{l}\text { n.a. } \\
100\end{array}$ & $\begin{array}{l}\text { n.a. } \\
100\end{array}$ & n.a. & n.a. & n.a. & $\begin{array}{l}\text { n.a. } \\
100\end{array}$ & $\begin{array}{l}\text { n.a. } \\
100\end{array}$ & n.a. & n.a. & n.a. & n.a. & n.a. & $\begin{array}{l}\text { n.a. } \\
100\end{array}$ & $\begin{array}{l}\text { n.a. } \\
100\end{array}$ & $\begin{array}{l}\text { n.a. } \\
100\end{array}$ & n.a. \\
\hline $\begin{array}{l}\text { Pas applicable } \\
\text { Produit }\end{array}$ & n.a. & п.a. & n.a. & n.a. & n.a. & n.a. & n.a. & n.a. & n.a. & n.a. & n.a. & n.a. & n.a. & n.a. & n.a. & n.a. & n.a. & n.a. & n.a. & n.a. & n.a. & n.a. \\
\hline S/Na/Rc/Rv & n.a. & n.a. & n.a. & n.a. & n.a. & n.a. & n.a. & n.a. & n.a. & n.a. & n.a. & n.a. & n.a. & n.a. & n.a. & n.a. & n.a. & n.a. & n.a. & n.a. & n.a. & n.a. \\
\hline $\begin{array}{l}\text { Exceptions } \\
\text { UE (USD) }\end{array}$ & $\frac{\text { n.a. }}{289}$ & $\frac{\text { n.a. }}{221}$ & $\begin{array}{l}\text { n.a. } \\
494\end{array}$ & $\begin{array}{l}\text { n.a. } \\
870\end{array}$ & $\frac{\text { n.a. }}{600}$ & $\begin{array}{l}\text { n.a. } \\
1465\end{array}$ & $\begin{array}{l}\text { n.a. } \\
1107\end{array}$ & $\begin{array}{l}\text { n.a. } \\
1743\end{array}$ & $\frac{\text { n.a. }}{1664}$ & $\frac{\text { n.a. }}{1661}$ & $\frac{\text { n.a. }}{1612}$ & $\begin{aligned} \text { n.a. } \\
1341\end{aligned}$ & $\begin{array}{l}\text { n.a. } \\
7766\end{array}$ & $\frac{\text { n.a. }}{860}$ & $\begin{array}{l}\text { n.a. } \\
1104\end{array}$ & $\frac{\text { n.a. }}{1000}$ & $\frac{\text { n.a. }}{1100}$ & $\begin{array}{l}\text { n.a. } \\
1274\end{array}$ & $\begin{array}{l}\text { n.a. } \\
1429\end{array}$ & $\frac{\text { n.a. }}{1606}$ & $\frac{\text { n.a. }}{2286}$ & $\frac{\text { n.a. }}{1390}$ \\
\hline $\begin{array}{l}\text { Limites } \\
\text { Pas de limites }\end{array}$ & 100 & 100 & 100 & 100 & 100 & 100 & 100 & 100 & 100 & 100 & 100 & 100 & 100 & 100 & 100 & 100 & 100 & 100 & 100 & 100 & 100 & 100 \\
\hline Taux du paiement & n.a. & n.a. & n.a. & n.a. & п.a. & n.a. & n.a. & n.a. & n.a. & n.a. & n.a. & n.a. & n.a. & n.a. & n.a. & n.a. & n.a. & n.a. & n.a. & n.a. & n.a. & n.a. \\
\hline $\begin{array}{l}\text { Avec c. (volontaire) } \\
\text { Pros applicable }\end{array}$ & 100 & 100 & 100 & 100 & 100 & 100 & 100 & 100 & 100 & 100 & 100 & 100 & 100 & 100 & 100 & $\begin{array}{r}93 \\
7\end{array}$ & $\begin{array}{l}90 \\
10\end{array}$ & $\begin{array}{r}91 \\
9\end{array}$ & $\begin{array}{r}91 \\
9\end{array}$ & $\begin{array}{r}92 \\
8\end{array}$ & $\begin{array}{r}96 \\
4\end{array}$ & $\begin{array}{r}93 \\
7\end{array}$ \\
\hline Produi & n.a. & n.a. & n.a. & n.a. & n.a. & n.a. & n.a. & n.a. & n.a. & n.a. & n.a. & n.a. & n.a. & n.a. & n.a. & n.a. & n.a. & n.a. & n.a. & n.a. & n.a. & n.a. \\
\hline $\begin{array}{l}\text { S/Na/Rc/Rv } \\
\text { Exceptions }\end{array}$ & $\begin{array}{l}\text { n.a. } \\
\text { n.a. }\end{array}$ & $\begin{array}{l}\text { n.a. } \\
\text { n.a. }\end{array}$ & $\begin{array}{l}\text { n.a. } \\
\text { n.a. }\end{array}$ & $\begin{array}{l}\text { n.a. } \\
\text { n.a. }\end{array}$ & $\begin{array}{l}\text { n.a. } \\
\text { n.a. }\end{array}$ & $\begin{array}{l}\text { n.a. } \\
\text { n.a. }\end{array}$ & $\begin{array}{l}\text { n.a. } \\
\text { n.a. } \\
\text { na }\end{array}$ & $\begin{array}{l}\text { n.a. } \\
\text { n.a. } \\
\text { na }\end{array}$ & $\begin{array}{l}\text { n.a. } \\
\text { n.a. }\end{array}$ & $\begin{array}{l}\text { n.a. } \\
\text { n.a. } \\
\text { nat }\end{array}$ & $\begin{array}{l}\text { n.a. } \\
\text { n.a. }\end{array}$ & $\begin{array}{l}\text { n.a. } \\
\text { n.a. } \\
\text { na }\end{array}$ & $\begin{array}{l}\text { n.a. } \\
\text { n.a. }\end{array}$ & $\begin{array}{l}\text { n.a. } \\
\text { n.a. }\end{array}$ & $\begin{array}{l}\text { n.a. } \\
\text { n.a. }\end{array}$ & $\begin{array}{l}\text { n.a. } \\
\text { n.a. }\end{array}$ & $\begin{array}{l}\text { n.a. } \\
\text { n.a. }\end{array}$ & $\begin{array}{l}\text { n.a. } \\
\text { n.a. }\end{array}$ & $\begin{array}{l}\text { n.a. } \\
\text { n.a. }\end{array}$ & $\begin{array}{l}\text { n.a. } \\
\text { n.a. }\end{array}$ & $\begin{array}{l}\text { n.a. } \\
\text { n.a. }\end{array}$ & $\begin{array}{l}\text { n.a. } \\
\text { n.a. }\end{array}$ \\
\hline Islande (USD) & & & & & & & & & & & & & & & & & 0 & 2 & 1 & 0 & 1 & \\
\hline $\begin{array}{l}\text { Limites } \\
\text { Pas de limites }\end{array}$ & & & & & & & & & & & & & & & & & 100 & 100 & 100 & & & \\
\hline $\begin{array}{l}\text { Taux du paiement } \\
\text { Avec c. (volontaire) } \\
\text { Pas applicable }\end{array}$ & n.a. & n.a. & n.a. & n.a. & n.a. & n.a. & п.a. & n.a. & n.a. & n.a. & n.a. & n.a. & n.a. & n.a. & n.a. & n.a. & $\begin{array}{l}\text { n.a. } \\
100\end{array}$ & $\begin{array}{l}\text { n.a. } \\
100\end{array}$ & $\begin{array}{l}\text { n.a. } \\
100\end{array}$ & $\begin{array}{l}\text { n.a. } \\
100\end{array}$ & $\begin{array}{l}\text { n.a. } \\
100\end{array}$ & n.a. \\
\hline $\begin{array}{l}\text { Produit } \\
\text { Prot }\end{array}$ & n.a. & n.a. & n.a. & n.a. & n.a. & n.a. & n.a. & n.a. & n.a. & n.a. & n.a. & n.a. & n.a. & n.a. & n.a. & n.a. & n.a. & n.a. & n.a. & n.a. & n.a. & n.a. \\
\hline & $\begin{array}{l}\text { n.a. } \\
\text { n.a. }\end{array}$ & $\begin{array}{l}\text { n.a. } \\
\text { n.a. }\end{array}$ & $\begin{array}{l}\text { n.a. } \\
\text { n.a. }\end{array}$ & $\begin{array}{l}\text { n.a. } \\
\text { n.a. }\end{array}$ & $\begin{array}{l}\text { n.a. } \\
\text { n.a. } \\
\text { na }\end{array}$ & $\begin{array}{l}\text { n.a. } \\
\text { n.a. } \\
\text { na }\end{array}$ & $\begin{array}{l}\text { n.a. } \\
\text { n.a. }\end{array}$ & $\begin{array}{l}\text { n.a. } \\
\text { n.a. }\end{array}$ & $\begin{array}{l}\text { n.a. } \\
\text { n.a. } \\
\text { na }\end{array}$ & $\begin{array}{l}\text { n.a. } \\
\text { n.a. } \\
\text { na }\end{array}$ & n.a. & $\begin{array}{l}\text { n.a. } \\
\text { n.a. }\end{array}$ & n.a. & $\begin{array}{l}\text { n.a. } \\
\text { n.a. }\end{array}$ & $\begin{array}{l}\text { n.a. } \\
\text { n.a. }\end{array}$ & $\begin{array}{l}\text { n.a. } \\
\text { n.a. }\end{array}$ & $\begin{array}{l}\text { n.a. } \\
\text { n.a. }\end{array}$ & n.a. & $\begin{array}{l}\text { n.a. } \\
\text { n.a. }\end{array}$ & $\begin{array}{l}\text { n.a. } \\
\text { n.a. }\end{array}$ & $\begin{array}{l}\text { n.a. } \\
\text { n.a. }\end{array}$ & $\begin{array}{l}\text { n.a. } \\
\text { n.a. }\end{array}$ \\
\hline $\begin{array}{l}\text { Japon (USD) } \\
\text { Limites }\end{array}$ & & & & & & & & & & & & & & & & & & & & & & \\
\hline $\begin{array}{l}\text { Pas de limites } \\
\text { Taud du paiement }\end{array}$ & n.a. & n.a. & n.a. & n.a. & n.a. & n.a. & & n.a. & n.a. & n.a. & n.a. & n.a. & n.a. & n.a. & n.a. & n.a. & n.a. & п.a. & n.a. & n.a. & n.a. & n.a. \\
\hline $\begin{array}{l}\text { Taux du paiement } \\
\text { Avec c. (volontaire) } \\
\text { Pas applicable }\end{array}$ & . & m.a. & n.a. & & & n.a. & n.a. & & & & n.a. & & n.a. & & & n.a. & & & & & & \\
\hline & n.a. & n.a. & n.a. & n.a. & n.a. & n.a. & n.a. & n.a. & n.a. & n.a. & n.a. & n.a. & п.a. & n.a. & n.a. & n.a. & п.a. & п.a. & п.a. & n.a. & n.a. & n.a. \\
\hline & n.a. & n.a. & n.a. & n.a. & n.a. & n.a. & n.a. & n.a. & n.a. & n.a. & n.a. & n.a. & n.a. & n.a. & n.a. & n.a. & n.a. & n.a. & n.a. & n.a. & n.a. & n.a. \\
\hline $\begin{array}{l}\text { Exceptions } \\
\text { Corée (USD) }\end{array}$ & n.a. & n.a. & n.a. & n.a. & n.a. & n.a. 4 & n.a. & n.a. 1 & n.a. & n.a. & n.a. & n.a. 1 & n.a. & n.a. & n.a. & n.a. & n.a. & n.a. & $\frac{\text { n.a. }}{14}$ & n.e. & & \\
\hline $\begin{array}{l}\text { Limites } \\
\text { Pas de lim }\end{array}$ & & & & & & $100^{\circ}-(x)$ & 100 & 100 & & & & 100 & 100 & & 100 & & 100 & 0 & 100 & $100^{\circ}$ & & \\
\hline $\begin{array}{l}\text { Taux du paiement } \\
\text { Avec c. (volontaire) }\end{array}$ & n.a. & n.a. & n.a. & n.a. & $\begin{array}{l}\text { n.a. } \\
100\end{array}$ & $\begin{array}{l}\text { n.a. } \\
100\end{array}$ & $\begin{array}{l}\text { n.a. } \\
100\end{array}$ & $\begin{array}{l}\text { n.a. } \\
100\end{array}$ & n.a. & n.a. & n.a. & $\begin{array}{l}\text { n.a. } \\
100\end{array}$ & $\begin{array}{l}\text { n.a. } \\
100\end{array}$ & $\begin{array}{l}\text { n.a. } \\
\text { noo }\end{array}$ & $\begin{array}{l}\text { n.a. } \\
100\end{array}$ & $\begin{array}{l}\text { n.a. } \\
100\end{array}$ & $\begin{array}{l}\text { n.a. } \\
100\end{array}$ & $\begin{array}{l}\text { n.a. } \\
\text { noo. }\end{array}$ & $\begin{array}{l}\text { n.a. } \\
\text { noo. }\end{array}$ & $\begin{array}{l}\text { n.a. } \\
100\end{array}$ & n.a. & n.a. \\
\hline & & & & n.a & n.a. & n.a. & n.a. & n.a. & & & n.a. & n.a. & n.a. & n.a. & n.a. & n.a. & n.a. & n.a. & n.a. & n.a. & n.a. & n.a. \\
\hline $\mathrm{S} / \mathrm{Na} / \mathrm{Rc} / \mathrm{Rv}$ & n. 2 & n.a & n.a & & n.a. & n.a. & & n.a. & & & n.a. & n.a. & n.a. & n.a. & n.a. & n.a. & n.a. & n.a. & n.a. & n.a. & n.a. & n.a. \\
\hline Exceptions & n.a. & n.a. & n.a. & n.a. & n.a. & n.a. & n.a. & n.a. & n.a. & n.a. & n.a. & n.a. & n.a. & n.a. & n.a. & n.a. & n.a. & n.a. & n.a. & n.a. & n.a. & \\
\hline
\end{tabular}

ANALYSE DE LA COMPOSITION DU SOUTIEN AUX PRODUCTEURS : NOUVEAUX OUTILS ET MÉTHODES @ OECD 2010 
Tableau annexe 9. Catégorie F (PN) : composition de la catégorie par pays et étiquette, 1986-2007 (suite)

\section{Part de la catégorie en pourcentage}

\begin{tabular}{|c|c|c|c|c|c|c|c|c|c|c|c|c|c|c|c|c|c|c|c|c|c|c|}
\hline & 1986 & 1987 & 1988 & 1989 & 1990 & 1991 & 1992 & 1993 & 1994 & 1995 & 1996 & 1997 & 1998 & 1999 & 2000 & 2001 & 2002 & 2003 & 2004 & 2005 & 2006 & 2007 \\
\hline Mexique (USD) & - & - & - & & - & & - & - & - & - & - & - & - & - & - & - & - & 43 & 20 & - & 13 & \\
\hline $\begin{array}{l}\text { Limites } \\
\text { Pas de limites }\end{array}$ & & & & & & & & & & & & & & & & & & 100 & 100 & & 100 & \\
\hline $\begin{array}{l}\text { Taux du paiement } \\
\text { Avec c. (volontaire) } \\
\text { Pas anplicable }\end{array}$ & n.a. & n.a. & n.a. & n.a. & n.a. & n.a. & n.a. & n.a. & n.a. & n.a. & n.a. & n.a. & n.a. & n.a. & n.a. & n.a. & n.a. & $\begin{array}{l}\text { n.a. } \\
100\end{array}$ & $\begin{array}{l}\text { n.a. } \\
100\end{array}$ & n.a. & $\begin{array}{l}\text { n.a. } \\
100\end{array}$ & n.a. \\
\hline $\begin{array}{l}\text { Pas applicable } \\
\text { Produit }\end{array}$ & n.a. & n.a. & n.a. & n.a. & n.a. & n.a. & n.a. & n.a. & n.a. & n.a. & n.a. & n.a. & n.a. & n.a. & n.a. & n.a. & n.a. & n.a. & n.a. & n.a. & n.a. & n.a. \\
\hline $\mathrm{S} / \mathrm{Na} / \mathrm{Rc} / \mathrm{Rv}$ & n.a. & n.a. & n.a. & n.a. & n.a. & n.a. & n.a. & n.a. & n.a. & n.a. & n.a. & n.a. & n.a. & n.a. & n.a. & n.a. & n.a. & n.a. & n.a. & n.a. & n.a. & n.a. \\
\hline \multirow{2}{*}{\multicolumn{23}{|c|}{$\begin{array}{l}\text { Nelle-Zélande (USD) } \\
\text { Limites }\end{array}$}} \\
\hline & & & & & & & & & & & & & & & & & & & & & & \\
\hline $\begin{array}{l}\text { Taux du paiement } \\
\text { Avec c. (volontaire) } \\
\text { Pas apolicable }\end{array}$ & n.a. & n.a. & n.a. & n.a. & n.a. & n.a. & n.a. & n.a. & n.a. & n.a. & n.a. & n.a. & n.a. & n.a. & n.a. & n.a. & n.a. & n.a. & n.a. & n.a. & n.a. & n.a. \\
\hline $\begin{array}{l}\text { Pas appilcabole } \\
\text { Produit }\end{array}$ & n.a. & n.a. & n.a. & n.a. & n.a. & n.a. & n.a. & n.a. & n.a. & n.a. & n.a. & n.a. & n.a. & n.a. & n.a. & n.a. & n.a. & n.a. & n.a. & n.a. & n.a. & n.a. \\
\hline $\mathrm{S} / \mathrm{Na} / \mathrm{Rc} / \mathrm{Rv}$ & n.a. & n.a. & n.a. & n.a. & n.a. & n.a. & n.a. & n.a. & n.a. & n.a. & n.a. & n.a. & n.a. & n.a. & n.a. & n.a. & n.a. & n.a. & n.a. & n.a. & n.a. & $\begin{array}{l}\text { n.a. } \\
\text { n. }\end{array}$ \\
\hline \multirow{2}{*}{\multicolumn{23}{|c|}{$\begin{array}{l}\text { Exceptions } \\
\begin{array}{c}\text { Norvège (USD) } \\
\text { Limites }\end{array}\end{array}$}} \\
\hline & & & & & & & & & & & & & & & & & & & & & & \\
\hline & & & & & 100 & 100 & 100 & 100 & 100 & 100 & 100 & 100 & & & & & & & & & & \\
\hline $\begin{array}{l}\text { Taux du paiement } \\
\text { Avec c. (volontaire) }\end{array}$ & n.a. & n.a. & n.a. & n.a. & n.a. & n.a. & n.a. & n.a. & n.a. & n.a. & n.a. & n.a. & n.a. & n.a. & n.a. & n.a. & n.a. & n.a. & n.a. & n.a. & n.a. & n.a. \\
\hline Pas applicable & & & & & 100 & 100 & 100 & 100 & 100 & 100 & 100 & 100 & & & & & & & & & & \\
\hline Produit & n.a. & n.a. & n.a. & n.a. & n.a. & n.a. & n.a. & n.a. & n.a. & n.a. & n.a. & n.a. & n.a. & n.a. & n.a. & n.a. & n.a. & n.a. & n.a. & n.a. & n.a. & n.a. \\
\hline $\mathrm{S} / \mathrm{Na} / \mathrm{Rc} / \mathrm{Rv}$ & n.a. & n.a. & n.a. & n.a. & n.a. & n.a. & n.a. & n.a. & n.a. & n.a. & n.a. & n.a. & n.a. & n.a. & n.a. & n.a. & n.a. & n.a. & n.a. & n.a. & n.a. & n.a. \\
\hline $\begin{array}{l}\text { Exceptions } \\
\text { Suisse (USD) }\end{array}$ & n.a. & n.a. & n.a. & n.a. & n.a. & n.a. & n.a. & $\frac{\text { n.a. }}{20}$ & $\frac{\text { n.a. }}{26}$ & n.a. & $\frac{\text { n.a. }}{50}$ & n.a. & n.a. & $\frac{\text { n.a. }}{51}$ & $\frac{\text { n.a. }}{50}$ & $\frac{n . a .}{58}$ & n.a. & $\frac{\text { n.a. }}{85}$ & $\frac{\text { n.a. }}{100}$ & $\begin{array}{l}\text { n.a. } \\
105\end{array}$ & $\begin{array}{l}\text { n.a. } \\
106\end{array}$ & $\frac{\text { n.a. }}{115}$ \\
\hline Limites & & & & & & & & +0 & & & & & & & & & & & & & & \\
\hline $\begin{array}{l}\text { Pas de limites } \\
\text { Taux du paiement }\end{array}$ & $n$ & $n$ & $n$ & na & n & na & na & 100 & 100 & 100 & 100 & 100 & 100 & 100 & 100 & 100 & 100 & 100 & 100 & 100 & 100 & 100 \\
\hline 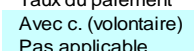 & n.d. & n... & n.a. & n.d. & ก.d. & ก.d. & I. & 100 & 100 & 100 & 100 & 100 & 100 & 100 & 100 & 100 & 100 & 100 & 100 & 100 & 100 & 100 \\
\hline $\begin{array}{l}\text { Pas applicable } \\
\text { Produit }\end{array}$ & n.a. & n.a. & n.a. & n.a. & n.a. & n.a. & n.a. & n.a. & n.a. & n.a. & n.a. & n.a. & n.a. & n.a. & n.a. & n.a. & n.a. & n.a. & n.a. & n.a. & n.a. & n.a. \\
\hline $\mathrm{S} / \mathrm{Na} / \mathrm{Rc} / \mathrm{Rv}$ & n.a. & n.a. & n.a. & n.a. & n.a. & n.a. & n.a. & n.a. & n.a. & n.a. & n.a. & n.a. & n.a. & n.a. & п.а. & n.a. & n.a. & п.а. & n.a. & n.a. & n.a. & n.a. \\
\hline \multirow{2}{*}{\multicolumn{23}{|c|}{$\begin{array}{l}\text { Turquie (USD) } \\
\text { Limites }\end{array}$}} \\
\hline & & & & & & & & & & & & & & & & & & & & & & \\
\hline $\begin{array}{l}\text { Pas de limites } \\
\text { Taux du paiement }\end{array}$ & п.а. & п.а. & п.a. & n.a. & n.a. & n.a. & n.a. & n.a. & n.a. & n.a. & n.a. & n.a. & n.a. & n.a. & n.a. & n.a. & n.a. & n.a. & n.a. & п.а. & n.a. & n.a. \\
\hline \multicolumn{23}{|l|}{$\begin{array}{l}\text { Avec c. (volontaire) } \\
\text { Pas applicable }\end{array}$} \\
\hline $\begin{array}{l}\text { Produit } \\
\text { Procale }\end{array}$ & n.a. & n.a. & n.a. & n.a. & n.a. & n.a. & n.a. & n.a. & n.a. & n.a. & n.a. & n.a. & n.a. & n.a. & n.a. & n.a. & n.a. & n.a. & n.a. & n.a. & n.a. & n.a. \\
\hline $\begin{array}{l}\mathrm{S} / \mathrm{Na} / \mathrm{Rc} / \mathrm{Rv} \\
\text { Excentions }\end{array}$ & n.a. & n.a. & n.a. & n.a. & n.a. & n.a. & n.a. & n.a. & n.a. & n.a. & n.a. & n.a. & n.a. & n.a. & n.a. & n.a. & n.a. & n.a. & n.a. & n.a. & n.a. & n.a. \\
\hline $\begin{array}{l}\text { Exceptitions } \\
\text { Etats-Unis (USD) }\end{array}$ & n.a. & $\frac{\text { n.a. }}{686}$ & $\begin{array}{c}\text { n.a. } \\
1059\end{array}$ & $\frac{\text { n.a. }}{1381}$ & $\begin{array}{l}\text { n.a. } \\
1544\end{array}$ & $\begin{array}{c}\text { n.a. } \\
1651\end{array}$ & $\begin{array}{r}\text { n.a. } \\
1681\end{array}$ & $\begin{array}{r}\text { n.a. } \\
1599\end{array}$ & $\begin{array}{r}\text { n.a. } \\
1854\end{array}$ & $\begin{array}{r}\text { n.a. } \\
1819\end{array}$ & $\begin{array}{r}\text { n.a. } \\
1791\end{array}$ & $\begin{array}{r}\text { n.a. } \\
1809\end{array}$ & $\begin{array}{r}\text { n.a. } \\
2017\end{array}$ & $\begin{array}{r}\text { n.a. } \\
1636\end{array}$ & $\begin{array}{r}\text { n.a. } \\
1707\end{array}$ & $\begin{array}{r}\text { n.a. } \\
1844\end{array}$ & $\begin{array}{c}\text { n.a. } \\
2084\end{array}$ & n.a. & $\begin{array}{l}\text { n.a. } \\
2120\end{array}$ & $\begin{array}{l}\text { n.a. } \\
2253\end{array}$ & $\begin{array}{l}\text { n.a. } \\
2400\end{array}$ & n.a. \\
\hline $\begin{array}{l}\text { Limites } \\
\text { (ind) }\end{array}$ & & & & & & & & & & & & & & & & & & & & & 2402 & \\
\hline de limite & 100 & 100 & 100 & 100 & 100 & 100 & 100 & 100 & 100 & 100 & 100 & 100 & 100 & 100 & 100 & 100 & 100 & 100 & 100 & 100 & 100 & 100 \\
\hline & n.a. & n.a. & n.a. & n.a. & n.a. & n.a. & n.a. & n.a. & & n.a. & & n.a. & n.a. & n.a. & n.a. & n.a. & n.a. & n.a. & n.a. & n.a. & n.a. & n.a. \\
\hline $\begin{array}{l}\text { Avec c. (volontaire) } \\
\text { Pas apolicable }\end{array}$ & 100 & 100 & 100 & 100 & 100 & 100 & 100 & 100 & 100 & 100 & 100 & 100 & $\begin{array}{r}99 \\
1\end{array}$ & $\begin{array}{r}99 \\
1\end{array}$ & 100 & $\begin{array}{r}99 \\
1\end{array}$ & $\begin{array}{r}99 \\
1\end{array}$ & $\begin{array}{r}99 \\
1\end{array}$ & $\begin{array}{r}98 \\
2\end{array}$ & $\begin{array}{r}98 \\
2\end{array}$ & $\begin{array}{r}98 \\
2\end{array}$ & $\begin{array}{r}98 \\
2\end{array}$ \\
\hline Produit & n.a. & n.a. & n.a. & n.a. & n.a. & n.a. & n.a. & n.a. & n.a. & n.a. & п.a. & n.a. & n.a. & n.a. & п.а. & n.a. & n.a. & n.a. & n.a. & n.a. & n.a. & n.a. \\
\hline & & & n.a. & & n.a. & & n.a. & & & & & & & & & & n.a. & n.a. & n.a. & n.a. & n.a. & \\
\hline Exceptions & n.a. & n.a. & n.a. & n.a. & n.a. & n.a. & n.a. & n.a. & n.a. & n.a. & n.a. & n.a. & n.a. & n.a. & n.a. & n.a. & n.a. & n.a. & n.a. & n.a. & n.a. & n.a. \\
\hline
\end{tabular}


Tableau annexe 10. Australie : composition de l'ESP par catégorie et étiquette, 1986-2007

Part en pourcentage dans l'ESP

\begin{tabular}{|c|c|c|c|c|c|c|c|c|c|c|c|c|c|c|c|c|c|c|c|c|c|c|}
\hline & 1986 & 1987 & 1988 & 1989 & 1990 & 1991 & 1992 & 1993 & 1994 & 1995 & 1996 & 1997 & 1998 & 1999 & 2000 & 2001 & 2002 & 2003 & 2004 & 2005 & 2006 & 2007 \\
\hline PSE (millions AUD) & 1870 & 1512 & 938 & 1711 & 2077 & 1700 & 1488 & 2577 & 2237 & 1693 & 2754 & 1681 & 2052 & 1864 & 1564 & 1843 & 3330 & 1777 & 1560 & 1802 & 2000 & 2238 \\
\hline \multicolumn{23}{|c|}{ Composition par catégorie avec labels correspondants } \\
\hline Production & 61,3 & 48,7 & 24,4 & 61,4 & 73,2 & 56,1 & 34,6 & 62,5 & 53,2 & 36,2 & 59,3 & 44,1 & 54,5 & 47,5 & 0,5 & 7,8 & 42,4 & 0,2 & 0,1 & 4,9 & 0,1 & 0,0 \\
\hline Intrants & 28,9 & 32,6 & 44,5 & 26,5 & 26,2 & 40,9 & 56,7 & 30,0 & 35,2 & 46,7 & 30,4 & 45,7 & 37,1 & 44,6 & 72,1 & 54,3 & 27,3 & 52,5 & 63,3 & 49,2 & 59,5 & 44,5 \\
\hline $\mathrm{S} / \mathrm{Na} / \mathrm{Rc} / \mathrm{Rv}$ & 9,8 & 18,7 & 31,1 & 12,1 & 0,6 & 3,0 & 8,6 & 7,3 & 11,4 & 17,0 & 10,4 & 10,2 & 8,2 & 7,9 & 27,4 & 37,9 & 30,3 & 47,3 & 36,5 & 45,9 & 40,4 & 55,5 \\
\hline Superficie & $\cdot$ & $\cdot$ & $\cdot$ & $\cdot$ & $\cdot$ & $\cdot$ & 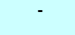 & $\cdot$ & - & $\cdot$ & $\cdot$ & $\cdot$ & - & $\cdot$ & $\cdot$ & - & - & $\cdot$ & $\cdot$ & $\cdot$ & - & - \\
\hline $\begin{array}{l}\text { Nore d'animaux } \\
\text { Recettes }\end{array}$ & - & - & - & - & - & - & & - & - & $\cdot$ & - & $\cdot$ & - & - & 10.4 & 11,9 & 6.7 & 12,3 & 14.6 & 12.8 & 11,3 & 11,0 \\
\hline Revenu & 9,8 & 18,7 & 31,1 & 12,1 & 0,6 & 3,0 & 8,6 & 7,3 & 11,4 & 17,0 & 10,4 & 10,2 & 8,2 & 7,9 & 17,0 & 26,0 & 23,6 & 34,9 & 21,9 & 33,1 & 29,1 & 44,5 \\
\hline Autres produits & & - & - & - & - & - & 0,1 & 0,2 & 0,2 & 0,1 & 0,0 & 0,0 & 0,3 & - & - & - & - & - & - & - & - & \\
\hline Courant & 90,4 & 81,5 & 69,1 & 88,0 & 99,5 & 97,2 & 91,7 & 92,8 & 88,9 & 83,3 & 90,9 & 90,2 & 92,3 & 92,6 & 73,1 & 62,5 & 69,9 & 54,5 & 64,2 & 56,4 & 61,5 & 47,1 \\
\hline Non-courant & 9,6 & 18,5 & 30,9 & 12,0 & 0,5 & 2,8 & 8,3 & 7,2 & 11,1 & 16,7 & 9,1 & 9,8 & 7,7 & 7,4 & 26,9 & 37,5 & 30,1 & 45,5 & 35,8 & 43,6 & 38,5 & 52,9 \\
\hline Courant/variable & 80,9 & 69,6 & 50,8 & 78,5 & 90,4 & 84,4 & 74,9 & 83,7 & 77,2 & 67,4 & 81,9 & 78,1 & 81,8 & 81,8 & 58,7 & 52,0 & 64,8 & 41,5 & 50,6 & 40,2 & 45,1 & 31,6 \\
\hline Courantffixe & 9,4 & 11,8 & 18,3 & 9,5 & 9,1 & 12,8 & 16,8 & 9,0 & 11,5 & 15,9 & 8,9 & 12,1 & 10,2 & 10,8 & 14,4 & 10,5 & 5,2 & 13,0 & 13,6 & 16,2 & 16,4 & 15,5 \\
\hline Non-courant/variable & 9,6 & 18,5 & 30,9 & 12,0 & 0,5 & 0,4 & 5,4 & 4,0 & 4,8 & 8,4 & 5,5 & 6,9 & 4,8 & 5,2 & 24,7 & 35,2 & 25,9 & 37,7 & 27,5 & 28,7 & 21,8 & 20,3 \\
\hline Non-courantffixe & 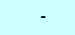 & - & 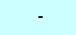 & - & - & 2,4 & 2,8 & 3,1 & 6,3 & 8,3 & 3,6 & 2,9 & 2,9 & 2,2 & 2,2 & 2,3 & 4,1 & 7,8 & 8,3 & 14,9 & 16,7 & 32,5 \\
\hline Autres produits & & & & & & & 0,1 & 0,2 & 0,2 & 0,1 & 0,0 & 0,0 & 0,3 & & & & & & & & & \\
\hline Production requise & 90,4 & 81,5 & 69,1 & 88,0 & 99,5 & 97,2 & 91,6 & 92,7 & 88,7 & 83,3 & 90,9 & 90,2 & 92,0 & 92,6 & 73,1 & 62,5 & 69,9 & 54,7 & 65,1 & 56,6 & 61,5 & 47,1 \\
\hline Un seul produit & 61,3 & 48,7 & 24,4 & 61,5 & 75,5 & 61,6 & 40,1 & 65,1 & 54,6 & 38,8 & 60,8 & 46,0 & 55,7 & 47,5 & 1,4 & 8,3 & 42,5 & 0,4 & 1,1 & 5,7 & 0,3 & 0,2 \\
\hline Un groupe de produits & 0,9 & 0,9 & 1,1 & 0,6 & 0,5 & 0,5 & 0,6 & 0,2 & 0,2 & 0,3 & 0,2 & 0,5 & 0,3 & 1,1 & 19,8 & 11,5 & 3,1 & 7,1 & 12,1 & 7,4 & 6,3 & 5,5 \\
\hline Tous les prod & 28,2 & 31,9 & 43,6 & 25,9 & 23,4 & 35,1 & 51,0 & 27,3 & 34,0 & 44,2 & 29,9 & 43,7 & 36,0 & 44,0 & 51,9 & 42,7 & 24,3 & 47,3 & 51,9 & 43,5 & 54,9 & 41,4 \\
\hline Production facultative & 9,6 & 18,5 & 30,9 & 12,0 & 0,5 & 2,8 & 8,4 & 7,3 & 11,3 & 16,7 & 9,1 & 9,8 & 8,0 & 7,4 & 26,9 & 37,5 & 30,1 & 45,3 & 34,9 & 43,4 & 38,5 & 52,9 \\
\hline \multicolumn{23}{|c|}{ Proportion de chaque label } \\
\hline Avec limites & & & & & & & & & & & & & & & 21,2 & 12,8 & 4,1 & 8,9 & 13,1 & 8,2 & 7,1 & 6,3 \\
\hline Sans limites & 100,0 & 100,0 & 100,0 & 100,0 & 100,0 & 100,0 & 100,0 & 100,0 & 100,0 & 100,0 & 100,0 & 100,0 & 100,0 & 100,0 & 78,8 & 87,2 & 95,9 & 91,1 & 86,9 & 91,8 & 92,9 & 93,7 \\
\hline Fixe & 9,4 & 11,8 & 18,3 & 9,5 & 9,1 & 15,2 & 19,6 & 12,1 & 17,8 & 24,2 & 12,5 & 15,0 & 13,2 & 13,0 & 16,6 & 12,8 & 9,3 & 20,8 & 21,9 & 31,1 & 33,1 & 48,0 \\
\hline Variable & 90,6 & 88,2 & 81,7 & 90,5 & 90,9 & 84,8 & 80,3 & 87,7 & 82,0 & 75,8 & 87,5 & 85,0 & 86,6 & 87,0 & 83,4 & 87,2 & 90,7 & 79,2 & 78,1 & 68,9 & 66,9 & 52,0 \\
\hline Avec c. (obligatoire) & & & & & & & & & & & & & & & 0,9 & 0,5 & 0,0 & 0,1 & & & & \\
\hline \\
\hline Sans contrainte & 38,7 & 51,3 & 75,6 & 38,6 & 26,8 & 43,9 & 65,3 & 37,4 & 46,6 & 63,8 & 40,7 & 55,8 & 45,3 & 52,5 & 98,6 & 91,7 & 57,6 & 99,7 & 99,9 & 95,1 & 99,9 & 100,0 \\
\hline Un seul produit & 61 & 48,7 & 24,4 & 61 & 75,5 & 61,6 & 40,1 & 65,1 & 54,6 & 38,8 & 60,8 & 46,0 & 55,7 & 47,5 & 1,4 & 8,3 & 42,5 & 0,4 & 1,1 & 5,7 & 0,3 & 0,2 \\
\hline Un groupe de produits & 0,9 & 0,9 & 1,1 & 0,6 & 0,5 & 0,5 & 0,6 & 0,2 & 0,2 & 0,3 & 0,2 & 0,5 & 0,3 & 1,1 & 19,8 & 11,5 & 3,1 & 7,1 & 12,1 & 7,4 & 6,3 & 5,5 \\
\hline Tous les produits & 28,2 & 31,9 & 43,6 & 25,9 & 23,4 & 35,1 & 51,0 & 27,3 & 34,0 & 44,2 & 29,9 & 43,7 & 36,0 & 44,0 & 51,9 & 42,7 & 24,3 & 47,3 & 51,9 & 43,5 & 54,9 & 41,4 \\
\hline & - & - & - & - & - & $\cdot$ & $\cdot$ & $\cdot$ & $\cdot$ & $\cdot$ & $\cdot$ & $\cdot$ & $\cdot$ & $\cdot$ & 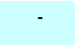 & - & - & 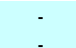 & $\therefore$ & $\therefore$ & - & \\
\hline Nbre d'animaux & - & - & - & - & - & - & & & - & - & 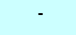 & - & - & - & & & & & & & & \\
\hline Recettes & - & - & - & - & - & - & - & - & - & - & $\therefore$ & - & - & - & 10,4 & 11,9 & 6,7 & 12,3 & 14,6 & 12,8 & 11,3 & 11,0 \\
\hline Revenu & 9,8 & 18,7 & 31,1 & 12,1 & 0,6 & 3,0 & 8,6 & 7,3 & 11,4 & 17,0 & 10,4 & 10,2 & 8,2 & 7,9 & 17,0 & 26,0 & 23,6 & 34,9 & 21,9 & 33,1 & 29,1 & 44,5 \\
\hline Avec exceptions & 9,6 & 18,5 & 30,9 & 12,0 & 0,5 & 2,8 & 8,3 & 7,2 & 11,1 & 16,7 & 9,1 & 9,8 & 7,7 & 6,2 & 23,7 & 29,4 & 17,7 & 31,5 & 28,8 & 37,0 & 33,0 & 47,9 \\
\hline Sans exceptions & & & & & & & & & & - & & & - & 1,2 & 3,2 & 8,1 & 12,3 & 13,8 & 6,1 & 6,4 & 5,5 & 4,9 \\
\hline
\end{tabular}

ANALYSE DE LA COMPOSITION DU SOUTIEN AUX PRODUCTEURS : NOUVEAUX OUTILS ET MÉTHODES ๑ OECD 2010 
Tableau annexe 11. Canada : composition de l'ESP par catégorie et étiquette, 1986-2007

Part en pourcentage de l'ESP

\begin{tabular}{|c|c|c|c|c|c|c|c|c|c|c|c|c|c|c|c|c|c|c|c|c|c|c|}
\hline & 1986 & 1987 & 1988 & 1989 & 1990 & 1991 & 1992 & 1993 & 1994 & 1995 & 1996 & 1997 & 1998 & 1999 & 2000 & 2001 & 2002 & 2003 & 2004 & 2005 & 2006 & 2007 \\
\hline$\overline{\text { PSE (millions CAD) }}$ & 8462 & 8784 & 6687 & 6052 & 7607 & 8297 & 6469 & 5670 & 5385 & 5684 & 4967 & 4356 & 5130 & 5473 & 6352 & 5753 & 8100 & 8522 & 7380 & 7873 & 8213 & 7521 \\
\hline \multicolumn{23}{|c|}{ Composition par catégorie avec labels correspondants } \\
\hline Production & & & & & 66,7 & & & & & & 47,1 & & 62,6 & 57,4 & & 44,2 & 50,3 & 48,0 & 43,6 & 45,2 & & \\
\hline Intrants & 13,9 & 13,6 & 27,6 & 22,0 & 15,9 & 13,8 & 13,9 & 15,9 & 14,9 & 13,5 & 13,3 & 15,1 & 9,9 & 9,3 & 8,6 & 8,8 & 5,8 & 5,9 & 7,7 & 7,5 & 8,2 & 8,5 \\
\hline $\mathrm{S} / \mathrm{Na} / \mathrm{Rc} / \mathrm{Rv}$ & 27,9 & 25,7 & 11,1 & 10,4 & 12,3 & 28,5 & 24,5 & 15,2 & 14,6 & 37,9 & 38,6 & 18,6 & 27,2 & 30,4 & 38,5 & 46,7 & 42,6 & 44,7 & 47,7 & 45,8 & 37,5 & 49,7 \\
\hline Superficie & 16,0 & 14,9 & 10,1 & 9,5 & 10,1 & 11,0 & 8,6 & 6,7 & 5,4 & 27,0 & 27,5 & 6,5 & 7,1 & 10,5 & 17,7 & 22,8 & 21,4 & 7,6 & 12,6 & 6,9 & 7,0 & 8,6 \\
\hline $\begin{array}{l}\text { Nore d'animaux } \\
\text { Recettes }\end{array}$ & $\begin{array}{r}0,6 \\
113\end{array}$ & $\begin{array}{r}0,5 \\
104\end{array}$ & $\begin{array}{l}0,5 \\
0,4\end{array}$ & $\begin{array}{l}0,7 \\
0,2\end{array}$ & $\begin{array}{l}0,7 \\
1,5\end{array}$ & $\begin{array}{r}0,6 \\
168\end{array}$ & $\begin{array}{r}0,9 \\
14,9\end{array}$ & $\begin{array}{l}1,1 \\
7,3\end{array}$ & 8,1 & $\begin{array}{l}1,1 \\
87\end{array}$ & $\begin{array}{l}1,6 \\
84\end{array}$ & $\begin{array}{l}1,8 \\
87\end{array}$ & $\begin{array}{l}1,6 \\
93\end{array}$ & $\begin{array}{l}1,6 \\
71\end{array}$ & $\begin{array}{r}1,4 \\
139\end{array}$ & $\begin{array}{r}1,1 \\
16\end{array}$ & $\begin{array}{l}1,1 \\
137\end{array}$ & $\begin{array}{r}2,5 \\
161\end{array}$ & 11,9 & 3,6 & 1,5 & $\begin{array}{r}2,0 \\
15,8\end{array}$ \\
\hline $\begin{array}{l}\text { Recettes } \\
\text { Revenu }\end{array}$ & 11,3 & 10,4 & & 0,2 & 1,5 & & & 7,3 & 8,1 & $\begin{array}{l}8,7 \\
1,1\end{array}$ & $\begin{array}{l}8,4 \\
1,2\end{array}$ & $\begin{array}{l}8,7 \\
1,5\end{array}$ & $\begin{array}{l}9,3 \\
9,2\end{array}$ & $\begin{array}{r}7,1 \\
11,2\end{array}$ & $\begin{array}{r}r 3,9 \\
5,4\end{array}$ & $\begin{array}{r}16,2 \\
6,5\end{array}$ & $\begin{array}{r}13,7 \\
6,4\end{array}$ & $\begin{array}{l}16,1 \\
18,5\end{array}$ & $\begin{array}{r}4,3 \\
18,9\end{array}$ & $\begin{array}{l}20,2 \\
15,1\end{array}$ & $\begin{array}{r}0,0 \\
29,0\end{array}$ & $\begin{array}{l}15,8 \\
23,2\end{array}$ \\
\hline Autres produits & 2,3 & 1,7 & 2,3 & 3,3 & 5,0 & 2,4 & 1,9 & 1,0 & 0,3 & 3,4 & $\begin{array}{l}1,0 \\
1,0\end{array}$ & 1,9 & 0,2 & $\begin{array}{r}2,2 \\
2,9\end{array}$ & 2,0 & 0,2 & $\begin{array}{l}1,4 \\
1,4\end{array}$ & 1,4 & 1,0 & 1,5 & & $\begin{array}{r}23,2 \\
0,5 \\
\end{array}$ \\
\hline Courant & 100,0 & 100,0 & 100,0 & 100,0 & 100,0 & 100,0 & 100,0 & 100,0 & 100,0 & 78,1 & 77,4 & 99,9 & 100,0 & 100,0 & 87,2 & 85,9 & 88,6 & 91,2 & 88,4 & 78,0 & 87,7 & $\begin{array}{ll}0,5 \\
77,6\end{array}$ \\
\hline Non-courant & & & & & & & & & & 21,9 & 22,6 & 0,1 & 0,0 & 0,0 & 12,8 & 14,1 & 11,4 & 8,8 & 11,6 & 22,0 & 12,3 & $\begin{array}{l}22,4 \\
22,0\end{array}$ \\
\hline Courant/variable & 33,7 & 30,3 & 20,4 & 21,0 & 11,0 & 33,7 & 29,7 & 19,3 & 13,1 & 11,7 & 12,4 & 13,1 & 20,3 & 25,0 & 19,9 & 24,1 & 24,9 & 35,2 & 33,1 & 23,3 & 25,2 & $\begin{array}{l}2,4 \\
10,3\end{array}$ \\
\hline Courantfixe & 64,1 & 68,0 & 77,3 & 75,7 & 84,0 & 63,9 & 68,4 & 79,7 & 86,6 & 63,0 & 64,0 & 84,9 & 79,5 & 72,1 & 65,4 & 61,5 & 62,3 & 54,5 & 54,2 & 53,2 & 62,1 & 66,8 \\
\hline Non-courant/variable & & & & & & & & & & 21,5 & 19,6 & 0,1 & & & & & & & & & 5,6 & \\
\hline Non-courantffixe & & & & & & & & & & 0,4 & 3,1 & 0,0 & 0,0 & 0,0 & 12,8 & 14,1 & 11,4 & 8,8 & 11,6 & 22,0 & 6,7 & 15,0 \\
\hline $\begin{array}{l}\text { Autres produits } \\
\text { Prnduction requis }\end{array}$ & 2,3 & $\begin{array}{l}1,7 \\
89\end{array}$ & 2,3 & 3,3 & $\begin{array}{r}5,0 \\
590\end{array}$ & 2,4 & $\begin{array}{r}1,9 \\
091\end{array}$ & 1,0 & 0,3 & $\begin{array}{r}3,4 \\
717 \\
717\end{array}$ & 1,0 & 1,9 & 0,2 & 2,9 & 2,0 & 0,2 & 1,4 & 1,4 & 1,0 & $\begin{array}{r}1,5 \\
-7,5\end{array}$ & 0,4 & $\begin{array}{r}0,5 \\
854\end{array}$ \\
\hline Production requise & $\begin{array}{l}97,7 \\
715\end{array}$ & $\begin{array}{l}98,3 \\
74,3\end{array}$ & 97,7 & 96,7 & $\begin{array}{l}95,0 \\
7717\end{array}$ & $\begin{array}{l}97,6 \\
586\end{array}$ & $\begin{array}{l}98,1 \\
692\end{array}$ & $\begin{array}{l}99,0 \\
757\end{array}$ & 99,7 & $\begin{array}{l}74,7 \\
516\end{array}$ & $\begin{array}{l}76,4 \\
550\end{array}$ & 98,0 & $\begin{array}{l}99,8 \\
713\end{array}$ & 97,1 & 85,3 & 85,7 & 87,2 & 89,8 & $\begin{array}{l}87,3 \\
598\end{array}$ & 76,5 & 88,9 & $\begin{array}{l}85,4 \\
51,5\end{array}$ \\
\hline Un seul produit & 71,5 & $\begin{array}{l}74,3 \\
110\end{array}$ & 68,1 & 74,4 & 71,7 & 58,6 & 69,2 & $\begin{array}{l}75,7 \\
0.2\end{array}$ & 76,7 & 51,6 & 55,0 & 72,7 & , & 69,4 & 64,4 & 61,3 & 68,1 & 然, & 然, & 33,3 & 60,5 & \\
\hline $\begin{array}{l}\text { Un groupe de produ } \\
\text { Tous les propdits }\end{array}$ & $\begin{array}{l}14,0 \\
122\end{array}$ & $\begin{array}{l}12,0 \\
120\end{array}$ & $\begin{array}{l}13,6 \\
161\end{array}$ & 1,7 & 9,2 & $\begin{array}{l}26,9 \\
121\end{array}$ & $\begin{array}{l}17,1 \\
11.9\end{array}$ & $\begin{array}{r}9,3 \\
140\end{array}$ & $\begin{array}{r}9,8 \\
13,\end{array}$ & $\begin{array}{r}9,4 \\
137\end{array}$ & $\begin{array}{r}7,6 \\
138\end{array}$ & $\begin{array}{r}9,6 \\
158\end{array}$ & $\begin{array}{l}10,1 \\
183\end{array}$ & $\begin{array}{l}8,0 \\
197\end{array}$ & 7,8 & 9,7 & $\begin{array}{l}6,8 \\
.18 \\
1.8\end{array}$ & $\begin{array}{l}14,33 \\
197\end{array}$ & $\begin{array}{l}6,5 \\
0,5\end{array}$ & $\begin{array}{l}5,1 \\
1,1\end{array}$ & , & $\begin{array}{l}15,1 \\
18,8\end{array}$ \\
\hline $\begin{array}{l}\text { Tous les produlits } \\
\text { Production facultative }\end{array}$ & 2,3 & $\begin{array}{l}12,0 \\
1,7\end{array}$ & $\begin{array}{r}10,1 \\
2,3\end{array}$ & $\begin{array}{r}20,6 \\
3,3\end{array}$ & $\begin{array}{r}4,0 \\
5,0\end{array}$ & 2,4 & 11,9 & $\begin{array}{r}14,0 \\
1,0\end{array}$ & 0,3 & $\begin{array}{l}13,1 \\
25,3\end{array}$ & $\begin{array}{r}13,6 \\
23,6\end{array}$ & $\begin{array}{r}15,6 \\
20\end{array}$ & $\begin{array}{r}10,5 \\
0,2\end{array}$ & $\begin{array}{r}19,1 \\
29\end{array}$ & $\begin{array}{l}15,1 \\
14,7\end{array}$ & $\begin{array}{l}14, / \\
14,3\end{array}$ & $\begin{array}{l}11,6 \\
128\end{array}$ & $\begin{array}{l}10,1 \\
10,2\end{array}$ & $\begin{array}{l}21,0 \\
127\end{array}$ & $\begin{array}{l}10,1 \\
23,5\end{array}$ & $\begin{array}{l}20,1 \\
11,1\end{array}$ & $\begin{array}{l}18,8 \\
14,6\end{array}$ \\
\hline \multicolumn{23}{|c|}{$\begin{array}{l}\text { Production facultative } \\
\text { Proportion de chaque label }\end{array}$} \\
\hline Avec limites & 27,7 & 35,6 & 38,3 & 37,0 & 31,2 & 34,1 & 36,7 & 41,7 & 48,2 & 36,7 & 38,0 & 51,8 & 48,2 & 44,6 & 39,6 & 34,6 & 37,3 & 33,4 & 32,8 & 34,5 & 43,3 & \\
\hline Sans limites & 70,0 & 62,8 & & 60,3 & 64,0 & & 61,5 & 57,5 & 51,5 & 59,9 & 61,1 & 46,3 & 51,6 & 52,5 & 58,4 & 65,1 & 61,3 & & & & 56,4 & 36,1 \\
\hline Fixe & 64,1 & 68,0 & 77,3 & 75,7 & 84,0 & 63,9 & 68,4 & 79,7 & 86,6 & 63,4 & 67,0 & 84,9 & 79,5 & 72,1 & 78,2 & 75,6 & 73,7 & 63,3 & 65,9 & 75,2 & 68,8 & 81,8 \\
\hline & 33,7 & 30,3 & 20,4 & 21,0 & 11,0 & 33,7 & 29,7 & 19,3 & 13,1 & 33,2 & 32,0 & 13,2 & 20,3 & 25,0 & 19,9 & 24,1 & 24,9 & 35,2 & 33,1 & 23,3 & 30,8 & 17,7 \\
\hline $\begin{array}{l}\text { Avec c. } \\
\text { Avec c. }\end{array}$ & & 0,1 & & & & 0.1 & & 0.1 & & & & & & & & & & & & & & \\
\hline Sans contra & 47,8 & $\begin{array}{r}0,1 \\
43,9\end{array}$ & $\begin{array}{r}0,0 \\
48,1\end{array}$ & $\begin{array}{l}0,1 \\
43,0\end{array}$ & 32,7 & 47,5 & $\begin{array}{r}0,1 \\
43,6\end{array}$ & $\begin{array}{l}0,1 \\
35,2\end{array}$ & & & & $\begin{array}{l}0,0 \\
36,9\end{array}$ & 39,5 & & & & & $\begin{array}{r}0,0 \\
50,5\end{array}$ & $\begin{array}{l}0,0 \\
55,3\end{array}$ & 530 & 450 & $\begin{array}{r}0,1 \\
57,5\end{array}$ \\
\hline & 71,5 & 74,3 & 68,1 & 74,4 & 71,7 & 58,6 & 69,2 & 75,7 & $\begin{array}{l}70,0 \\
76,7\end{array}$ & 51,6 & 55,0 & $\begin{array}{l}70,7 \\
72,7\end{array}$ & 71,3 & $\begin{array}{l}41,4 \\
69,4\end{array}$ & 64,4 & 61,3 & $\begin{array}{l}40,7 \\
68,7\end{array}$ & 56,7 & 59,8 & 53,3 & 60,5 & $\begin{array}{l}51,5 \\
51,5\end{array}$ \\
\hline & 14,0 & 12 & 13,6 & 1,7 & 9,2 & 26 & 17,1 & 9,3 & 9,8 & 9,4 & 7,6 & 9,6 & 10,1 & 8,0 & 7.8 & 9,7 & 6,8 & 14.3 & & $\begin{array}{l}5,1 \\
5,1\end{array}$ & 7,6 & $\begin{array}{l}51,5 \\
15,1\end{array}$ \\
\hline & 12,2 & 12 & 1 & 20,6 & 14,0 & 12 & 11,9 & 14,0 & 13,2 & 13,7 & 13,8 & 15,8 & 18,3 & 19,7 & 13,1 & 14,7 & 11,8 & 18,7 & 21,0 & 18,1 & 20,7 & 18,8 \\
\hline & 16,0 & 14,9 & 10,1 & 9,5 & 10,1 & 11,0 & 8,6 & 6,7 & 5,4 & 27,0 & 27,5 & 6,5 & 7,1 & 10,5 & 17,7 & 22,8 & 21,4 & 7,6 & 12,6 & 6,9 & 7,0 & 8,6 \\
\hline Nbre d'animaux & & & 0,5 & 0,7 & 0,7 & & 0,9 & 1,1 & 1,1 & 1,1 & 1,6 & 1,8 & 1,6 & 1,6 & 1,4 & 1,1 & 1,1 & 2,5 & 11,9 & & 1,5 & $\begin{array}{r}2,0 \\
\end{array}$ \\
\hline Recettes & 11,3 & 10,4 & 0,4 & 0,2 & 1,5 & 16,8 & 14,9 & 7,3 & 8,1 & 8,7 & 8,4 & 8,7 & 9,3 & 7,1 & 13,9 & 16,2 & 13,7 & 16,1 & 4,3 & 20,2 & 0,0 & $\begin{array}{r}15,8 \\
232\end{array}$ \\
\hline & & & & & & & & & & 1,1 & 1,2 & 1,5 & 9,2 & 11,2 & 5,4 & 6,5 & 6,4 & 18,5 & 18,9 & 15,1 & 29,0 & 23,2 \\
\hline $\begin{array}{l}\text { Avec exceppitions } \\
\text { Sans exceptions }\end{array}$ & & & & & & & & & & 21,9 & 22,6 & 0,1 & 0,0 & 0,0 & 12,8 & 14.1 & 11,4 & 8,8 & 11,6 & 22,0 & 10,7 & 14,0 \\
\hline
\end{tabular}


Tableau annexe 12. UE27 : composition de I'ESP par catégorie et étiquette, 1986-2007

Part en pourcentage de l'ESP

\begin{tabular}{|c|c|c|c|c|c|c|c|c|c|c|c|c|c|c|c|c|c|c|c|c|c|c|}
\hline$\overline{\text { PSE (millions EUR) }}$ & $\begin{array}{l}1986 \\
94976\end{array}$ & $\frac{1987}{90007}$ & $\begin{array}{l}1988 \\
83621\end{array}$ & $\frac{1989}{71088}$ & $\begin{array}{l}1990 \\
80125\end{array}$ & $\frac{1991}{96912}$ & $\begin{array}{l}1992 \\
87058\end{array}$ & $\frac{1993}{89346}$ & $\begin{array}{l}1994 \\
89438\end{array}$ & $\begin{array}{l}1995 \\
96907\end{array}$ & $\begin{array}{l}1996 \\
91499\end{array}$ & $\begin{array}{l}1997 \\
94136\end{array}$ & $\begin{array}{l}1998 \\
99338\end{array}$ & $\begin{array}{l}1999 \\
106357\end{array}$ & 2000 & $\begin{array}{ll}2001 \\
93966\end{array}$ & 2002 & 2003 & 2004 & 2005 & 2006 & 2007 \\
\hline \multirow{2}{*}{\multicolumn{23}{|c|}{ Composition par catégo }} \\
\hline Production & & & & & & & & & & & & & & & & & & & & & & \\
\hline Intrants & 4,3 & $\begin{array}{r}1,0 \\
5,1\end{array}$ & $\begin{array}{r}50,0 \\
5,6\end{array}$ & $\begin{array}{l}0,0,0 \\
6.4\end{array}$ & $\begin{array}{r}04,4 \\
7.3\end{array}$ & $\begin{array}{r}0,4 \\
6,3\end{array}$ & $\begin{array}{l}7,7 \\
7,7\end{array}$ & $\begin{array}{r}0,0 \\
6,8\end{array}$ & $\begin{array}{r}60,1 \\
6,5\end{array}$ & $\begin{array}{r}1,4 \\
5,4\end{array}$ & $\begin{array}{r}53,0 \\
6,9\end{array}$ & $\begin{aligned} 5,0 \\
8.4\end{aligned}$ & $\begin{array}{r}0 ., 9 \\
6.2\end{array}$ & 66,7 & 59,6 & $\begin{aligned} 53,8 \\
8,\end{aligned}$ & $\begin{array}{r}58,4 \\
7,5\end{array}$ & $\begin{array}{r}5,9 \\
80\end{array}$ & $\begin{aligned} 56,8 \\
86\end{aligned}$ & $\begin{array}{cc}51,2 \\
99\end{array}$ & 党1,6 & \\
\hline $\mathrm{S} / \mathrm{Na} / \mathrm{Rc} / \mathrm{Rv}$ & 2,8 & 3,6 & 4,1 & 7,0 & 8,2 & 6,9 & 13,1 & 21,2 & 26,8 & 30,4 & 33,0 & 31,6 & 29,8 & $\begin{array}{l}0,3 \\
26,9\end{array}$ & 33,2 & 37,1 & 32,9 & $\begin{array}{l}8,0 \\
33,3\end{array}$ & $\begin{array}{l}8,0 \\
34,1\end{array}$ & $\begin{array}{r}3,9 \\
38,0\end{array}$ & $\begin{array}{r}0,6 \\
46,2\end{array}$ & $\begin{array}{l}12,5 \\
49,7\end{array}$ \\
\hline Superficie & 0,5 & 0,6 & 0,7 & 1,6 & 2,6 & 1,9 & 7,2 & 14,5 & 18,1 & 20,8 & 22,7 & 22,2 & 20,7 & 18,9 & 25,9 & 29,6 & 23,5 & 24,3 & 25,0 & 33,1 & 42,9 & 46,1 \\
\hline $\begin{array}{l}\text { Nore d'anima } \\
\text { Recettes }\end{array}$ & 2,2 & 3,0 & 3,2 & 5,2 & 5,3 & 4,8 & 5,7 & ${ }^{6,6}$ & 8,6 & 9,5 & 10,1 & 9,3 & 9,0 & 7,9 & & 7,5 & 9,3 & 8,6 & 8,8 & 4,5 & 2,7 & 3,1 \\
\hline $\begin{array}{l}\text { Recectes } \\
\text { Revenu }\end{array}$ & $\begin{array}{l}0,1 \\
0,1\end{array}$ & $\begin{array}{l}0,1 \\
0,0\end{array}$ & $\begin{array}{l}0,1 \\
0,0\end{array}$ & $\begin{array}{l}0,1 \\
0,1\end{array}$ & $\begin{array}{l}0,3 \\
0,1\end{array}$ & $\begin{array}{l}0,1 \\
0,1\end{array}$ & $\begin{array}{l}0,1 \\
0,1\end{array}$ & $\begin{array}{l}0,1 \\
0,1\end{array}$ & $\begin{array}{l}0,1 \\
0,0\end{array}$ & $\begin{array}{l}0,0 \\
0,0\end{array}$ & $\begin{array}{l}0,0 \\
0,0\end{array}-$ & $\begin{array}{l}0,0 \\
0,0\end{array}$ & $\begin{array}{l}0,0 \\
0,0\end{array}$ & $\begin{array}{l}0,0 \\
0,0\end{array}$ & $\begin{array}{l}0,2 \\
0,0\end{array}$ & $\begin{array}{l}0,0 \\
0,0\end{array}$ & $\begin{array}{l}0,0 \\
0,0\end{array}$ & $\begin{array}{l}0,3 \\
0,0\end{array}$ & 0,2 & $\begin{array}{l}0,5 \\
0.0\end{array}$ & $\begin{array}{l}0,5 \\
0,0\end{array}$ & $\begin{array}{l}0,5 \\
0,0\end{array}$ \\
\hline Autres produits & 0,4 & $\begin{array}{l}0,0 \\
(0,0)\end{array}$ & 0,5 & $\begin{array}{l}0,1 \\
0,8\end{array}$ & $\begin{array}{l}0,1 \\
0,1\end{array}$ & $\begin{array}{l}0,1 \\
2,5 \\
-1\end{array}$ & $\begin{array}{l}0,1 \\
1,5\end{array}$ & $\begin{array}{l}0,1 \\
1,6 \\
0\end{array}$ & $\begin{array}{l}0,0 \\
0,9\end{array}$ & 2,3 & $\begin{array}{l}0,0 \\
1,2\end{array}$ & 0,3 & 0,1 & $\begin{array}{l}0,0 \\
0,2\end{array}$ & 0,1 & $\begin{array}{l}0,0 \\
1,0\end{array}$ & $\begin{array}{l}, 0,0 \\
1,2\end{array}$ & 0,9 & 0,5 & 0,8 & 1, 0 & $\begin{array}{l}0,0 \\
1,0\end{array}$ \\
\hline Courant & 100,0 & 100,0 & 100,0 & 99,6 & 99,6 & 99,7 & 99,0 & 99,0 & 99,0 & 100,0 & 100,0 & 100,0 & 100,0 & 100,0 & 100,0 & 100,0 & 100,0 & 100,0 & 98,7 & 84,7 & 70,5 & 66,9 \\
\hline Non-courant & & & & 0,4 & 0,4 & 0,3 & 1,0 & 1,0 & 1,0 & 0,0 & 0,0 & 0,0 & 0,0 & 0,0 & 0,0 & 0,0 & 0,0 & 0,0 & 1,3 & 15,3 & 29,5 & \\
\hline Courant/variable & 92,0 & 91,1 & 88,7 & 83,9 & 82,6 & 82,8 & 76,0 & 69,8 & 66,1 & 60,4 & 56,9 & $58,0 \quad 2$ & 62,6 & 65,0 & 57,4 & 51,7 & 57,2 & 56,5 & 54,1 & 47,7 & 41,6 & 37,7 \\
\hline $\begin{array}{l}\text { Couranttfixe } \\
\text { Non-corrant/variable }\end{array}$ & 7,7 & 8,9 & 10,8 & 14,8 & 16,9 & 14,3 & 21,5 & 27,6 & 32,0 & 37,3 & 41,9 & 41,6 & 37,3 & 34,8 & 42,5 & 47,3 & 41,6 & 42,6 & 44,1 & 36,2 & 27,2 & 28,2 \\
\hline $\begin{array}{l}\text { Non-courannivarabiele } \\
\text { Non-courant/fixe }\end{array}$ & & & & & & 0,3 & 1,0 & 1,0 & 1,0 & 0,0 & 0,0 & & & & & & & & & & & \\
\hline Autres prod & 0,4 & $(0,0)$ & 0,5 & 0,8 & 0,1 & 2,5 & 1,5 & 1,6 & 0,9 & 2,3 & 1,2 & 0,3 & 0,1 & 0,2 & 0,1 & 1,0 & 1,2 & 0,9 & 0,5 & 0,8 & $\begin{array}{l}2,0,6 \\
1,6\end{array}$ & 1,0 \\
\hline Production requise & 99,6 & 100,0 & 99,5 & 98,7 & 99,5 & 97,1 & 97,5 & 97,4 & 98,1 & 97,7 & 98,8 & 99,7 & $\begin{array}{lll}99,8 & & \end{array}$ & 99,8 & 99,9 & 99,0 & 98,8 & 99,1 & 98,2 & 83,9 & 68,9 & \\
\hline Un seul produit & 94,3 & 93,8 & 92,7 & 90,1 & 88,8 & 88,2 & 82,8 & 76,9 & 73,7 & 70,0 & 68,5 & 69,6 & 73,1 & 75,0 & 70,2 & 65,5 & 71,1 & 69,5 & 67,7 & 57,2 & 45,6 & 41,1 \\
\hline Un groupe de & 1,6 & 1,8 & 1,8 & 3,0 & 3,4 & 3,0 & 7,3 & 13,5 & 17,5 & 20,3 & 21,4 & 21,0 & 19,0 & 16,7 & 17,4 & 18,6 & 15,4 & & 16,8 & 10,7 & 5,8 & 6,0 \\
\hline $\begin{array}{l}\text { Tous es produits } \\
\text { Production facultative }\end{array}$ & 3,7 & 4,4 & 4,9 & 5,7 & 7,3 & 6,0 & 7,5 & 6,9 & 6,9 & 7,5 & 8,9 & 9,1 & 7,7 & 8,0 & & 15,0 & 12,3 & 13,2 & 13,7 & 16,0 & 17,5 & 18,8 \\
\hline \multicolumn{23}{|l|}{$\frac{\text { Production facultative }}{\text { Proportion de chaque label }}$} \\
\hline $\begin{array}{l}\text { Avec limites } \\
\text { Sec lines }\end{array}$ & 30,0 & 32,2 & 30,2 & 29,2 & 30,0 & 25,4 & 35,4 & 42,7 & 46,8 & 45,6 & 48,3 & 47,5 & 46,8 & 43,9 & 48,8 & 48,2 & 51,1 & 49,4 & 48,6 & 48,3 & 49,1 & 45,3 \\
\hline & & 68,0 & & & & & & & & & & & & & & & & 50,8 & 51,9 & & & 54,7 \\
\hline & $\begin{array}{r}7,7 \\
920\end{array}$ & $\begin{array}{r}8,9 \\
91.1\end{array}$ & $\begin{array}{l}10, \\
88\end{array}$ & $\begin{array}{l}15 \\
89^{2}\end{array}$ & & & & $\begin{array}{l}28,6 \\
69,8\end{array}$ & $\begin{array}{l}33 \\
66\end{array}$ & $\begin{array}{l}37 \\
60\end{array}$ & $\begin{array}{l}41 \\
56\end{array}$ & & 37 & 34,9 & $\begin{array}{l}42,5 \\
57,4\end{array}$ & $\begin{array}{l}47,3 \\
517\end{array}$ & 41 & 42, & 45,3 & 51,5 & 56,8 & $\begin{array}{l}61,3 \\
37,7\end{array}$ \\
\hline & $\begin{array}{r}9,0,0 \\
0,1\end{array}$ & $\begin{array}{r}9,1 \\
0,1\end{array}$ & $\begin{array}{rl}8,1 & 0,1 \\
0.1 & \end{array}$ & $\begin{array}{r}83,9 \\
0,5\end{array}$ & $\begin{array}{r}82,6 \\
0,5\end{array}$ & $\begin{array}{r}8,8 \\
0,4\end{array}$ & $\begin{array}{r}6,0 \\
1,1\end{array}$ & $\begin{array}{r}69,8 \\
2,6\end{array}$ & $\begin{array}{r}66,1 \\
3,7\end{array}$ & $\begin{array}{r}60,4 \\
2,3\end{array}$ & & $\begin{aligned} 58,0 \\
2.4\end{aligned}$ & & $\begin{array}{r}65,0 \\
2,2\end{array}$ & & $\begin{array}{r}1,1, \\
3,\end{array}$ & $\begin{array}{r}5,2 \\
3,0\end{array}$ & $\begin{array}{c}56 \\
3\end{array}$ & & & & $\begin{array}{l}37,7 \\
36,9\end{array}$ \\
\hline & 1,3 & $\begin{array}{l}0,1 \\
1,2\end{array}$ & 1,5 & 2,9 & $\begin{array}{l}0,0 \\
3,1\end{array}$ & $\begin{array}{l}0,4 \\
3,3\end{array}$ & 5,1 & $\begin{array}{l}<, 0 \\
7,3\end{array}$ & $\begin{array}{l}3,1 \\
8,9\end{array}$ & $\begin{array}{l}2,5 \\
10,5\end{array}$ & 11, & $\begin{array}{l}2,4,2 \\
12,2\end{array}$ & 11, & $\begin{array}{l}<, 2 \\
10,5\end{array}$ & $\begin{array}{r}3,0 \\
13,7\end{array}$ & 14 & $\begin{array}{l}3,0 \\
13,0\end{array}$ & 13, & $\begin{array}{l}10,2 \\
13,2\end{array}$ & $\begin{array}{l}12,3 \\
12,3\end{array}$ & $\begin{array}{l}\text { sw, } \\
12,7\end{array}$ & $\begin{array}{l}3,1,2 \\
11,2\end{array}$ \\
\hline & 10,7 & 12,9 & 15,1 & 20 & & 17,0 & 20 & 22,7 & 24,4 & 27,8 & 31 & & 27 & & 29 & & 29 & & & 16 & & 15,8 \\
\hline$u$ & $\begin{array}{r}94,3 \\
16\end{array}$ & $\begin{array}{r}93,8 \\
1,8\end{array}$ & $\begin{array}{r}92,7 \\
1,8\end{array}$ & $\begin{array}{r}90,1 \\
3.0\end{array}$ & $\begin{array}{r}88,8 \\
34\end{array}$ & $\begin{array}{r}88,2 \\
3,0\end{array}$ & $\begin{array}{l}82,8 \\
73\end{array}$ & $\begin{array}{r}76,9 \\
135\end{array}$ & $\begin{array}{l}73,7 \\
117\end{array}$ & 70,0 & 68,5 & 69 & 73,1 & 75,0 & 70, & 6 & 71 & 69 & 67,7 & 57,2 & 45,6 & $\begin{array}{r}41,1 \\
6,0\end{array}$ \\
\hline es produits & $\begin{array}{l}1,6 \\
3,7\end{array}$ & $\begin{array}{l}1,6 \\
4,4\end{array}$ & $\begin{array}{l}1,6 \\
4,9\end{array}$ & $\begin{array}{l}5,0 \\
5,7\end{array}$ & $\begin{array}{l}3,4 \\
7,3\end{array}$ & 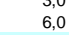 & $\begin{array}{l}7,3 \\
7,5\end{array}$ & $\begin{array}{r}15,5 \\
6,9\end{array}$ & $\begin{array}{r}\begin{array}{r}17,5 \\
6,9\end{array} \\
6\end{array}$ & $\begin{array}{r}20,3 \\
7,5\end{array}$ & $\begin{array}{r}21,4 \\
8,9\end{array}$ & $\begin{array}{c}21,0 \\
9,1\end{array}$ & $\begin{array}{c}\begin{array}{l}19,0 \\
7,7\end{array} \\
-\end{array}$ & 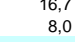 & $\begin{array}{l}\text { 17, } \\
12,\end{array}$ & $\begin{array}{l}18 \\
15 \\
15\end{array}$ & $\begin{array}{l}15 \\
12\end{array}$ & $\begin{array}{l}16 \\
13\end{array}$ & $\begin{array}{l}\begin{array}{l}16, \\
13,\end{array} \\
\text { (1) }\end{array}$ & $\begin{array}{l}\begin{array}{l}10,7 \\
16,0\end{array}\end{array}$ & & $\begin{array}{l}0,0 \\
18,8\end{array}$ \\
\hline Superficie & 0,5 & 0,6 & & 1,6 & 2,6 & 1,9 & 7,2 & 14,5 & 18,1 & 20,8 & 22, & 22,2 & 20,7 & 18,9 & 25,9 & 29,6 & 23 & & $25, \mathrm{C}$ & 33,1 & & 461 \\
\hline re d'animaux & 2,2 & 3,0 & 3,2 & 5,2 & 5,3 & 4,8 & 5,7 & 6,6 & 8,6 & 9,5 & 10,1 & 9,3 & 9,0 & 7,9 & 7,1 & 7 & 9 & & 8,8 & 4,5 & & \\
\hline $\begin{array}{l}\text { Recettes } \\
\text { Revenu }\end{array}$ & 0,1 & $\begin{array}{l}0,1 \\
0,0\end{array}$ & $\begin{array}{l}0,1 \\
0,0\end{array}$ & $\begin{array}{l}0,1 \\
0,1\end{array}$ & $\begin{array}{l}0,3 \\
0,\end{array}$ & $\begin{array}{l}0,1 \\
0.1\end{array}$ & $\begin{array}{l}0,1 \\
0,1\end{array}$ & $\begin{array}{l}0,1 \\
0,1\end{array}$ & $\begin{array}{l}0,1 \\
0\end{array}$ & $\begin{array}{l}0,0 \\
0.0\end{array}$ & $\begin{array}{l}0,0 \\
0,0\end{array}$ & $\begin{array}{l}0,0 \\
0.0\end{array}$ & $\begin{array}{l}0,0 \\
0,0\end{array}$ & $\begin{array}{l}0,0 \\
0,0\end{array}$ & $\begin{array}{l}0,2 \\
0,0\end{array}$ & 0,0 & 0,0 & 0 & 0,2 & 0,5 & & \\
\hline $\begin{array}{l}\text { Revenu } \\
\text { Avec exceptions }\end{array}$ & 0,1 & 0,0 & 0,0 & & & & & & & & & & & & & & & & 0,0 & 0,0 & 0,0 & 21,3 \\
\hline Sans exceptions & & - & & 0,4 & 0,4 & 0,3 & 1,0 & 1,0 & 1,0 & 0,0 & 0,0 & 0,0 & 0,0 & 0,0 & 0,0 & 0,0 & 0,0 & 0,0 & 1,3 & 7,7 & $\begin{array}{r}9,4 \\
9,4\end{array}$ & \\
\hline
\end{tabular}


Tableau annexe 13. Islande : composition de l'ESP par catégorie et étiquette, 1986-2007

Part en pourcentage de l'ESP

\begin{tabular}{|c|c|c|c|c|c|c|c|c|c|c|c|c|c|c|c|c|c|c|c|c|c|c|}
\hline & 1986 & 1987 & 1988 & 1989 & 1990 & 1991 & 1992 & 1993 & 1994 & 1995 & 1996 & 1997 & 1998 & 1999 & 2000 & 2001 & 2002 & 2003 & 2004 & 2005 & 2006 & $\frac{2007}{13610}$ \\
\hline PSE (millions ISK) & 6506 & 8342 & 8860 & 10200 & 10914 & 11866 & 10996 & 9678 & 8879 & 9025 & 8764 & 9171 & 12921 & 13278 & 10964 & 11686 & 14277 & 13397 & 13829 & 15515 & 15057 & \\
\hline \multicolumn{23}{|c|}{ Composition par catégorie avec labels correspondants } \\
\hline Production & 91,7 & & 91,0 & 88,2 & 90,9 & 93,1 & 94,2 & 95,1 & 95,1 & 95,9 & 78,6 & 78,3 & 82,9 & 84,0 & 80,1 & 72,0 & 80,4 & 77,8 & 78,1 & 78,3 & 78,4 & 72,99 \\
\hline $\begin{array}{l}\text { Intrants } \\
\mathrm{S} / \mathrm{Na} / \mathrm{RC} / \mathrm{Rv}\end{array}$ & 8,3 & 6,6 & 8,1 & 10,9 & 8,3 & ${ }^{6,1}$ & 5,4 & 4,8 & 4,9 & 4,1 & 4,4 & 4,4 & 4,8 & 3,7 & 4,5 & 4,9 & $\begin{array}{r}4,2 \\
152\end{array}$ & 5,3 & 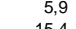 & 7,2 & 4,7 & 5,2 \\
\hline Superficie & & & & & 0,8 & & & 0,1 & & & 16,9 & 17,3 & 12,3 & 12,3 & 15,3 & 23,1 & 15,2 & 16,0 & 15,4 & 14,5 & 16,7 & 22,0 \\
\hline Nbre d'animaux & - & 0,7 & 0,9 & 0,9 & 0,8 & 0,8 & 0,4 & 0,1 & 0,0 & - & 16,9 & 17,3 & 12,3 & 12,3 & 15,3 & 23,1 & 15,2 & 16,0 & 15,4 & 14,5 & 16,7 & 22,0 \\
\hline $\begin{array}{l}\text { Recettes } \\
\text { Revenu }\end{array}$ & : & - & - & $\therefore$ & & 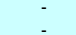 & & - & - & : & & 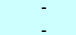 & & & & & & & & & & \\
\hline Autres produits & & & & & & & & & & & & & & & & & 0,2 & 0,9 & 0,7 & 0,1 & 0,3 & \\
\hline Courant & 100,0 & 99,3 & 99,1 & 99,1 & 99,2 & 99,2 & 99,6 & 99,9 & 100,0 & 100,0 & 83,1 & 82,7 & 87,7 & 87,7 & 84,7 & 76,9 & 84,8 & 84,0 & 84,6 & 85,5 & 83,6 & 81,4 \\
\hline Non-courant & & 0,7 & 0,9 & 0,9 & 0,8 & 0,8 & 0,4 & 0,1 & 0,0 & & 16,9 & 17,3 & 12,3 & 12,3 & 15,3 & 23,1 & 15,2 & 16,0 & 15,4 & 14,5 & 16,4 & 18,6 \\
\hline Courant/variable & 94,3 & 94,1 & 89,8 & 86,3 & 90,8 & 91,2 & 76,2 & 49,4 & 49,8 & 51,0 & 49,0 & 49,0 & 60,4 & 60,9 & 50,6 & 42,3 & 52,3 & 47,2 & 47,6 & 50,2 & 48,9 & $\begin{array}{l}41,8 \\
39,7\end{array}$ \\
\hline $\begin{array}{l}\text { Courant/fixe } \\
\text { Non-courant/variable }\end{array}$ & 5,7 & 5,2 & 9,3 & 12,8 & 8,4 & 8,1 & 23,4 & 50,4 & 50,2 & 49,0 & 34,0 & 33,7 & 27,3 & 26,8 & 34,1 & 34,6 & 32,3 & 35,8 & 36,4 & 35,3 & 34,4 & 39,7 \\
\hline $\begin{array}{l}\text { Non-courant/fixe } \\
\text { Autres produits }\end{array}$ & - & 0,7 & 0,9 & 0,9 & 0,8 & 0,8 & 0,4 & 0,1 & 0,0 & - & 16,9 & 17,3 & 12,3 & 12,3 & 15,3 & 23,1 & 15,2 & 16,0 & 15,4 & 14,5 & 16,4 & 18,6 \\
\hline Production requise & 100,0 & 99,3 & $\begin{array}{lll}99,1 & \end{array}$ & $\begin{array}{lll}99,1 & \end{array}$ & 99,2 & 99,2 & $\begin{array}{ll}99,6 \\
\end{array}$ & 99,9 & 100,0 & 100,0 & 100,0 & 99,5 & $\begin{array}{lll}99,7 & \end{array}$ & 99,8 & $\begin{array}{lll}99,9 \\
\end{array}$ & $\begin{array}{lll}91,7 & \end{array}$ & 97,5 & 98,7 & 99,0 & 99,5 & 99,7 & 100,0 \\
\hline Un seul produit & 91,7 & 92,7 & 91,0 & 88,2 & 90,9 & 93,1 & 94,2 & 95,1 & 95,2 & 96,1 & 95,7 & 95,3 & 95,1 & 96,2 & 95,6 & 86,9 & 93,3 & 93,5 & 93,1 & 92,4 & 95,0 & 94,8 \\
\hline $\begin{array}{l}\text { Un groupe de produits } \\
\text { Tous les produtits }\end{array}$ & 4,3 & 4,4 & 6,9 & 9,6 & 7,0 & 4,7 & 4,3 & 3,5 & 3,5 & 2,8 & 3,5 & 3,3 & 4,0 & 2,9 & 3,5 & 3,5 & 2,7 & 3,2 & 4,1 & 4,4 & 1,5 & 1,6 \\
\hline $\begin{array}{l}\text { Tous les produits } \\
\text { Production facultative }\end{array}$ & 4,0 & $\begin{array}{l}2,2 \\
0,7\end{array}$ & $\begin{array}{l}1,2 \\
0,9\end{array}$ & $\begin{array}{l}1,3 \\
0,9\end{array}$ & 1,3 & $\begin{array}{l}1,4 \\
0,8\end{array}$ & $\begin{array}{l}1,2 \\
0,4\end{array}$ & $\begin{array}{l}1,3 \\
0,1\end{array}$ & 1,4 & 1,2 & 1,0 & $\begin{array}{l}1,1 \\
0,5\end{array}$ & 0,8 & 0,8 & $\begin{array}{l}1,0 \\
01\end{array}$ & $\begin{array}{l}1,4 \\
8,3\end{array}$ & $\begin{array}{r}1,5 \\
25\end{array}$ & 2,0 & 1,8 & $\begin{array}{l}2,8 \\
0.5\end{array}$ & $\begin{array}{l}3,2 \\
0,3\end{array}$ & 3,6 \\
\hline \multicolumn{23}{|c|}{ Proportion de chaque label } \\
\hline Avec limites & 0,4 & 0,6 & 0,7 & 0,6 & 0,6 & 0,4 & 16,1 & 44,3 & 45,5 & 45,3 & 47,2 & 46,5 & 35,0 & 35,7 & 45,5 & 45,1 & 40,1 & 45,3 & 44,4 & 41,2 & 45,3 & 51,5 \\
\hline Sans limites & 99,6 & 99,4 & 99,3 & 99,4 & 99,4 & 99,6 & 83,9 & 55,7 & 54,5 & 54,7 & 52,8 & 53,5 & 65,0 & 64,3 & 54,5 & 54,9 & 59,9 & 54,7 & 55,6 & 58,8 & 54,7 & 48,5 \\
\hline $\begin{array}{l}\text { Fixe } \\
\text { Variable }\end{array}$ & 5,7 & 5,9 & $\begin{array}{l}10,2 \\
898\end{array}$ & 13,7 & 9,2 & 8,8 & 23,8 & 50,6 & 50,2 & 49,0 & 51,0 & 51,0 & 39,6 & 39,1 & 49,4 & 57,7 & $\begin{array}{l}47,5 \\
523\end{array}$ & 51,9 & 51,8 & 49,8 & 50,8 & 58,2 \\
\hline \multirow{2}{*}{\multicolumn{6}{|c|}{$\begin{array}{l}\text { Variable } \\
\text { Avec c. (obligatoire) } \\
\text { Avec c. (volontaire) }\end{array}$}} & & 6,2 & 49,4 & 49,8 & 51,0 & 49,0 & 49,0 & 60,4 & 60,9 & 50,6 & 42,3 & 52,3 & 47,2 & 47,6 & 50,2 & 48,9 & \\
\hline & & & & & & & & & & & & & & & & & 0,2 & 0,9 & 0,7 & 0,1 & 0,3 & \\
\hline Sans contrainte & 8,3 & 7,3 & 11,3 & 13,9 & 11,1 & 8,8 & 23,8 & 50,6 & 50,2 & 49,0 & 51,0 & 51,0 & 39,6 & 39,1 & 49,4 & 57,7 & & 51,9 & 51,8 & 49,8 & 50,8 & 58,2 \\
\hline Un seul p & 91,7 & 92,7 & 91,0 & 88,2 & 90,9 & 93,1 & 94,2 & 95,1 & 95,2 & 96,1 & 95,7 & 95,3 & 95,1 & 96,2 & 95,6 & 86,9 & 93,3 & 93,5 & 93,1 & 92,4 & 95,0 & 94,8 \\
\hline $\begin{array}{l}\text { Un groupe de produits } \\
\text { Tous les produits }\end{array}$ & $\begin{array}{l}4,3 \\
40\end{array}$ & $\begin{array}{r}4,4 \\
2,2\end{array}$ & $\begin{array}{l}6,9 \\
1,2\end{array}$ & $\begin{array}{l}9,6 \\
1,3\end{array}$ & $\begin{array}{l}7,0 \\
13\end{array}$ & $\begin{array}{l}4,7 \\
1,4\end{array}$ & $\begin{array}{l}4,3 \\
1,2\end{array}$ & $\begin{array}{l}3,5 \\
1,3\end{array}$ & $\begin{array}{r}3,5 \\
14\end{array}$ & $\begin{array}{l}2,8 \\
1,2\end{array}$ & $\begin{array}{l}3,5 \\
1,0\end{array}$ & $\begin{array}{l}3,3 \\
1,\end{array}$ & $\begin{array}{r}4,0 \\
08\end{array}$ & $\begin{array}{l}2,9 \\
0.8\end{array}$ & $\begin{array}{l}3,5 \\
10\end{array}$ & $\begin{array}{l}3,5 \\
14\end{array}$ & $\begin{array}{l}2,7 \\
15\end{array}$ & $\begin{array}{l}3,2 \\
20\end{array}$ & $\begin{array}{l}4,1 \\
18\end{array}$ & $\begin{array}{r}4,4 \\
28\end{array}$ & $\begin{array}{r}1,5 \\
32\end{array}$ & $\begin{array}{l}1,6 \\
36\end{array}$ \\
\hline Superficie & & & & & & & & & & & & & & & & & & & & & & \\
\hline $\begin{array}{l}\text { Nbre d'animaux } \\
\text { Recettes }\end{array}$ & : & 0,7 & 0,9 & 0,9 & 0,8 & 0,8 & 0,4 & 0,1 & 0,0 & $\therefore$ & 16,9 & 17,3 & 12,3 & 12,3 & 15,3 & 23,1 & 15,2 & 16,0 & 15,4 & 14,5 & 16,7 & 22,0 \\
\hline & & & 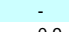 & & & & & & & & & & & & & & & & & & & \\
\hline $\begin{array}{l}\text { Avece exceptions } \\
\text { Sans exceptions }\end{array}$ & & 0,7 & 0,9 & 0,9 & 0,8 & 0,8 & 0,4 & 0,1 & , o 0 & & 0,0 & o,5 & 0,3 & 0,2 & 0,1 & 8,3 & 2,3 & 0,4 & 0,4 & 0,4 & & \\
\hline
\end{tabular}


Tableau annexe 14. Japon : composition de l'ESP par catégorie et étiquette, 1986-2007

Part en pourcentage de l'ESP

\begin{tabular}{|c|c|c|c|c|c|c|c|c|c|c|c|c|c|c|c|c|c|c|c|c|c|c|}
\hline & 1986 & 1987 & 1988 & 1989 & 1990 & 1991 & 1992 & 1993 & 1994 & 1995 & 1996 & 1997 & 1998 & 1999 & 2000 & 2001 & 2002 & 2003 & 2004 & 2005 & 2006 & 2007 \\
\hline$\overline{\text { PSE (milliards JPY) }}$ & 7726 & 7135 & 6845 & 6564 & 6190 & 6159 & 6686 & 6339 & 7415 & 6841 & 6254 & 5607 & 5991 & 5906 & 5804 & 5376 & 5511 & 5462 & 5191 & 4908 & 4566 & 4149 \\
\hline \multicolumn{23}{|c|}{ Composition par catégorie avec labels correspondants } \\
\hline Production & 92,9 & 92,7 & 92,5 & 92,3 & 92,7 & 92,6 & 93,3 & 93,3 & 95,0 & 94,1 & 93,2 & 92,5 & 93,8 & 93,9 & 93,6 & 93,2 & 93,3 & 93,5 & 93,9 & 93,1 & 92,9 & \\
\hline Intrants & 3,9 & 4,3 & 4,3 & 4,2 & 4,5 & 4,6 & 4,5 & 5,1 & 4,0 & 4,6 & 4,7 & 5,1 & 4,3 & 4,2 & 3,6 & 3,4 & 3,0 & 2,8 & 2,9 & 2,8 & 2,8 & $\begin{array}{c}4,4 \\
4,4\end{array}$ \\
\hline $\mathrm{S} / \mathrm{Na} / \mathrm{Rc} / \mathrm{Rv}$ & 3,2 & 3,0 & 3,2 & 3,5 & 2,8 & 2,8 & 2,2 & 1,6 & 1,0 & 1,3 & 2,1 & 2,4 & 1,9 & 2,0 & 2,8 & 3,4 & 3,6 & 3,7 & 3,3 & 4,1 & 4,3 & 6,8 \\
\hline $\begin{array}{l}\text { Superficie } \\
\text { Nbre danimaux }\end{array}$ & 3,2 & 3,0 & 3,2 & 3,5 & 2,8 & 2,8 & 2,2 & 1,6 & 1,0 & 1,3 & 2,1 & 2,4 & 1,9 & 2,0 & 2,8 & 3,4 & 3,6 & 3,7 & 3,3 & 3,8 & 4,1 & 6,7 \\
\hline Recettes & - & - & . & - & - & . & . & - & - & - & - & - & - & - & - & - & - & - & - & - & & \\
\hline $\begin{array}{l}\text { Revenu } \\
\text { Autres produits }\end{array}$ & & & & & & & & & & & & & & & & & & & & 0,2 & 0,2 & \\
\hline $\begin{array}{l}\text { Aurres produrts } \\
\text { Courant }\end{array}$ & 96,8 & 97,0 & 96,8 & 96,5 & 97,2 & 97,2 & 97,8 & 98,4 & 99,0 & 98,7 & 97,9 & 97,6 & 98,1 & 98,0 & 97,2 & 96,6 & 96,4 & 96,3 & 96,9 & 96,5 & 96,2 & 93,6 \\
\hline Non-courant & 3,2 & 3,0 & 3,2 & 3,5 & 2,8 & 2,8 & 2,2 & 1,6 & 1,0 & 1,3 & 2,1 & 2,4 & 1,9 & 2,0 & 2,8 & 3,4 & 3,6 & 3,7 & 3,1 & 3,5 & 3,8 & 6,4 \\
\hline Courant/variable & 93,1 & 93,0 & 92,9 & 92,7 & 93,4 & 93,3 & 94,0 & 94,2 & 95,7 & 94,8 & 93,9 & 93,3 & 94,4 & 94,5 & 92,7 & 92,1 & 92,0 & 91,6 & 92,1 & 91,5 & 91,2 & $\begin{array}{c}88,2 \\
53\end{array}$ \\
\hline $\begin{array}{l}\text { Courantstife } \\
\text { Non-courant/variable }\end{array}$ & 3,7 & 4,0 & 3,9 & 3,8 & 3,9 & 3,9 & 3,8 & 4,2 & 3,3 & 3,9 & 3,9 & & 3,6 & 3,5 & 4,5 & 4,5 & 4,3 & 4,7 & 4,8 & & 5,1 & \\
\hline $\begin{array}{l}\text { Non-courantffixe } \\
\text { Autres produits }\end{array}$ & 3,2 & 3,0 & 3,2 & 3,5 & 2,8 & 2,8 & 2,2 & 1,6 & 1,0 & 1,3 & 2,1 & 2,4 & 1,9 & 2,0 & 2,8 & 3,4 & 3,6 & 3,7 & 3,1 & 3,5 & 3,8 & 6,4 \\
\hline Production requise & 96,8 & 97,0 & 96,8 & 96,5 & 97,2 & 97,2 & 97,8 & 98,4 & 99,0 & 98,7 & 97,9 & 97,6 & 98,1 & 98,0 & 97,2 & 96,6 & 96,4 & 96,3 & 96,9 & 96,5 & 96,2 & 93,6 \\
\hline Un seul produit & 92,9 & 92,7 & 92,5 & 92,3 & 92,7 & 92,6 & 93,3 & 93,3 & 95,0 & 94,1 & 93,2 & 92,5 & 93,8 & 93,9 & 93,6 & 93,2 & 93,3 & 93,5 & 93,9 & 93,4 & 93,1 & \\
\hline Un groupe de produits & 0,0 & 0,0 & 0,0 & 0,0 & 0,0 & 0,0 & 0,0 & 0,1 & 0,0 & 0,0 & 0,0 & 0,1 & 0,1 & 0,1 & 0,1 & 0,1 & 0,1 & 0,1 & 0,2 & 0,4 & 0,4 & 0,2 \\
\hline Tous les produits & 3,9 & 4,2 & 4,2 & 4,2 & 4,5 & 4,6 & 4,5 & 5,0 & 4,0 & 4,6 & 4,6 & 5,1 & 4,2 & 4,1 & 3,5 & 3,3 & 2,9 & 2,7 & 2,8 & 2,8 & 2,7 & 4,3 \\
\hline \multicolumn{23}{|c|}{$\begin{array}{l}\text { Prododution facultative } \\
\text { Proportion de chaque label }\end{array}$} \\
\hline $\begin{array}{l}\text { Proportion de chaque ia } \\
\text { Avec limites }\end{array}$ & 2.1 & & & & & & & & 1,6 & & & & 2.1 & & 3,0 & 3,1 & 2.8 & 2,3 & 2,2 & & 2,6 & \\
\hline Sans limites & 97,9 & 98 & 97,9 & 97,5 & 97 & 97,3 & 97,6 & 97,4 & 98,4 & 97,9 & 98,3 & 97,3 & 97,9 & 97,7 & 97,0 & 96,9 & 97,2 & 97,7 & 97,8 & 97,7 & 97,4 & 97,9 \\
\hline Fixe & 6,9 & 7,0 & 7,1 & 7,3 & 6 & 6,7 & 6,0 & 5,8 & 4,3 & 5,2 & 6,1 & 6,7 & 5,6 & 5,5 & 7,3 & 7,9 & 8,0 & 8,4 & 7,9 & 8,5 & 8,8 & 11,8 \\
\hline \multirow{2}{*}{\multicolumn{23}{|c|}{$\begin{array}{l}\text { Avec c. (obligatoire) } \\
\text { Avec c. (uolontaire) }\end{array}$}} \\
\hline & & & & & & & & & & & & & & & & & & & & & & \\
\hline Sans contrainte & 9,9 & 10,0 & 11,2 & 10,6 & 10,6 & 10,6 & 9,8 & 10,2 & 7,8 & 9,0 & 8.9 & 10,6 & 8.9 & 9,3 & 10,9 & 12,2 & 11,8 & 11,8 & 11,2 & 11,6 & 12,2 & $\begin{array}{r}4,1 \\
10,8\end{array}$ \\
\hline Un seul produit & 92,9 & 92,7 & 92,5 & 92,3 & 92,7 & 92,6 & 93,3 & 93,3 & 95,0 & 94,1 & 93,2 & 92,5 & 93,8 & 93,9 & 93,6 & 93,2 & 93,3 & 93,5 & 93,9 & 93,4 & 93,1 & 89,0 \\
\hline Un groupe de & 0,0 & 0,0 & 0,0 & 0,0 & 0,0 & 0,0 & 0,0 & 0,1 & 0,0 & 0,0 & 0,0 & 0,1 & 0,1 & 0,1 & 0,1 & 0,1 & 0,1 & 0,1 & 0,2 & 0,4 & 0,4 & 0,2 \\
\hline Tous les produits & 3,9 & 4,2 & 4,2 & 4,2 & 4,5 & 4,6 & 4,5 & 5,0 & 4,0 & 4,6 & 4,6 & 5,1 & 4,2 & 4,1 & 3,5 & 3,3 & 2,9 & 2,7 & 2,8 & 2,8 & 2,7 & $\begin{array}{l}4,3 \\
6,7\end{array}$ \\
\hline $\begin{array}{l}\text { Superficie } \\
\text { Nrbe }\end{array}$ & 3,2 & 3,0 & 3,2 & 3,5 & 2,8 & 2,8 & 2,2 & 1,6 & 1,0 & 1,3 & 2,1 & 2,4 & 1,9 & 2,0 & 2,8 & 3,4 & 3,6 & 3,7 & 3,3 & 3,8 & 4,1 & 6,7 \\
\hline $\begin{array}{l}\text { Nore dianimaux } \\
\text { Recettes }\end{array}$ & - & - & - & - & - & - & - &. & - & - & - & . & - & . & & & & & & & & \\
\hline $\begin{array}{l}\text { Revenu } \\
\text { Avec exceptions }\end{array}$ & 3,2 & 3,0 & 3,2 & 3,5 & 2,8 & 2,8 & 2,2 & 1,6 & 1,0 & 1,3 & 2,1 & 2,4 & 1,9 & 2,0 & & & & 3,3 & & $\begin{array}{l}0,2 \\
3,0\end{array}$ & $\begin{array}{l}0,2 \\
3,3\end{array}$ & $\begin{array}{l}0,0 \\
3,6\end{array}$ \\
\hline $\begin{array}{l}\text { Avec exceppitions } \\
\text { Sans exceptions }\end{array}$ & , & 3,0 & 3,2 & , & , , & 2,8 & , 2, & , & & & & & , & 2,0 & $\begin{array}{l}2,2 \\
0,6\end{array}$ & $\begin{array}{l}2,8 \\
0,6\end{array}$ & $\begin{array}{l}3,0 \\
0,6\end{array}$ & $\begin{array}{l}3,3 \\
0,4\end{array}$ & $\begin{array}{l}2,8 \\
0,3\end{array}$ & $\begin{array}{l}3,0 \\
0,5\end{array}$ & $\begin{array}{l}0,5 \\
0,5\end{array}$ & \\
\hline
\end{tabular}




\section{Tableau annexe 15. Corée : composition de l'ESP par catégorie et étiquette, 1986-2007}

\section{Part en pourcentage de l'ESP}

\begin{tabular}{|c|c|c|c|c|c|c|c|c|c|c|c|c|c|c|c|c|c|c|c|c|c|c|}
\hline & 1986 & 1987 & 1988 & 1989 & 1990 & 1991 & 1992 & 1993 & 1994 & 1995 & 1996 & 1997 & 1998 & 1999 & 2000 & 2001 & 2002 & 2003 & 2004 & 2005 & 2006 & 2007 \\
\hline 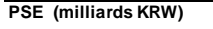 & 8290 & 9047 & 11527 & 12303 & 13569 & 14609 & 15261 & 15619 & 17755 & 19361 & 18735 & 19147 & 17300 & 21412 & 21728 & 20453 & 21747 & 20492 & 23445 & 24096 & 24064 & 23665 \\
\hline \multicolumn{23}{|c|}{ Composition par catégorie avec labels correspondants } \\
\hline Production & & 98,8 & 98,9 & 98,5 & 96,2 & 96,0 & 95,4 & 95,2 & 94,8 & 94,7 & 94,3 & 94,1 & 94,4 & 95,9 & 95,6 & 94,0 & 93,1 & 91,4 & 93,4 & 88,8 & 90,4 & 90,9 \\
\hline $\begin{array}{l}\text { Intrants } \\
S / N / B / B V\end{array}$ & 0,3 & 0,7 & 0,9 & $\begin{array}{l}1,3 \\
02\end{array}$ & 1,9 & $\begin{array}{l}2,1 \\
19\end{array}$ & 2,5 & 3,1 & $\begin{array}{l}3,5 \\
17\end{array}$ & 4,1 & 4,5 & 4,9 & 4,7 & $\begin{array}{l}3,2 \\
10\end{array}$ & $\begin{array}{l}2,6 \\
18\end{array}$ & 2,7 & 2,9 & 2,5 & 2,1 & 2,2 & 2,2 & $\begin{array}{l}2,7 \\
63\end{array}$ \\
\hline Superficie & & & & & & & & & & & & & & 0,0 & $\begin{array}{l}, 0 \\
0,0\end{array}$ & $\begin{array}{l}0,4 \\
1,0\end{array}$ & $\begin{array}{l}4,0 \\
1,8\end{array}$ & $\begin{array}{l}2,0 \\
2,7\end{array}$ & 2,4 & $\begin{array}{l}6,0 \\
6,6\end{array}$ & $\begin{array}{l}5,1 \\
5,1\end{array}$ & $\begin{array}{l}6,3 \\
4,4\end{array}$ \\
\hline Nbre d'animaux & & & 0,0 & & 0,0 & & & 0,0 & 0,1 & 0,1 & 0,0 & 0,1 & 0,1 & 0,0 & 0,1 & 0,3 & 0,3 & 0,4 & 0,1 & 0,1 & 0,1 & 0,0 \\
\hline Recettes & 0,0 & 0,3 & 0,2 & 0,1 & 0,1 & 0,0 & 0,1 & 0,1 & 0,5 & 0,2 & 0,1 & 0,1 & 0,3 & 0,5 & 0,3 & 0,1 & 0,6 & 0,5 & 0,2 & 0,1 & 0,0 & 0,1 \\
\hline Revenu & 0,2 & 0,2 & 0,1 & 0,1 & 1,8 & 1,9 & 2,0 & 1,5 & 1,2 & 1,0 & 1,1 & 0,8 & 0,5 & 0,4 & 1,3 & 2,0 & 1,3 & 2,4 & 1,8 & 2,2 & 2,2 & 1,9 \\
\hline Autres produits & & & & & 0,1 & 0,0 & 0,0 & 0,0 & & & & 0,0 & 0,0 & 0,0 & 0,0 & 0,0 & 0,0 & 0,0 & 0,1 & 0,0 & & \\
\hline $\begin{array}{l}\text { Courant } \\
\text { Non-courant }\end{array}$ & 100,0 & 100,0 & 100,0 & 100,0 & 100,0 & 100,0 & 100,0 & 100,0 & 100,0 & 100,0 & 100,0 & 100,0 & 100,0 & 100,0 & 100,0 & 100,0 & 100,0 & 100,0 & $\begin{array}{r}100,0 \\
0,0\end{array}$ & $\begin{array}{r}97,5 \\
2,5\end{array}$ & $\begin{array}{r}96,9 \\
3,1\end{array}$ & $\begin{array}{r}96,9 \\
3,1\end{array}$ \\
\hline Courant/variable & 99,5 & 99,1 & 99,0 & 98,6 & 96,2 & 96,0 & 95,5 & 95,3 & 95,3 & 94,9 & 94,4 & 94,2 & 94,7 & 96,4 & 95,9 & 94,1 & 93,7 & 91,9 & 93,6 & 88,9 & 90,4 & $\begin{array}{l}2,1 \\
91,0\end{array}$ \\
\hline $\begin{array}{l}\text { Courantfixe } \\
\text { Cone }\end{array}$ & 0,5 & 0,9 & $\begin{array}{l}1,0 \\
1,0\end{array}$ & $\begin{array}{r}0,4 \\
1,4\end{array}$ & 3,7 & 4,0 & 4,5 & 4,7 & 4,7 & 5,1 & 5,6 & 5,8 & 5,3 & 3,6 & 4,1 & 5,9 & 6,3 & 8,0 & 6,3 & $\begin{array}{r}8,5 \\
8,5\end{array}$ & 6,5 & 5,9 \\
\hline Non-courant/fixe & - & - & - & - & & & & & - & - & & & & & & & & & 0,0 & 2,5 & 3,1 & 3,1 \\
\hline $\begin{array}{l}\text { Autres produits } \\
\text { Production reguvise }\end{array}$ & & & & & 0,1 & 0,0 & 0,0 & 0,0 & & & & 0,0 & 0,0 & 0,0 & 0,0 & 0,0 & 0,0 & 0,0 & 0,1 & 0,0 & & \\
\hline $\begin{array}{l}\text { Production requise } \\
\text { Un seul produit }\end{array}$ & 100,0 & $\begin{array}{l}100,0 \\
988\end{array}$ & $\begin{array}{l}100,0 \\
989\end{array}$ & 100,0 & $\begin{aligned} 99,9 \\
962\end{aligned}$ & $\begin{array}{l}100,0 \\
960\end{array}$ & $\begin{array}{r}100,0 \\
954\end{array}$ & $\begin{array}{l}100,0 \\
0.95\end{array}$ & 100,0 & 100,0 & 100,0 & 100,0 & 100,0 & 100,0 & 100,0 & 100,0 & 100,0 & 100,0 & 99,9 & 97,4 & 96,9 & 96,9 \\
\hline $\begin{array}{l}\text { Un seul produit } \\
\text { Un groupe de produits }\end{array}$ & $\begin{array}{r}99,5 \\
9\end{array}$ & 98,8 & 98,9 & $\begin{array}{c}98,5 \\
0,5\end{array}$ & 96,2 & 96,0 & 95,4 & 95,2 & 94,8 & 94,7 & 94,3 & 94,1 & 94,4 & 95,9 & 95,6 & 94,0 & $\begin{array}{c}93,1 \\
38\end{array}$ & 91,5 & 93,4 & 92,5 & 92,3 & 92,1 \\
\hline Tous les produits & 0,3 & $\begin{array}{l}0,4 \\
0.9\end{array}$ & 0,9 & $\begin{array}{l}0,0 \\
1,0\end{array}$ & $\begin{array}{l}3,4 \\
3.3\end{array}$ & 3,5 & 3,9 & 4.0 & $\begin{array}{l}, 0.6 \\
44\end{array}$ & $\begin{array}{l}0,8 \\
45\end{array}$ & $\begin{array}{l}1,0 \\
47\end{array}$ & $\begin{array}{r}1,4 \\
45\end{array}$ & $\begin{array}{l}1,6 \\
40\end{array}$ & $\begin{array}{r}1,5 \\
26\end{array}$ & $\begin{array}{r}1,0 \\
34\end{array}$ & $\begin{array}{l}\text {, }, 4 \\
37\end{array}$ & 3,8 & $\begin{array}{r}4,6 \\
3,9\end{array}$ & 3,7 & 1,8 & 1,4 & $\begin{array}{l}1,8 \\
29\end{array}$ \\
\hline Production facultative & & & & & 0,1 & $\begin{array}{l}3,5 \\
0,0\end{array}$ & 0,0 & 0,0 & & & & 0,0 & 0,0 & 0,0 & 0,0 & 0,0 & 0,0 & 0,0 & 0,1 & $\begin{array}{l}3,1 \\
2.6\end{array}$ & $\begin{array}{l}3,2 \\
3,1\end{array}$ & $\begin{array}{l}2,9 \\
3,1\end{array}$ \\
\hline \multicolumn{23}{|c|}{$\begin{array}{l}\text { Propodction tacutative } \\
\text { Proportion chaque label } \\
\text { Avec linites }\end{array}$} \\
\hline Avec limites & & & & & 0,0 & & & & & & & & & & & & & 0,5 & 0,4 & 2,8 & 3,1 & 3,1 \\
\hline Sans limites & 100,0 & 100,0 & 100,0 & 100,0 & 100,0 & 100,0 & 100,0 & 100,0 & 100,0 & 100,0 & 100,0 & 100,0 & 100,0 & 100,0 & 100,0 & 100,0 & 100,0 & 99,5 & 99,6 & 97,2 & 96,9 & 96,9 \\
\hline Fixe & 0,5 & 0,9 & 1,0 & 1,4 & 3,7 & 4,0 & 4,5 & 4,7 & 4,7 & 5,1 & 5,6 & 5,8 & 5,3 & 3,6 & 4,1 & 5,9 & 6,3 & 8,0 & 6,4 & 11,1 & 9,6 & 9,0 \\
\hline $\begin{array}{l}\text { Variable } \\
\text { Avec c. (obligatoire) }\end{array}$ & 99,5 & 99,1 & 99,0 & 98,6 & 96,2 & 96,0 & 95,5 & 95,3 & 95,3 & 94,9 & 94,4 & 94,2 & 94,7 & 96,4 & 95,9 & 94,1 & 93,7 & 91,9 & 93,6 & 88,9 & 90,4 & 91,0 \\
\hline $\begin{array}{l}\text { Avec c. (obligatiore) } \\
\text { Avec c. (volontaire) }\end{array}$ & & 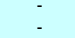 & - & - & $\begin{array}{l}0,0 \\
0,1\end{array}$ & $\begin{array}{l}0,1 \\
0,0\end{array}$ & $\begin{array}{l}0,1 \\
0,0\end{array}$ & $\begin{array}{l}0,1 \\
0,0\end{array}$ & 0,1 & 0,3 & 0,3 & $\begin{array}{l}0,4 \\
0,0\end{array}$ & $\begin{array}{l}0,3 \\
0,0\end{array}$ & $\begin{array}{l}0,4 \\
0,0\end{array}$ & $\begin{array}{l}0,2 \\
0,0\end{array}$ & $\begin{array}{l}1,1 \\
0,0\end{array}$ & $\begin{array}{l}1,9 \\
0,0\end{array}$ & $\begin{array}{l}2,4 \\
0,0\end{array}$ & $\begin{array}{l}2,2 \\
0,1\end{array}$ & $\begin{array}{l}0,1 \\
0,0\end{array}$ & 0,2 & $\begin{array}{l}0,2 \\
0,3\end{array}$ \\
\hline Sans contrair & 0,5 & 1,2 & 1,1 & 1,5 & 3,8 & 3,9 & 4,5 & 4,7 & 5,1 & 5,0 & 5,4 & 5,4 & 5,3 & 3,7 & 4,2 & 5,0 & 5,0 & 6,1 & 4,4 & 11,1 & 9,5 & 8,9 \\
\hline Un seul prod & 99,5 & 98,8 & 98,9 & 98,5 & 96,2 & 96,0 & 95,4 & 95,2 & 94,8 & 94,7 & 94,3 & 94,1 & 94,4 & 95,9 & 95,6 & 94,0 & 93,1 & 91,5 & 93,4 & 92,5 & 92,3 & 92,1 \\
\hline Un groupe de produits & 0,2 & 0,4 & 0,2 & 0,5 & 0,4 & 0,5 & 0,7 & 0,8 & 0,8 & 0,8 & 1,0 & 1,4 & 1,6 & 1,5 & 1,0 & 2,4 & 3,8 & 4,6 & 3,7 & 1,8 & 1,4 & 1,8 \\
\hline $\begin{array}{l}\text { Tous les produits } \\
\text { Superficie }\end{array}$ & 0,3 & 0,9 & 0,9 & 1,0 & 3,3 & 3,5 & 3,9 & 4,0 & 4,4 & 4,5 & 4,7 & 4,5 & 4,0 & 2,6 & 3,4 & 3,7 & $\begin{array}{l}3,1 \\
1,8\end{array}$ & $\begin{array}{l}3,9 \\
27\end{array}$ & 2,8 & 3,1 & 3,2 & 2,9 \\
\hline $\begin{array}{l}\text { Superficie } \\
\text { Nbre d'animaux }\end{array}$ & & & 0,0 & & 0.0 & & & 0,0 & 0,1 & 0.1 & 0.0 & 0,1 & 0.1 & 0,0 & $0,0,0$ & $\begin{array}{l}1,0 \\
0,3\end{array}$ & $\begin{array}{l}1,8 \\
0,3\end{array}$ & $\begin{array}{l}2,7 \\
0,4\end{array}$ & $\begin{array}{l}2,4 \\
0,1\end{array}$ & $\begin{array}{l}6,6 \\
0,1\end{array}$ & $\begin{array}{l}5,1 \\
0,1\end{array}$ & $\begin{array}{l}4,4 \\
0,0\end{array}$ \\
\hline Recettes & 0,0 & 0,3 & 0,2 & 0,1 & 0 , & 0,0 & 0,1 & 0 , & 0,5 & 0,2 & 0,1 & 0,1 & & 0,5 & 0,3 & 0,1 & 0,6 & 0,5 & 0,2 & 0,1 & 0,0 & 0,1 \\
\hline Revenu & 0,2 & 0,2 & 0,1 & 0,1 & 1,8 & 1,9 & 2,0 & 1,5 & 1,2 & 1,0 & 1,1 & 0,8 & 0,5 & 0,4 & 1,3 & 2,0 & 1,3 & 2,4 & 1,8 & 2,2 & 2,2 & 1,9 \\
\hline $\begin{array}{l}\text { Avec exceptions } \\
\text { Sans exceptions }\end{array}$ & & & & & & & & & & & & & & & & & & & 0.0 & 2,5 & 3,1 & 3.1 \\
\hline
\end{tabular}


Tableau annexe 16. Mexique : composition de l'ESP par catégorie et étiquette, 1986-2007

Part en pourcentage de l'ESP

\begin{tabular}{|c|c|c|c|c|c|c|c|c|c|c|c|c|c|c|c|c|c|c|c|c|c|c|}
\hline$\overline{\text { PSE (millions MXN) }}$ & $\begin{array}{l}1986 \\
322\end{array}$ & $\frac{1987}{1429}$ & $\begin{aligned} 1988 \\
(778)\end{aligned}$ & $\frac{1989}{6339}$ & $\begin{array}{l}1990 \\
12319\end{array}$ & $\begin{array}{l}1991 \\
22383\end{array}$ & $\frac{1992}{25174}$ & $\begin{array}{l}1993 \\
29761\end{array}$ & $\begin{array}{l}1994 \\
23900\end{array}$ & 1995 & $\frac{1996}{11273}$ & $\begin{array}{l}1997 \\
33794\end{array}$ & 1998 & 1999 & 2000 & 2001 & 2002 & 2003 & 2004 & 2005 & 2006 & $\begin{array}{l}2007 \\
66149\end{array}$ \\
\hline \multirow{2}{*}{\multicolumn{23}{|c|}{ Composition par catégorie avec labels correspondants }} \\
\hline & & & & & & & & & & & & & & & & & & & & & & \\
\hline $\begin{array}{l}\text { Production } \\
\text { Intrants }\end{array}$ & $\begin{array}{l}(175,9) \\
275,9\end{array}$ & $\begin{array}{c}(39,3) \\
139,3\end{array}$ & $\begin{array}{l}497,4 \\
(397,1)\end{array}$ & 38,0 & 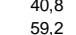 & $\begin{array}{l}81,5 \\
18,5\end{array}$ & 85,1 & $\begin{aligned} 81,6 \\
183,\end{aligned}$ & 55,7 & $\begin{array}{l}250,1 \\
684\end{array}$ & $(28,8)$ & 62,6 & $\begin{array}{c}71,3 \\
89\end{array}$ & 67,2 & $\begin{array}{l}72,7 \\
10.5\end{array}$ & 63,7 & 74,9 & 55,0 & $\begin{array}{l}34,0 \\
316\end{array}$ & $\begin{array}{ll}36,7 \\
35,2\end{array}$ & 43,0 & 30,1 \\
\hline $\mathrm{S} / \mathrm{Na} / \mathrm{Rc} / \mathrm{Rv}$ & & (159, & $\begin{aligned}(30,1) \\
(0,2)\end{aligned}$ & $\begin{array}{r}0,9 \\
0,1\end{array}$ & $\begin{array}{c}39,2 \\
0,1\end{array}$ & $\begin{array}{r}10,0 \\
0,0\end{array}$ & $\begin{array}{r}14,9 \\
0,1\end{array}$ & $\begin{array}{r}10,3 \\
0,0\end{array}$ & 20,3 & $\begin{array}{l}(0,8), 7) \\
(81,7)\end{array}$ & $\begin{array}{l}61,2 \\
61,2\end{array}$ & $\begin{array}{l}13,7 \\
23,7\end{array}$ & $\begin{array}{r}0,9 \\
19,8\end{array}$ & 20,5 & $\begin{array}{l}10,8 \\
16,8\end{array}$ & $\begin{array}{l}10,4 \\
19,9\end{array}$ & $\begin{array}{l}10,2 \\
14,2\end{array}$ & 22,4 & $\begin{array}{l}51,0 \\
33,9\end{array}$ & $\begin{array}{l}30,2 \\
28,2\end{array}$ & $\begin{array}{l}33,0 \\
23,8\end{array}$ & $\begin{array}{l}40,9 \\
28,9\end{array}$ \\
\hline Superficie & - & - & $(0,2)$ & 0,1 & 0,1 & 0,0 & 0,1 & 0,0 & 20,3 & $(81,7)$ & 61,2 & 22,8 & 19,0 & 19,2 & 16,0 & 19,0 & 13,6 & 20,0 & 30,8 & 24,4 & 20,7 & 22,7 \\
\hline $\begin{array}{l}\text { Nbre d'animaux } \\
\text { Recettes }\end{array}$ & - & - & $(0,0)$ & & & & & & & $(0,0)$ & & & & & & & & 2,1 & 3,1 & 3,7 & 3,1 & \\
\hline $\begin{array}{l}\text { Recentes } \\
\text { Revenu }\end{array}$ & - & : & $\begin{array}{l}(0,0) \\
(0,0)\end{array}$ & - & - & : & - & & & $\begin{array}{l}(0,0) \\
(0,0)\end{array}$ & . & 0,9 & 0,8 & 1,3 & 0,8 & 1,0 & 0,6 & 0,3 & & & & \\
\hline Autres produits & & & $(0,0)$ & & & & & & & $(0,0)$ & & & & & & & & 0,6 & 0,5 & & 0,2 & \\
\hline Courant & 100,0 & 100,0 & 100,0 & 100,0 & 100,0 & 100,0 & 100,0 & 100,0 & 79,7 & 180,7 & 40,2 & 77,7 & 82,2 & 81,4 & 84,6 & 82,0 & 87,1 & 79,5 & 68,5 & 73,2 & 78,5 & 75,1 \\
\hline Non-courant & & & $(0,0)$ & & & & & & 20,3 & $(80,7)$ & 59,8 & 22,3 & 17,8 & 18,6 & 15,4 & 18,0 & 12,9 & 20,5 & 31,5 & 26,8 & 21,5 & 24,9 \\
\hline Courant/variable & $(73,9)$ & 2,1 & 370,1 & 52,3 & 50,7 & 86,7 & 90,3 & 89,1 & 62,4 & 231,1 & $(14,3)$ & $\begin{array}{l}65,0 \\
1,7\end{array}$ & 73,3 & 70,7 & 75,3 & 69,7 & 79,6 & 64,7 & 49,0 & 49,6 & 56,7 & 44,1 \\
\hline $\begin{array}{l}\text { Courantifixe } \\
\text { Non-courant/variable }\end{array}$ & $\begin{array}{r}173,9 \\
\end{array}$ & 97,9 & $\begin{array}{l}(270,1) \\
(0,0)\end{array}$ & 47,7 & 49,3 & 13,3 & 9,7 & 10,9 & 17,3 & $\begin{array}{r}(50,5) \\
(0,0)\end{array}$ & 54,4 & 12,7 & 8,9 & 10,7 & 9,3 & 12,2 & 7,5 & 14,2 & 19,1 & 23,6 & 21,6 & \\
\hline $\begin{array}{l}\text { Non-courant fixe } \\
\text { Autres produits }\end{array}$ & & - & $\begin{array}{l}(0,0) \\
(0,0)\end{array}$ & - & & - & - & - & 20,3 & $\begin{array}{r}(80,7) \\
(0,0)\end{array}$ & 59,8 & 22,3 & 17,8 & 18,6 & 15,4 & 18,0 & 12,9 & $\begin{array}{r}20,5 \\
0,6\end{array}$ & $\begin{array}{r}31,5 \\
0,5\end{array}$ & 26,8 & $\begin{array}{r}21,5 \\
0,2\end{array}$ & 24,9 \\
\hline Production requise & 100,0 & 100,0 & $\begin{array}{l}100,0 \\
100,0\end{array}$ & 100,0 & 100,0 & 100,0 & 100,0 & 100,0 & 79,7 & $\begin{array}{l}180,7 \\
180,7\end{array}$ & 40,2 & 77,7 & 82,2 & 81,4 & & 82,0 & & 81,0 & 71,1 & 76,9 & $\frac{0,4}{81,4}$ & \\
\hline Un seul produit & $(172,0)$ & $(38,3)$ & & 40,1 & & 82,4 & 85,2 & 85,3 & 56,2 & 248,3 & $(24,1)$ & 63,7 & 73.5 & 70,9 & 75,5 & 64,6 & 75,3 & 57,6 & 37,6 & 41,5 & 47,6 & $\begin{array}{l}42,0 \\
42,0\end{array}$ \\
\hline Un groupe de produits & 138,7 & 87,2 & $(230,2)$ & 26,8 & 33,4 & 11,5 & 7,5 & 8,6 & 13,1 & $(37,1)$ & 41,9 & 6,1 & 5,0 & 5,1 & 4,0 & 6,5 & 5,6 & 6,8 & 9,7 & 9,4 & 8,6 & 11,5 \\
\hline Tous les produits & 133,3 & 51,1 & $(164,5)$ & 33,0 & 24,9 & 6,2 & 7,4 & 6,2 & 10,4 & $(30,5)$ & 22,4 & 7,9 & 3,7 & 5,4 & 5,1 & 10,9 & 6,2 & 16,5 & 23,8 & 26,0 & 25,2 & 27,8 \\
\hline \multirow{2}{*}{\multicolumn{23}{|c|}{$\begin{array}{l}\text { Production facultative } \\
\text { Proportion de chaque label }\end{array}$}} \\
\hline & & & & & & & & & & & & & & & & & & & & & & \\
\hline $\begin{array}{l}\text { Avec limites } \\
\text { Sans limites }\end{array}$ & $\begin{array}{r}0,4 \\
99,6\end{array}$ & $\begin{array}{l}0,1 \\
99,9\end{array}$ & $\begin{array}{c}(0,5) \\
1005\end{array}$ & $\begin{array}{r}0,2 \\
998\end{array}$ & $\begin{array}{r}0,4 \\
99,6\end{array}$ & $\begin{array}{r}0,5 \\
99,5\end{array}$ & $\begin{array}{r}0,5 \\
99,5\end{array}$ & $\begin{array}{r}0,6 \\
99,4\end{array}$ & $\begin{array}{l}21,1 \\
78,9\end{array}$ & $\begin{array}{l}(84,0) \\
1840\end{array}$ & $\begin{array}{l}61,1 \\
38,9\end{array}$ & $\begin{array}{l}22,8 \\
772\end{array}$ & $\begin{array}{l}19,0 \\
810\end{array}$ & $\begin{array}{l}19,2 \\
80,8\end{array}$ & $\begin{array}{l}16,0 \\
840\end{array}$ & $\begin{array}{l}24,3 \\
75,7\end{array}$ & $\begin{array}{l}17,7 \\
82.3\end{array}$ & $\begin{array}{l}26,9 \\
73,1\end{array}$ & $\begin{array}{l}41,5 \\
58,5\end{array}$ & 35,1 & 30,6 & $\begin{array}{l}32,8 \\
67,2\end{array}$ \\
\hline $\begin{array}{l}\text { Sans limites } \\
\text { Fixe }\end{array}$ & $\begin{array}{r}99,6 \\
173,9\end{array}$ & $\begin{array}{l}99,9 \\
97,9\end{array}$ & $\begin{array}{l}100,5 \\
(270,1)\end{array}$ & $\begin{array}{l}9,8 \\
4,7\end{array}$ & & $\begin{array}{l}99,5 \\
13,3\end{array}$ & $\begin{array}{r}99,5 \\
9,7\end{array}$ & $\begin{array}{l}99,4 \\
10,9\end{array}$ & $\begin{array}{r}8,9 \\
37,6\end{array}$ & $\begin{array}{l}184,0 \\
(131,1)\end{array}$ & $\begin{array}{r}38,9 \\
114,3\end{array}$ & $\begin{array}{l}77,2 \\
35,0\end{array}$ & & $\begin{array}{l}80 \\
29\end{array}-x-10$ & & & & & & $\begin{array}{l}64,9 \\
50,4\end{array}$ & $\begin{array}{l}69,4 \\
43,1\end{array}$ & $\begin{array}{l}67,2 \\
55,9\end{array}$ \\
\hline $\begin{array}{l}\text { Fixe } \\
\text { Variable }\end{array}$ & $\begin{array}{l}(73,9 \\
(73,9)\end{array}$ & $\begin{array}{r}\begin{array}{r}97,9 \\
2,1\end{array} \\
\end{array}$ & $\begin{array}{l}(270,1) \\
370,1\end{array}$ & $\begin{array}{l}4,7 \\
52,3\end{array}$ & $\begin{array}{l}49,3 \\
50,7\end{array}$ & $\begin{array}{l}13,3 \\
86,7\end{array}$ & $\begin{array}{r}9,7 \\
90,3\end{array}$ & $\begin{array}{l}89,9 \\
89,1\end{array}$ & $\begin{array}{l}37,6 \\
62,4\end{array}$ & $\begin{array}{l}(131,1) \\
231,1\end{array}$ & $\begin{array}{l}114,3 \\
(14,3)\end{array}$ & $\begin{array}{l}35,0 \\
65,0\end{array}$ & $\begin{array}{l}20,1 \\
73,3\end{array}$ & $\begin{array}{l}\begin{array}{l}29,3 \\
70,7\end{array} \\
-\end{array}$ & $\begin{array}{l}\begin{array}{l}24,7 \\
75,3\end{array} \\
-\end{array}$ & $\begin{array}{l}69,7 \\
69,7\end{array}$ & $\begin{array}{l}20,4 \\
79,6\end{array}$ & $\begin{array}{l}34,6 \\
64,7\end{array}$ & $\begin{array}{r}40,5 \\
49,0\end{array}$ & $\begin{array}{l}50,4 \\
49,6\end{array}$ & $\begin{array}{l}43,1 \\
56,7\end{array}$ & $\begin{array}{l}55,9 \\
44,1\end{array}$ \\
\hline Avec c. (obligatoire) & & & $(0,0)$ & & & & & & & $(0,0)$ & & & & & & & & & & & & \\
\hline $\begin{array}{l}\text { Avec c. (volontaire) } \\
\text { Sans contrainte }\end{array}$ & 272 & 1394 & $\begin{aligned} & 10-1 \\
& 1397\end{aligned}$ & 621 & 596 & & $\begin{array}{r}0,0 \\
155\end{array}$ & 0,0 & 4 & & & 375 & 288 & 329 & & & & $\begin{array}{r}2,8 \\
472\end{array}$ & 3,6 & 3,7 & 3,3 & $\begin{array}{r}6,2 \\
66,4\end{array}$ \\
\hline $\begin{array}{l}\text { Sans contrante } \\
\text { Un seul produit }\end{array}$ & $\begin{array}{l}216,2,2 \\
(172,0)\end{array}$ & $\begin{array}{l}3199,4 \\
(38,3)\end{array}$ & $\begin{array}{r}39 \\
49 \\
-19\end{array}$ & $\begin{array}{l}2,1 \\
40,1\end{array}$ & $\begin{array}{l}59,6 \\
41,7\end{array}$ & $\begin{array}{l}91,0 \\
82,4\end{array}$ & $\begin{array}{l}15,5 \\
85,2\end{array}$ & $\begin{array}{l}19,1 \\
85,3\end{array}$ & $\begin{array}{l}45,2 \\
56,2\end{array}$ & $\begin{array}{l}(152,7) \\
248,3\end{array}$ & $\begin{array}{l}\begin{array}{r}29,0 \\
(24,1)\end{array} \\
-\end{array}$ & $\begin{array}{l}37,5 \\
63,7\end{array}$ & $\begin{array}{l}28,8 \\
73,5\end{array}$ & $\begin{array}{l}32,9 \\
70,9\end{array}$ & $\begin{array}{l}27,4 \\
75,5\end{array}$ & $\begin{array}{l}43,2 \\
64,6\end{array}$ & $\begin{array}{l}30,2 \\
75,3\end{array}$ & $\begin{array}{l}41,2 \\
57,6\end{array}$ & $\begin{array}{l}68,1 \\
37,6\end{array}$ & $\begin{array}{l}68,2 \\
41,5\end{array}$ & $\begin{array}{l}60,4 \\
47,6\end{array}$ & $\begin{array}{l}66,4 \\
42,0\end{array}$ \\
\hline Un groupe de produits & 138,7 & 87,2 & $(230,2)$ & 26,8 & 33 & 11,5 & 7,5 & 8,6 & 13,1 & & 41,9 & 6,1 & 5,0 & 5,1 & 4,0 & 6,5 & 5,6 & & 9,7 & 9,4 & 8,6 & 11,5 \\
\hline Tous les produits & 133,3 & 51,1 & & 33,0 & 24,9 & 6,2 & 7,4 & 6,2 & & & 22,4 & 7,9 & & 5,4 & 5,1 & 10,9 & 6,2 & & & & 25,2 & 27,8 \\
\hline $\begin{array}{l}\text { Superficie } \\
\text { Nbre d'animaux }\end{array}$ & - & . & $\begin{array}{l}(0,2) \\
(0,0)\end{array}$ & 0,1 & 0,1 & 0,0 & 0,1 & 0,0 & 20,3 & $\begin{array}{c}(81,7) \\
(0,0)\end{array}$ & 61,2 & 22,8 & 19,0 & 19,2 & 16,0 & 19,0 & 13,6 & $\begin{array}{r}20,0 \\
2,1\end{array}$ & $\begin{array}{r}30,8 \\
3,1\end{array}$ & $\begin{array}{r}24,4 \\
3,7\end{array}$ & $\begin{aligned} 20,7 \\
3,1\end{aligned}$ & $\begin{aligned} 22,7 \\
6,2\end{aligned}$ \\
\hline & - & - & $(0,0)$ & - & - & - & - & - & 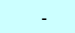 & & - & & & & & & & & & & & \\
\hline & & - & $(0,0)$ & & & 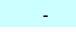 & - & & & o) & & 0,9 & 0,8 & & 0,8 & 1,0 & 0,6 & 0,3 & & & & \\
\hline $\begin{array}{l}\text { Avec excepptions } \\
\text { Sans exceptions }\end{array}$ & & $\therefore$ & $\begin{array}{c}(0,0) \\
(0,0)\end{array}$ & & & & & & 20,3 & $\begin{array}{c}(80,7) \\
(0,0)\end{array}$ & 59,8 & 22,3 & 17,8 & 18,6 & 15,4 & 18,0 & 12,9 & 18,4 & 28,4 & 23,1 & 18,4 & 18,6 \\
\hline
\end{tabular}


Tableau annexe 17. Nouvelle-Zélande : composition de l'ESP par catégorie et étiquette, 1986-2007

Part en pourcentage de l'ESP

\begin{tabular}{|c|c|c|c|c|c|c|c|c|c|c|c|c|c|c|c|c|c|c|c|c|c|c|}
\hline & 1986 & 1987 & 1988 & 1989 & 1990 & 1991 & 1992 & 1993 & 1994 & 1995 & 1996 & 1997 & 1998 & 1999 & 2000 & 2001 & 2002 & 2003 & 2004 & 2005 & 2006 & 2007 \\
\hline PSE (millions NZD) & 1511 & 566 & 265 & 179 & 159 & 131 & 92 & 67 & 116 & 142 & 99 & 112 & 101 & 101 & 62 & 114 & 60 & 125 & 119 & 196 & 148 & 112 \\
\hline \multicolumn{23}{|c|}{ Composition par catégorie avec labels correspondants } \\
\hline Production & 9,1 & 20,9 & 27,4 & 38,0 & 40,5 & 57,0 & 50,7 & 47,2 & 49,9 & 58,9 & 42,9 & 48,8 & 43,4 & 44,0 & 1,5 & 36,7 & 15,9 & 57,8 & 44,9 & 50,8 & 56,8 & \\
\hline 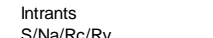 & 26,4 & 75,9 & 43,0 & 45,4 & 42,0 & 30,3 & 43,5 & 51,0 & 49,9 & 41,1 & 55,8 & 50,8 & 56,1 & 55,9 & 90,4 & 63,1 & 84,1 & 42,2 & 50,3 & 27,3 & 37,7 & 54,0 \\
\hline $\begin{array}{l}\text { S/Na/Re/Rivi } \\
\text { Supericie }\end{array}$ & 64,5 & 3,3 & 29,5 & 16,6 & 17,5 & 12,7 & 5,8 & 1,8 & 0,1 & & 1,3 & 0,4 & 0,5 & 0,1 & 8,0 & 0,2 & & 0,0 & 4,8 & 22,0 & 5,5 & 0,5 \\
\hline Nbre d'animaux & 62,5 & - & - & - & - & - & - & - & - & - & - & - & - & - & - & - & - & - & - & - & - & \\
\hline Revenu & 2,0 & 3,3 & 29,5 & 16,6 & 17,5 & 12,7 & 5,8 & 1,8 & 0,1 & - & 1,3 & 0,4 & 0,5 & 0,1 & 8,0 & 0,2 & - & 0,0 & 4,8 & 22,0 & 5,5 & 0,5 \\
\hline $\begin{array}{l}\text { Autres produits } \\
\text { Courant }\end{array}$ & 37,5 & 1000 & 1000 & 100.0 & 11000 & 1000 & 1000 & 1000 & 1000 & 100,0 & 1100 & 1000 & 1000 & 1000 & 1000 & 1000 & 1000 & 1000 & 1000 & 1000 & 1000 & 100,0 \\
\hline Non-courant & $\begin{array}{l}62,5 \\
6,25\end{array}$ & & & & & & & & & 100,0 & & & & & & & & & & & & \\
\hline Courant/variable & 9,0 & 21,1 & 51,5 & 48,1 & 55,9 & 69,7 & 56,5 & 49,0 & 50,1 & 58,9 & 44,2 & 49,2 & 43,9 & & 9,6 & 36,9 & 15,9 & 57,8 & 49,7 & 72,7 & 62,3 & 46,0 \\
\hline $\begin{array}{l}\text { Courantffixe } \\
\text { Non-courant/variable }\end{array}$ & 28,5 & 78,9 & 48,5 & 51,9 & 44,1 & 30,3 & 43,5 & 51,0 & 49,9 & 41,1 & 55,8 & 50,8 & 56,1 & 55,9 & 90,4 & 63,1 & 84,1 & 42,2 & 50,3 & 27,3 & 37,7 & 54,0 \\
\hline Non-courant/fixe & 62,5 & - & - & - & - & - & - & - & - & - & - & - & - & - & - & - & - & - & - & - & - & - \\
\hline $\begin{array}{l}\text { Autres probulits } \\
\text { Production requise }\end{array}$ & 1000 & 1000 & 1000 & 1000 & 1000 & 1000 & 1000 & 1000 & 1000 & 1000 & 100,0 & 1000 & 1000 & 1000 & 1000 & 1000 & 1000 & 1000 & 1000 & 1000 & 1000 & 1000 \\
\hline Un seul produit & 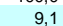 & 20,9 & 27.4 & 38,0 & 40,5 & 57,0 & 50,7 & 47,2 & 49,9 & 58.9 & 42,9 & 48.8 & 43,4 & 44,0 & $\begin{array}{l}1,5 \\
1.5\end{array}$ & $\begin{array}{l}36,7 \\
\end{array}$ & 15,9 & 57.8 & 44,9 & 50.8 & 56,8 & 45,5 \\
\hline Un groupe de produits & 88,2 & 74,4 & 40,8 & 43,6 & 38,6 & 27,8 & 41,7 & 47,9 & 28,6 & 26,9 & 34,1 & 30,5 & 33,7 & 36,4 & 61,5 & 43,6 & 48,2 & 25,7 & 27,1 & 16,4 & 21,7 & 28,7 \\
\hline Tous les produits & 2,7 & 4,7 & 31,8 & 18,4 & 20,9 & 15,3 & 7,6 & 4,8 & 21,5 & 14,2 & 22,9 & 20,8 & 23,0 & 19,6 & 36,9 & 19,6 & 35,9 & 16,5 & 28,0 & 32,9 & 21,5 & 25,8 \\
\hline \multirow{2}{*}{\multicolumn{23}{|c|}{ Proportion de chaque label }} \\
\hline Avec limites & & & & & & & & & & & & & & & & & & & & & & \\
\hline Sans limites & 99,5 & 100,0 & 100,0 & 100,0 & 100,0 & 100,0 & 100,0 & 100,0 & 100,0 & 100,0 & 100,0 & 100,0 & 100,0 & 100,0 & 100,0 & 100,0 & 100,0 & 100,0 & 100,0 & 100,0 & 100,0 & 100,0 \\
\hline Fixe & 91,0 & 78,9 & 48,5 & 51,9 & 44,1 & 30,3 & 43,5 & 51,0 & 49,9 & 41,1 & 55,8 & 50,8 & 56,1 & 55,9 & 90,4 & 63,1 & 84,1 & 42,2 & 50,3 & 27,3 & 37,7 & 54,0 \\
\hline Variable & 9,0 & 21,1 & 51,5 & 48,1 & 55,9 & 69,7 & 56,5 & 49,0 & 50,1 & 58,9 & 44,2 & 49,2 & 43,9 & 44,1 & 9,6 & 36,9 & 15,9 & 57,8 & 49,7 & 72,7 & 62,3 & 46,0 \\
\hline $\begin{array}{l}\text { Avec c. (obligatioire) } \\
\text { Avec c. (volontair) }\end{array}$ & . & 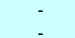 & 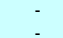 & 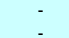 & 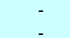 & 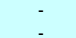 & 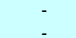 & & - & - & & & & & & & & & & & & \\
\hline $\begin{array}{l}\text { Avec c. (uolontaire) } \\
\text { Sans contrainte }\end{array}$ & 91.4 & 79.1 & 726 & 620 & 59.5 & 430 & 493 & 528 & 501 & 411 & 571 & 512 & 566 & 560 & 085 & 633 & 841 & 422 & & & 432 & 54,5 \\
\hline $\begin{array}{l}\text { Sans contrante } \\
\text { Un seul produit }\end{array}$ & 91,4 & $\begin{array}{l}9,1 \\
20,9\end{array}$ & $\begin{array}{l}27,0 \\
27,4\end{array}$ & $\begin{array}{l}0<, 0 \\
380\end{array}$ & 405 & $\begin{array}{l}43,0 \\
570\end{array}$ & $\begin{array}{l}49,3 \\
507\end{array}$ & $\begin{array}{l}52,8 \\
47,2\end{array}$ & 50,1 & $\begin{array}{l}41,1 \\
589\end{array}$ & $\begin{array}{l}5 /, 1 \\
429\end{array}$ & $\begin{array}{l}51,2 \\
488\end{array}$ & $\begin{array}{l}56,6 \\
434\end{array}$ & 56,0 & $\begin{array}{l}98,5 \\
15\end{array}$ & $\begin{array}{l}63,3 \\
367\end{array}$ & $\begin{array}{l}84,1 \\
159\end{array}$ & $\begin{array}{l}42,2 \\
578\end{array}$ & 55,1 & $\begin{array}{l}49,2 \\
508\end{array}$ & $\begin{array}{l}43,2 \\
5,2\end{array}$ & $\begin{array}{l}54,5 \\
45,5\end{array}$ \\
\hline Un groupe & $\begin{array}{l}9,1 \\
88.2\end{array}$ & $\begin{array}{l}74,4 \\
74.4\end{array}$ & 40 & $\begin{array}{l}38,0 \\
43,6\end{array}$ & $\begin{array}{l}38,6 \\
38,6\end{array}$ & $\begin{array}{l}57,0 \\
27,8\end{array}$ & 41,7 & 47.9 & $\begin{array}{l}4,9,9 \\
28,6\end{array}$ & $\begin{array}{l}50,9 \\
26,9\end{array}$ & $\begin{array}{l}4<, 9 \\
34,1\end{array}$ & $\begin{array}{l}40,6 \\
30,5\end{array}$ & 337 & $\begin{array}{l}36,4 \\
36.4\end{array}$ & $\begin{array}{l}6,5 \\
615\end{array}$ & 436 & 482 & 257 & 277 & $\begin{array}{l}5,6 \\
164\end{array}$ & 117 & $\begin{array}{l}40,5 \\
28,7\end{array}$ \\
\hline & 2,7 & 4,7 & 31,8 & 18,4 & 20,9 & $\begin{array}{l}15,3 \\
15,3\end{array}$ & 7,6 & 4,8 & 21,5 & 14,2 & 22,9 & 20,8 & 23,0 & 19,6 & 36,9 & 19,6 & 35,9 & 16,5 & 28,0 & 32,9 & 21,5 & 25,8 \\
\hline & & & & & & & & & & & & & & & & & & & & & & \\
\hline $\begin{array}{l}\text { Nbre d'animaux } \\
\text { Recettes }\end{array}$ & 62,5 & $\cdot$ & $\cdot$ & $\cdot$ & $\cdot$ & - & - & - & $\cdot$ & - & $\cdot$ & - & - & . & $\cdot$ & $\cdot$ & : & $\cdot$ & $\cdot$ & $\cdot$ & - & - \\
\hline Revenu & 2,0 & 3,3 & 29,5 & 16,6 & 17,5 & 12,7 & 5,8 & 1,8 & 0,1 & . & 1,3 & 0,4 & 0,5 & 0,1 & 8,0 & 0,2 & . & 0,0 & 4,8 & 22,0 & 5,5 & 0,5 \\
\hline $\begin{array}{l}\text { Avec exceptions } \\
\text { Sans sexcentions }\end{array}$ & & & & & & & & & & & & & & & & & & & & & & \\
\hline
\end{tabular}


Tableau annexe 18. Norvège : composition de l'ESP par catégorie et étiquette, 1986-2007

Part en pourcentage de l'ESP

\begin{tabular}{|c|c|c|c|c|c|c|c|c|c|c|c|c|c|c|c|c|c|c|c|c|c|c|}
\hline & 1986 & 1987 & 1988 & 1989 & 1990 & 1991 & 1992 & 1993 & 1994 & 1995 & 1996 & 1997 & 1998 & 1999 & 2000 & 2001 & 2002 & 2003 & 2004 & 2005 & 2006 & 2007 \\
\hline PSE (millions NOK) & 18333 & 20093 & 19145 & 19402 & 22084 & 22985 & 21307 & 21552 & 20650 & 18912 & 19443 & 19990 & 21229 & 20595 & 18744 & 19130 & 22328 & 21350 & 20008 & 19647 & 19288 & 16422 \\
\hline \multicolumn{23}{|c|}{ Composition par catégorie avec labels correspondants } \\
\hline Production & 72,9 & 73,3 & 70,9 & 71,1 & 75,1 & 71,5 & 65,9 & 65,2 & 64,2 & 62,4 & 63,6 & 62,2 & 62,1 & 60,9 & 54,7 & 56,1 & 61,6 & 55,5 & 52,6 & 53,0 & 51,5 & \\
\hline Intrants & 9,8 & 8,8 & 8,4 & 8,1 & 5,0 & 4,4 & 4,3 & 4,0 & 3,9 & 4,3 & 4,9 & 5,5 & 5,2 & 5,5 & 6,1 & 6,0 & 4,5 & 4,6 & 5,0 & 5,5 & 5,6 & 6,8 \\
\hline $\mathrm{S} / \mathrm{Na} / \mathrm{Rc} / \mathrm{Rv}$ & 17,3 & 17,9 & 20,6 & 20,8 & 19,8 & 24,0 & 29,7 & 30,8 & 31,8 & 33,1 & 31,1 & 32,3 & 32,7 & 33,6 & 39,2 & 38,0 & 33,9 & 39,9 & 42,4 & 41,5 & 42,9 & 51,6 \\
\hline Superficie & 4,4 & 5,2 & 5,6 & 5,7 & 5,8 & 9,2 & 13,0 & 14,1 & 14,6 & 16,0 & 17,4 & 18,0 & 18,9 & 17,3 & 18,8 & 17,5 & 15,5 & 16,1 & 16,9 & 17,4 & 18,4 & 23,2 \\
\hline $\begin{array}{l}\text { Nbre d'animaux } \\
\text { Recettes }\end{array}$ & 12,9 & 12,8 & 15,0 & 15,1 & 14,1 & 14,8 & 16,7 & 16,6 & 17,1 & 17,1 & 13,8 & 14,2 & 13,8 & $\begin{array}{r}16,2 \\
0,0\end{array}$ & $\begin{array}{r}19,0 \\
0,0\end{array}$ & $\begin{array}{r}18,0 \\
0,0\end{array}$ & $\begin{array}{r}16,2 \\
0,5\end{array}$ & $\begin{array}{r}15,2 \\
5,9\end{array}$ & $\begin{array}{r}16,7 \\
6,1\end{array}$ & $\begin{array}{r}16,0 \\
5,9\end{array}$ & $\begin{array}{r}16,4 \\
5,3\end{array}$ & $\begin{array}{r}17,4 \\
5,9\end{array}$ \\
\hline Revenu & - & - & - & - & & & & & & & & & & 0,1 & 1,4 & 2,5 & 2,3 & 2,8 & 2,7 & 2,7 & 2,9 & 5,2 \\
\hline Autres produits & & & & & 0,0 & 0,0 & 0,0 & 0,1 & 0,1 & 0,2 & 0,4 & 0,0 & & & & & & & & & & \\
\hline $\begin{array}{l}\text { Courant } \\
\text { Non-courant }\end{array}$ & 100,0 & 100,0 & 100,0 & 100,0 & 100,0 & 100,0 & 100,0 & 100,0 & 100,0 & 100,0 & 100,0 & 100,0 & 100,0 & 100,0 & 100,0 & 100,0 & 100,0 & $\begin{array}{l}86,2 \\
13,8\end{array}$ & $\begin{array}{l}85,6 \\
14,4\end{array}$ & $\begin{array}{l}86,2 \\
13,8\end{array}$ & $\begin{array}{l}86,5 \\
13,5\end{array}$ & $\begin{array}{l}84,0 \\
16,0\end{array}$ \\
\hline Courant/variable & 117,1 & 107,9 & 81,8 & 80,7 & 84,6 & 75,1 & 76,0 & 71,6 & 70,9 & 57,5 & 50,8 & 48,4 & 52,9 & 55,6 & 49,8 & 53,2 & 63,6 & 57,6 & 52,8 & 52,0 & 54,8 & $\begin{array}{l}38,9 \\
38,9\end{array}$ \\
\hline 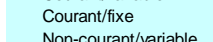 & 43,6 & 44,4 & 48,4 & 48,1 & 46,1 & 48,9 & 54,1 & 53,5 & 54,1 & 55,2 & 52,1 & 52,2 & 50,6 & 51,8 & 57,6 & 56,0 & 49,9 & 36,5 & 38,5 & 38,7 & 40,1 & 48,6 \\
\hline $\begin{array}{l}\text { Non-courantvanables } \\
\text { Non-courant/fixe }\end{array}$ & - & - & - & - & & & & & & & & & - & & . & - & - & 13,8 & 14,4 & 13,8 & 13,5 & 16,0 \\
\hline $\begin{array}{l}\text { Autres produits } \\
\text { Production requise }\end{array}$ & 1100,0 & 100,0 & 100,0 & 100,0 & $\begin{array}{r}0,0 \\
100,0\end{array}$ & $\frac{0,0}{100}$ & $\begin{array}{r}0,0 \\
1000\end{array}$ & $\begin{array}{r}0,1 \\
999\end{array}$ & $\begin{array}{r}0,1 \\
999\end{array}$ & $\begin{array}{r}0,2 \\
998 \\
98\end{array}$ & $\begin{array}{r}0,4 \\
99,6\end{array}$ & $\begin{array}{r}0,0 \\
1000\end{array}$ & & & & & & & & & & \\
\hline $\begin{array}{l}\text { Troudcition requise } \\
\text { Un seul produit }\end{array}$ & $\begin{array}{r}100,0 \\
72,9\end{array}$ & $\begin{array}{r}100,0 \\
73,3\end{array}$ & $\begin{array}{l}70,0 \\
70,9\end{array}$ & $\begin{array}{l}100,0 \\
71,1\end{array}$ & $\begin{array}{r}100,0 \\
75,1\end{array}$ & $\begin{array}{l}10,0 \\
71,5 \\
-10\end{array}$ & $\begin{array}{l}100,0 \\
659\end{array}$ & $\begin{array}{l}99,9 \\
65,2\end{array}$ & 99,9 & $\begin{array}{l}99,8 \\
62,5\end{array}$ & 93,6 & $\begin{array}{r}100,0 \\
62,3\end{array}$ & $\begin{array}{r}100,0 \\
62,1\end{array}$ & $\begin{array}{l}100,0 \\
61,0\end{array}$ & $\begin{array}{l}100,0 \\
54,8\end{array}$ & $\begin{array}{l}100,0 \\
56,2\end{array}$ & $\begin{array}{r}100,0 \\
61,6\end{array}$ & 100,0 & $\begin{array}{l}100,0 \\
57\end{array}$ & $\begin{array}{r}100,0 \\
584\end{array}$ & $\begin{array}{l}100,0 \\
567\end{array}$ & $\begin{array}{l}100,0 \\
47,4\end{array}$ \\
\hline Un groupe de produits & 22,2 & 21,9 & 24,9 & 24.9 & 21,6 & 25,3 & 30,0 & 30,8 & $\begin{array}{l}04,0 \\
31,6\end{array}$ & $\begin{array}{l}33,1 \\
33,1\end{array}$ & $\begin{array}{l}30,9 \\
31,9\end{array}$ & $\begin{array}{l}33.2 \\
33,2\end{array}$ & 33.4 & 34,0 & $\begin{array}{l}38,0 \\
38,2\end{array}$ & $\begin{array}{l}30,6 \\
36,6\end{array}$ & $\begin{array}{l}0,0 \\
31,9\end{array}$ & 23,4 & 25,7 & $\begin{array}{l}20,4 \\
253\end{array}$ & $\begin{array}{l}20,1 \\
25.9\end{array}$ & $\begin{array}{l}4,4,4 \\
29,1\end{array}$ \\
\hline Tous les produits & 4,9 & 4,8 & 4,2 & 4,0 & 3,3 & 3,1 & 4,0 & 4,0 & 3,9 & 4,2 & 4,1 & 4,5 & 4,5 & 4,9 & 6,9 & 7,2 & 6,5 & 15,2 & 15,6 & 16,3 & 17,4 & $\begin{array}{l}29,1 \\
23,4\end{array}$ \\
\hline \multirow{2}{*}{\multicolumn{23}{|c|}{$\begin{array}{l}\text { Production facultative } \\
\text { Proportion de chaque label }\end{array}$}} \\
\hline & & & & & & & & & & & & & & & & & & & & & & \\
\hline $\begin{array}{l}\text { Avec cimitis } \\
\text { Sans limites }\end{array}$ & $\begin{array}{l}30,1 \\
69,9\end{array}$ & $\begin{array}{l}31,0 \\
69,0\end{array}$ & $\begin{array}{l}33,6 \\
66,4\end{array}$ & $\begin{array}{l}32,9 \\
67,1\end{array}$ & $\begin{array}{l}37,7 \\
62.3\end{array}$ & $\begin{array}{l}35,6 \\
64,4\end{array}$ & $\begin{array}{l}38,0 \\
62,0\end{array}$ & $\begin{array}{l}36,7 \\
63,3\end{array}$ & $\begin{array}{l}37,8 \\
62,2\end{array}$ & $\begin{array}{l}33,4 \\
66,6\end{array}$ & $\begin{array}{l}35,1 \\
64,9\end{array}$ & $\begin{array}{l}34,7 \\
65,3\end{array}$ & $\begin{array}{l}33,7 \\
66,3\end{array}$ & $\begin{array}{l}34,3 \\
65,7\end{array}$ & $\begin{array}{l}33,4 \\
66,6\end{array}$ & $\begin{array}{l}31,6 \\
68,4\end{array}$ & $\begin{array}{l}34,5 \\
65,5\end{array}$ & $\begin{array}{l}32,5 \\
67,5\end{array}$ & $\begin{array}{l}31,2 \\
68,8\end{array}$ & $\begin{array}{l}30,7 \\
69,3\end{array}$ & $\begin{array}{l}29,9 \\
70,1\end{array}$ & $\begin{array}{l}20,2 \\
79,8\end{array}$ \\
\hline $\begin{array}{l}\text { Fixe } \\
\text { Finstilles }\end{array}$ & $\begin{array}{l}93,9 \\
43,6\end{array}$ & $\begin{array}{l}90,0 \\
44,4\end{array}$ & & & $\begin{array}{l}0<, 3 \\
46,1\end{array}$ & $\begin{array}{l}64,4 \\
48,9\end{array}$ & $\begin{array}{l}5<, 0 \\
54,1\end{array}$ & 53,5 & & $\begin{array}{l}\begin{array}{l}60,0 \\
55,2\end{array} \\
5\end{array}$ & $\begin{array}{l}64,9 \\
52,1\end{array}$ & $\begin{array}{l}65,5 \\
52,2\end{array}$ & $\begin{array}{l}60,5 \\
50,6\end{array}$ & $\begin{array}{l}65,1 \\
51,8\end{array}$ & $\begin{array}{l}00,0 \\
57,6\end{array}$ & & $\begin{array}{l}60,0 \\
49,9\end{array}$ & 50,2 & $\begin{array}{l}68,0 \\
52,9\end{array}$ & $\begin{array}{l}69,6 \\
52,6\end{array}$ & 53,5 & $\begin{array}{l}79,8 \\
64,7\end{array}$ \\
\hline Vari & 117,1 & 107,9 & $\begin{array}{l}80,4 \\
81,8\end{array}$ & 80,7 & 84,6 & 75,1 & 76,0 & 71,6 & 70,9 & 57,5 & 50,8 & 48,4 & $\begin{array}{l}5,0,9 \\
52,9\end{array}$ & $\begin{array}{l}5,6,6 \\
55\end{array}$ & 49,8 & $\begin{array}{l}53,2 \\
53,2\end{array}$ & $\begin{array}{l}63,6 \\
63,6\end{array}$ & $\begin{array}{l}50,6 \\
57,6\end{array}$ & 52,8 & 52,0 & 54,8 & $\begin{array}{l}64,7 \\
38,9\end{array}$ \\
\hline \multicolumn{23}{|l|}{$\begin{array}{l}\text { Varia } \\
\text { Avec }\end{array}$} \\
\hline Sans contr & 50 & 5 & 54,9 & 54 & 4 & $\begin{array}{r}0,1 \\
51,9\end{array}$ & 56,7 & 55 & $\begin{array}{r}5,8,8 \\
55\end{array}$ & $\begin{array}{r}0,0 \\
57,0\end{array}$ & 53,7 & 54,1 & $\begin{array}{r}0,0 \\
52,6\end{array}$ & $\begin{array}{l}0,1 \\
53,6\end{array}$ & $\begin{array}{r}0,9,5 \\
59\end{array}$ & & 51,0 & $\begin{array}{l}0,9 \\
43,4\end{array}$ & $\begin{array}{r}3,0 \\
45,7\end{array}$ & $\begin{array}{r}3,0 \\
45,3\end{array}$ & 45,6 & $\begin{array}{l}13,0 \\
54,4\end{array}$ \\
\hline Un & 72,9 & 73,3 & 70,9 & 71,1 & 75,1 & 71,5 & 65,9 & 65,2 & 64,3 & 62,5 & 63,7 & 62,3 & 62,1 & 61,0 & 54,8 & 56,2 & 61,6 & 61,3 & 58,7 & 58,4 & 56,7 & 47,4 \\
\hline oduits & 22,2 & 21,9 & 24,9 & 24,9 & 21,6 & & 30,0 & 30,8 & & 33,1 & 31,9 & 33,2 & 33,4 & 34,0 & 38,2 & 36,6 & 31,9 & & & 25,3 & 25,9 & 29,1 \\
\hline & 4,9 & 4,8 & 4,2 & $\begin{array}{l}4,0 \\
57\end{array}$ & 3,3 & 3,1 & 4,0 & 4,0 & 3,9 & 4,2 & 4,1 & $\begin{array}{r}4,5 \\
1,0\end{array}$ & $\begin{array}{r}4,5 \\
180\end{array}$ & $\begin{array}{r}4,9 \\
179\end{array}$ & 6,9 & 7,2 & 6,5 & 15,2 & 15,6 & 16,3 & 17,4 & 23,4 \\
\hline Superficie & 4,4 & 5,2 & $\begin{array}{r}5,6 \\
1,0\end{array}$ & $\begin{array}{r}5,7 \\
15,\end{array}$ & 5,8 & 9,2 & 13,0 & 14,1 & 14,6 & 16,0 & 17,4 & 18,0 & 18,9 & 17,3 & 18,8 & 17,5 & 15,5 & 16,1 & & 17,4 & 18,4 & 23,2 \\
\hline $\begin{array}{l}\text { Nored d'animaux } \\
\text { Recettes }\end{array}$ & 12,9 & 12,8 & 15,0 & ${ }^{15,1}$ & 14,1 & 14,8 & 16,7 & 16,6 & 17,1 & 17,1 & 13,8 & 14,2 & 13,8 & $\begin{array}{r}16,2 \\
0,0\end{array}$ & $\begin{array}{r}19,0 \\
0,0\end{array}$ & $\begin{array}{r}18,0 \\
0,0\end{array}$ & $\begin{array}{r}\begin{array}{r}6,2 \\
0,5\end{array} \\
0.5\end{array}$ & $\begin{array}{r}15,2 \\
5,9\end{array}$ & $\begin{array}{r}16,7 \\
6,1\end{array}$ & $\begin{array}{r}16,0 \\
5,9\end{array}$ & $\begin{array}{r}16,4 \\
5,3\end{array}$ & $\begin{array}{r}17,4 \\
5,9\end{array}$ \\
\hline & - & - & - & - & - & - & - & - & - & - & - & - & - & 0,1 & 1,4 & 2,5 & 2,3 & 2,8 & 2,7 & 2,7 & 2,9 & 5,2 \\
\hline $\begin{array}{l}\text { Avec exceppitions } \\
\text { Sans exceptions }\end{array}$ & & $\therefore$ & : & - & & & & & - & - & 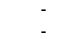 & & : & $\therefore$ & & & $\therefore$ & $\therefore$ & - & - & $\therefore$ & \\
\hline
\end{tabular}


Tableau annexe 19. Suisse : composition de l'ESP par catégorie et étiquette, 1986-2007

Part en pourcentage de l'ESP

\begin{tabular}{|c|c|c|c|c|c|c|c|c|c|c|c|c|c|c|c|c|c|c|c|c|c|c|}
\hline & 1986 & 1987 & 1988 & 1989 & 1990 & 1991 & 1992 & 1993 & 1994 & 1995 & 1996 & 1997 & 1998 & 1999 & 2000 & 2001 & 2002 & 2003 & 2004 & 2005 & 2006 & 2007 \\
\hline PSE (millions CHF) & 8189 & 8433 & 8699 & 7840 & 8261 & 8622 & 7318 & 8140 & 8130 & 7081 & 7440 & 7391 & 7498 & 7654 & 7301 & 7073 & 7647 & 7208 & 7137 & 6966 & 6115 & 5016 \\
\hline \multicolumn{23}{|c|}{ Composition par catégorie avec labels correspondants } \\
\hline Production & 83,1 & 83,2 & 83,4 & 81,1 & 84,2 & 81,3 & 78,2 & 76,6 & 74,5 & 69,7 & 66,0 & & 66,0 & 65,9 & & & & 59,5 & & & & \\
\hline Intrants & 7,0 & 7,1 & 5,9 & 5,2 & 3,6 & 3,7 & 4,2 & 5,4 & 5,9 & 6,1 & 5,3 & 5,5 & 5,1 & 2,6 & 2,9 & 3,3 & 3,3 & 3,1 & 3,1 & 3,2 & 3,7 & 5,0 \\
\hline $\mathrm{S} / \mathrm{Na} / \mathrm{Rc} / \mathrm{Rv}$ & 7,1 & 7,4 & 8,2 & 10,9 & 9,7 & 12,5 & 14,7 & 15,2 & 16,7 & 20,7 & 25,2 & 26,7 & 25,2 & 27,7 & 30,0 & 32,0 & 30,9 & 33,1 & 33,5 & 34,5 & 39,4 & 49,2 \\
\hline Superficie & 3,0 & 2,9 & 3,3 & 3,8 & 3,4 & 3,2 & 3,6 & 4,8 & 5,1 & 7,4 & 12,1 & 13,4 & 12,0 & 19,0 & 20,4 & 21,4 & 20,0 & 21,3 & 21,6 & 22,1 & 25,2 & 29,7 \\
\hline $\begin{array}{l}\text { Nore d'animaux } \\
\text { Recettes }\end{array}$ & 4,2 & 4,4 & 4,4 & 5,4 & 5,2 & 6,4 & 6,6 & 5,5 & 5,4 & 6,1 & 6,3 & 6,4 & 6,4 & 8,8 & 9,6 & 10,7 & 10,9 & 11,8 & 11,9 & 12,4 & 14,2 & \\
\hline Revenu & & & 0,5 & 1,7 & 1,1 & 2,9 & 4,5 & 4,9 & 6,3 & 7,2 & 6,9 & 6,9 & 6,8 & & & & & & & & & \\
\hline Autres produits & 2,8 & 2,4 & 2,5 & 2,7 & 2,5 & 2,5 & 3,0 & 2,8 & 2,9 & 3,5 & 3,5 & 3,7 & 3,7 & 3,7 & 3,8 & 4,2 & 3,9 & 4,3 & 4,4 & 4,6 & 5,0 & \\
\hline Courant & 99,7 & 99,7 & 99,7 & 99,6 & 99,6 & 99,6 & 99,5 & 94,7 & 93,2 & 92,1 & 92,3 & 92,2 & 92,3 & 83,9 & 82,6 & 80,4 & 81,6 & 80,4 & 80,3 & 79,7 & 76,9 & 72,9 \\
\hline Non-courant & 0,3 & 0,3 & 0,3 & 0,4 & 0,4 & 0,4 & 0,5 & 5,3 & 6,8 & 7,9 & 7,7 & 7,8 & 7,7 & 16,1 & 17,4 & 19,6 & 18,4 & 19,6 & 19,7 & 20,3 & 23,1 & $\begin{array}{l}27,1 \\
35,8\end{array}$ \\
\hline Courant/variable & 83,4 & 83,5 & 83,6 & 81,6 & 84,7 & 81,9 & 78,8 & 76,9 & 74,8 & 69,9 & 65,8 & 63,7 & 65,4 & 63,4 & 59,8 & 56,2 & 58,2 & 55,9 & 55,6 & 54,3 & 47,9 & $\begin{array}{l}35,8 \\
330\end{array}$ \\
\hline $\begin{array}{l}\text { Courantitie } \\
\text { Non-courant/variable }\end{array}$ & 13,5 & 13,8 & 13,5 & 15,3 & 12,4 & & 17,8 & 15,0 & 15,5 & & 23,0 & 24,8 & & & & 20,0 & & & 20,3 & 20,8 & & \\
\hline Non-courant/fixe & 0,3 & 0,3 & 0,3 & 0,4 & 0,4 & 0,4 & 0,5 & 5,3 & 6,8 & 7,9 & 7,7 & 7,8 & 7,7 & 16,1 & 17,4 & 19,6 & 18,4 & 19,6 & 19,7 & 20,3 & 23,1 & 27,1 \\
\hline Autres produits & 2,8 & 2,4 & & 2,7 & $\begin{array}{r}2,5 \\
075 \\
075\end{array}$ & $\begin{array}{r}2,5 \\
975 \\
97\end{array}$ & 3,0 & 2,8 & $\begin{array}{r}2,9 \\
071\end{array}$ & 3,5 & $\begin{array}{r}3,5 \\
095\end{array}$ & 3,7 & 3,7 & 3,7 & $\begin{array}{r}3,8 \\
700\end{array}$ & 4,2 & $\begin{array}{r}3,9 \\
789\end{array}$ & 4,3 & 4,4 & 4,6 & 5,0 & $\begin{array}{r}6,2 \\
685 \\
685\end{array}$ \\
\hline 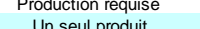 & $\begin{array}{l}97,2 \\
85,6\end{array}$ & $\begin{array}{l}97,6 \\
85,6\end{array}$ & $\begin{array}{l}97,5 \\
856\end{array}$ & $\begin{array}{l}97,3 \\
83,8\end{array}$ & $\begin{array}{l}97,5 \\
865\end{array}$ & $\begin{array}{r}97,5 \\
887\end{array}$ & $\begin{array}{l}97,0 \\
81,5\end{array}$ & 97,2 & 97,1 & $\begin{array}{l}96,5 \\
718\end{array}$ & 96,5 & 96,3 & $\begin{array}{l}96,3 \\
678\end{array}$ & 81,1 & 79,9 & 77,4 & 78,9 & 77,4 & 77,1 & $\begin{array}{l}76,5 \\
581\end{array}$ & 73,4 & $\begin{array}{l}68,5 \\
40,3\end{array}$ \\
\hline Un groupe de produits & $\begin{array}{l}85,0 \\
10,0\end{array}$ & $\begin{array}{l}85,6 \\
10,4\end{array}$ & $\begin{array}{l}85,6 \\
10,3\end{array}$ & $\begin{array}{l}83,8 \\
11,6\end{array}$ & $\begin{array}{r}\begin{array}{r}60,5 \\
9,0\end{array} \\
9\end{array}$ & $\begin{array}{l}84, / \\
10,6\end{array}$ & $\begin{array}{l}81,5 \\
12,9\end{array}$ & $\begin{array}{l}19,3 \\
10,7\end{array}$ & $\begin{array}{l}6,99 \\
11,8\end{array}$ & $\begin{array}{l}1,8 \\
15,1\end{array}$ & $\begin{array}{l}68,0 \\
19.3\end{array}$ & $\begin{array}{l}60,9 \\
20.9\end{array}$ & $\begin{array}{l}6 /, 8 \\
19,6\end{array}$ & $\begin{array}{l}60,2 \\
12,9\end{array}$ & $\begin{array}{l}63,1 \\
14,1\end{array}$ & $\begin{array}{l}60,8 \\
14,2\end{array}$ & $\begin{array}{l}62,3 \\
14,4\end{array}$ & $\begin{array}{l}5,9 \\
15,1\end{array}$ & $\begin{array}{l}59,4 \\
15,3\end{array}$ & $\begin{array}{l}58,1 \\
158\end{array}$ & $\begin{array}{l}52,4 \\
180\end{array}$ & $\begin{array}{l}40,3 \\
24,5\end{array}$ \\
\hline Tous les produits & 1,7 & 1,6 & 1,6 & 1,9 & 2,0 & 2,2 & 2,6 & 7,1 & 8,4 & 9,7 & 9,1 & 9,1 & 8,9 & 2,0 & 2,2 & 2,3 & 2,2 & 2,4 & 2,5 & 2,6 & 3,0 & $\begin{array}{r}r 4,5 \\
3,7\end{array}$ \\
\hline $\begin{array}{l}\text { Production facultati } \\
\end{array}$ & 2,8 & 2,4 & 2,5 & 2,7 & 2,5 & 2,5 & 3,0 & 2,8 & 2,9 & 3,5 & 3,5 & 3,7 & 3,7 & 18,9 & 20,1 & 22,6 & 21,1 & 22,6 & 22.9 & 23.5 & 26.6 & 31,5 \\
\hline \multicolumn{23}{|l|}{$\begin{array}{l}\text { Propouction deculatulu } \\
\text { Proporte che }\end{array}$} \\
\hline Avec limites & 33,4 & 32,9 & 32,6 & 29,5 & 32,6 & 31,3 & 33,3 & 28,6 & 27,8 & 24,7 & 30,5 & 29,6 & 30,2 & 31,5 & 20,6 & 20,9 & 22,6 & 18,9 & 16,2 & 17,4 & 13,3 & \\
\hline Sans limites & 63,8 & & & 67,8 & 65,0 & & 63,8 & 69,0 & & 72,6 & 66,8 & 67,6 & 67,0 & & 76,7 & 76,3 & 74,8 & 78,4 & 81,2 & 79,9 & & \\
\hline Fixe & 13,8 & 14,2 & 13,9 & 15,7 & 12,8 & 15 & 18,3 & 20,2 & 22,4 & 26,7 & 30,7 & 32,6 & 30,9 & 32,9 & 36,4 & 39,6 & 37,8 & 39,8 & 40,0 & 41,1 & 47,1 & 58,0 \\
\hline & 83,4 & 83,5 & 83,6 & 81,6 & 84,7 & 81,9 & 78,8 & 76,9 & 74,8 & & 65,8 & 63,7 & & & 59,8 & & & & & & 47,9 & 35,8 \\
\hline Avec c. & $\begin{array}{l}3,4 \\
13\end{array}$ & $\begin{array}{l}3,7 \\
12\end{array}$ & $\begin{array}{l}3,9 \\
11\end{array}$ & $\begin{array}{l}4,7 \\
13\end{array}$ & $\begin{array}{l}4,5 \\
13\end{array}$ & $\begin{array}{l}4,7 \\
12\end{array}$ & $\begin{array}{l}5,7 \\
24\end{array}$ & $\begin{array}{r}12,5 \\
31\end{array}$ & 14,7 & $\begin{aligned} 17,1 \\
55\end{aligned}$ & 17,7 & 17,6 & 16,6 & 24,0 & 26,1 & $\begin{array}{l}28,7 \\
5,\end{array}$ & $\begin{array}{l}27,6 \\
54\end{array}$ & 29,4 & 29,7 & 30,5 & 34,7 & $\begin{array}{l}43,5 \\
94\end{array}$ \\
\hline & $\begin{array}{l}1,3 \\
9,9\end{array}$ & $\begin{array}{r}1,2 \\
10,0\end{array}$ & $\begin{array}{l}1,1 \\
9,7\end{array}$ & $\begin{array}{l}1,3 \\
10,8\end{array}$ & $\begin{array}{l}1,3 \\
8,1\end{array}$ & $\begin{array}{r}1,2 \\
10,9\end{array}$ & $\begin{array}{r}2,4 \\
11,6\end{array}$ & & & $\begin{array}{l}5,5 \\
6,0\end{array}$ & & $\begin{array}{r}11,4 \\
5.4\end{array}$ & $\begin{array}{r}11,0 \\
5,1\end{array}$ & $\begin{array}{l}4,4 \\
6,3 \\
6\end{array}$ & & & $\begin{array}{l}5,4 \\
73\end{array}$ & & $\begin{array}{l}6,3 \\
7,1\end{array}$ & $\begin{array}{l}6,1 \\
72\end{array}$ & 84 & $\begin{array}{l}9,4 \\
9,9\end{array}$ \\
\hline $\begin{array}{l}\text { Sans contrainie } \\
\text { Un seul produit }\end{array}$ & $\begin{array}{l}9,9 \\
85,6\end{array}$ & $\begin{array}{l}10,0 \\
85,6\end{array}$ & $\begin{array}{l}9,1 \\
85,6\end{array}$ & $\begin{array}{l}10,8 \\
83.8\end{array}$ & $\begin{array}{r}86,1 \\
86,5\end{array}$ & $\begin{array}{l}10,9 \\
84,7\end{array}$ & $\begin{array}{l}11,6 \\
81,5\end{array}$ & $\begin{array}{l}6,1 \\
79,3\end{array}$ & $\begin{array}{r}5,6 \\
76,9\end{array}$ & $\begin{array}{l}0,0 \\
71,8\end{array}$ & $\begin{array}{l}58,2 \\
68,0\end{array}$ & $\begin{array}{l}56,4 \\
66,3 \\
\end{array}$ & $\begin{array}{l}5,1 \\
67.8\end{array}$ & $\begin{array}{r}6,3 \\
66,2\end{array}$ & 63.7 & 60,8 & 62,3 & $\begin{array}{l}1,1 \\
59,9\end{array}$ & $\begin{array}{l}7,1 \\
59,4\end{array}$ & $\begin{array}{l}1,2 \\
58,1\end{array}$ & $\begin{array}{r}82,4 \\
52,4\end{array}$ & $\begin{array}{r}9,9 \\
40,3\end{array}$ \\
\hline roupe de produits & $\begin{array}{l}\begin{array}{l}05,0 \\
10,0\end{array} \\
\end{array}$ & $\begin{array}{l}\begin{array}{l}0.0,0 \\
10,4\end{array} \\
\end{array}$ & $\begin{array}{l}\begin{array}{l}\quad 0,0 \\
10,3\end{array} \\
\end{array}$ & $\begin{array}{l}\begin{array}{l}0.0,0 \\
11,6\end{array} \\
\end{array}$ & $\begin{aligned} 0,0,0 \\
9,0\end{aligned}$ & 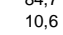 & $\begin{array}{l}01,9 \\
12,9\end{array}$ & $\begin{array}{l}10,7 \\
10,7\end{array}$ & $\begin{array}{l}10,9 \\
11,8\end{array}$ & $\begin{array}{l}1,0 \\
15,1\end{array}$ & $\begin{array}{l}19,3 \\
19,0\end{array}$ & $\begin{array}{l}20,9 \\
20,9\end{array}$ & 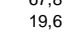 & $\begin{array}{l}l_{0,2} \\
12,9\end{array}$ & 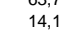 & $\begin{array}{l}\quad 14,2 \\
14,2\end{array}$ & $\begin{array}{l}14,4 \\
14,4\end{array}$ & 15,1 & $\begin{array}{l}15,3 \\
15,3\end{array}$ & $\begin{array}{l}15,8 \\
15,1\end{array}$ & $\begin{array}{l}5,4,4 \\
18,0\end{array}$ & $\begin{array}{l}24,5 \\
24,5 \\
4\end{array}$ \\
\hline roduits & 1,7 & 1,6 & 1,6 & 1,9 & 2,0 & 2,2 & 2,6 & 7,1 & 8,4 & 9,7 & 9,1 & 9,1 & 8,9 & 2,0 & 2,2 & 2,3 & 2,2 & 2,4 & 2,5 & 2,6 & 3,0 & 3,7 \\
\hline erficie & 3,0 & 2,9 & 3,3 & 3,8 & 3,4 & 3,2 & 3,6 & 4,8 & 5,1 & 7,4 & 12,1 & 13,4 & 12,0 & 19,0 & 20,4 & 21,4 & 20,0 & 21,3 & 21,6 & 22,1 & 25,2 & 29,7 \\
\hline $\begin{array}{l}\text { Nbre d'animaux } \\
\text { Recettes }\end{array}$ & 4,2 & 4,4 & 4,4 & 5,4 & 5,2 & 6,4 & 6,6 & 5,5 & 5,4 & 6,1 & 6,3 & 6,4 & 6,4 & 8,8 & 9,6 & 10,7 & 10,9 & 11,8 & 11,9 & 12,4 & 14,2 & 19,6 \\
\hline & & - & 0,5 & 1,7 & 1,1 & 2,9 & 4,5 & 4,9 & 6,3 & 7,2 & 6,9 & 6,9 & 6,8 & - & - & - & - & - & - & - & - & - \\
\hline $\begin{array}{l}\text { Avec exceptions } \\
\text { Sans exceptions }\end{array}$ & & & & & & & & & & & & & & 15,2 & 16,3 & 18,4 & 17,2 & 18.3 & 18,5 & 18,9 & 21,6 & 25,3 \\
\hline
\end{tabular}


Tableau annexe 20. Turquie : composition de l'ESP par catégorie et étiquette, 1986-2007

Part en pourcentage de l'ESP

\begin{tabular}{|c|c|c|c|c|c|c|c|c|c|c|c|c|c|c|c|c|c|c|c|c|c|c|}
\hline & 1986 & 1987 & 1988 & 1989 & 1990 & 1991 & 1992 & 1993 & 1994 & 1995 & 1996 & 1997 & 1998 & 1999 & 2000 & 2001 & 2002 & 2003 & 2004 & 2005 & 2006 & 2007 \\
\hline PSE (millions TRY) & 2 & 3 & 4 & 9 & 17 & 36 & 54 & 85 & 111 & 192 & 454 & 1264 & 2686 & 3133 & 4099 & 947 & 9011 & 16153 & 16251 & 16920 & 15458 & 17468 \\
\hline \multicolumn{23}{|c|}{ Composition par catégorie avec labels correspondants } \\
\hline Production & 74,0 & 76,3 & 62,3 & 70,0 & 81,8 & 84,7 & 80,8 & 82,0 & 49,2 & 50,7 & 61,5 & 75,6 & & & 87,1 & 57,0 & & 82,7 & 81,9 & 82,6 & 73,9 & \\
\hline Intrants & 26,0 & 23,7 & 37,7 & 30,0 & 18,2 & 15,3 & 19,2 & 18,0 & 47,2 & 47,8 & 37,2 & 24,2 & 17,8 & 22,8 & 12,5 & 31,4 & 3,6 & 2,1 & 2,8 & 3,2 & 8,2 & 7,6 \\
\hline $\mathrm{S} / \mathrm{Na} / \mathrm{Rc} / \mathrm{Rv}$ & & & & & & & & & 3,6 & 1,5 & 1,4 & 0,2 & 0,1 & 0,1 & 0,4 & 11,6 & 21,3 & 15,3 & 15,3 & 14,2 & 17,9 & 15,1 \\
\hline 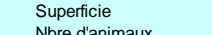 & - & - & $\cdot$ & - & - & - & - & - & 3,6 & 1,5 & 1,4 & 0,2 & 0,1 & 0,1 & 0,4 & 11,6 & 21,3 & 15,3 & 15,3 & 14,2 & 17,8 & 14,9 \\
\hline $\begin{array}{l}\text { Nore d'anima } \\
\text { Recettes }\end{array}$ & $:$ & : & : & : & - & . & - & - & - & - & - & - & - & - & - & 0,0 & 0,0 & 0,0 & 0,0 & 0,0 & 0,0 & 0,2 \\
\hline \multirow{2}{*}{\multicolumn{23}{|c|}{$\begin{array}{l}\text { Revenu } \\
\text { Autres produits }\end{array}$}} \\
\hline & 100,0 & 100,0 & 100,0 & 100,0 & 100,0 & 100,0 & 100,0 & 100,0 & 100,0 & 100,0 & 100,0 & 100,0 & 100,0 & 100,0 & 100,0 & 91,2 & 79,2 & 85,0 & 84,7 & 86,1 & 82,6 & 85,4 \\
\hline Non-courant & & & & & & & & & & & & & & & & 8,8 & 20,8 & 15,0 & 15,3 & 13,9 & 17,4 & 14,6 \\
\hline Courant/variable & 82,9 & 83,9 & 79,2 & 83,0 & 91,1 & 93,2 & 93,7 & 87,6 & 88,0 & 89,1 & 85,7 & 88,5 & 92,3 & 89,9 & 89,1 & 0,8 & 73,3 & 81,0 & 78,9 & & 64,2 & 66,3 \\
\hline $\begin{array}{l}\text { Courant/fixe } \\
\text { Non-courant/variable }\end{array}$ & 17,1 & 16,1 & 20,8 & 17,0 & 8,9 & 6,8 & 6,3 & 12,4 & 12,0 & 10,9 & 14,3 & 11,5 & 7,7 & 10,1 & 10,9 & & 5,9 & 4,0 & 5,8 & 9,3 & 18,4 & \\
\hline $\begin{array}{l}\text { Non-courant fixe } \\
\text { Antres nondlits }\end{array}$ & - & - & - & - & - & - & - & - & - & - & - & - & - & - & - & 8,8 & 20,8 & 15,0 & 15,3 & 13,9 & 17,4 & 14,6 \\
\hline $\begin{array}{l}\text { Autres proaduls } \\
\text { Production requise }\end{array}$ & 100,0 & 100,0 & 100,0 & 100,0 & 100,0 & 100,0 & 100,0 & 100,0 & 100,0 & 100,0 & 100,0 & 100,0 & 100,0 & 100,0 & 100,0 & 91,2 & 79,2 & 85,0 & 84.7 & 86.1 & 82,6 & \\
\hline Un seul produit & 74,4 & 76,6 & 62,6 & 70,5 & 82,2 & 85,1 & 81,0 & 82,3 & 53,1 & 52,6 & 63,1 & 76,0 & 82,3 & 77,3 & 87,9 & 64,9 & 76,5 & 83,5 & 82,7 & 84,1 & 77,8 & $\begin{array}{l}80,8 \\
80,8\end{array}$ \\
\hline Un groupe de produits & 16,8 & 15,3 & 19,9 & 16,0 & 7,8 & 6,0 & 5,8 & 6,5 & 10,8 & 9,2 & 12,6 & 9,6 & 7,1 & 6,4 & 5,0 & 26,2 & 2,7 & 1,5 & 2,0 & 1,7 & 3,8 & 3,3 \\
\hline Tous les produits & 8,9 & 8,1 & 17,4 & 13,5 & 10,0 & 8,9 & 13,2 & 11,2 & 36,1 & 38,3 & 24,3 & 14,3 & 10,6 & 16,3 & 7,1 & 0,0 & 0,0 & 0,0 & 0,1 & 0,2 & 1,0 & 1,3 \\
\hline \multirow{2}{*}{\multicolumn{23}{|c|}{$\begin{array}{l}\text { Production facultative } \\
\text { Proportion de chaque label }\end{array}$}} \\
\hline & 74.5 & & 62.9 & & 82.2 & 85.2 & 81,1 & 82.3 & & & 629 & 758 & & & 875 & & & & & & & \\
\hline $\begin{array}{l}\text { Avec umimes } \\
\text { Sans limites }\end{array}$ & 26.0 & $\begin{array}{l}10,1 \\
23,7\end{array}$ & 37,7 & $\begin{array}{l}7,1 \\
30,0\end{array}$ & 18.2 & $\begin{array}{l}15,3 \\
15,\end{array}$ & $\begin{array}{l}19,2 \\
19.2\end{array}$ & $\begin{array}{l}18,0 \\
0\end{array}$ & $\begin{array}{l}4,2 \\
47,2\end{array}$ & $\begin{array}{l}5,4 \\
47,8\end{array}$ & $\begin{array}{l}37,2 \\
37.2\end{array}$ & 24.2 & $\begin{array}{l}82,2 \\
17,8\end{array}$ & 22,8 & $\begin{array}{l}12.5 \\
12.5\end{array}$ & $\begin{array}{l}0,1 \\
31,4\end{array}$ & $\begin{array}{r}90,4 \\
3,6\end{array}$ & 2,1 & $\begin{aligned} 9,2 \\
2.8\end{aligned}$ & $\begin{array}{c}90,6 \\
3.2\end{array}$ & $\begin{array}{r}\begin{array}{r}1,6 \\
8.2\end{array} \\
\end{array}$ & $\begin{array}{r}92,4 \\
7,6\end{array}$ \\
\hline Fixe & 17,1 & 16,1 & 20,8 & 17,0 & 8,9 & 6,8 & 6,3 & 12,4 & 12,0 & 10,9 & 14,3 & 11,5 & 7,7 & 10,1 & 10,9 & 99,2 & 26,7 & 19,0 & 21,1 & 23,2 & 35,8 & $\begin{array}{r}33,6 \\
33,7\end{array}$ \\
\hline Variable & 82,9 & 83,9 & 79,2 & 83,0 & 91,1 & 93,2 & 93,7 & 87,6 & 88,0 & 89,1 & 85,7 & 88,5 & 92,3 & 89,9 & 89,1 & 0,8 & 73,3 & 81,0 & 78,9 & 76,8 & 64,2 & \\
\hline \multicolumn{23}{|l|}{$\begin{array}{l}\text { avabale } \\
\text { Avec c. (obligatoire) } \\
\text { Avec c. (volontaire) }\end{array}$} \\
\hline Sans contrainte & 26,0 & 24,2 & 38,2 & 30,4 & 18,9 & 15,7 & 19,5 & 23,6 & 51,7 & 50,8 & 40,0 & 26,0 & 18,4 & 26,5 & 18,4 & 101,9 & 27,2 & 19,2 & 21,1 & 23,7 & $\begin{array}{r}3,0 \\
37,2\end{array}$ & $\begin{array}{r}35,4 \\
35,4\end{array}$ \\
\hline Un seul produit & 74,4 & 76,6 & 62,6 & 70,5 & 82,2 & 85,1 & 81,0 & 82,3 & 53,1 & 52,6 & 63,1 & 76,0 & 82,3 & 77,3 & 87,9 & 64,9 & 76,5 & 83,5 & 82,7 & 84,1 & 77,8 & 80,8 \\
\hline Un groupe de produits & 16,8 & 15,3 & 19,9 & 16,0 & 7,8 & 6,0 & 5,8 & 6,5 & 10,8 & 9,2 & 12,6 & 9,6 & 7,1 & & 5,0 & 26,2 & & 1,5 & 2,0 & 1,7 & 3,8 & 3,3 \\
\hline Tous les produits & 8,9 & 8,1 & 17,4 & 13,5 & 10,0 & 8,9 & 13,2 & 11,2 & 36,1 & 38,3 & 24,3 & 14,3 & 10,6 & 16,3 & 7,1 & 0,0 & 0,0 & 0,0 & 0,1 & 0,2 & 1,0 & 1,3 \\
\hline $\begin{array}{l}\text { Superficie } \\
\text { Nbre d'animaux }\end{array}$ & - & $\therefore$ & $\therefore$ & $\therefore$ & $\therefore$ & . & & & 3,6 & 1,5 & 1,4 & 0,2 & 0,1 & 0,1 & 0,4 & 11,6 & 21,3 & 15,3 & 15,3 & 14,2 & 17,8 & 14,9 \\
\hline Recettes & . & . & - & - & . & . & - & - & - & - & - & - & - & - & - & 0,0 & 0,0 & 0,0 & 0,0 & 0,0 & 0,0 & 0,2 \\
\hline & - & - & - & - & - & - & - & - & - & - & - & - & - & - & - & & & & & & & \\
\hline $\begin{array}{l}\text { Avec exceptions } \\
\text { Sans exceptions }\end{array}$ & - & - & - & - & - & : & & & : & : & - & : & $\because$ & : & & 8,8 & 20,8 & 15,0 & 15,3 & 13.9 & 17,4 & 14.6 \\
\hline
\end{tabular}


Tableau annexe 21. États-Unis : composition de l'ESP par catégorie et étiquette, 1986-2007

Part en pourcentage de l'ESP

\begin{tabular}{|c|c|c|c|c|c|c|c|c|c|c|c|c|c|c|c|c|c|c|c|c|c|c|}
\hline & 1986 & 1987 & 1988 & 1989 & 1990 & 1991 & 1992 & 1993 & 1994 & 1995 & 1996 & 1997 & 1998 & 1999 & 2000 & 2001 & 2002 & 2003 & 2004 & 2005 & 2006 & $\frac{2007}{20603}$ \\
\hline$\frac{P S E \text { (millions USD) }}{2}$ & 38377 & 39902 & 32067 & 39437 & 31943 & 31310 & 31762 & 33909 & 29651 & 20805 & 29533 & 30444 & 46993 & 55997 & 52829 & 51523 & 40746 & 36036 & 42956 & 41024 & 30860 & 32663 \\
\hline \multicolumn{23}{|c|}{ Composition par catégorie avec labels correspondants } \\
\hline Production & 45,0 & 47,8 & 41,7 & 56,7 & 47,5 & 49,7 & 48,8 & 50,4 & 49,7 & 44,9 & 48,9 & 47,0 & 54,4 & 56,5 & 52,5 & 53,4 & 41,5 & 36,6 & & 35,5 & & \\
\hline Intrants & 18,3 & 17,6 & 22,3 & 19,8 & 23,0 & 22,8 & 21,3 & 20,1 & 22,8 & 31,8 & 23,2 & 22,6 & 14,5 & 13,1 & 14,6 & 16,2 & 19,5 & 23,9 & 20,9 & 23,0 & 30,6 & \\
\hline $\mathrm{S} / \mathrm{Na} / \mathrm{Rc} / \mathrm{Rv}$ & 36,6 & 32,9 & 32,8 & 20,0 & 24,7 & 22,2 & 24,7 & 24,8 & 21,2 & 14,5 & 21,9 & 24,5 & 26,7 & 27,4 & 29,7 & 26,9 & 33,8 & 33,6 & 31,8 & 36,0 & 35,4 & 27,2 \\
\hline Superficie & 33,0 & 31,0 & 28,5 & 17,9 & 22,6 & 19,6 & 22,5 & 23,4 & 19,5 & 11,9 & 19,9 & 21,1 & 23,8 & 24,5 & 26,2 & 22,2 & 29,1 & 23,4 & 27,1 & 29,6 & 27,4 & 20,5 \\
\hline Nbre d'animaux & 0,0 & 0,0 & 2,5 & 0,0 & 0,0 & 0,0 & 0,0 & 0,0 & 0,0 & 0,0 & 0,0 & 0,0 & 0,3 & 0,0 & 0,0 & 0,0 & 0,1 & 0,0 & 0,0 & 0,0 & 0,0 & \\
\hline Recettes & & & & & & & & & & & & & & & 0,0 & 0,0 & 0,3 & 3,4 & 0,1 & 2,6 & 3,2 & 2,9 \\
\hline $\begin{array}{l}\text { Revenu } \\
\text { Autres roduite }\end{array}$ & 3,6 & 1,9 & 1,8 & 2,1 & 2,1 & 2,6 & 2,2 & 1,5 & 1,7 & 2,7 & 2,0 & 3,4 & 2,7 & 2,8 & 3,6 & 4,6 & $\begin{array}{l}4,4 \\
\end{array}$ & 6,8 & 4,6 & 3,8 & 4,9 & $\begin{array}{ll}3,7 \\
69\end{array}$ \\
\hline Courant & 0,1 & $\frac{1,1}{993}$ & $\frac{1,5}{995}$ & 0,0 & 10,0 & $\frac{3,0}{100}$ & $\frac{3,0}{100}$ & 10,1 & 0,0 & 0,1 & $\frac{0,1}{884}$ & $\frac{1,9}{794}$ & 年, & 8,95 & $\frac{1,2}{80,4}$ & 然, & 年, & sol & $\frac{4,9}{777}$ & $\frac{1,5}{724}$ & $\frac{1,8}{75,1}$ & $\begin{array}{r}6,9 \\
78,4\end{array}$ \\
\hline Non-courant & 1,5 & 0,7 & $\begin{array}{r}9,5 \\
0,5\end{array}$ & 100,0 & 100,0 & 100,0 & 100,0 & 100,0 & 100,0 & 100,0 & $\begin{array}{l}82,4 \\
17,6\end{array}$ & $\begin{array}{l}20,4 \\
20,6\end{array}$ & $\begin{array}{l}8,0 \\
18,0\end{array}$ & $\begin{array}{l}80,5 \\
19,5\end{array}$ & $\begin{array}{l}80,1 \\
19,9\end{array}$ & $\begin{array}{l}83,0 \\
17,0\end{array}$ & $\begin{array}{l}82,6 \\
17,4\end{array}$ & $\begin{array}{l}80,5 \\
19,5\end{array}$ & $\begin{array}{l}2,1,1 \\
22,3\end{array}$ & $\begin{array}{l}73,4 \\
26,6\end{array}$ & $\begin{array}{l}24,1 \\
24.9\end{array}$ & $\begin{array}{l}78,4 \\
21,6\end{array}$ \\
\hline Courant variable & 79,8 & 79,1 & 66,0 & 76,1 & 74,4 & 72,5 & 72,8 & 69,9 & 71,1 & 62,2 & 54,9 & 50,8 & 58,2 & 61,1 & 56,9 & 58,8 & 51,5 & $\begin{array}{ll}44.1 \\
\end{array}$ & $\begin{array}{lll}47.4 & \end{array}$ & 40,3 & 34.9 & $\begin{array}{l}21,6 \\
42,6\end{array}$ \\
\hline Courantfixe & 18,6 & 18,5 & 30,2 & 20,4 & 20,8 & 22,2 & 21,9 & 25,4 & 22,6 & 29,1 & 21,5 & 22,7 & 19,5 & 16,5 & 19,9 & 20,7 & 26,0 & 30,5 & 25,3 & 27,6 & 32,4 & $\begin{array}{l}42,6 \\
28,9\end{array}$ \\
\hline Non-courant/variable & & & & & & & & & & & & & 6,0 & 9,8 & 10,3 & 9,0 & 4,4 & 1,5 & 10,0 & 11,6 & 5,0 & $\begin{aligned} 2,3,9 \\
2,9\end{aligned}$ \\
\hline Non-courant fixe & 1,5 & 0,7 & 0,5 & & & & & & & & 17,6 & 20,6 & 12.0 & 9.8 & 9,6 & 8.0 & 13,0 & 18,0 & 12,3 & 15.0 & 19,9 & 18,8 \\
\hline Autres produits & 0,1 & 1,7 & 3,3 & 3,5 & 4,8 & 5,3 & 5,3 & 4,7 & 6,3 & 8,7 & 6,1 & 5,9 & 4,3 & 2,9 & 3,2 & 3,6 & 5,1 & 5,9 & 4,9 & 5,5 & 7,8 & $\begin{array}{l}\quad 0,6 \\
6,9 \\
\end{array}$ \\
\hline Production requise & 98,4 & 97,6 & 96,2 & 96,5 & 95,2 & 94,7 & 94,7 & 95,3 & 93,7 & 91,3 & 76,4 & 73,4 & 77,7 & 77,5 & 76,8 & 79,5 & 77,5 & 74,6 & 72,7 & 67,9 & 67,3 & 71,5 \\
\hline Un seul produit & 74,9 & 78,1 & 61,6 & 70,8 & 68,7 & 67,3 & 69,0 & 66,4 & 67,1 & 56,6 & 51,0 & 47,4 & 56,3 & 59,3 & 55,1 & 56,8 & 49,0 & 41,3 & 45,0 & 37,6 & 31,4 & 39,4 \\
\hline $\begin{array}{l}\text { Un groupe de } \\
\text { Tous les produ }\end{array}$ & 4,2 & 2,9 & 14,3 & 8,2 & 5,7 & 6,3 & 6,6 & 11,4 & 6,5 & 5,6 & 4,1 & 3,9 & 6,3 & 4,7 & 5,9 & 4,8 & 7,4 & 6,9 & 5,6 & 7,5 & 5,9 & 4,1 \\
\hline 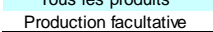 & 列 & $\begin{array}{r}16,6 \\
2,4\end{array}$ & $\begin{array}{l}20,2 \\
3.8\end{array}$ & $\begin{array}{r}7,5 \\
3,5\end{array}$ & $\begin{array}{l}20,8 \\
4.8\end{array}$ & $\begin{array}{c}21,1 \\
5,3\end{array}$ & $\begin{array}{r}19,1 \\
5,3\end{array}$ & $\begin{array}{r}17,5 \\
4,7\end{array}$ & $\begin{array}{c}20,2 \\
6,3\end{array}$ & $\begin{array}{r}29,0 \\
8,7\end{array}$ & $\begin{array}{l}21,2 \\
23,6\end{array}$ & $\begin{array}{l}22,2 \\
26,6\end{array}$ & 22.3 & $\begin{array}{l}13,6 \\
22,5\end{array}$ & $\begin{array}{l}15,8 \\
23,2\end{array}$ & $\begin{array}{l}17,8 \\
20,5\end{array}$ & 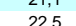 & $\begin{array}{l}26,5 \\
254\end{array}$ & & $\begin{array}{l}22,8 \\
32,1\end{array}$ & $\begin{array}{l}30,0 \\
327\end{array}$ & $\begin{array}{l}28,0 \\
28,5\end{array}$ \\
\hline \multicolumn{23}{|c|}{$\begin{array}{l}\text { Production facultative } \\
\text { Proportion de chaque label }\end{array}$} \\
\hline Avec limites & 80,5 & 83,4 & 63,5 & 74,4 & 73,7 & 71,7 & 73,2 & 68,3 & 73,4 & 59,7 & 72,0 & 72,8 & 75,0 & 75,9 & 69,6 & 69,0 & 61,4 & 60,7 & 67,4 & 66,3 & 56,1 & 67,8 \\
\hline ns limites & 19,5 & & 36,5 & 25,6 & 26,3 & 28,3 & 26,8 & 31,7 & 26,6 & & 28,0 & 27,2 & 25,0 & 24,1 & 30,4 & 31,0 & 38,6 & 39,3 & 32,6 & & 43,9 & 32,2 \\
\hline $\begin{array}{l}\text { Fixe } \\
\text { Variable }\end{array}$ & $\begin{array}{l}20,1 \\
798\end{array}$ & 19,2 & 30,7 & 20,4 & $\begin{array}{l}20,8 \\
744\end{array}$ & $\begin{array}{ll}22,2 \\
725\end{array}$ & $\begin{array}{l}21,9 \\
72,8\end{array}$ & $\begin{array}{l}25,4 \\
69,9\end{array}$ & $\begin{array}{l}22,6 \\
711\end{array}$ & $\begin{array}{l}29,1 \\
62,2\end{array}$ & $\begin{array}{l}39,1 \\
54,9\end{array}$ & $\begin{array}{l}43,3 \\
50,8\end{array}$ & $\begin{array}{l}31,5 \\
642\end{array}$ & $\begin{array}{l}26,3 \\
70,8\end{array}$ & $\begin{array}{l}29,5 \\
672\end{array}$ & $\begin{array}{l}28,7 \\
67,8\end{array}$ & $\begin{array}{l}39,0 \\
550\end{array}$ & 48,5 & 37,7 & $\begin{array}{l}42,7 \\
518\end{array}$ & 52,3 & $\begin{array}{l}47,6 \\
455\end{array}$ \\
\hline & $\begin{array}{l}9,8 \\
217\end{array}$ & $\begin{array}{l}9,1 \\
15,9\end{array}$ & $\begin{array}{l}6,0 \\
25,5\end{array}$ & $\begin{array}{l}76,1 \\
12,6\end{array}$ & $\begin{array}{l}7,4 \\
107\end{array}-7$ & $\begin{array}{r}2,5 \\
9,4\end{array}$ & $\begin{array}{r}2,8 \\
8,9\end{array}$ & $\begin{array}{l}69,9 \\
15,7\end{array}$ & $\begin{array}{r}17,1 \\
7,2\end{array}$ & $\begin{array}{l}62,2 \\
10,9\end{array}$ & $\begin{array}{l}54,9 \\
24,4\end{array}$ & 50,8 & $\begin{array}{l}64,2,2 \\
354-2\end{array}$ & $\begin{array}{l}0,8 \\
455\end{array}$ & $\begin{array}{l}67,2 \\
47,8\end{array}$ & $\begin{array}{l}67,8 \\
425\end{array}$ & 55,9 & $\begin{array}{l}45,6 \\
354\end{array}$ & $\begin{array}{l}5 /, 4 \\
443,3\end{array}$ & $\begin{array}{l}51,8 \\
485\end{array}$ & $\begin{array}{r}39,9 \\
385\end{array}$ & $\begin{array}{l}45,5 \\
270\end{array}$ \\
\hline c. (volontaire) & 0,5 & 2,2 & $\begin{array}{r}3,9 \\
3,9\end{array}$ & 4,0 & 5,5 & $\begin{array}{l}3,4 \\
5,9\end{array}$ & 6,0 & $\begin{array}{r}5,1 \\
5,4\end{array}$ & 7,0 & 9,3 & $\begin{array}{c}24,4 \\
6,9\end{array}$ & $\begin{array}{c}26,1 \\
6,8\end{array}$ & $\begin{array}{l}0,4,7 \\
4,7 \\
0.7\end{array}$ & 3,3 & $\begin{array}{r}\begin{array}{r}1,0 \\
3,6\end{array} \\
\end{array}$ & 4,0 & $\begin{array}{l}5,4 \\
6,1\end{array}$ & 6,8 & $\begin{array}{r}44,3 \\
7,1\end{array}$ & $\begin{array}{r}40,5 \\
8,5\end{array}$ & $\begin{array}{l}0,0,0 \\
11,3\end{array}$ & $\begin{array}{l}27,0 \\
10,5\end{array}$ \\
\hline Sans contrainte & 45,4 & 40,6 & 33,0 & 28,6 & 38,5 & 35,5 & 37,7 & 29,5 & 37,1 & 35,2 & 20,1 & 21,1 & 14,5 & 13,5 & 15,6 & 18,2 & 20,4 & 29,0 & 19,8 & 22,4 & 29,9 & 26,1 \\
\hline Seur produm & 74,9 & 78,1 & 61,6 & 70,8 & 68,7 & 67,3 & 69,0 & 66,4 & 67,1 & 56,6 & 51,0 & 47,4 & 56,3 & 59,3 & 55,1 & 56,8 & 49,0 & 41,3 & 45,0 & 37,6 & 31,4 & 39,4 \\
\hline ede produlits & 4,2 & 2,9 & 14,3 & 8,2, & 5,7 & 6,3 & 6,6 & 11,4 & 6,5 & 5,6 & 4,1 & 3,9 & 6,3 & 4,7 & 5,9 & 4,8 & 7,4 & 6,9 & 5,6 & 7,5 & 5,9 & 4,1 \\
\hline produits & 19,3 & 16,6 & $\begin{array}{l}20,2 \\
28,5\end{array}$ & $\begin{array}{l}17,5 \\
170\end{array}$ & 20,8 & 21,1 & 19,1 & 17,5 & 20,2 & 29,0 & 21,2 & 22,2 & 15,1 & 13,6 & 15,8 & 17,8 & 21,1 & 26,5 & 22,1 & 22,8 & 30,0 & 28,0 \\
\hline & (33,0 & 31,0 & $\begin{array}{lll}28,5 & \end{array}$ & 17,99 & 22,6 & 19,6 & 22,5 & 23,4 & 19,5 & 11,9 & 19,9 & 21,1 & 23,8 & 24,5 & 26,2 & 22,2 & 29,1 & 23,4 & 27,1 & 29,6 & 27,4 & 0,5 \\
\hline $\begin{array}{l}\text { Nbre d'animaux } \\
\text { Bectes }\end{array}$ & 0,0 & 0,0 & 2,5 & 0,0 & 0,0 & 0,0 & 0,0 & 0,0 & 0,0 & 0,0 & 0,0 & 0,0 & 0,3 & 0,0 & 0,0 & 0,0 & 0,1 & $\begin{array}{l}0,0 \\
34\end{array}$ & 0,0 & 0,0 & 0,0 & $\begin{array}{l}0,0 \\
29\end{array}$ \\
\hline $\begin{array}{l}\text { Recettes } \\
\text { Revenu }\end{array}$ & 3.6 & 1,9 & 1,8 & 2,1 & 2,1 & 2,6 & 2,2 & 1,5 & 1,7 & 2,7 & 2.0 & 3,4 & 2.7 & $\begin{array}{l}0,0 \\
2.8\end{array}$ & $\begin{array}{l}0,0 \\
3,6\end{array}$ & $\begin{array}{l}0,0 \\
4,6\end{array}$ & $\begin{array}{l}4,3 \\
4,4\end{array}$ & $\begin{array}{l}3,4 \\
6,8\end{array}$ & $\begin{array}{l}0,1 \\
4,6\end{array}$ & $\begin{array}{l}2,6 \\
3,8\end{array}$ & $\begin{array}{l}3,2 \\
4,9\end{array}$ & $\begin{array}{l}2,9 \\
3,7\end{array}$ \\
\hline Avec exceptions & & & & & & & & & & & 17,6 & 20,6 & 18,0 & 19,5 & 19,9 & 17,0 & 17,4 & 16,1 & 22,3 & 24,3 & 21,8 & 18,7 \\
\hline Sans exceptions & 1,5 & 0,7 & 0,5 & & & & & & & & & & & & & & & 3,4 & 0,0 & 2,3 & 3,1 & 2,9 \\
\hline
\end{tabular}


Tableau annexe 22. OCDE : composition de l'ESP par catégorie et étiquette, 1986-2007 Part en pourcentage de l'ESP

\begin{tabular}{|c|c|c|c|c|c|c|c|c|c|c|c|c|c|c|c|c|c|c|c|c|c|c|}
\hline $\begin{array}{l}\text { PSE (millions USD) } \\
\text { PS }\end{array}$ & $\frac{1986}{230219}$ & $\begin{array}{l}1987 \\
248058\end{array}$ & $\frac{1988}{239531}$ & $\frac{1989}{224531}$ & $\begin{array}{l}1990 \\
247439\end{array}$ & $\frac{1991}{267935}$ & $\frac{1992}{262079}$ & $\frac{1993}{261567}$ & $\frac{1994}{271444}$ & $\frac{1995}{267489}$ & $\begin{array}{l}1996 \\
253938\end{array}$ & $\frac{1997}{232947}$ & $\frac{1998}{252502}$ & $\begin{array}{l}1999 \\
270788\end{array}$ & $\begin{array}{l}2000 \\
243722\end{array}$ & $\frac{2001}{218820}$ & 2002 & $\begin{array}{l}2003 \\
258203\end{array}$ & $\begin{array}{l}2004 \\
283037\end{array}$ & 2005 & $\begin{array}{l}2006 \\
257287\end{array}$ & $\frac{2007}{258236}$ \\
\hline \multirow{2}{*}{\multicolumn{23}{|c|}{ c labels correspondants }} \\
\hline $\begin{array}{l}\text { Production } \\
\text { Pat }\end{array}$ & & & & & & & & & & & & & & & & & & & & & & \\
\hline $\begin{array}{l}\text { Intrants } \\
S S A / B / B y\end{array}$ & 8,0 & 8,3 & 9,0 & 9,5 & 9,6 & 8,6 & 9,1 & 8,9 & 8,6 & 8,7 & 9,6 & 10,5 & 8,3 & 8,0 & 8,2 & 9,3 & 8,7 & 9,3 & 9,2 & 10,1 & 11,4 & 12,6 \\
\hline $\begin{array}{l}\text { Superficie } \\
\text { Sultion }\end{array}$ & 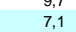 & $\begin{array}{l}9,0 \\
6,6\end{array}$ & $\begin{array}{c}8,0 \\
5,4\end{array}$ & 5,0 & $\begin{array}{l}8,4 \\
5,0\end{array}$ & $\begin{array}{l}4,9 \\
4,2\end{array}$ & $\begin{array}{r}0,6 \\
6,8\end{array}$ & $\begin{array}{r}9,3 \\
9,6\end{array}$ & $\begin{array}{l}14,9 \\
10,5\end{array}$ & $\begin{array}{l}18,0 \\
12,3\end{array}$ & $\begin{array}{l}20,3 \\
14,6\end{array}$ & $\begin{array}{l}20,1 \\
14,5\end{array}$ & $\begin{array}{l}20,4 \\
15,0\end{array}$ & $\begin{array}{l}1,2 \\
14,5 \\
1\end{array}$ & $\begin{array}{l}17,2 \\
17,2\end{array}$ & $\begin{array}{l}24,4 \\
19,2\end{array}$ & $\begin{array}{l}24,0 \\
17,9\end{array}$ & $\begin{array}{l}25,2 \\
17,6\end{array}$ & $\begin{array}{l}2,0 \\
19,2\end{array}$ & $\begin{array}{l}28,5 \\
23,2\end{array}$ & $\begin{array}{l}32,5 \\
28,0 \\
-1\end{array}$ & \\
\hline Nore d'animaux & 1,4 & 1,5 & 2,0 & 2,1 & 2,5 & 2,5 & 2,8 & 3,0 & 3,7 & 4,9 & 5,0 & 4,6 & 4,3 & 3,7 & 3,0 & 3,3 & 4,4 & 4,6 & 5,1 & 2,7 & 1,9 & 2,1 \\
\hline $\begin{array}{l}\text { Recettes } \\
\text { Revenu }\end{array}$ & 0,3 & 0,3 & 0,1 & 0,1 & 0,2 & 0,6 & 0,4 & 0,2 & 0,2 & 0,2 & 0,2 & 0,1 & 0,2 & 0,1 & 0,4 & 0,4 & 0,5 & 1,2 & 0,3 & 1,2 & 0,8 & 1,2 \\
\hline $\begin{array}{l}\text { Revenu } \\
\text { Autres produits }\end{array}$ & & & $\begin{array}{l}0,6 \\
0.8\end{array}$ & $\begin{array}{l}0,1 \\
1,0\end{array}$ & 0,6 & $\begin{array}{l}0,6 \\
1,9\end{array}$ & $\begin{array}{l}0,6 \\
1,4\end{array}$ & $\begin{array}{l}0,5 \\
1.3 \\
1.3\end{array}$ & & $\begin{array}{l}0,6 \\
1,9 \\
0\end{array}$ & $\begin{array}{l}0,6 \\
1,3 \\
1,3\end{array}$ & $\begin{array}{l}0,8 \\
1.0\end{array}$ & 0,9 & 0,8 & $\begin{array}{l}1,1 \\
0,8\end{array}$ & $\begin{array}{l}1,5 \\
1,3 \\
1,3\end{array}$ & 1,2 & $\begin{array}{l}1,7 \\
1,4\end{array}$ & 1,3 & & & $\begin{array}{l}1,7 \\
1,5\end{array}$ \\
\hline Courant & 98,7 & 99,1 & 99,0 & 99,0 & 99,3 & 99,4 & 99,1 & 99,1 & 98,6 & 98,7 & $\frac{1,0}{96,5}$ & 96,2 & 95,7 & $\begin{array}{l}94,6 \\
94,9\end{array}$ & 94,0 & $\frac{1,0}{94,0}$ & $\frac{1,5}{94,4}$ & 94,5 & $\frac{1,1}{93,5}$ & 85,5 & $\frac{1,9}{78,8}$ & $\frac{1,5}{75,9}$ \\
\hline Non-courant & 1,3 & 0,9 & 1,0 & 1,0 & 0,7 & 0,6 & 0,9 & 0,9 & 1,4 & 1,3 & 3,5 & 3,8 & 4,3 & 5,1 & 6,0 & 6,0 & 5,6 & 5,5 & 6,5 & 14,5 & 21,2 & \\
\hline $\begin{array}{l}\text { Courantt/variable } \\
\text { Couranttifee }\end{array}$ & $\begin{array}{l}86,8 \\
115\end{array}$ & $\begin{array}{l}86,3 \\
123\end{array}$ & $\begin{array}{l}83,9 \\
14,3\end{array}$ & $\begin{array}{l}82,9 \\
150\end{array}$ & 82,1 & $\begin{array}{l}83,6 \\
13,9\end{array}$ & $\begin{array}{l}81,2 \\
165\end{array}$ & $\begin{array}{l}78,6 \\
191\end{array}$ & $\begin{array}{l}78,0 \\
19,5\end{array}$ & 72,4 & 68,8 & 68,2 & 70,5 & 72,3 & 68,9 & 64,8 & 66,2 & 64,1 & 62,8 & 58,2 & 54,2 & 51,1 \\
\hline ariable & 0,1 & $\begin{array}{r}0,1 \\
0,1\end{array}$ & $\begin{array}{r}14,0 \\
0,1\end{array}$ & $\begin{array}{r}1,0 \\
0,1\end{array}$ & $\begin{array}{l}1,0,0 \\
0,0\end{array}$ & $\begin{array}{r}3,9 \\
0,0\end{array}$ & $\begin{array}{r}0,5 \\
0,0\end{array}$ & 0,0 & $\begin{array}{r}19,0 \\
0,0\end{array}$ & $\begin{array}{r}24,4 \\
0,4\end{array}$ & $\begin{array}{c}26,4 \\
0,3\end{array}$ & $\begin{array}{r}27,0 \\
0,0\end{array}$ & $\begin{array}{r}24,3 \\
1,1\end{array}$ & $\begin{array}{r}21,8 \\
20\end{array}$ & $\begin{array}{r}24,3 \\
23\end{array}$ & $\begin{array}{r}27,9 \\
2,3\end{array}$ & $\begin{array}{r}26,8 \\
10\end{array}$ & $\begin{array}{r}29,0 \\
0.4\end{array}$ & $\begin{array}{r}29,6 \\
16\end{array}$ & $\begin{array}{r}25,9 \\
19\end{array}$ & $\begin{array}{l}22,1 \\
0.9\end{array}$ & \\
\hline Non-courant fixe & 1,3 & 0,9 & 0,9 & 1,0 & 0,7 & 0,6 & 0,9 & 0,9 & 1,4 & 0,9 & 3,2 & 3,8 & 3,1 & 3,1 & 3,7 & 3,8 & 4,6 & 5,2 & $\begin{array}{l}1,0 \\
4,9\end{array}$ & 12,6 & 20,3 & 23,4 \\
\hline $\begin{array}{l}\text { Autres produits } \\
\text { Production requise }\end{array}$ & 0,3 & $\begin{array}{r}0,4 \\
98,8\end{array}$ & $\begin{array}{r}0,8 \\
98,3\end{array}$ & $\begin{array}{r}1,0 \\
98,0\end{array}$ & $\begin{array}{r}0,9 \\
98,5 \\
\end{array}$ & $\begin{array}{r}1,9 \\
975\end{array}$ & 1,4 & 1,3 & 1,1 & $\begin{array}{r}1,9 \\
970\end{array}$ & $\frac{1,3}{954}$ & $\begin{array}{r}1,0 \\
953\end{array}$ & 0,9 & 0,8 & $\begin{array}{r}0,8 \\
932\end{array}$ & $\begin{array}{r}1,3 \\
927\end{array}$ & $\frac{1,5}{930}-1$ & 1,4 & & 1,4 & $\frac{1,9}{772}$ & $\frac{1,5}{750}$ \\
\hline $\begin{array}{l}\text { rouduction requise } \\
\text { Un seul produit }\end{array}$ & $\begin{array}{l}98,8 \\
98,8\end{array}$ & $\begin{array}{l}98,8 \\
9888\end{array}$ & 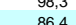 & $\begin{array}{l}98,0 \\
950\end{array}$ & $\begin{array}{l}98,5 \\
889 \\
8\end{array}$ & 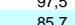 & $\begin{array}{l}9,1 \\
98,1\end{array}$ & $\begin{array}{l}9,9 \\
815\end{array}$ & $\begin{array}{ll}97,6 \\
886\end{array}$ & $\begin{array}{l}97,0 \\
764\end{array}$ & 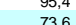 & 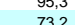 & 94,9 & 94,1 & 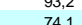 & 92,7 & 93,0 & 93,3 & 92,6 & 84,4 & 77,3 & $\begin{array}{l}75,0 \\
54,3\end{array}$ \\
\hline $\begin{array}{l}\text { Un seul profou } \\
\text { Un groupe de }\end{array}$ & ${ }_{35}^{0,2}$ & 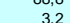 & $\begin{array}{lll}0.46 \\
46\end{array}$ & 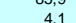 & 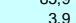 & 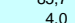 & 年, & 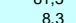 & $\begin{array}{l}0.0 \\
9,0,0\end{array}$ & 114 & $\begin{array}{l}\underbrace{}_{120} \\
120\end{array}$ & (119 & 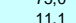 & 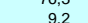 & 89 & 9,1 & $\begin{array}{ll}2,0 \\
95\end{array}$ & $\begin{array}{l}x_{10,6} \\
105\end{array}$ & 作, & 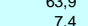 & $\begin{array}{c}5,6 \\
50 \\
50\end{array}$ & $\begin{array}{r}54,3 \\
5,2\end{array}$ \\
\hline Tous les produ & 7,1 & 6,9 & 7,4 & 8.0 & 8.6 & 7,8 & 8,4 & 8.0 & 8,0 & 9,2 & $\begin{array}{l}9,8 \\
9,8\end{array}$ & 10,2 & 8.8 & 8,6 & 10,2 & 12,1 & $\begin{array}{lll}10,7 & \end{array}$ & 12,2 & 12,0 & 13,1 & 14,8 & $\begin{array}{r}0,2 \\
15,5\end{array}$ \\
\hline \multicolumn{23}{|l|}{$\frac{\text { Production facult }}{\text { Proportion de cha }}$} \\
\hline $\begin{array}{l}\text { Proportion de cha } \\
\text { Avec linites }\end{array}$ & 28,6 & 30,6 & 24,2 & & 26,9 & 25,3 & 29,1 & 30,8 & 29,8 & 29,5 & 34,3 & 36,7 & 40,4 & & 37,8 & 37,8 & 37,3 & 38,4 & 40,6 & 40,2 & & \\
\hline Sans limites & 71,2 & 69,3 & 75. & $\begin{array}{c}72,9 \\
159\end{array}$ & 73,1 & 74 & $\begin{array}{r}70,5 \\
174\end{array}$ & 69,1 & 70,3 & 69,8 & $\begin{array}{c}65,6 \\
295\end{array}$ & 63,6 & 59,8 & 61, & & & 62. & & & 59,3 & & 59,1 \\
\hline & $\begin{array}{l}12,8 \\
86,9\end{array}$ & $\begin{array}{l}13,2 \\
86,4\end{array}$ & $\begin{array}{l}15,2 \\
84,0\end{array}$ & 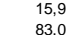 & $\begin{array}{l}17,0 \\
82.1\end{array}$ & $\begin{array}{l}14,5 \\
83,6\end{array}$ & $\begin{array}{l}17,4 \\
81.2\end{array}$ & $\begin{array}{l}20,0 \\
786\end{array}$ & $\begin{array}{l}20,9 \\
780\end{array}$ & $\begin{array}{l}25,3 \\
728\end{array}$ & 29,5 & $\begin{array}{l}30,8 \\
68,2\end{array}$ & $\begin{array}{l}27,4 \\
717\end{array}$ & $\begin{array}{l}24,9 \\
74,3\end{array}$ & & & $\begin{array}{l}31,4 \\
67,1\end{array}$ & $\begin{array}{l}34,2 \\
64,5\end{array}$ & & $\begin{array}{l}38,5 \\
60,1\end{array}$ & 3,0 & \\
\hline Avec c. (obligatoire) & 3,7 & 2,7 & 3,6 & $\begin{array}{r}80,5 \\
2,5\end{array}$ & $\begin{array}{l}0<, 1 \\
1,7\end{array}$ & $\begin{aligned} 0,0 \\
1,4\end{aligned}$ & $\begin{array}{l}0,2 \\
1,7\end{array}$ & $\begin{array}{r}3,0 \\
3,3\end{array}$ & $\begin{array}{l}1,0,6 \\
2,6\end{array}$ & 2,4 & $\begin{array}{l}0,1,4 \\
4,4\end{array}$ & $\begin{array}{l}0,6 \\
4,9 \\
4,9\end{array}$ & 7,8 & 10,8 & $\begin{array}{l}12,0 \\
12,0\end{array}$ & $\begin{array}{l}12,2 \\
12,0\end{array}$ & $\begin{array}{r}6,1 \\
8,2\end{array}$ & $\begin{array}{r}64,5 \\
7,2\end{array}$ & $\begin{array}{l}64,4 \\
16,6\end{array}$ & $\begin{array}{l}60,1 \\
20,1\end{array}$ & $\begin{array}{l}5,1 \\
2,2\end{array}$ & $\begin{array}{l}3,0 \\
23,2\end{array}$ \\
\hline & $\begin{array}{r}0,6 \\
189\end{array}$ & $\begin{array}{c}0,9 \\
188\end{array}$ & ${ }_{18,2}^{1,2}$ & 1,8 & 2,0 & $\begin{array}{r}2,2 \\
\end{array}$ & & 3,7 & & 5 & $\begin{array}{r}6,5 \\
231\end{array}$ & $\begin{array}{r}6,8 \\
03\end{array}$ & 6,6 & & 5,8 & 6,8 & & 7,3 & 7,9 & 7,4 & 7,9 & 7,9 \\
\hline & $\begin{array}{l}18,9 \\
882\end{array}$ & $\begin{array}{l}18,8 \\
888\end{array}$ & $\begin{array}{l}18,1 \\
864\end{array}$ & $\begin{array}{l}19,6 \\
85,9\end{array}$ & $\begin{array}{l}20,9 \\
85,9\end{array}$ & $\begin{array}{l}18,0 \\
85,7\end{array}$ & $\begin{array}{l}19,3 \\
83,9\end{array}$ & $\begin{array}{l}19,1 \\
81,5\end{array}$ & & & $\begin{array}{l}23,1 \\
736\end{array}$ & & & $\begin{array}{l}18,7 \\
763\end{array}$ & $\begin{array}{l}20,7 \\
741\end{array}$ & $\begin{array}{l}23,9 \\
707\end{array}$ & $\begin{array}{l}23,3 \\
728\end{array}$ & & & $\begin{array}{l}18,6 \\
639\end{array}$ &, 6 & \\
\hline & 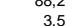 & $\begin{array}{r}8,8 \\
3,2\end{array}$ & $\begin{array}{r}80,4 \\
4,6\end{array}$ & $\begin{array}{l}85,1 \\
4,1\end{array}$ & $\begin{array}{r}85,9 \\
3,9\end{array}$ & $\begin{array}{r}85,7 \\
4,0\end{array}$ & $\begin{array}{r}83,9 \\
5,5\end{array}$ & $\begin{array}{r}8,5 \\
8,3\end{array}$ & $\begin{array}{r}80,6 \\
9,0\end{array}$ & $\begin{array}{l}76,4 \\
11,4\end{array}$ & $\begin{array}{l}7,6 \\
12,0\end{array}$ & $\begin{array}{l}7,3,2 \\
11,9\end{array}$ & 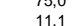 & 9,2 & $\begin{array}{r}4,1 \\
8,9\end{array}$ & $\begin{array}{r}9,7 \\
9,9\end{array}$ & $\begin{array}{r}2,8 \\
9,5\end{array}$ & $\begin{array}{l}\begin{array}{l}10,6 \\
10,5\end{array} \\
\text { a }\end{array}$ & $\begin{array}{l}10,0 \\
10,5\end{array}$ & $\begin{array}{r}7,9 \\
7,4\end{array}$ &, 0 & $\begin{array}{l}5,3 \\
5,2 \\
5\end{array}$ \\
\hline produits & 7,1 & 6,9 & 7,4 & 8,0 & 8,6 & 7,8 & 8,4 & 8,0 & 8,0 & 9,2 & 9,8 & 10,2 & 8,8 & 8,6 & 10,2 & 12,1 & 10,7 & 12,2 & 12,0 & 13,1 & 14,8 & 15,5 \\
\hline & 7,1 & 6,6 & 5,4 & 5,0 & 5,0 & 4,2 & 6,8 & 9,6 & 10,5 & 12,3 & 14,6 & 14,5 & 15,0 & 14,5 & & 19,2 & 17,9 & 17,6 & 19,2 & 23,2 & 28,0 & 29,8 \\
\hline & 1,4 & 1,5 & 2,0 & 2,1 & $\begin{array}{l}2,5 \\
0,2\end{array}$ & 2,5 & 2,8 & 3,0 & 3,7 & 4,9 & 5,0 & 4,6 & 4,3 & 3,7 & 3,0 & 3,3 & 4,4 & 4,6 & 5, & 2,7 & 1,9 & 2,1 \\
\hline & 0,9 & 0,6 & 0.6 & 0.7 & 0,6 & 0.6 & 0,6 & 0,5 & 0,5 & $\begin{array}{l}0,2 \\
0,6\end{array}$ & $\begin{array}{l}0,2 \\
0,6\end{array}$ & $\begin{array}{l}0,1 \\
0,8\end{array}$ & $\begin{array}{l}0,2 \\
0,9\end{array}$ & 0.8 & $\begin{array}{l}0,4 \\
1,1\end{array}$ & $\begin{array}{l}0,4 \\
1,5\end{array}$ & $\begin{array}{l}0,5 \\
1,2\end{array}$ & $\begin{array}{l}1,2 \\
1,7\end{array}$ & $\begin{array}{l}0,3 \\
1,3\end{array}$ & 1,4 & $\begin{array}{l}0,8 \\
1,9\end{array}$ & \\
\hline xceptions & 0,6 & 0,6 & 0,7 & 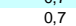 & 0,5 & 0,5 & 0.4 & 0,3 & 0,8 & 0,7 & 2,9 & & & & 5,3 & 5,1 & 4,2 & 3.4 & 4 & t. & 88 & \\
\hline is exceptions & 0,3 & 0.2 & 0,2 & 0.2 & 0,2 & 0.2 & 0.5 & 0.4 & 0.5 & 0.4 & $\begin{array}{l}0.4 \\
0.4\end{array}$ & 0,1 & 0,0 & 0.3 & 0.7 & 0.9 & 1.4 & 1,9 & 2,0 & 6.0 & 7.0 & \\
\hline
\end{tabular}

